\title{
Struktur, Wachstum und Phasenumwandlungen dünner Eisen-Palladium Schichten
}

\author{
Dissertation \\ zur Erlangung des mathematisch-naturwissenschaftlichen Doktorgrades \\ "Doctor rerum naturalium" \\ der Georg-August-Universität zu Göttingen
}

vorgelegt von

Tobias Edler

aus Steinheim am Albuch

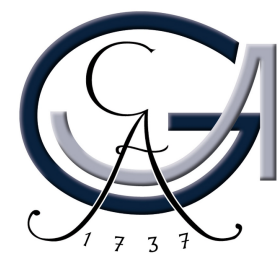


Referent

Korreferent
Prof. Dr. Stefan G. Mayr Prof. Dr. Reiner Kirchheim

Tag der mündlichen Prüfung:

15.6.2010 


\section{Inhaltsverzeichnis}

1 Einleitung 1

2 Grundlagen $\quad 5$

2.1 Das System Eisen-Palladium . . . . . . . . . . . . 5

2.1.1 Martensitische Umwandlungen . . . . . . . . . . 7

2.1.2 Der Invar-Effekt . . . . . . . . . . . . . . . . 13

2.1.3 Instabilität des Kristallgitters . . . . . . . . . . . 14

2.1.4 Magnetostriktion ... . . . . . . . . . . . 14

2.1.5 Thermischer und magnetischer Formgedächtniseffekt . 15

2.2 Wachstum dünner Filme . . . . . . . . . . . . . . . . . 17

2.3 Ionenbestrahlung von Festkörpern . . . . . . . . . . . . . 21

3 Experimentelle Methoden $\quad 27$

3.1 Herstellung dünner Schichten . . . . . . . . . . . . 27

3.1.1 Elektronenstrahlverdampfen . . . . . . . . . . . 27

3.1.2 Gepulste Laserdeposition . . . . . . . . . . . . . . . 30

3.1.3 Aufdampfen mit Ionenstrahlunterstützung . . . . . . . 31

3.2 Aufbau zur Messung mechanischer Spannungen . . . . . . . . 32

3.3 Tempern der Proben . . . . . . . . . . . . . . . . 36

3.4 Präparation freistehender Filme . . . . . . . . . . 36

3.5 Röntgenstreumethoden . . . . . . . . . . . . . . . . 39

3.6 Elektronenmikroskopie . . . . . . . . . . . . . . . . . 44

3.7 Rastersondenverfahren . . . . . . . . . . . . . . . . 45

3.8 Rutherford-Rückstreuung . . . . . . . . . . . . . . . . 46

3.9 Magnetisierungsmessungen . . . . . . . . . . . . . . . 48

4 Molekulardynamiksimulationen $\quad 51$

4.1 Verwendetes Potential . . . . . . . . . . . . . . 51

4.2 Ablauf der Simulationen . . . . . . . . . . . . . . 52

4.3 Der Zentralsymmetrie-Parameter ........... . . 53 
$\begin{array}{llr}5 & \text { Ergebnisse } & 57\end{array}$

5.1 Polykristalline Schichten . . . . . . . . . . . . . . 57

5.1.1 Wachstum auf Siliziumdioxid . . . . . . . . . . 58

5.1.2 Aufdampfen mit Ionenstrahl-Unterstützung . . . . . . 62

5.1.3 Nachträgliches Tempern der Filme . . . . . . . . . . 63

5.1.4 Mit hochenergetischen Ionen bestrahlte Filme . . . . . 64

5.2 Epitaktische Schichten . . . . . . . . . . . . . 66

5.2.1 Mittels PLD hergestellte Schichten . . . . . . . . 67

5.2.2 Aufgedampfte Schichten . . . . . . . . . . . . 72

5.3 Molekulardynamik-Simulationen . . . . . . . . . . . . . 77

5.4 Freistehende Schichten . . . . . . . . . . . . . . . . . 84

5.5 Martensitische Umwandlungen . . . . . . . . . . . . . . . . . . 90

5.5.1 Polykristalline Filme auf $\mathrm{SiO}_{2}$. . . . . . . . . . . . 90

5.5.2 Freistehende einkristalline Filme . . . . . . . . . . . 95

6 Diskussion 101

6.1 Kristallstruktur während des Wachstums . . . . . . . . . . . . 101

6.1.1 Bei hohen Temperaturen auf $\mathrm{SiO}_{2}$ aufgedampfte Filme 102

6.1.2 Ionenstrahlunterstützte Deposition . . . . . . . . . . 103

6.1.3 Gepulste Laserdeposition . . . . . . . . . . . . . . 106

6.1.4 Epitaktisches Aufdampfen . . . . . . . . . . . 107

6.2 Mechanische Spannungen . . . . . . . . . . . . . . . . . 107

6.2.1 Bei Raumtemperatur laserdeponierte Schichten . . . . 108

6.2.2 Bei hohen Temperaturen aufgedampfte Schichten . . . 111

6.3 Veränderung der Kristallstruktur . . . . . . . . . . . . . . 115

6.3.1 Hochenergetische Ionenbestrahlung . . . . . . . . . 115

6.3.2 Nachträgliche Temperaturbehandlung . . . . . . . . . . 116

6.4 Elastische Eigenschaften . . . . . . . . . . . . . . . . . . . . 118

6.4.1 Polykristalline Schichten . . . . . . . . . . . . 118

6.4.2 Abgelöste freistehende Schichten . . . . . . . . . . . . . 119

6.5 Strukturelle Übergänge . . . . . . . . . . . . . . . . . . . . . . 121

6.5.1 Polykristalline Schichten . . . . . . . . . . . . . 121

6.5.2 Reversibler fcc-bcc-Übergang in getemperten abgelösten

Proben . . . . . . . . . . . . . . . . 123

$\begin{array}{lll}7 & \text { Zusammenfassung und Ausblick } & 127\end{array}$

A Spannungsmessungen bei schrägem Einfall 131

$\begin{array}{ll}\text { Literaturverzeichnis } & 134\end{array}$ 


\section{Kapitel 1}

\section{Einleitung}

Eisen-Palladium ist ein modernes Funktionsmaterial, das eine große Bandbreite an interessanten physikalischen Effekten zeigt, die in vielfältigen technischen Anwendungen genutzt werden können. Aufgrund seiner magnetischen Anisotropie senkrecht zur Filmebene ist es für die magnetische Datenspeicherung mit hoher Speicherdichte sehr interessant [1, 2]. Zusätzlich zeigt es den Invar-Effekt, hat also in einem gewissen Temperaturbereich eine sehr geringe thermische Ausdehnung [3, 4, 5] und durchläuft einen Martensitübergang [6]. Dies ermöglicht unter anderem den thermischen und magnetischen Formgedächtnis-Effekt [7, 8, 9, 10], der sich zum Beispiel in Aktoren nutzen lässt. Der inverse magnetische Formgedächtnis-Effekt, also eine durch Dehnung induzierte Änderung der Magnetisierung, kann darüber hinaus die Grundlage für hochempfindliche Dehnungsmessungen bilden [11].

Quantenmechanische Rechnungen sagen voraus, dass sich $\mathrm{Fe}_{70} \mathrm{Pd}_{30}$ mit extrem geringem Energieaufwand dehnen lässt, und so entlang des BainPfads [12] von der fcc in eine bcc-Struktur überführt werden kann [13, 14, 15]. Diese Simulationen zeigen außerdem, dass die Randbedingungen und die Möglichkeiten, lokale Spannungen abzubauen, einen großen Einfluss auf die Stabilität der Phasen haben. Dies führt dazu, dass in verschiedenen Simulationen je nach Relaxation der Simulationszelle der Grundzustand unterschiedliche Gitterstrukturen aufweist.

Massivproben aus $\mathrm{Fe}_{70} \mathrm{Pd}_{30}$ wurden seit den 80er Jahren des 20. Jahrhunderts von vielen Gruppen weltweit, insbesondere in Japan, intensiv untersucht $[16,17,18,19,20,21,22,23]$. Der magnetische Formgedächtniseffekt (MSM-Effekt), der zuerst 1996 von Ullakko in NiMnGa gefunden wurde [24], konnte erst 1998 von James und Wuttig in Bulkproben bei der Zusammensetzung $\mathrm{Fe}_{70} \mathrm{Pd}_{30}$ nachgewiesen werden [7].

Dieser Effekt ist durch sein hohes Anwendungspotential für die Mikround Nanotechnologie von besonderem Interesse. Gerade dort ist es vorteil- 
haft, funktionale dünne Schichten zu verwenden, statt kleine Strukturen aus Bulkmaterial zu fertigen. Die Verwendung freistehender Schichten kommt einem weiteren Vorteils von MSM-Aktoren gegenüber konventionellen elektromagnetischen Aktoren entgegen, nämlich dass sie sich als planare Strukturen konzipieren lassen [25]. Obwohl auch in polykristallinen Materialien unter Umständen ein großer MSM-Effekt beobachtet werden kann [26], ist die Entwicklung von einkristallinen Filmen mit geeigneten Eigenschaften erstrebenswert, weil dort der maximale Effekt zu erwarten ist.

Dünne Schichten sind allerdings im Gegensatz zu Massivproben sehr viel mehr von den Eigenschaften der Ober- und Grenzflächen geprägt, so dass sich nicht alle Erkenntnisse, die an Bulkmaterial gewonnen wurden, direkt auf dünne Schichten übertragen lassen. Gerade mechanische Spannungen, die oftmals während der Schichtdeposition entstehen, können hier einen großen Einfluss haben. In Bulkproben ist bekannt, dass schon moderate Spannungen von $10 \mathrm{MPa}$ einen deutlichen Einfluss auf die Phasenstabilität haben [27]. So sind für dünne verspannte Schichten ebenfalls deutliche Effekte zu erwarten, in denen insbesondere der Einfluss des sehr viel dickeren Substrats eine wichtige Rolle spielen wird. Die Gründe für solch eine Verspannung dünner Filme können vielfältig sein, und reichen von den unterschiedlichen Gitterkonstanten und thermischen Ausdehnungen von Film und Substrat [28] bis hin zu Spannungen, die durch die Änderung der Oberflächenmorphologie beim Zusammenwachsen von einzelnen Inseln entstehen [29].

Daher soll in dieser Arbeit das Wachstum dünner polykristalliner und epitaktischer Eisen-Palladium Filme untersucht werden. Insbesondere ist hierbei der Einfluss der Wachstumsbedingungen und der auftretenden mechanischen Verspannungen von Interesse, um so die mikroskopischen Ursachen für die Eigenschaften der Filme zu verstehen und diese gezielt zu beeinflussen.

Zur Untersuchung der intrinsischen Eigenschaften dünner Filme, ganz besonders von Filmen, die heteroepitaktisch auf einem einkristallinen Substrat hergestellt wurden, ist es notwendig, diese Filme vom Substrat zu lösen. Deshalb wurde im Rahmen dieser Arbeit ein Verfahren entwickelt, um FePd-Filme ohne Opferschicht direkt von MgO-Substraten abzulösen. Dies ermöglicht es, den Einfluss des Substrats und der durch die Wechselwirkung mit dem Substrat in den Filmen gebildeten Defekte auf die Stabilität verschiedener Gitterstrukturen zu untersuchen. Somit können die theoretischen Vorhersagen nicht nur mit Filmen auf einem harten Substrat, sondern auch mit dünnen, freistehenden Einkristallen verglichen werden.

Im nun folgenden Kapitel 2 soll der Stand der Forschung auf den für diese Arbeit relevanten Gebieten zusammengefasst werden. Daran schließt sich 
ein Kapitel über die experimentellen Methoden an (Kapitel 3), in dem die für die Interpretation der Messdaten notwendigen Eigenschaften dieser Methoden dargestellt sind. Den Grundlagen der Molekulardynamiksimulationen ist das darauffolgende Kapitel 4 gewidmet. Die gewonnenen Ergebnisse aus den Experimenten und Simulationen werden daraufhin in Kapitel 5 gemeinsam dargestellt und in Kapitel 6 diskutiert und mit anderen Ergebnissen verglichen. Das abschließende Kapitel 7 fasst die wichtigsten Ergebnisse der vorliegenden Arbeit noch einmal zusammen, und bietet einen Ausblick auf mögliche zukünftige Fragestellungen. 


\section{Kapitel 2}

\section{Grundlagen}

Dieses Kapitel fasst in kurzer Form den Stand der Forschung auf den für diese Arbeit relevanten Bereichen zusammen und führt die wichtigsten Begrifflichkeiten ein. Nach einigen allgemeinen Bemerkungen zum Materialsystem Eisen-Palladium werden am Beispiel dieses Systems der Invar-Effekt, die Grundlagen einer martensitischen Umwandlung und der damit zusammenhängende thermische und magnetische Formgedächtniseffekt dargestellt. Daran schließen sich Ausführungen zum Wachstum dünner Filme und den wichtigsten Effekten bei der Wechselwirkung zwischen Ionen und Festkörpern an.

\subsection{Das System Eisen-Palladium}

Zum Verständnis der physikalischen Vorgänge in einem Legierungssystem ist zunächst eine Analyse des Phasendiagramms dieses Systems notwendig, aus dem sich bei einer gegebenen Temperatur und Zusammensetzung die im thermodynamischen Gleichgewicht zu erwartenden Phasen ablesen lassen. Im Fall von Eisen-Palladium (dargestellt in Abbildung 2.1) erkennt man, dass sich unterhalb der Schmelze (mit L für Liquidus bezeichnet) zunächst ein Gebiet anschließt, in dem die $\gamma$-Phase für alle Zusammensetzungen stabil ist. Hierbei handelt es sich um eine kubisch-flächenzentrierte (fcc) Struktur, in der Eisen- und Palladiumatome regellos auf den Plätzen des Gitters verteilt sind. Dieses Gebiet erstreckt sich über den Temperaturbereich zwischen dem Schmelzpunkt (je nach Zusammensetzung zwischen $1310^{\circ} \mathrm{C}$ und $1550{ }^{\circ} \mathrm{C}$ ) bis zu Temperaturen deutlich unterhalb von $900^{\circ} \mathrm{C}$.

In dem für diese Arbeit relevanten Konzentrationsbereich um $\mathrm{Fe}_{70} \mathrm{Pd}_{30}$ liegt die untere Grenze dieser fcc-Phase bei Temperaturen um $760{ }^{\circ} \mathrm{C}$. Bei einem infinitesimal langsamen Abkühlprozess, der als Folge von thermischen 


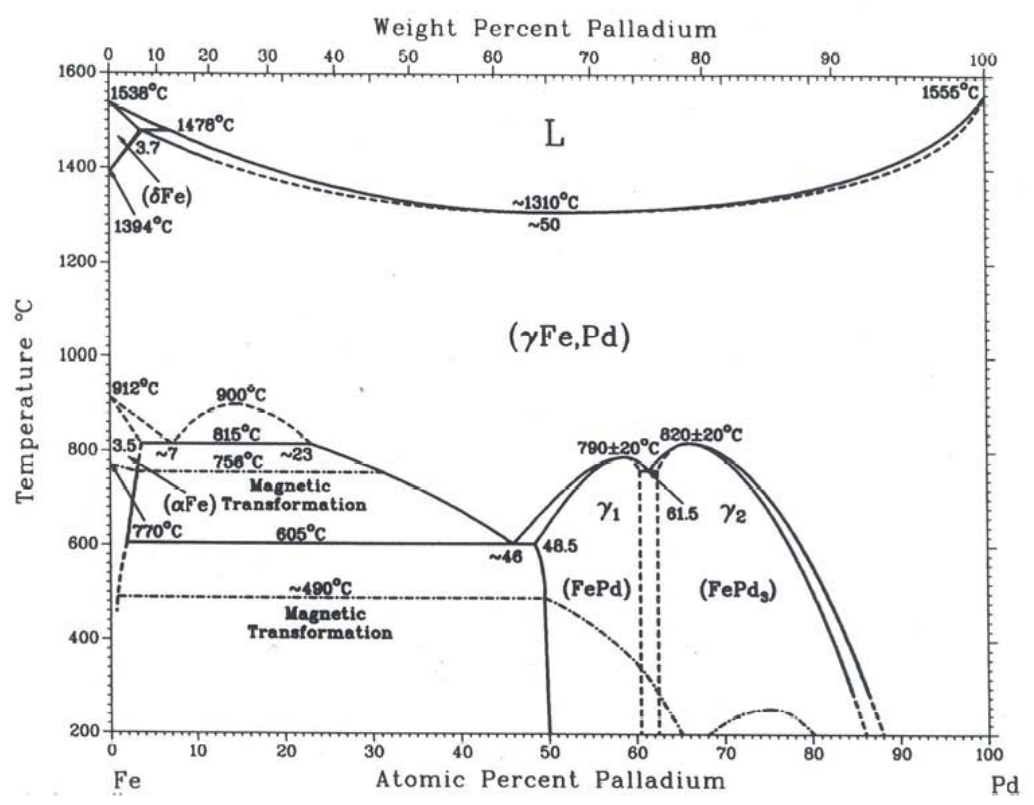

Abbildung 2.1: Phasendiagramm für Eisen-Palladium, aus [30].

Gleichgewichtszuständen beschrieben werden kann, wäre beim Erreichen dieser Temperatur ein Entmischen in eine $\alpha$-Eisen-Phase mit einem geringen Palladiumgehalt von unter $3,5 \%$ und eine an Eisen verarmte $\gamma$-Phase zu erwarten, deren jeweilige Anteile sich nach dem Hebelgesetz [31] bestimmen lassen. Beim weiteren Abkühlen entlang der unteren Grenzlinie der $\gamma$-Phase würde deren Palladiumkonzentration immer weiter bis ungefähr $46 \%$ bei $600{ }^{\circ} \mathrm{C}$ steigen, bei noch tieferen Temperaturen würde das System in eine Eisenphase mit einem Palladiumgehalt von unter einem Prozent (der durch die Mischungsentropie stabilisierten Randlöslichkeit) und eine $\mathrm{Fe}_{50} \mathrm{Pd}_{50}$-Phase entmischen.

Diese $\mathrm{Fe}_{50} \mathrm{Pd}_{50}$-Phase weist eine $\mathrm{L1}_{0}$-Struktur auf, bei der es sich um eine geordnete, leicht tetragonal verzerrte flächenzentrierte Struktur handelt: In ihr wechseln sich unverzerrte Ebenen aus Eisen- und Palladiumatomen ab, deren Ebenenabstand nicht mit dem Atomabstand innerhalb dieser Ebenen übereinstimmt, so dass sie ein entlang einer Achse verzerrtes fcc-ähnliches Gitter bilden, das auch als fct-Gitter bezeichnet wird.

Die Bildung dieser Phasen kann unter Umständen sehr lange dauern, da im Festkörper eine Änderung des Eisengehalts einer Phase nur über die Diffusion von Eisenatomen aus der Phase hinaus geschehen kann. So ist es 
möglich, auch Nichtgleichgewichts-Strukturen zu erzeugen, indem man eine Probe aus einem Gebiet heraus, in dem die Struktur stabil ist, sehr schnell zu tiefen Temperaturen hin abschreckt. Bei hinreichend tiefen Temperaturen besteht zwar eine große treibende Kraft zur Strukturänderung, gleichzeitig ist aber die Kinetik des Systems so weit verlangsamt, dass diese Struktur in endlicher Zeit nicht erreicht wird, daher spricht man hierbei von Metastabilität. Ein sehr direktes Beispiel stellt das Ultra Rapid Quenching dar, bei dem ein geschmolzener Tropfen zwischen zwei Kupferstempeln abgeschreckt wird, wobei Abkühlraten von bis $\mathrm{zu} 10^{6} \mathrm{~K} / \mathrm{s}$ erreicht werden. Hiermit lassen sich direkt polykristalline $\mathrm{Fe}_{70} \mathrm{Pd}_{30}$-Proben herstellen [32], aber auch die Kondensation dünner Schichten aus der Dampfphase kann einen solchen Nichtgleichgewichts-Prozess darstellen, durch den metastabile Phasen erzeugt werden können [33, 34, 35].

\subsubsection{Martensitische Umwandlungen}

Beim Abkühlen von $\mathrm{Fe}_{70} \mathrm{Pd}_{30}$-Legierungen können in der metastabilen fccPhase verschiedene diffusionslose strukturelle Übergänge beobachtet werden. Ähnliche Übergänge werden auch in anderen Materialien beobachtet und wurden zuerst anhand des prominentesten Vertreters, dem System EisenKohlenstoff (Stahl) charakterisiert, und die dort gebildeten Begriffe später verallgemeinert. So bezeichnet man die Ausgangsphase hoher Symmetrie als Austenit $^{1}$, die bei der Umwandlung entstehende Phase niedriger Symmetrie als Martensit $^{2}$. Allgemein bezeichnet man eine diffusionslose Änderung der Struktur eines kristallinen Materials als martensitische Umwandlung, wenn sie folgenden Kriterien genügt [31]:

- Neben einer Volumenänderung tritt auch eine Gestaltänderung des transformierten Bereichs auf.

- Ein Martensit-Bereich besitzt eine Mikrostruktur, die von Gleitung oder Zwillingsbildung verursacht wird.

- Die Deformation bei der Umwandlung entspricht insgesamt bis auf eine Rotation im Wesentlichen einer Scherung parallel zu einer unverzerrt bleibenden Ebene, der so genannten Habitusebene.

- Zwischen den Strukturen vor und nach der Umwandlung besteht eine exakte Orientierungsbeziehung, bei der eine dichtest gepackte Ebene

\footnotetext{
${ }^{1}$ Nach dem britischen Metallurgen Sir William Chandler Roberts-Austen, $\star 1843+1902$

${ }^{2}$ Nach dem deutschen Metallurgen Adolf Martens $\star 1850+1914$
} 
des Austenits parallel zu einer dichtest gepackten Ebene des Martensits liegt.

- Im Gegensatz zu einer diffusionsgetriebenen Umwandlung legen die einzelnen Atome relativ zueinander nur Strecken zurück, die geringer als der atomare Abstand sind, und ändern ihre Abstände zueinander nur unwesentlich.

Da im Allgemeinen die Form eines transformierenden Bereichs durch die ihn umgebende Matrix fest vorgegeben ist, stellt sich entweder durch Gleitung oder durch Verzwillingung eine Mikrostruktur wie in Abbildung 2.2 ein, die die äußere Form des Bereichs näherungsweise wiederherstellt.

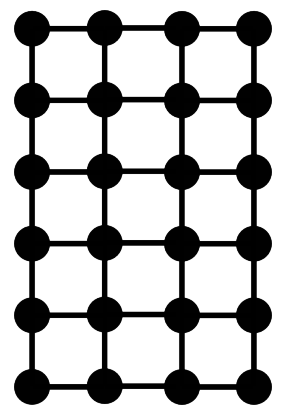

(a)

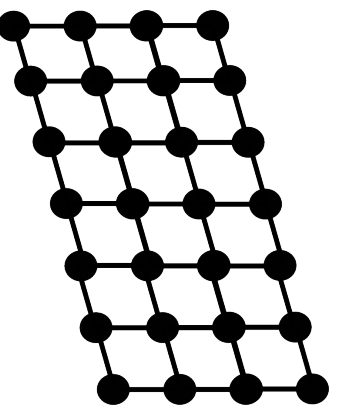

(b)

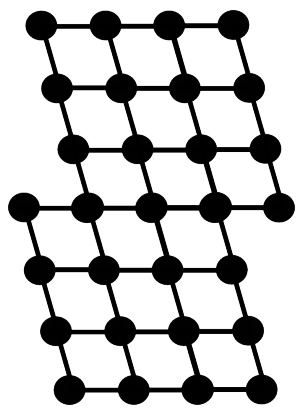

(c)

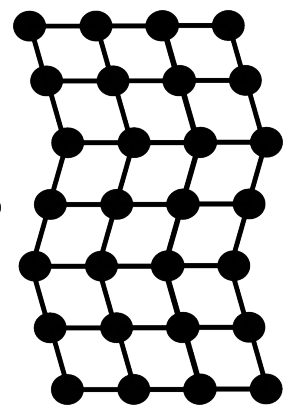

(d)

Abbildung 2.2: Schema einer martensitischen Umwandung von (a) nach (b). Die äußere Form des Kristallbereichs kann entweder durch Gleitung von vollen Versetzungen (c) oder durch Verzwillingung (d) näherungsweise wiederhergestellt werden.

Der für Stahl entwickelte Bain-Pfad-Formalismus [12] erlaubt es, die Beziehungen zwischen der martensitischen und der austenitischen Struktur auf elegante Art zu beschreiben: Man kann ein kubisch flächenzentriertes Gitter als stark verzerrtes raumzentriertes Gitter beschreiben, in dem man die in Abbildung 2.3 blau markierten Atome als Einheitszelle verwendet. Nun kann man die Gitterverzerrung als Matrix schreiben, die nur Einträge auf der Diagonalen hat:

$$
B=\left(\begin{array}{ccc}
\eta_{1} & 0 & 0 \\
0 & \eta_{2} & 0 \\
0 & 0 & \eta_{3}
\end{array}\right)
$$

Staucht man nun die fcc-Zelle um $\eta_{2}=-0,83$ und dehnt gleichzeitig die anderen beiden Achsen um $\eta_{1}=\eta_{3}=1,12$, erhält man eine kubisch 
raumzentrierte Zelle, deren Volumen nur um den Faktor $\eta_{1} \cdot \eta_{2} \cdot \eta_{3}=1,04$ von der ursprünglichen fcc-Zelle abweicht. Wegen der Forderung einer unter der Transformation invarianten Habitusebene müssen zusätzlich zu $B$ noch reine Drehungen zugelassen werden.

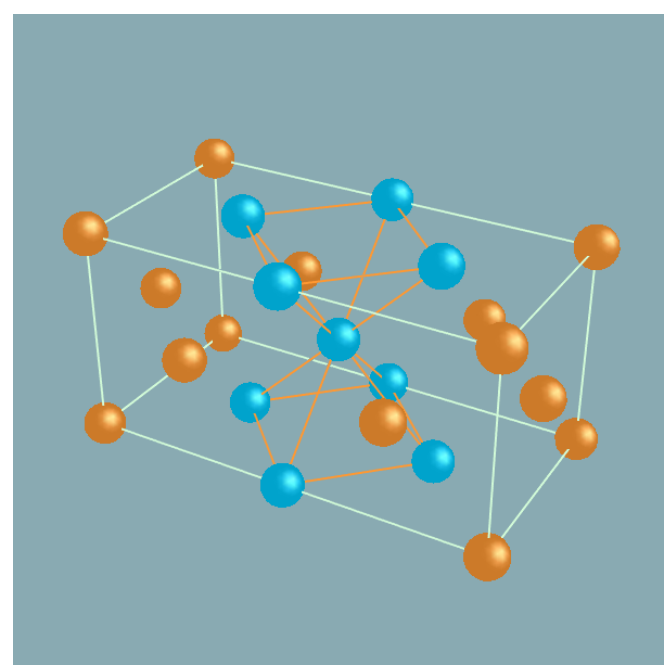

(a) fcc-Struktur

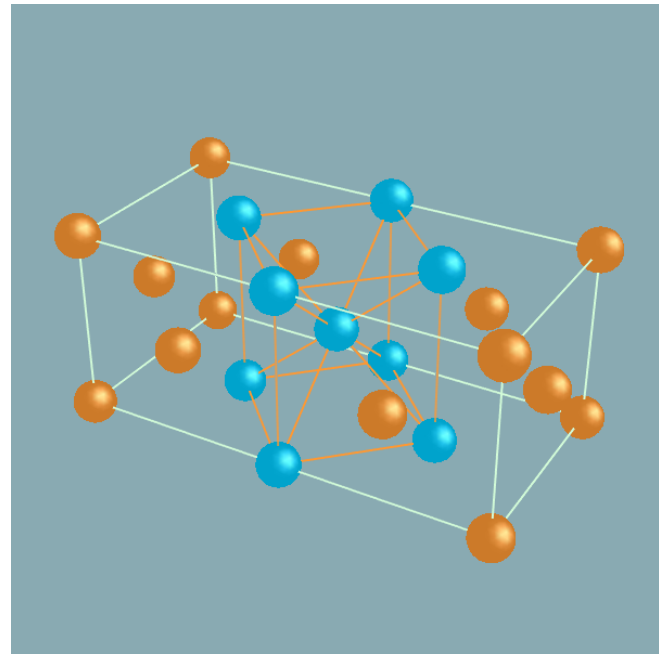

(c) bct-Struktur

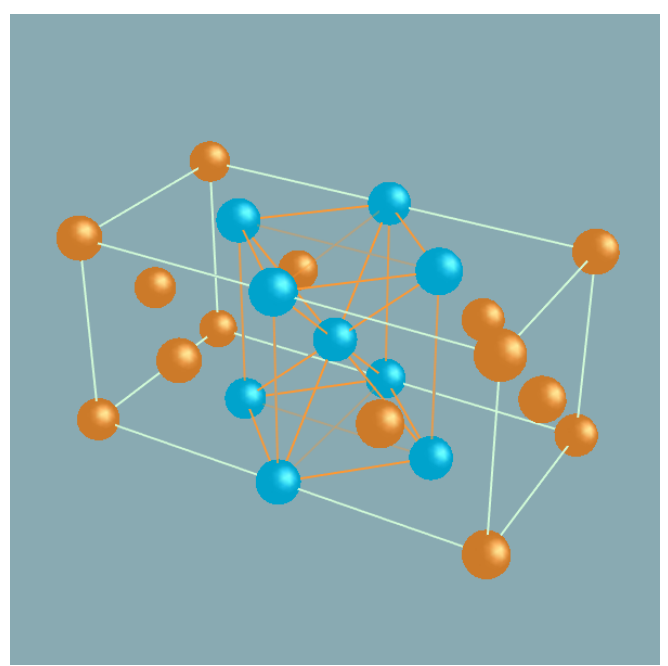

(b) fct-Struktur

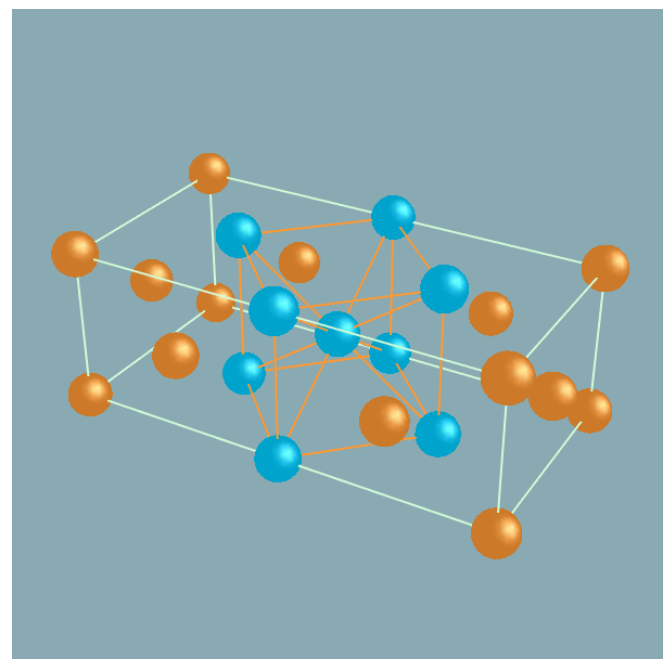

(d) bcc-Struktur

Abbildung 2.3: Bain Pfad [12]: Oben links ein unverzerrtes fcc-Gitter, bei dem die Verzerrung der blau dargestellten raumzentrierten Einheitszelle $c / a=$ 1,41 beträgt, oben rechts ein fct-Gitter mit $c / a=1.33$, unten links ein bctGitter mit $c / a=1.2$ unten rechts ein unverzerrtes bcc-Gitter mit $c / a=1.0$. 

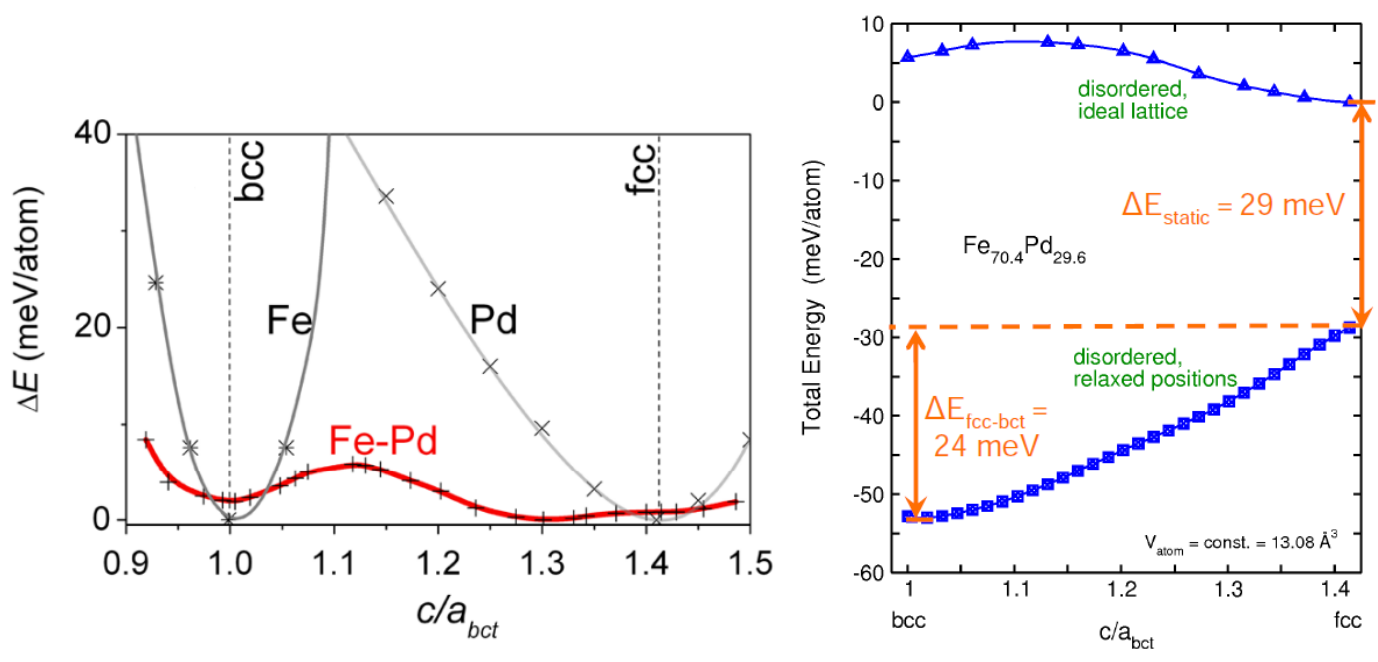

Abbildung 2.4: Energieunterschiede pro Atom beim Dehnen eines ungeordneten $\mathrm{Fe}_{70} \mathrm{Pd}_{30}$ Kristalls. Links: die Atome sind auf ihren idealen Positionen fixiert, aus [13]. Rechts: Die Atome können zusätzlich ihre Anordnung optimieren, aus [15].

In Volumenproben beobachtet man oft nur Übergänge, die nicht den gesamten Bain-Pfad abbilden, sondern eine kleinere Verzerrung aufweisen. Je nach dem, ob die Struktur eher als eine leicht verzerrte bcc-Struktur oder eine leicht verzerrte fcc-Struktur beschrieben werden kann, werden die beiden Begriffe fct und bct verwendet. Dabei gibt es aus kristallographischer Sicht keinen Unterschied zwischen fct und bct, da beide Gitter die selbe Symmetrie aufweisen. Abbildung 2.3 zeigt die in der Literatur verwendeten Bezeichnungen: zunächst die fcc-Struktur mit $c / a=1,41$, die fct-Struktur mit $c / a=1,33$ [36, 37], die bct-Struktur mit $c / a<1,3$ [21] und schließlich die bcc-Struktur mit $c / a=1$.

Für das System $\mathrm{Fe}_{70} \mathrm{Pd}_{30}$ zeigen quantenmechanische Rechnungen, dass die Energieunterschiede zwischen der fcc- und der bcc-Struktur entlang des Bain-Pfades sehr gering sind [13]. Hierfür wurden alle Atome auf ihren idealen Positionen fixiert, und für jede Konfiguration die Gesamtenergie bestimmt. Man erkennt im linken Teil von Abbildung 2.4 einen sehr starken Anstieg der Energie pro Atom sowohl für elementares Eisen als auch für elementares Palladium und einen sehr flachen Verlauf für die $\mathrm{Fe}_{70} \mathrm{Pd}_{30}$-Legierung, die ein Minimum im fct-Bereich und ein weiteres Minimum bei der bcc-Struktur aufweist.

Neuere Rechnungen, deren Ergebnisse im rechten Teil von Abbildung 2.4 dargestellt sind, zeigen darüber hinaus einen großen Einfluss der Atomposi- 
tionen [15]. Hierfür wurde für jede betrachtete Verzerrung des Gitters eine Superzelle mit 108 Atomen zusätzlich mit einem Conjugate-GradientAlgorithmus [38] relaxiert. Hierbei wird den Atomen erlaubt, sich in die energetisch günstigsten Positionen zu bewegen, so dass die Energie pro Atom insgesamt zwischen $29 \mathrm{meV}$ und $53 \mathrm{meV}$ tiefer liegt. Im Gegensatz zu den unrelaxierten Atompositionen, die einem ähnlichen Verlauf wie den Ergebnissen aus [13] folgen, erhält man nun kein Energieminimum mehr für die fct-Struktur, sondern nur ein einziges Minimum bei einer schwach verzerrten bct-Struktur. Allen dieser quantenmechanischen Rechnungen ist es gemein, dass sie bei $T=0$ durchgeführt werden, und daher weder thermische Fluktuationen noch den Einfluss der Entropie bei endlichen Temperaturen wiedergeben können.
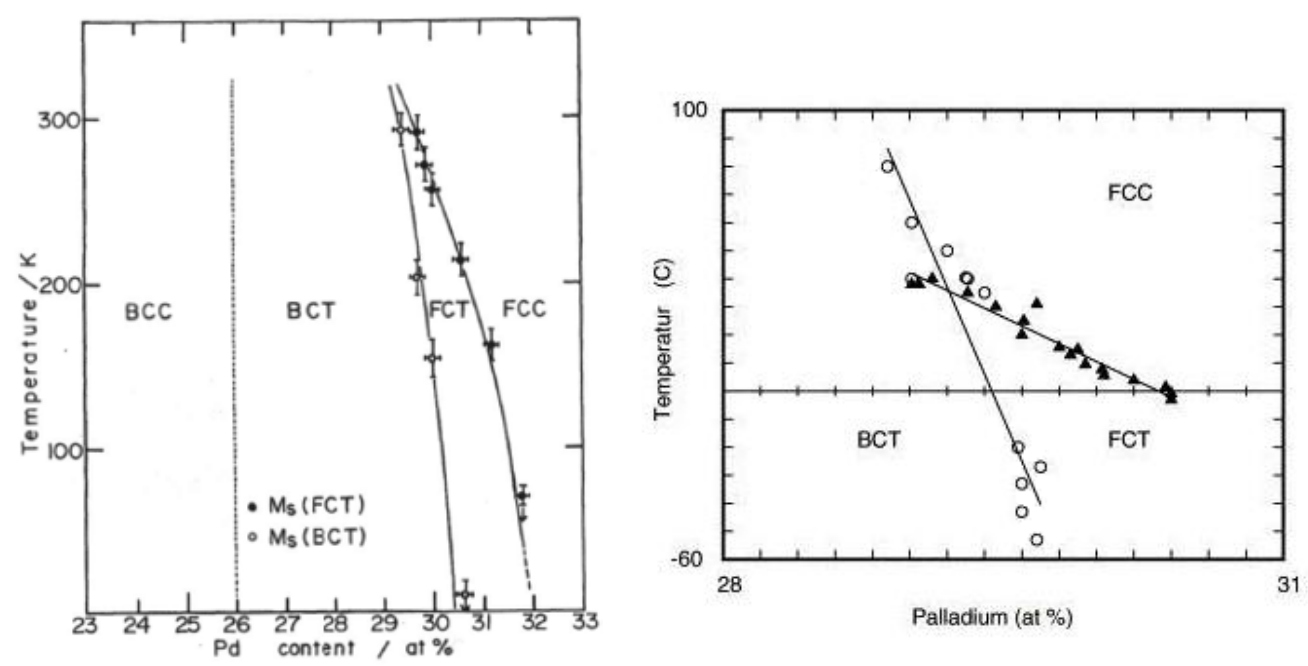

Abbildung 2.5: Metastabile Phasendiagramme für Bulk FePd-Einkristalle: links aus [21] und rechts aus [39].

Experimentelle Befunde in Abbildung 2.5 zur Gitterstruktur zeigen ein etwas anderes Bild: Hier ist es nicht möglich, die Energie verschiedener Strukturen direkt miteinander zu vergleichen, stattdessen wird beobachtet, welche Struktur sich bei welcher Zusammensetzung und Temperatur einstellt. Dies muss nicht notwendigerweise die energetisch günstigste aller Strukturen sein, sondern stellt nur die günstigste kinetisch zugängliche Struktur dar. Insbesondere der von Cui beobachtete fcc-bct Übergang für Palladiumkonzentrationen unter 29,7\% und Temperaturen unter $30^{\circ} \mathrm{C}$ wird in der Literatur als nicht thermoelastisch und irreversibel bezeichnet [21,39]. Dies wird dadurch erklärt, dass der Übergang mit einer thermischen Hysterese von einigen hundert Grad einhergeht, und die Rücktransformation erst bei Temperaturen 
auftreten würde, bei denen die Probe schon entmischt, so dass eine Rücktransformation bisher nicht beobachtet werden konnte.

Eine solche Hysterese ist bei Martensitumwandlungen immer zu erwarten, ein typischer Verlauf ist in Abbildung 2.6 dargestellt. Eine martensitische Umwandlung kann thermodynamisch als Phasenübergang erster Ordnung beschrieben werden [40]. Tatsächlich stattfinden kann dieser Übergang vom Austenit zum Martensit allerdings erst dann, wenn die Differenz der Gibbschen freien Enthalpien $G(T, p, n)$ der beiden Phasen (die in diesem Zusammenhang auch als die chemische Energie $G_{c}$ bezeichnet wird) die aufzuwendende nicht-chemische Energie $G_{n c}$ überschreitet, in der die Beiträge der inneren Reibung, der neu zu bildenden Grenzflächen, und der aufzuwendenden elastischen Verzerrung der Matrix zusammengefasst sind:

$$
\begin{aligned}
G_{c} & =G_{\text {Austenit }}-G_{\text {Martensit }} \\
G_{n c} & =\Delta G_{\text {elastisch }}+\Delta G_{\text {Grenzflächen }}+\Delta G_{\text {Reibung }}
\end{aligned}
$$

Dies führt dazu, dass die Umwandlung nicht bei der Temperatur stattfindet, bei der die beiden Gibbschen freien Enthalpien gleich sind, sondern eine gewisse Unterkühlung bzw. Überhitzung notwendig ist. Der Beitrag der elastischen Verzerrung ist allerdings während der Umwandlung nicht konstant, sondern nimmt mit zunehmendem Martensitanteil zu. Dies führt dazu, dass die Martensitbildung einsetzt sobald die Unterkühlung ausreicht, um die anfängliche nicht-chemische Energie zu kompensieren, aber eine stärkere Unterkühlung notwendig ist, um die Transformation vollständig abzuschließen. Bei der Umwandlung vom Martensit zum Austenit tritt dieser Effekt ebenfalls auf, da hier die elastische Energie wieder frei wird. Die Umwandlung beginnt also bei geringeren Überhitzungen, da hier viel elastische Energie frei wird; um sie zu vervollständigen ist allerdings eine höhere Überhitzung notwendig.

Die martensitische Umwandlung wird also durch vier Temperaturen charakterisiert: die Temperatur bei der sich der Martensit zu bilden beginnt $\left(M_{s}\right)$, die Temperatur bei der die Bildung des Martensits vollständig abgeschlossen ist $\left(M_{f}\right)$, sowie die Start- und Endtemperatur der Austenitbildung $A_{s}$ und $A_{f}$. Hierbei wird die Breite der Hysterese, also die Differenz zwischen $M_{f}$ und $A_{s}$ bzw. $A_{f}$ und $M_{s}$ durch die innere Reibung, die bei der Bewegung einer Phasengrenzfläche durch das Gitter entsteht und durch die Entstehung von Gitterdefekten bei der Umwandlung verursacht. Die Differenz zwischen Start- und Endtemperatur einer Umwandlung wird von der elastischen Energie der sich bei der Umwandlung bildenden Verzerrungsfelder verursacht $[40,41]$.

Die Temperatur, bei der die Umwandlung stattfindet, lässt sich durch einen angelegten Druck bzw. eine mechanische Spannung verschieben, da 


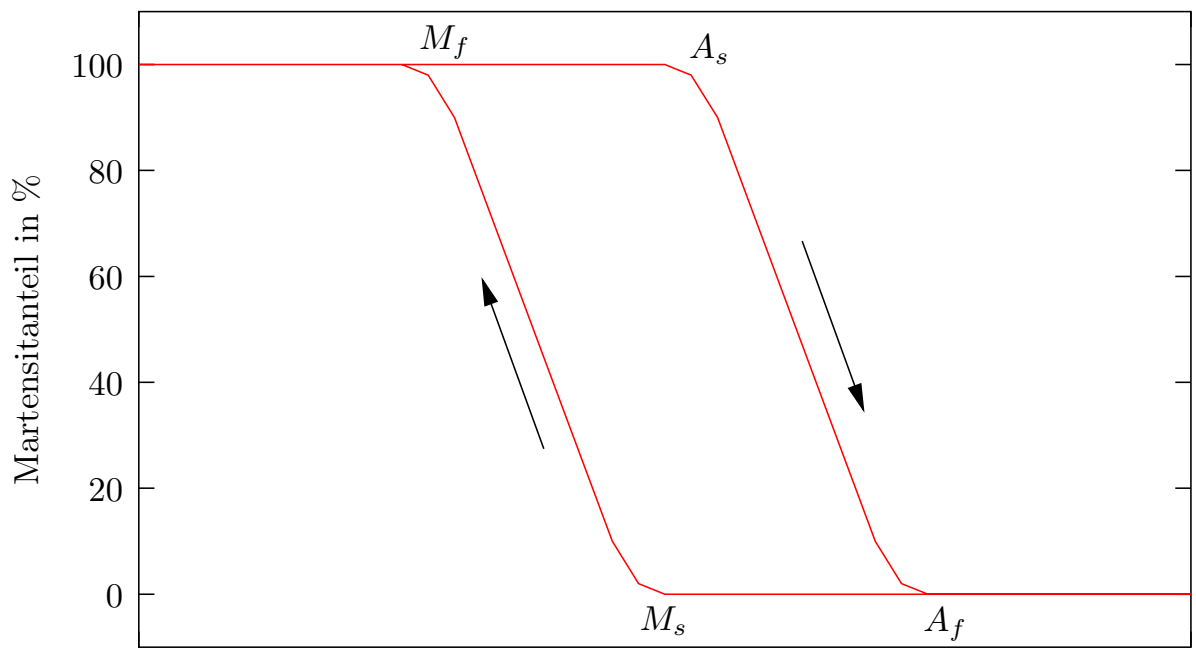

Temperatur

Abbildung 2.6: Typischer Verlauf des Anteils der Martensitphase in Abhängigkeit der Temperatur für eine Formgedächtnislegierung.

diese die freiwerdende bzw. aufzuwendende elastische Energie verändert. Dieser Zusammenhang lässt sich mit einer modifizierten Clausius-ClapeyronGleichung durch die Enthalpie- und Volumenänderung beim Übergang beschreiben [40]:

$$
\frac{\mathrm{d} p}{\mathrm{~d} T}=\frac{\Delta S}{\Delta T}=\frac{\Delta H}{T \Delta V}
$$

\subsubsection{Der Invar-Effekt}

Schon lange ist bekannt, dass Eisen-Nickel-Legierungen ein anomales Verhalten ihrer thermischen Ausdehnung und vieler anderer Größen wie den elastischen Moduli oder der spezifischen Wärme zeigen [42, 43]. Diese Effekte sind nicht auf das System Eisen-Nickel beschränkt, sondern treten auch in vielen anderen ungeordneten fcc-Legierungen auf. Der Name Invar, der nicht für den Effekt sondern auch für die Legierung $\mathrm{Fe}_{65} \mathrm{Ni}_{35}$ geprägt wurde, stammt daher, dass in einem gewissen Temperaturbereich die thermische Ausdehnung fast vollständig verschwindet.

Vor diesem Hintergrund verwundert es nicht, dass Eisen-Palladium auch einen ausgeprägten Invar-Effekt zeigt [3, 20, 23], zumal Palladium im Periodensystem genau unter Nickel steht und ihm deshalb in vielen elektronischen Eigenschaften stark ähnelt. Es ist schon lange klar, dass der Ma- 
gnetismus eine entscheidende Rolle beim Verständnis dieses Effekts spielen muss $[44,45,46]$. Hierbei wurde unter anderem ein Modell vorgeschlagen, dass einen Übergang zwischen verschiedenen magnetischen Zuständen und eine damit verbundene Volumenänderung beinhaltet, die dann der thermischen Ausdehnung entgegenwirkt [44]. Neuere Erklärungen gehen von einer Änderung der magnetischen Struktur aus, bei der der Spin der Atome nicht mehr nur parallel oder antiparallel ausgerichtet wird, sondern teilweise auch ungeordnet vorliegen kann [47].

\subsubsection{Instabilität des Kristallgitters}

Alle diese Erklärungen setzen allerdings voraus, dass die magnetischen Kräfte in der selben Größenordnung liegen wie die Ursache der thermischen Ausdehnung - die Asymmetrie des interatomaren Potentials - um diese kompensieren zu können. Bei den meisten Materialien ist dies nicht der Fall. Das interatomare Potential wird im Wesentlichen von elektrostatischen Kräften bestimmt, die in den meisten Fällen sehr viel stärker als die magnetischen Wechselwirkungen sind. Bei den Invar-Legierungen kommt nun ein weiterer Effekt zum tragen: Das Phononenspektrum weist so genannte soft modes auf, also Schwingungsmoden, bei denen mit geringer Energie große Amplituden erreicht werden. Das Gitter wird also weich [48], vorallem in der $\mathrm{T}_{2}$-Mode [19]. Dieses Erweichen der Struktur wird als Vorstufe für den Martensitübergang gesehen [43].

In den elastischen Konstanten von $\mathrm{Fe}_{70} \mathrm{Pd}_{30}$ zeigt sich, dass vor allem $C^{\prime}$ in der Nähe des martensitischen Übergang stark abnimmt [19, 49]. Diese Konstante beschreibt die Steifigkeit eines Gitters gegen eine Scherung der $\{110\}$-Ebenen in $\langle 1 \overline{1} 0\rangle$-Richtung. Die Umwandlung der fcc-Struktur in die fct-Struktur kann so durch zwei solcher gekoppelter Scherprozesse beschrieben werden [50].

Als mikroskopische Ursache für diese strukturelle Instabilität wird ein Band-Jahn-Teller-Effekt angenommen [51], der auf eine erhöhte Zustandsdichte an der Fermikante zurückgeführt wird, die durch die martensitische Umwandlung reduziert wird [52].

\subsubsection{Magnetostriktion}

Eisen-Palladium zeigt wie viele andere Metalle und Legierungen auch eine Magnetostrikion [19]. Dies setzt voraus, dass nicht alle bindenden Orbitale eines Atoms äquivalent sind, sondern diese Entartung zumindest teilweise (zum Beispiel durch die Kristallfeldaufspaltung) aufgehoben ist und die Atome dadurch leicht deformiert sind. In Systemen mit einer starken Spin-Bahn- 


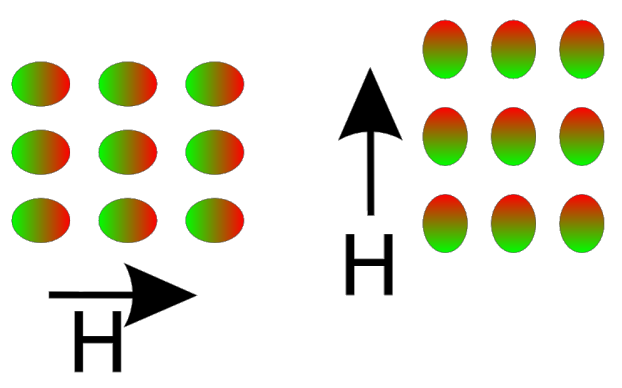

\begin{abstract}
Abbildung 2.7: Skizze zur Magnetostriktion: Durch die Spin-BahnWechselwirkung werden die Deformationen der Atome in einem Magnetfeld ausgerichtet, was je nach Richtung des Magnetfelds zu einer Änderung der Probenabmessungen führen kann.
\end{abstract}

Wechselwirkung ist nun diese Deformation der Atome an die Richtung der Magnetisierung gekoppelt, und im entmagnetisierten Zustand regellos verteilt. Wird das Material nun magnetisiert, richten sich diese Deformationen der Orbitale ebenfalls alle aus. Eine Änderung der Magnetisierung ändert so die atomaren Abstände und damit in letzter Konsequenz auch die äußeren Abmessungen einer Probe, wie in Abbildung 2.7 dargestellt. Typische relative Längenänderungen liegen hierbei je nach Materialsystem zwischen $10^{-8}$ und $10^{-4}$ [53]. Bei der Magnetostriktion werden nur die magnetischen Domänen verändert, eventuell in dem Material vorhandene Zwillingsgrenzen bleiben dagagen unverändert.

\title{
2.1.5 Thermischer und magnetischer Formgedächtnis- effekt
}

Die schon beim Invar-Effekt diskutierte Eigenschaft des Atomgitters, dass gewisse Schwingungsmoden sehr leicht zu großen Amplituden angeregt werden können und dadurch das Gitter sehr weich sein kann, beeinflusst nicht nur die mikroskopischen Eigenschaften sonder führt auch dazu, dass sich FePdProben durch verschiedene Effekte makroskopisch leicht verformen lassen.

Sowohl beim thermischen wie auch magnetischen Formgedächtniseffekt wird im Gegensatz zur Magnetostriktion die Ausrichtung von Zwillingsvarianten verändert, so dass hier potentiell sehr viel größere Längenänderungen möglich sind. Für den thermischen Formgedächtniseffekt nutzt man die Änderung der Symmetrie des Gitters beim Martensitübergang aus. Wie in Abbildung 2.8 dargestellt, behält ein Kristall seine makroskopische Form bei 


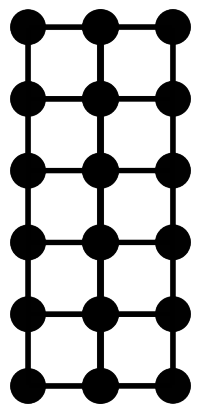

(a)

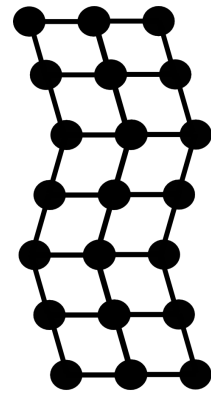

(b)

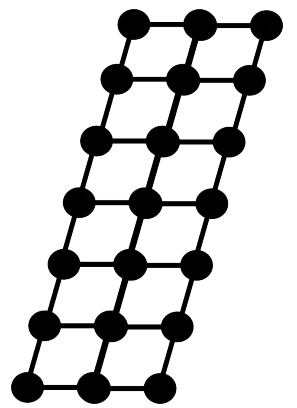

(c)

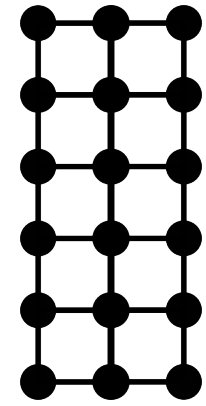

(d)

Abbildung 2.8: Thermischer Formgedächtniseffekt: Die Struktur geht zunächst durch Abkühlen vom kubischen Austenit in den verzwillingten Martensit über. Dieser kann verformt werden, in dem die Zwillingsgrenzen verschoben werden. Beim Erwärmen stellt sich wieder der ursprüngliche Austenit ein.

und kompensiert die Änderung der Gitterstruktur durch die Bildung von Zwillingen, wenn er vom Austenit zum Martensit abgekühlt wird. Wird der Martensit nun deformiert, bewegen sich diese Zwillinge durch den Kristall, ohne dass weitere Defekte gebildet werden müssen. Wird das Material nun wieder in den austenitischen Zustand erwärmt, existiert der Freiheitsgrad der Zwillinge nicht mehr, so das sich das Material wieder in seine ursprüngliche Form zurück verformt. Außer diesem Formgedächtniseffekt lässt sich der Martensitübergang auch für weitere interessante mechanische Eigenschaften nutzen: Zum einen kann das Material im martensitischen Zustand über einen weiten Bereich plastisch verformt werden, in dem die Zwillingsgrenzen bewegt werden (man spricht hierbei von Superplastizität), zum anderen kann die Martensitumwandlungstemperatur durch mechanische Spannungen beeinflusst werden (siehe Gleichung 2.4). Wird das Material knapp oberhalb der Umwandlungstemperatur stark deformiert, wandelt es sich der Austenit in Martensit um, und lässt sich dadurch relativ leicht verformen. Entlastet man das Material wieder, erhält man wieder den Austenit und die ursprüngliche Form, daher spricht man hier von Superelastizität.

Der magnetische Formgedächtniseffekt läuft dagegen vollständig in der martensitischen Phase ab. Hierbei wird die große magnetische Anisotropie des Martensits ausgenutzt. Legt man an einen fct-Fe ${ }_{70} \mathrm{Pd}_{30}$-Kristall ein Magnetfeld an, dessen Richtung nicht mit einer leichten Achse der Magnetisierung (den beiden langen $a$-Achsen [39]) zusammenfällt, werden dadurch die Zwillingsgrenzen bewegt, so dass die Gitterzelle in eine andere Zwillingsvariante umklappt bei der die leichte Richtung der Magnetisierung mit der Richtung 


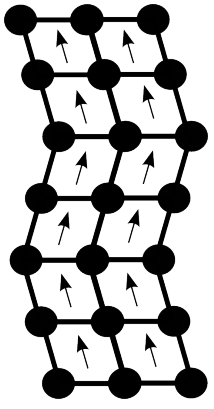

(a)

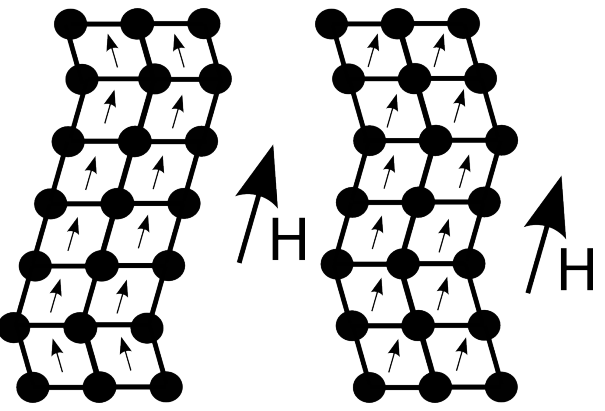

(b)

(c)

\begin{abstract}
Abbildung 2.9: Magnetischer Formgedächtniseffekt: Ohne äußeres Magnetfeld sind alle Spins der Gitterzellen entlang der leichten Achse (hier die lange Gitterachse) ausgerichtet (linkes Bild). Durch ein externes Magnetfeld können die Zwillingsvarianten umorientiert werden (mittleres Bild). Nur wenn die Beweglichkeit der Zwillingsgrenzen nicht groß genug ist, wird die Magnetisierung aus der leichten Richtung herausgedreht (rechtes Bild).
\end{abstract}

des äußeren Felds übereinstimmt (siehe Abbildung 2.9). Für diesen Effekt werden in der Literatur außer dem Begriff MSM (Magnetic Shape Memory) teilweise auch die Begriffe MIR (Magnetic Induced Reorientation) und MFIS (Magnetic Field Induced Strain) verwendet. Bei der Reorientierung der Zwillingsvarianten wird ein Teil der Anisotropieenergie für die mit diesem Umklappen verbundene effektive Reibung aufwendet. Wird das äußere Magnetfeld nun entfernt, verbleibt der Kristall in dieser neuen Konfiguration und kann entweder durch mechanische Spannungen oder durch ein geeignetes Magnetfeld mit anderer Ausrichtung wieder zurück verformt werden. Dies setzt voraus, dass die Zwillingsgrenzen in dem Kristall leicht beweglich sind, die magnetische Anisotropieenergie also größer als die zu überwindende Reibung ist. Sind die einzelnen Martensitvarianten zu stark gepinnt, wird stattdessen die Magnetisierung aus der leichten Achse heraus gedreht (ebenfalls in Abbildung 2.9 dargestellt).

\title{
2.2 Wachstum dünner Filme
}

Betrachtet man das extrem frühe Stadium der Deposition eines Films auf einem Substrat, so haften zunächst nur einzelne Atome auf dem (im Idealfall) atomar glatten Substrat. Diese einzelnen Adatome haben eine sehr geringe Zahl an Nachbarn und sind dementsprechend schwach gebunden. Sie können sich daher durch Oberflächendiffusion leicht bewegen, und werden 
sich bevorzugt aneinander anlagern, da dadurch die Zahl der Nachbarn und damit die Zahl der Bindungen erhöht werden kann. Für ein in Abbildung 2.10

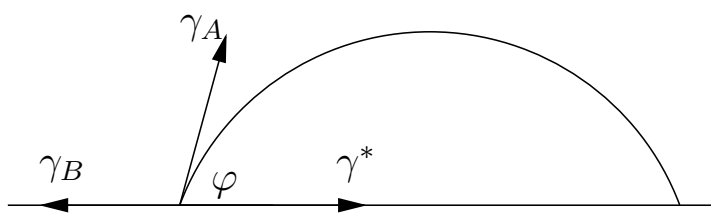

Abbildung 2.10: Kräftegleichgewicht beim Benetzen einer Oberfläche.

dargestelltes tropfenförmiges Aggregat auf einer glatten Substratoberfläche gilt folgendes Gleichgewicht zwischen der freien Energie der Substratoberfläche $\left(\gamma_{B}\right)$, der Freien Energie der Oberfläche des Schichtmaterials $\left(\gamma_{A}\right)$, der Grenzflächenenergie $\gamma^{*}$ und dem Kontaktwinkel $\varphi$ :

$$
\gamma_{B}=\gamma^{*}+\gamma_{A} \cos \varphi
$$

Somit erhält man nur für bestimmte Kombinationen der Grenzflächenenergien einen stabilen Kontaktwinkel, andernfalls stellt sich Lagenwachstum ein, das als Grenzfall $\varphi=0$ verstanden werden kann.

Nach der Art des Wachstums werden drei Wachstumsmoden dünner Filme unterschieden, die in Abbildung 2.11 dargestellt sind [54]:

- Beim Frank-van-der-Merve-Wachstum wächst der Film in einzelnen Atomlagen. Zunächst verteilen sich alle Adatome so, dass sie das Substrat mit einer Monolage bedecken, auf dieser wieder atomar glatten Schicht wächst danach ebenso die nächste Lage auf. Diese Fall tritt auf bei $\varphi=0$ und damit bei $\gamma_{B} \geq \gamma^{*}+\gamma_{A}$

- Beim Volmer-Weber-Wachstum lagern sich die einzelnen Adatome zu dreidimensionalen Inseln zusammen. Weitere Adatome diffundieren über das Substrat, bis sie entweder eine neue Insel bilden oder sich an eine bestehende Insel anlagern. Das Substrat wird zunächst nicht vollständig bedeckt, erst bei hohen Schichtdicken koaleszieren die einzelnen Inseln zu einer geschlossenen Schicht. Dieser Fall tritt auf bei $\varphi>0$ und damit bei $\gamma_{B}<\gamma^{*}+\gamma_{A}$

- Das Stranski-Krastanov-Wachstum stellt eine Mischform zwischen Lagenund Inselwachstum dar. Hier bildet sich zunächst eine oder mehrere geschlossene Monolagen, auf der dann dreidimensionale Inseln aufwachsen. 


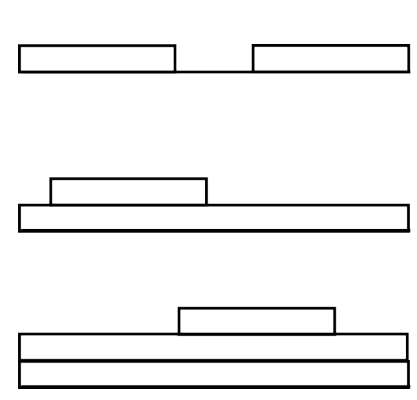

(a)

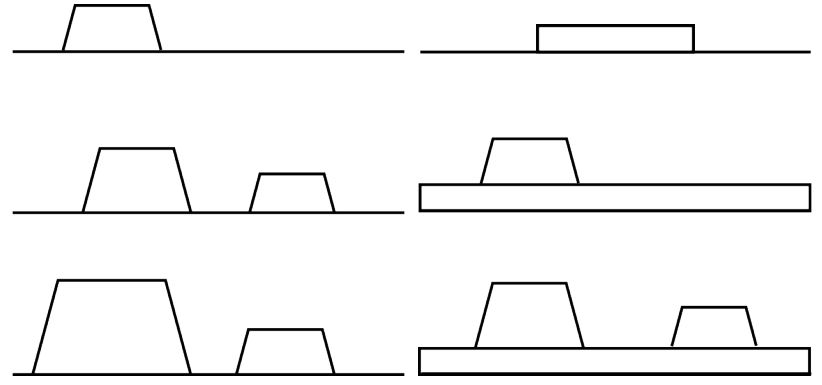

(b) (c)

Abbildung 2.11: Die drei Wachstumsmoden: Lagenwachstum (a), Inselwachstum (b) und Stranski-Krastanov-Wachstum (c)

Auch die Übersättigung der Dampfphase beeinflusst die Wachstumsmode, so dass bei hohen Übersättigungen eher Lagenwachstum vorherrscht, da oberhalb einer kritischen Übersättigung die freie Enthalpie zur Bildung zweidimensionaler Keime geringer ist als zur Bildung dreidimensionaler Keime [28].

Ein Stranski-Krastanov-Wachstum kann sich unter anderem dann ergeben, wenn zwar die Grenzflächenenergie ein Lagenwachstum erzeugen würde, die Gitterkonstanten von Substrat und Film jedoch stark unterschiedlich sind. Die erste Atomlage passt sich in ihrer Gitterkonstante an das Substrat an, aber für jede weitere Lage muss eine elastische Energie für die Gitterverzerrung aufgewendet werden. Wenn die Adhäsionskräfte nun eine geringere Reichweite als die elastischen Kräfte aufweisen, wird ein Übergang vom zweidimensionalen zum dreidimensionalen Wachstum auftreten.

Auch die Substrattemperatur hat einen großen Einfluss auf die Morphologie des wachsenden Films: Bei geringen Substrattemperaturen ist auch die Diffusionslänge der Adatome auf der Oberfläche gering, so dass sich viele kleine Keime bilden, während bei einer hohen Substrattemperatur weniger und dafür größere Keime auf dem Substrat zu erwarten sind. Nach dem Zonenmodell von Thornton [55, 56], das für polykristalline Filme im Grenzfall hoher Depositionsraten entwickelt wurde, ist für Substrattemperaturen $\left(T_{s}\right)$ unter einem Drittel der Schmelztemperatur des deponierten Materials $\left(T_{m}\right)$ eine sehr defektreiche Struktur zu erwarten. In diesem unteren Temperaturbereich ist Abschattung der dominierende Effekt, so dass die Struktur des Films von sehr kleinen Körnern, die schon bestehende Körner überwachsen, sowie vielen Korngrenzen und Leerstellen geprägt ist. Für Substrattemperaturen im Bereich 0,3< $T_{s} / T_{m}<0,5$ ist nach diesem Modell Oberflächendiffusion der dominierende Prozess. Daher erwartet man hier ein säulenartiges 
Wachstum, bei dem im Wesentlichen die in der Anfangsphase des Wachstums gebildeten Körner in ihrer Höhe weiter wachsen. Für noch höhere Temperaturen ist die Struktur von starker Rekristallisation geprägt und weist große Kristallite auf.

Im Allgemeinen wachsen dünne Schichten als Polykristalle auf, da sich auf dem Substrat verschiedene Keime bilden, die dann zu einer geschlossenen Schicht zusammenwachsen. Diese Keime sind oft nicht völlig regellos orientiert, so dass der Film eine Textur aufweist. Bei vielen fcc-Metallen beobachtet man eine (111)-Drahttextur, bei der die (111)-Ebenen bevorzugt parallel zum Substrat ausgerichtet sind, die einzelnen Kristallite aber in der Substratebene regellos gegeneinander gedreht sind. Diese Textur kann durch die Struktur der Keime in der frühesten Phase des Wachstums erklärt werden [57]: Es ist energetisch günstiger, dass sich die ersten Atome eines Keims möglichst nahe beieinander positionieren. Wächst dieser Keim nun weiter, erhält man auf dem Substrat eine dicht gepackte hexagonale Struktur, die bei einem fcc-Kristall gerade die (111)-Ebene bildet. Um im Gegensatz dazu zum Beispiel eine (100)-Textur zu erhalten, müsste die unterste Atomlage ein quadratisches Gitter bilden, das sehr viel weniger dicht gepackt wäre.

Nicht nur die Wechselwirkung der deponierten Atome untereinander, sondern auch die Gitterstruktur des Substrats kann die Struktur des wachsenden Films massiv beeinflussen. So ist es für eine Monolage eines Films auf einem einkristallinen Substrat zu erwarten, dass die Adatome sich so anordnen, dass sie ihre Energie in dem an der Oberfläche herrschenden Potential, das die Periodizität der Substratatome wiedergibt, minimieren. Wächst die Schicht nun mit exakt der Gitterkonstante des Substrats auf, spricht man von pseudomorphem Wachstum. Je nach Verhältnis des Substrat-Gitterparameters zum Gitterparameter der Schicht wird hierbei die Schicht jedoch elastisch verzerrt, so dass in späteren Atomlagen diese Verzerrung durch den Einbau von Versetzungen (so genannte misfit dislocations) abgebaut wird. Ist der Unterschied der Gitterkonstanten zu groß, ist statt eines pseudomorphen Wachstums der untersten Atomlagen eher die Bildung von Versetzungen direkt an der Substrat-Film-Grenzfläche zu erwarten.

Von epitaktischem Wachstum spricht man, wenn eine feste Orientierungsbeziehung zwischen dem Substrat und dem Film existiert. Diese Beziehung wird durch Angabe der in Schicht und Film parallel liegenden Ebenen und der in diesen Ebenen parallel zueinander liegenden Richtungen charakterisiert:

$$
(H K L)_{\text {Substrat }}\left\|(h k l)_{\text {Film }} ;[U V W]_{\text {Substrat }}\right\|[u v w]_{\text {Film }}
$$

Ein solches epitaktisches Wachstum wird für viele Materialien nur oberhalb einer kritischen Substrattemperatur und unterhalb einer kritischen Rate 


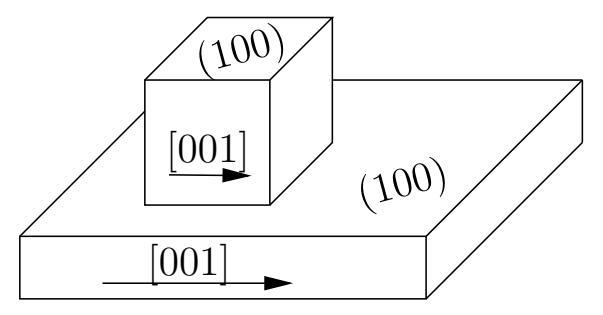

Abbildung 2.12: Beispiel für eine epitaktische (100)\|(100); [001]\|[001] Beziehung, die auch als cube-on-cube-Struktur bezeichnet wird .

beobachtet. Dies lässt sich im einfachsten Fall so deuten, dass die Atome einerseits beweglich genug sein müssen, um die idealen Positionen zu erreichen, und dieser Prozess andererseits eine gewisse Zeit dauert, in der die Atome nicht überwachsen werden dürfen [58].

\subsection{Ionenbestrahlung von Festkörpern}

Für die Wechselwirkung zwischen einem schnellen Ion und einem Festkörper spielen zwei verschiedene Effekte eine entscheidende Rolle: Zum einen die Wechselwirkung des Ions mit den Elektronen des Festkörpers, und zum anderen die Wechselwirkung des Ions mit den im Festkörper enthaltenen Atomkernen, die sich im einfachsten Fall als Zweikörperproblem beschreiben lässt. Die Wechselwirkung mit den Elektronen wird üblicherweise als eine kontinuierliche Reibung modelliert, die der unmittelbaren Umgebung der Ionenspur Energie zu führt, die dann von den Elektronen zumindest teilweise an das Atomgitter weitergegeben wird und somit die Probe in der Umgebung der Ionenspur stark aufheizt.

Die Wechselwirkung des Ions mit den Target wird dabei üblicherweise als stopping power bezeichnet, und als Energieverlust pro Länge, die das Ion im Festkörper zurücklegt, beschrieben:

$$
S(E)=\frac{\mathrm{d} E}{\mathrm{~d} x}
$$

Für fast alle Kombinationen aus Projektil- und Targetmaterial stehen hierfür sowohl theoretische Vorhersagen als auch tabellierte, aus Experimenten gewonnene Daten zur Verfügung [60]. Das Gewicht dieser verschiedenen Beiträge hängt dabei stark von der Energie des Ions ab. Für $\mathrm{Fe}_{70} \mathrm{Pd}_{30}$ dominieren unterhalb von $100 \mathrm{keV}$ die Stöße mit den Atomkernen, um $1 \mathrm{MeV}$ liefern beide Prozesse einen ähnlichen Beitrag und oberhalb von $10 \mathrm{MeV}$ überwiegt 


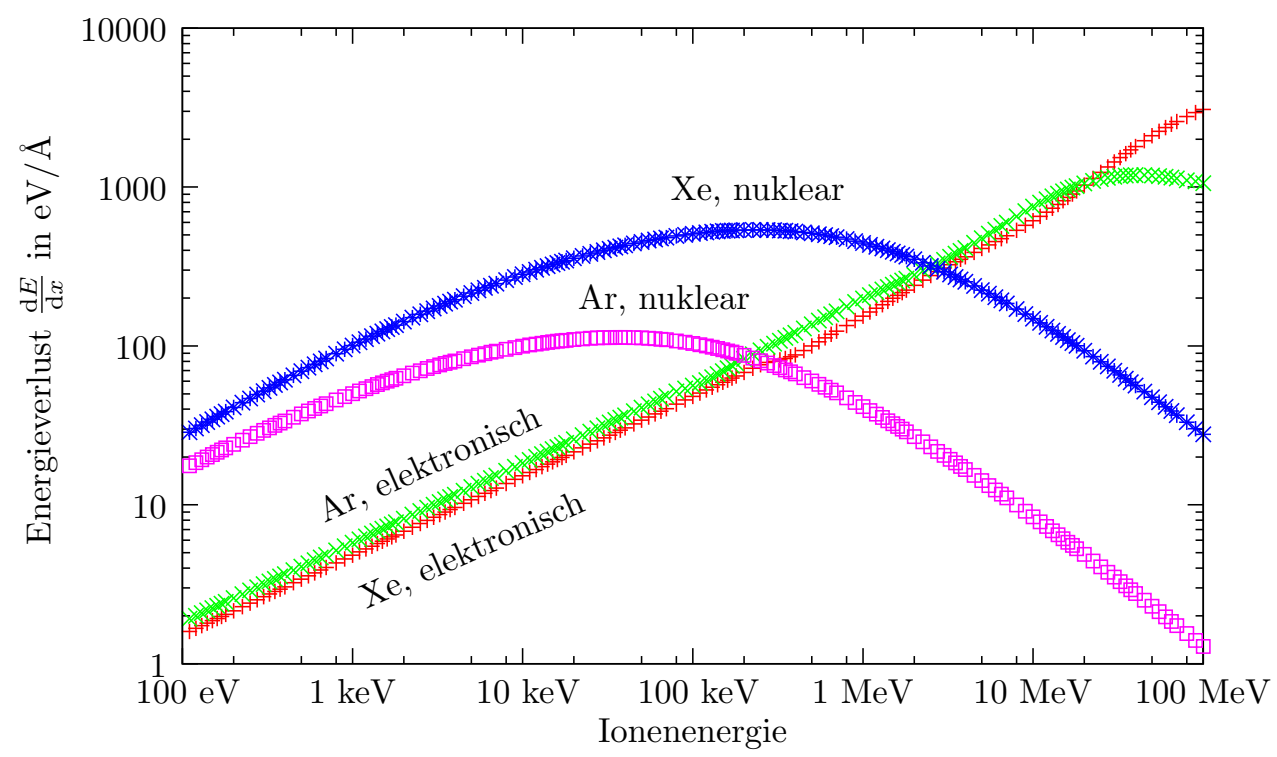

Abbildung 2.13: Simulationen mit SRIM [59]: Elektronischer und nuklearer Beitrag zur Energieverlust von Xenon- und Argonionen in $\mathrm{Fe}_{70} \mathrm{Pd}_{30}$

die Wechselwirkung mit dem Elektronensystem des Targets (siehe Abbildung 2.13).

Elastische Stöße zwischen einem Projektil (Masse $m_{p}$ ) und einem Targetatom (Masse $m_{t}$ ) lassen sich durch den kinematischen Faktor $k$ beschreiben, der die Energie des Projektils nach dem Stoß $\left(E_{1}\right)$ mit der Energie vor dem $\operatorname{Stoß}\left(E_{0}\right)$ verknüpft:

$$
E_{1}=k \cdot E_{0}
$$

Dieser Faktor lässt sich aus Impuls- und Energieerhaltungssatz bestimmen und beträgt [61]:

$$
k=\left(\frac{m_{p} \cos \Theta \pm \sqrt{m_{t}^{2}-m_{p}^{2}(\sin \Theta)^{2}}}{m_{p}+m_{t}}\right)^{2}
$$

Der Streuwinkel $\Theta$ wird hierbei im Laborsystem (und nicht im Schwerpunktsystem) gemessen. Ist die Masse des Targetatoms größer als die des Projektils, wird das positive Vorzeichen verwendet, anderenfalls das negative.

Bei jedem Stoß wird so ein Teil der Ionenenergie vom Projektil auf ein Targetatom übertragen. Ist dieser Energieübertrag nun kleiner als die Bindungsenergie des Atoms, wird es um seine Ruhelage schwingen und damit 
den Kristall lokal ebenfalls stark aufheizen. Überschreitet der Energieübertrag dagegen die Bindungsenergie, wird dieses Atom ebenfalls in Bewegung versetzt, hinterlässt an seinem ursprünglichen Ort eine Leerstelle und kann nun seinerseits wieder sowohl mit den Elektronen als auch mit den Atomkernen des Targets wechselwirken. Im Laufe der nun einsetzenden Kollisionskaskade werden so immer mehr mobile Atome erzeugt, die sich mit immer geringerer Energie durch das Target bewegen.

Alle diese Phänomene sind immer noch auf einen recht kleinen Bereich des Targets beschränkt und laufen auf sehr kurzen Zeitskalen ab, so dass insgesamt ein kleines Volumen die gesamte Energie des Projektils absorbiert hat und dabei sehr stark erwärmt wird. Eine Simulation mit SRIM [59] für $800 \mathrm{keV}$ Xe-Ionen, die auf ein $\mathrm{Fe}_{70} \mathrm{Pd}_{30}$-Target geschossen werden, zeigt in Abbildung 2.14, dass sich der Bereich, in dem es zu Kollisionen zwischen den Atomen kommt, bis in eine Tiefe von $200 \mathrm{~nm}$ erstreckt und einen Durchmesser von etwa $150 \mathrm{~nm}$ besitzt.

Das dadurch aufgeschmolzene Volumen gibt nun über Wärmeleitung seine Energie an das restliche Volumen des Targets ab, bis sich wieder ein thermisches Gleichgewicht eingestellt hat. Auch dieses Abkühlen läuft auf sehr kurzen Zeitskalen ab, so dass sich hier aus der Schmelze unter Umständen Nicht-Gleichgewichtsphasen entstehen können. So ist es möglich, Zwangslegierungen aus nicht mischbaren Metallen herzustellen, in dem elementare Multilagen bestrahlt werden (so genanntes Ionenmischen) oder auch kristalline Materialien (insbesondere Halbleiter) zu amorphisieren [62, 63, 64, 65].

In der Nähe der Oberfläche tritt ein weiterer Prozess auf - das Sputtern. Hierbei werden einzelne Atome aus dem Target gelöst, man spricht daher auch von Ionenstrahlzerstäuben. Die Sputterausbeute hängt stark von der Energie des Projektils ab: Für sehr geringe Energien wird an die Targetatome nicht genügend Energie übertragen, um sie aus dem Festkörper zu lösen; bei hohen Energien laufen die meisten Vorgänge tief unterhalb der Probenoberfläche ab, so dass hierbei ebenfalls die Sputterausbeute sehr niedrig ist. Wählt man die Ionenenergie niedrig genug, ist nicht nur die Sputterausbeute vernachlässigbar, sondern auch die Eindringtiefe der Ionen. Eine Simulation mit SRIM [59] für $0.1 \mathrm{keV}$ Ar-Ionen, die auf ein $\mathrm{Fe}_{70} \mathrm{Pd}_{30}$-Target geschossen werden zeigt in Abbildung 2.15, dass der von der Bestrahlung betroffene Bereich nur etwa einen Nanometer in das Target hineinreicht, und somit nur die obersten Atomlagen von der Ionenbestrahlung betroffen sind. Dies hat zur Folge, dass die Kinetik von Oberflächenprozessen wie Oberflächendiffusion oder viskoses Fließen an der Oberfläche durch die Bestrahlung stark verändert werde kann, die Kinetik von Volumenprozessen dagegen unverändert bleibt [66]. Diese Situation lässt sich durch eine effektive Oberfächentemperatur, die unter Umständen weit über der Temperatur des Volumens liegt, beschrei- 

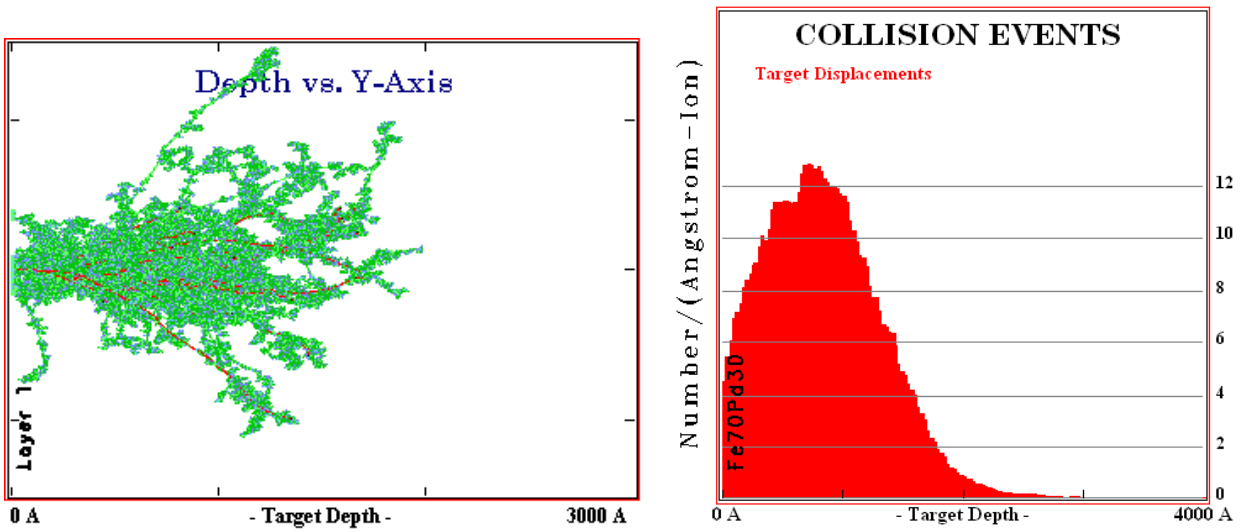

Abbildung 2.14: Simulationen mit SRIM [59]: Reichweite von $800 \mathrm{keV}$ XeAtomen in $\mathrm{Fe}_{70} \mathrm{Pd}_{30}$. Links sind jeweils in rot die Spuren der Projektile und in grün die Spuren der Targetatome dargestellt, rechts die Tiefenverteilung der Stoßereignisse.
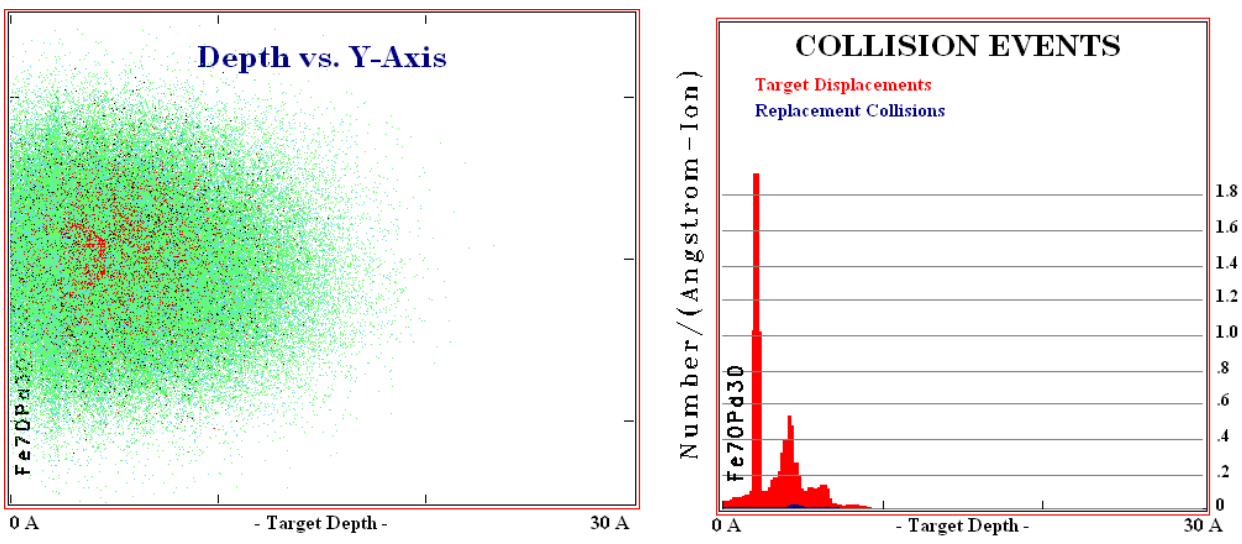

Abbildung 2.15: Simulationen mit SRIM [59]: Reichweite von $0.1 \mathrm{keV}$ ArAtomen in $\mathrm{Fe}_{70} \mathrm{Pd}_{30}$. Links sind jeweils in rot die Spuren der Projektiele und in grün die Spuren der Targetatome dargestellt, rechts die Tiefenverteilung der Stoßereignisse. 
ben. Dieser Effekt wird zum Beispiel bei der ionenstrahlunterstützten Deposition (IBAD) ausgenutzt. Neben einer erhöhten Adatombeweglichkeit [67] werden in der Literatur viele weitere Effekte diskutiert, wie die Erzeugung vom Punktdefekten an der Oberfläche [68], bevorzugtes Sputtern von Adatomen [69] oder das Entfernen von Atomen aus schon gebildeten Keimen [70]. Untersuchungen zu frühen Stadien der Deposition von Aluminium auf Isolatoren zeigen zum Beispiel eine durch IBAD vierfach erhöhte Zahl an Wachstumskeimen, während die Größe dieser Keime um den Faktor 5 bis 15 zurückgeht [69]. Platinfilme, die auf $\mathrm{Pt}(111)$-Einkristalle aufgedampft wurden zeigen für Substrattemperaturen von über $200 \mathrm{~K}$ mit IBAD ebenfalls mehr und kleinere Keime, während für Substrattemperaturen von $50 \mathrm{~K}$ statt amorpher Schichten epitaktisches Wachstum beobachtet wird [71]. 


\section{Kapitel 3}

\section{Experimentelle Methoden}

In diesem Kapitel sollen die verwendeten Methoden und Anlagen vorgestellt werden. Die Reihenfolge lehnt sich an den experimentellen Ablauf an, so werden zunächst die verschiedenen Methoden der Schichtherstellung und die Nachbehandlung der Schichten behandelt, und anschließend die Mess- und Charakterisierungsmethoden.

\subsection{Herstellung dünner Schichten}

Um das Wachstum dünner Eisen-Palladium-Filme umfassend zu untersuchen, wurden im Rahmen dieser Arbeit Proben mit drei verschiedenen Methoden hergestellt und charakterisiert. Der größte Teil der Proben wurde mittels Elektronenstrahlverdampfen hergestellt, wo hohe Temperaturen oder eine thermische Nachbehandlung notwendig sind, um die gewünschten Phasen zu erhalten. Im Gegensatz hierzu ist es mit der gepulsten Laserdeposition möglich, epitaktische Filme auch schon bei Raumtemperatur herzustellen. Beim ionenstrahlunterstützten Aufdampfen ist es möglich, den Einfluss von Oberflächenprozessen gegenüber im Volumen ablaufenden Prozessen zu variieren, um damit gezielt deren Einfluss zu untersuchen.

\subsubsection{Elektronenstrahlverdampfen}

Die zur Herstellung der meisten Proben verwendete Aufdampfanlage ist in Abbildung 3.1 schematisch dargestellt und wurde bereits in einigen anderen Arbeiten ausführlich beschrieben [72, 73], daher sollen hier nur die wichtigsten Charakteristika genannt werden. Über eine Schleusenkammer können Substrathalter in die eigentliche Aufdampfkammer transferiert werden, in der mittels Ionengetterpumpen ein typischer Druck von $3 \cdot 10^{-10}$ mbar erreicht 
wird. Die Aufdampfkammer ermöglicht somit die Präparation von Filmen hoher Reinheit, und enthält neben den Elektronenstrahlverdampfern unter anderem ein Massenspektrometer zur Restgasanalyse und einen Cryoschild, der einen großen Teil der Kammerwand umgibt. Durch das Befüllen dieses Cryoschilds mit flüssigem Stickstoff kann der Kammerdruck zusätzlich für einige Stunden um etwa eine Zehnerpotenz gesenkt werden.

Jeder der Verdampfer besitzt ein Filament, das durch einen Heizstrom Elektronen emittiert und das auf einem stark negativen Potential liegt. Der so erzeugte Elektronenstrahl wird nun durch ein Magnetfeld auf einen Kreisbogen gezwungen, in dessen Endpunkt der Tiegel mit dem zu verdampfenden Material $^{1}$ liegt. Zum Aufdampfen wird zunächst der Heizstrom langsam erhöht, um eine gleichmäßige Erhitzung des Materials sicherzustellen. Ist das Material vollständig aufgeschmolzen, können über Schwingquarzwaagen die einzelnen Verdampfungsraten gemessen und somit die Depositionsraten über den Heizstrom geregelt werden. Die Zeitkonstante dieser Regelung liegt im Bereich von unter einer Sekunde, Regelschwingungen sind typischerweise nach wenigen Sekunden abgeklungen.

Die Schwingquarzwaage besteht aus einem Quarzplättchen, das zu mechanischen Schwingungen mit seiner Eigenfrequenz (hier um $6 \mathrm{MHz}$ ) angeregt wird.

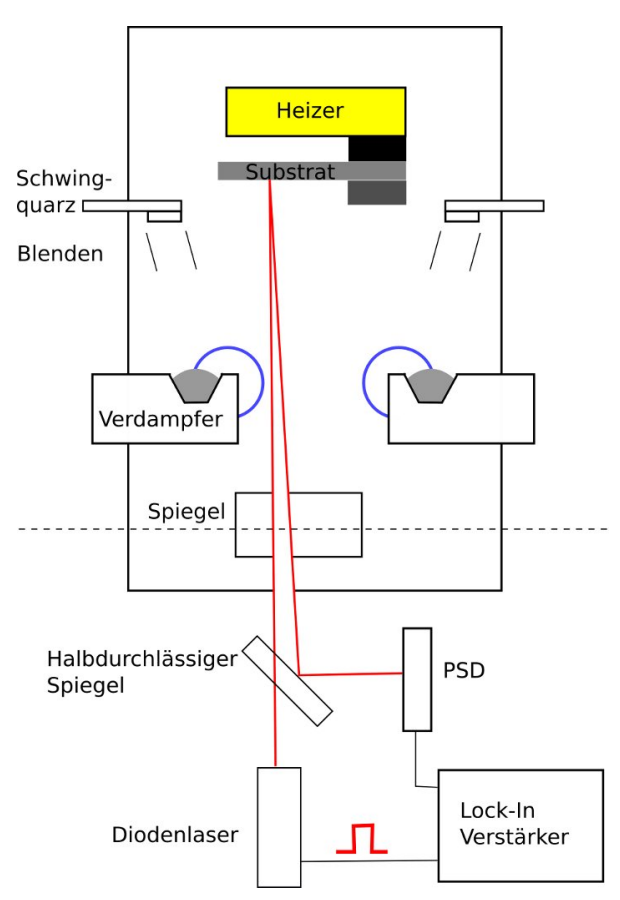

Abbildung 3.1: Skizze der Kammer zum Elektronenstrahlverdampfen mit optischem Aufbau zur Messung der Substratkrümmung. Durch das auf dem Quarz deponierte Material ändert sich die Eigenfrequenz des Oszillators. Bei bekannter akustischer Impedanz (Z-Ratio), Dichte und Molmasse lässt sich hieraus die Aufdampfrate bestimmen, die beim Elektronenstrahlverdampfen üblicherweise zwischen $0,3 \AA /$ sec und $20 \AA /$ sec liegt.

Da die Schwingquarzwaagen so über den einzelnen Verdampfern platziert sind, dass sie das Substrat nicht abschatten und gleichzeitig mit Blenden sichergestellt werden muss, dass die anderen Verdampfer nicht ebenfalls Material auf dem Schwingquarz deponieren, ist die Rate auf dem Schwingquarz und auf dem Substrat wegen der inhomogenen Verdampfungscharakteristik nicht identisch. Das Verhältnis der beiden (der so genannte Tooling-Faktor)

\footnotetext{
${ }^{1}$ Eisen, Reinheit: 99,95 \%, Quelle: Kurt J. Lesker, Palladium, Reinheit: 99,95 \% Quelle: Kurt J. Lesker
} 


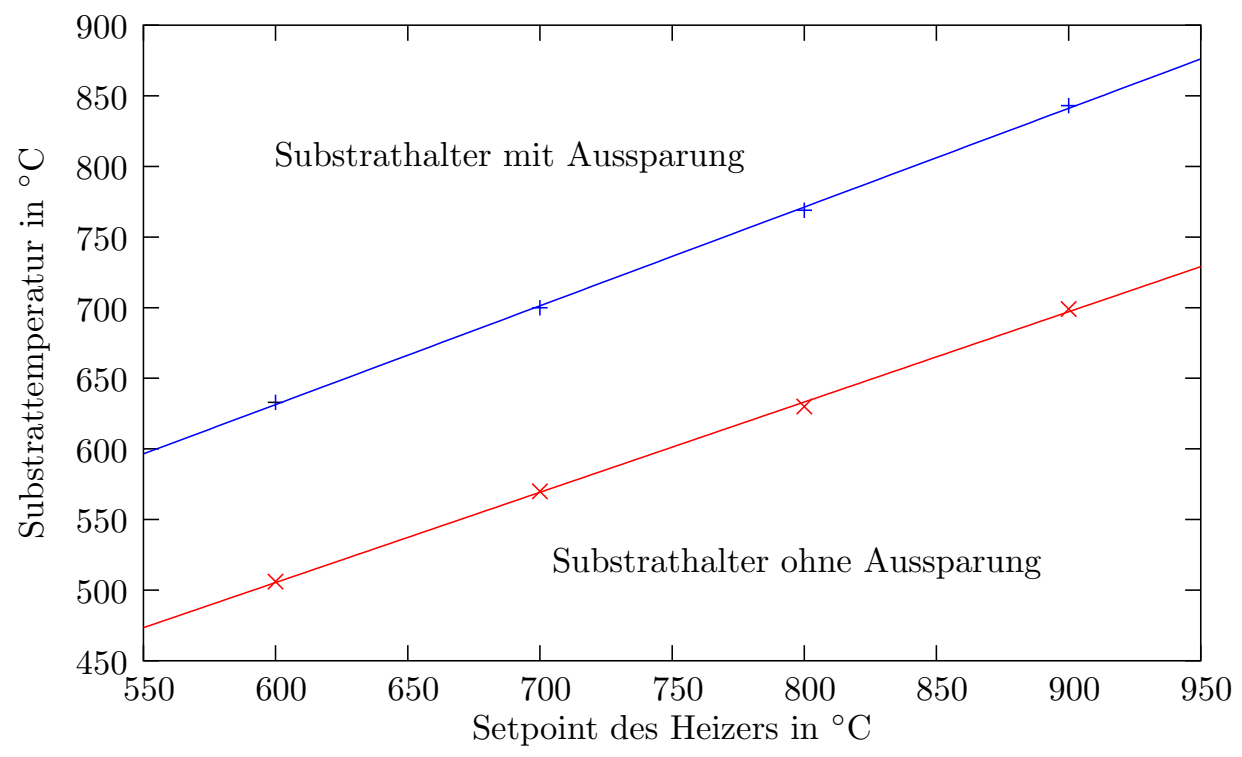

Abbildung 3.2: Kalibrierung des Substratheizers.

kann jedoch leicht bestimmt werden, indem die Dicke einer einkomponentigen Schicht zum Beispiel mit Kleinwinkel-Röntgenstreuung vermessen wird, und diese Dicke mit dem von der Schwingquarzwaage angezeigten Wert verglichen wird.

Als Substrate kamen sowohl thermisch oxidierte Siliziumwafer mit einer Oxidschicht von etwa $500 \mathrm{~nm}$ zum Einsatz (die natürliche Oxidschicht von wenigen Nanometern verhindert bei erhöhten Temperaturen nicht die Bildung von Palladiumsilizid), als auch einkristalline Magnesiumoxid-(100)Substrate. Eine neu konstruierte Strahlungsheizung, bestehend aus einer Wolframwendel, die hinter dem Substrathalter mäanderförmig in einen Stenanblock eingelegt ist, ermöglicht die Schichtdeposition bei erhöhten Temperaturen. In diesem Stenanblock befindet sich ein Thermoelement, das genau wie die Probe durch thermische Strahlung an den Heizer angekoppelt ist. Über die Temperatur dieses Thermoelements kann über einen Eurotherm-Regler ${ }^{2}$ die Heizleistung geregelt werden. Da dieser Regler lediglich eine vorgegebene Temperatur einregeln kann, wird mit Hilfe eines LabView-Programms regelmäßig dieser Setpoint erhöht, um die Möglichkeit zu erhalten, Temperaturrampen zu fahren. Je nach Depositionstemperatur kommen verschiedene Substrathalter zum Einsatz: Bei Raumtemperatur werden einfache Halter aus Edelstahl verwendet, für Depositionstemperaturen über $500^{\circ} \mathrm{C}$ wurden zwei

\footnotetext{
${ }^{2}$ Eurotherm Communicating Controller Type 825
} 
Halter aus Molybdän gefertigt, von denen der Halter für die Spannungsmessungen eine zusätzliche Aussparung unterhalb der Position des Substrats hat, um das Substrat direkt durch die Strahlung der Wolframwendel zu heizen. Um die Substrattemperatur verlässlich zu bestimmen, wurde zur Kalibrierung an der Einklemmung des Substrats auf der dem Heizer abgewandten Seite ein zusätzliches Thermoelement mit eingeklemmt, und so für beide Halter die Substrattemperatur bestimmt. Wie in Abbildung 3.2 ersichtlich, lässt sich für beide Probenhalter die Temperaturdifferenz zwischen Setpoint des Heizers und Substrattemperatur durch eine Gerade beschreiben, so dass im Folgenden immer direkt die tatsächliche Substrattemperatur angegeben wird.

\subsubsection{Gepulste Laserdeposition}

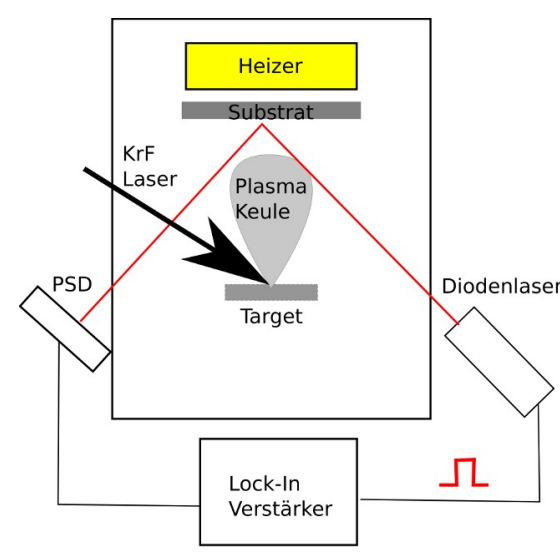

Abbildung 3.3: Skizze der deposition mit optischem Aufbau zur Messung der Substratkrümmung. Kammer zur gepulsten Laser-

Am Leibniz-Institut für Festkörperund Werkstoffforschung Dresden wurden während eines Aufenthalts dort in $\mathrm{Zu}-$ sammenarbeit mit Jörg Buschbeck einige Proben mit dem Verfahren der gepulsten Laserdeposition (PLD) hergestellt, die in ihren Eigenschaften deutlich von aufgedampften Schichten verschieden sind. Bei diesem in Abbildung 3.3 dargestellten Verfahren wird ein Target des zu deponierenden Materials mit einem sehr stark fokussierten KrF-Excimerlaser mit einer Wellenlänge von $248 \mathrm{~nm}$ mit Pulsen von 25 ns Dauer beschossen. Hierbei wird kurzzeitig einem sehr kleinen Bereich des Targets deutlich mehr thermische Energie zugeführt, als durch Wärmeleitung in das Volumen des Targets abgeführt werden kann. Das Material erhitzt lokal so stark, dass zu Beginn eines Pulses ein Teil der heißen Atome abdampft. Diese Materialwolke absorbiert dann während der restlichen Pulsdauer den größten Teil der einfallenden Strahlung und wird durch die hohe Energiedichte zu einem Plasma ionisiert. Diese Plasmakeule trifft nun mit hoher kinetischer Energie (bis zu $100 \mathrm{eV}$ [74]) auf das Substrat und schlägt sich dort nieder.

Im Gegensatz zum Verdampfen gibt es bei der gepulsten Laserdeposition zwei verschiedene Möglichkeiten, Legierungen herzustellen: Zum einen ist es möglich, ein Legierungstarget einzusetzen, das dann stöchiometrisch auf das Substrat abgeschieden wird, zum anderen ist es aber auch möglich, verschie- 
dene elementare Targets zu verwenden und die Zusammensetzung der Schicht durch die Zahl abwechselnder Laserpulse auf die jeweiligen Elementtargets einzustellen. Durch die hohe kinetische Energie ist eine Durchmischung der obersten Atomlagen mit den neu deponierten Atomen gewährleistet [75], so dass die Homogenität der abgeschiedenen Schichten sichergestellt ist.

\subsubsection{Aufdampfen mit Ionenstrahlunterstützung}

Um die für die Phasen- und Strukturbildung relevanten Prozesse während des Schichtwachstum zu beeinflussen, ist es möglich das Substrat, auf dem der Film abgeschieden wird, zusätzlich mit niederenergetischen Ionen zu bestrahlen. Dieses in Abbildung 3.4 dargestellte Verfahren wird als Ion Beam Assisted Deposition (IBAD) bezeichnet. Im Rahmen dieser Arbeit wurde eine mit Argon ${ }^{3}$ betriebene Ionenquelle ${ }^{4}$ verwendet, die bei einer Ionenenergie von 20 bis $110 \mathrm{eV}$ Ionenströme im Bereich von $20 \mathrm{~mA}$ bis $\mathrm{zu}$ $150 \mathrm{~mA}$ erzeugen kann. Zur Erzeugung dieses Ionenstroms wird an der Kanone ein Plasma gezündet, und der Gaszufluss des zu beschleunigenden Gases so geregelt, dass in dem Plasma ein konstanter Strom von bis zu einem Ampère fließt. Nimmt man an, dass jedes Gasatom nur einmal ionisiert wird, erhält man damit einen Gasstrom von $10^{19}$ Gasatomen bzw. bis zu $10 \mathrm{~cm}^{3}$ Gas bei Normaldruck pro Sekunde. Diese Gaslast ist so hoch, dass auch bei der Verwendung mehrerer Turbomolekularpumpen der Druck in der Depositionskammer bis auf $10^{-4}$ mbar ansteigt. Bei diesem Druck ist das Restgas in der Kammer so leitfähig, dass die Verwendung von Elektronenstrahlverdampfern unmöglich ist, die notwendigen Spannungen von bis zu $10 \mathrm{kV}$ lassen sich nicht gegen Überschläge durch das Restgas isolieren. Deshalb wurde die Herstellung dieser Proben in einer anderen Vakuumanlage (beschrieben in [76]) vorgenommen, die mit thermischen Verdampfern (Knudsenzellen) ausgestattet ist. Diese Zellen bestehen aus einem

\footnotetext{
${ }^{3}$ Argon, Reinheit: 99,9999 \% Quelle: Air Liquide

${ }^{4}$ Commonwealth Scientific Mark I Griddless Ion Source
} 
konusförmigen Keramiktiegel, in dem sich das zu verdampfende Material ${ }^{5}$ befindet und der von außen durch die Strahlung einer Wolframwendel geheizt wird. Dieser Heizer ist außen zunächst von mehreren Strahlungsschilden aus Molybdän und Tantal umgeben und wird komplett von einem wassergekühlten Mantel abgeschirmt.

Bei diesen Verdampfern kann nicht direkt die Rate vorgegeben werden, sondern nur die Zellentemperatur, die typischerweise 50 bis 100 Grad unter der Schmelztemperatur des zu verdampfenden Materials liegt. In diesem Bereich sublimiert bereits ein geringer Teil des Materials, so dass typische Depositionsraten im Bereich von unter einem Ångstrom pro Sekunde liegen. Würde die Temperatur der Zelle bis zur Schmelztemperatur erhöht, hätte dies zwar eine deutliche Steigerung der Depositionsrate zur Folge, allerdings besteht insbesondere beim Erstarren des Materials eine große Gefahr, dass das Material den Keramiktiegel sprengt. Um thermische Verspannungen des Tiegels zu minimieren, ist es ebenfalls notwendig die Temperatur der Zelle nur sehr langsam zu verändern. Auch deshalb ist eine schnelle aktive Regelung der Verdampfungsrate wie beim Elektronenstrahlverdampfen nicht möglich.

Wird nun während des Schichtwachstums das Substrat gleichzeitig mit Ionen bestrahlt, hängt die tatsächliche Depositionsrate außer von der Tiegeltemperatur auch stark von dem Gasdruck in der Kammer und der gewählten Ionenenergie ab. Daher wurden zunächst die Verdampfer auf Betriebstemperatur gefahren, die Ionenkanone gezündet und eine reine Eisenschicht, eine reine Palladiumschicht, und schließlich eine Eisen-Palladiumschicht aufgedampft. Aus der Depositionszeit und der mittels Kleinwinkelröntgen gemessenen Schichtdicke der elementaren Schichten lassen sich die Depositionsraten für die jeweiligen Bedingungen genau bestimmen, woraus dann die $\mathrm{Zu}-$ sammensetzung der Eisen-Palladiumschicht mit hinreichender Genauigkeit bestimmt werden kann.

Auch in dieser Kammer ist es möglich, das Substrat während der Deposition zu heizen. Auch hier wurde analog zur Kalibrierung beim Elektronenstrahlverdampfen mit einem zweiten Thermoelement die tatsächliche Temperatur des Substrats bestimmt.

\subsection{Aufbau zur Messung mechanischer Span- nungen}

Um den Spannungszustand eines epitaktischen Films während des Wachstums messen zu können, wurde sowohl beim Elektronenstrahlverdampfen als auch bei der PLD mit einem optischen Aufbau die Änderung der Substratkrümmung vermessen. Hierzu befindet sich im unteren Teil der Kammer zum Elektronenstrahlverdampfen ein Spiegel, über den ein Laserstrahl von einem

\footnotetext{
${ }^{5}$ Eisen, Reinheit: 99,95 \%, Quelle: Kurt J. Lesker, Palladium, Reinheit: 99,95 \%, Quelle: Heraeus
} 
optischen Tisch aus zum Substrat gelenkt und die Position des reflektierten Strahls vermessen wird. Ein Schema des Aufbaus ist in Abbildung 3.1 gezeigt.

Für die Spannungsmessungen wurden MgO-Einkristalle mit einer Dicke von $0,15 \mathrm{~mm}$ und einer Fläche von $5 \mathrm{~mm} \times 20 \mathrm{~mm}$ als Substrate verwendet. Für die Messungen wurden sie mit einer der kurzen Kanten zwischen zwei Molybdänklötze geklemmt, von denen der Untere neben der Aussparung auf dem Probenhalter aufliegt. Diese Einspannung wird von zwei Schrauben aus Tantal auf den eigentlichen Probenhalter gepresst. Um trotz der geringen Kontaktfläche zwischen Halter und Substrat die Substrattemperatur schnell und gleichmäßig zu erreichen, wurde aus dem Halter die bereits erwähnte Aussparung gefräst, durch die die Strahlung der Heizwendel direkt das Substrat erreicht.

Die Position des reflektierten Laserstrahls wird mit einem positionssensitiven Detektor (PSD) gemessen. In diesem Detektor wird ein Halbleitermaterial durch den Einfall von Licht leitfähig, wodurch zwischen je zwei verschiedenen Kontakten an der Vorder- und Rückseite des Sensors Ströme fließen können, deren Stärke von der Lichtintensität und dem Auftreffpunkt abhängen.

Um die Signalqualität zu verbessern, werden zunächst in einem Vorverstärker die Summe und die Differenz der beiden Teilströme gebildet, und beide jeweils um einen konstanten Faktor verstärkt. Das Summensignal ist nun proportional zur Intensität des auf den Detektor fallenden Lichts, während das Differenzsignal sowohl zur Gesamtintensität als auch zur relativen Position des Lichtpunkts auf dem Detektor proportional ist. Damit ist der Quotient aus Summen- und Differenzsignal intensitätsunabhängig, und hängt nur vom Auftreffort des Lichts auf den Detektor ab.

Da über den Spiegel in der Kammer nicht nur der Laserstrahl, sondern auch das Streulicht der Verdampfer auf den PSD fällt, wird das Signal-zuRauschverhältnis durch einen Lock-In-Verstärker erhöht. Hierfür wird einerseits die Intensität der Laserdiode mit einem rechteckigen Referenzsignal (Frequenz $117 \mathrm{~Hz}$ ) moduliert, andererseits die Ausgangssignale des Vorverstärkers mit einem symmetrischen Rechtecksignal zwischen -1 und +1 der selben Frequenz und Phase multipliziert. Der Verstärker integriert nun immer über dieses Produkt, so dass sich alle Signalbestandteile, die nicht die Periodizität des Referenzsignals besitzen, wegheben. Trotz dieser Methode erzeugt die Endstufe des Verstärkers einen leichten Offset, so dass der Wert, der bei geschlossenem Shutter direkt vor der Deposition aufgenommen wurde, von allen Messpunkten subtrahiert wurde. Zur Kalibrierung des Messaufbaus lässt sich der PSD mit einer Mikrometerschraube verschieben, so dass hieraus der Faktor zwischen der Ortsänderung und der Änderung des korrigierten Detektorsignals bestimmt werden kann. 


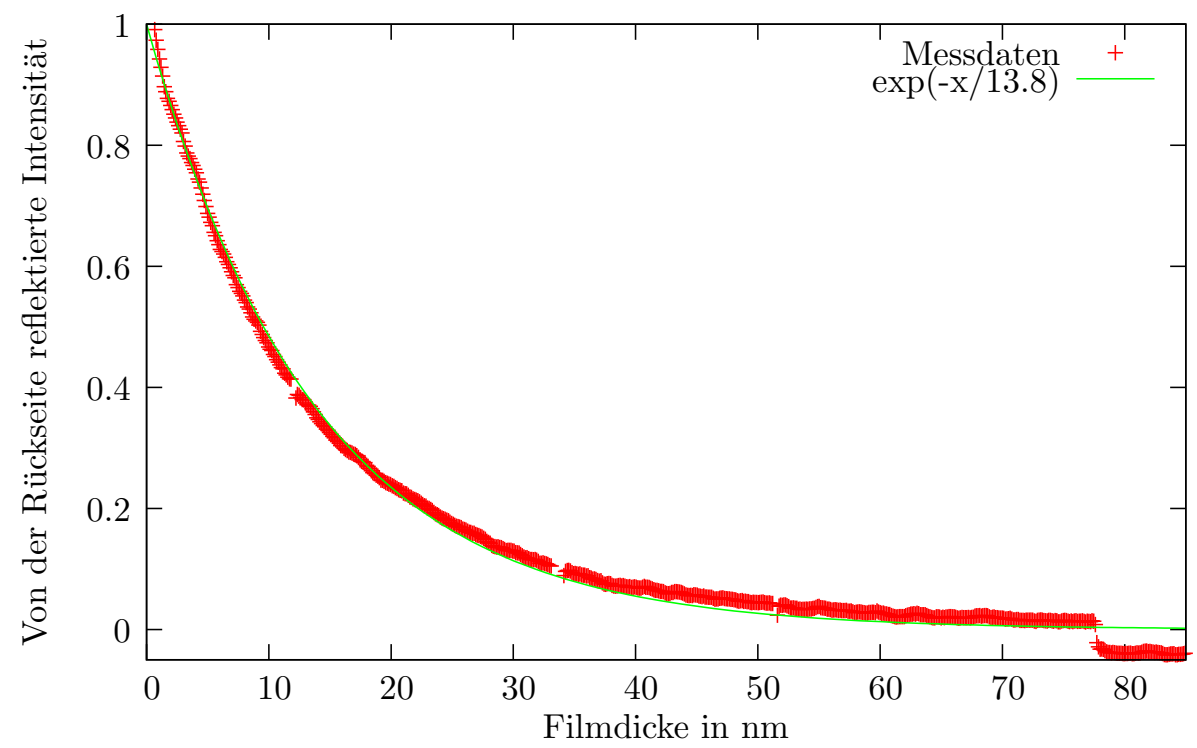

Abbildung 3.5: Exponentieller Zusammenhang zwischen der Schichtdicke und der Transmission, nach $77.5 \mathrm{~nm}$ wurde der Laserstrahl unterbrochen.

Aus den zur Bestimmung der Substratkrümmung aufgenommenen Daten lässt sich ein Maß für die Rauigkeit der Filmoberfläche gewinnen. Hierzu muss nur das Summensignal des PSDs betrachtet werden, welches zur Intensität des auf den PSD fallenden Lichts proportional ist. Für jede Grenzfläche gilt, dass die Summe aus transmittierter und reflektierter Intensität konstant ist. Dies kann man sich zunutze machen, um die Änderung der Reflektivität zu bestimmen. Außer dem bisher betrachteten Laserstrahl, der an der aufgedampften Schicht reflektiert wird, tritt auch ein Teil des Strahls in das Substrat ein. Von diesem Anteil wird wiederum ein gewisser Teil an der hinteren Grenzfläche des Substrats reflektiert und tritt aus der Vorderseite wieder aus. Durch den langen Strahlengang bis zum Austrittsfenster aus der Anlage und den nie vollständig senkrechten Einfall ist dieser an der Rückseite des Substrats reflektierte Strahl einige Zentimeter gegen den an der Vorderseite des Substrats (also am Film) versetzt. Somit ist sichergestellt, dass während der Deposition immer nur einer der Strahlen auf den Detektor fällt. Die Intensität des Strahls von der Rückseite des Substrats ist somit ein Maß dafür, wie sich das Verhältnis zwischen dem insgesamt (also diffus und spekular) reflektierten und transmittierten Strahl durch die Metallschicht im Lauf der Deposition verändert, und wird in Abbildung 3.5 sehr gut durch einen exponentiellen Abfall beschrieben.

Der PSD registriert jedoch nicht die gesamte vom Film reflektierte In- 
tensität, sondern nur den spekular gestreuten Anteil. Je nach Rauigkeit des Films wird jedoch ein Teil der Intensität auch diffus gestreut, so dass eine Abweichung von einer exponentiellen Zunahme der Intensität als Änderung der Oberflächenrauigkeit interpretiert werden kann.

Da alle auftretenden Winkel sehr klein sind (unter 0,1 Grad), lassen sich die auftretenden trigonometrischen Funktionen linearisieren, so dass sich ein einfacher Zusammenhang zwischen der Positionsänderung $\Delta x$ des reflektierten Laserstrahls, der Länge des Abstands Substrat-Detektor L, des Abstands des Auftreffpunkts des Laserstrahls von der Einspannung des Substrats $a$ und dem Krümmungsradius $R$ des Substrats ergibt [77]:

$$
\frac{1}{R}=\frac{\Delta x}{2 a L}
$$

Über die Stoney-Gleichung [78] lässt sich nun aus der Krümmung des Substrats die über die Schichtdicke gemittelte Spannung berechnen:

$$
\frac{F}{w}=\langle\sigma\rangle d=\frac{1}{6} \frac{E_{s}}{1-\nu_{s}} s^{2} \frac{1}{R}
$$

Das Produkt aus mittlerer Spannung $\sigma$ und Schichtdicke $d$ - was Kraft $F$ pro Einheitsbreite $w$ entspricht - lässt sich also über die elastischen Eigenschaften (Elastizitätsmodul $E_{s}$ und Querkontraktionszahl $\nu_{s}$ ) des Substrats und dessen Dicke $s$ aus der Krümmung des Substrats bestimmen.

Innerhalb dieser Arbeit wird die Konvention verwendet, für Zugspannungen das positive Vorzeichen zu verwenden. Solche Zugspannungen führen zu einer positiven Längenänderung und entstehen zum Beispiel in einem Film, der epitaktisch auf einem Substrat aufwächst, das eine größere Gitterkonstante als der Film hat, und führen zu einer konkaven Krümmung des Substrats.

Auch an den mit PLD hergestellten Schichten wurde mit einem entsprechenden optischen Aufbau die Krümmung des Substrates während der Deposition gemessen, um die mechanischen Spannungen im Film zu bestimmen. Hierbei war es wie in Abbildung 3.3 dargestellt, im Gegensatz zu der beim Aufdampfen verwendeten Geometrie nicht möglich, den Laserstrahl senkrecht zum Substrat zu führen. Stattdessen wurden zwei parallele Laserstrahlen mit einem Abstand von $11 \mathrm{~mm}$ verwendet, die durch ein Fenster in der Depositionskammer zum Substrat verliefen, diese unter einem Winkel von 45 Grad trafen, und dann durch ein anderes Fenster zum Detektor fielen. Die Verwendung von zwei Laserstrahlen und zwei Detektoren hat den Vorteil, dass statt der Bewegung eines Laserpunktes die Änderung des Abstands zwischen den beiden Punkten gemessen werden kann, um daraus die Krümmung zu bestimmen. Diese Methode hat den Vorteil, dass sich alle Bewegungen des gesamten Substrates, die beide Lichtpunkte gleich bewegen, herausheben. 
Die Berechnung der Substratkrümmung aus der Änderung des Abstands der Lichtpunkte erfolgt bei diesem schrägen Strahlengang völlig analog zum senkrechten Einfall, wie in Anhang A gezeigt wird.

\subsection{Tempern der Proben}

Da sich je nach Substrattemperatur verschiedene Kristallstrukturen des FePdFilms einstellen, ist unter Umständen eine thermische Nachbehandlung der Proben notwendig. Hierzu wurden die Proben in ein an einem Ende verschlossenes Quarzrohr eingebracht und an eine Vakuumpumpe angeschlossen. Zunächst wurde das Quarzrohr nur grob auf einen Druck unter 1 mbar abgepumpt und wieder mit Argon ${ }^{6}$ geflutet, danach auf einen Druck von unter $3 \cdot 10^{-5}$ mbar evakuiert, und noch mindestens zwei weitere Male mit Argon gespült und evakuiert. Zuletzt wurde das Quarzrohr mit einen Druck von 600 mbar Argon befüllt und verschweißt. Eine so verschlossene Ampulle kann nun an einem Draht in einen Rohrofen gehängt werden, und nach Ablauf der Temperzeit entweder an diesem Draht wieder aus dem Ofen gezogen werden, oder der Draht wird gekappt, und die Ampulle fällt in einen Wassereimer, der unter dem Rohrofen platziert werden kann, um ein schnelleres Abschrecken zu erreichen. Die hierbei zu erreichende Abkühlrate lässt sich auf über $100 \mathrm{~K} / \mathrm{s}$ abschätzen, da die Proben innerhalb von wenigen Sekunden nicht mehr glühen.

\subsection{Präparation freistehender Filme}

Zur Untersuchung der intrinsischen Eigenschaften dünner Filme ist es entscheidend, dass diese Filme nicht durch ein hartes Substrat in ihrer Verformung eingeschränkt werden. Hierbei existieren verschiedene Möglichkeiten: Bei NiMnGa-Filmen ist es zum Beispiel möglich, wasserlösliche Substrate wie NaCl-Einkristalle zu verwenden [80], chemisch selektiv ätzbare Zwischenschichten wie Chrom zwischen Film und Substrat aufzubringen [81], oder die Filme mechanisch vom Substrat abzureißen [82]. Jede dieser Methoden hat gewisse Nachteile: Beim Abreißen wird der Film mechanisch stark verformt, was die Eigenschaften des Films massiv verändern kann. Opferschichten und Ionenkristalle unter dem Film schränken durch ihren Schmelzpunkt bzw. eine erhöhte Diffusion in den Film den für die Deposition zur Verfügung stehenden Temperaturbereich stark ein. Damit lassen sie sich nicht direkt auf das bei mittleren Temperaturen entmischende System Eisen-Palladium übertragen.

\footnotetext{
${ }^{6}$ Argon, Reinheit: 99,999 \%, Quelle: Air Liquide
} 

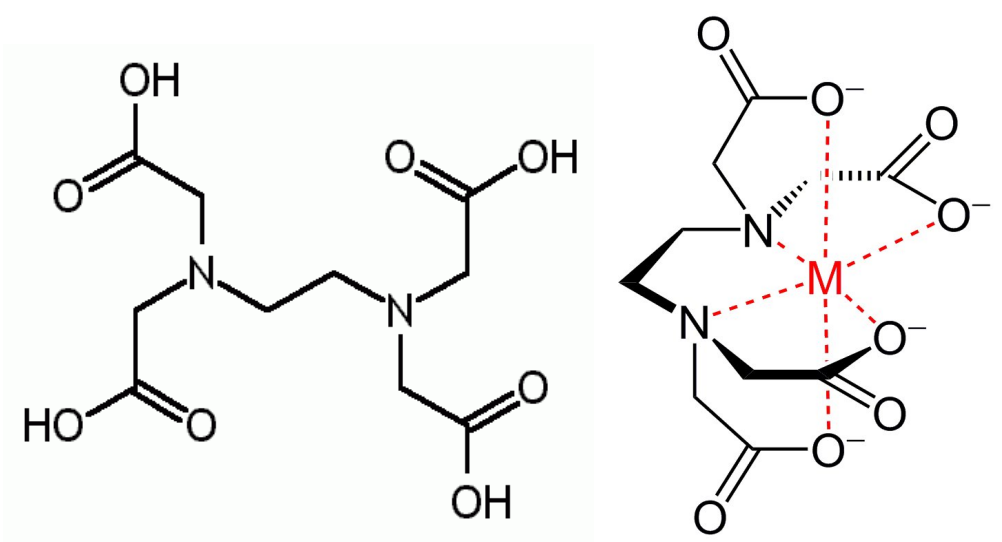

Abbildung 3.6: Links: Strukturformel eines EDTA-Moleküls. Rechts: Stereographische Projektion eines Metall-EDTA-Komplexes. Beide aus [79].

Daher wurden im Rahmen dieser Arbeit zwei nasschemische Verfahren entwickelt, um Eisen-Palladium-Filme direkt von MgO-Substraten abzulösen.

Beim ersten Verfahren wird eine wässrige Lösung von Ethylendiamintetraessigsäure (EDTA), bzw. des Natriumsalzes dieser Säure (Na-EDTA) angesetzt. In dieser Lösung bilden die im Wasser gelösten Magnesium-Ionen einen Chelatkomplex, in dem das Magnesiumion so gebunden wird, so dass es für weitere Reaktionen nicht mehr zur Verfügung steht. Wie in Abbildung 3.7 deutlich zu sehen, hat neben der Temperatur auch der pH-Wert der Lösung einen großen Einfluss auf die Rate, mit der die MgO-Substrate dünner werden. Dieser wurde durch Zugabe von Salzsäure bzw. NatriumhydroxidLösung eingestellt.

Aufnahmen mit einem Lichtmikroskop (Abbildung 3.8) zeigen außerdem deutlich, dass nicht nur die Oberfläche mit Ätzgruben übersät ist, sondern auch längliche Gräben aufweist, die entlang der [110]-Richtungen des Substrats verlaufen.

In EDTA lösen sich auch $\mathrm{Fe}^{++}$-Ionen sehr gut. Dies hat zur Folge, dass sich Schichten, die Eisenausscheidungen an der Grenzfläche zum Substrat haben, innerhalb eines Tages ablösen lassen, da hier die Kontaktfläche zum Substrat deutlich verringert wird, in dem die Ausscheidungen sich auflösen. Auch an anderen Proben ist zu beobachten, dass sich der Eisengehalt leicht verringert, was auf eine Verarmung der Oberflächen an Eisen zurückzuführen ist. Eine konzentrierte Lösung von Natriumhydorgencarbonat $\left(\mathrm{NaHCO}_{3}\right)$ löst ebenfalls $\mathrm{MgO}$, wenn auch mit einer deutlich geringeren Geschwindigkeit von nur ca. $80 \mathrm{~nm}$ pro Stunde. Hierbei lässt sich die Reaktion durch Tempera- 


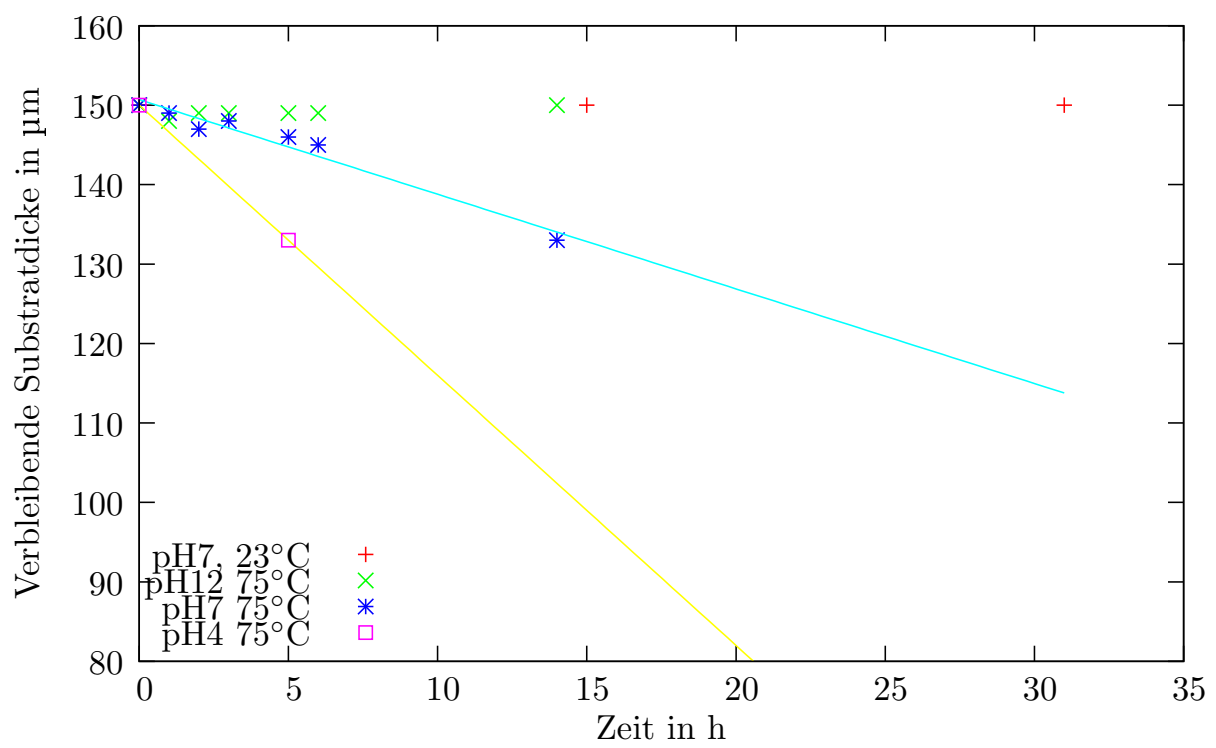

Abbildung 3.7: Ätzrate von $\mathrm{MgO}$ in 0,3-molaren EDTA-Lösungen.

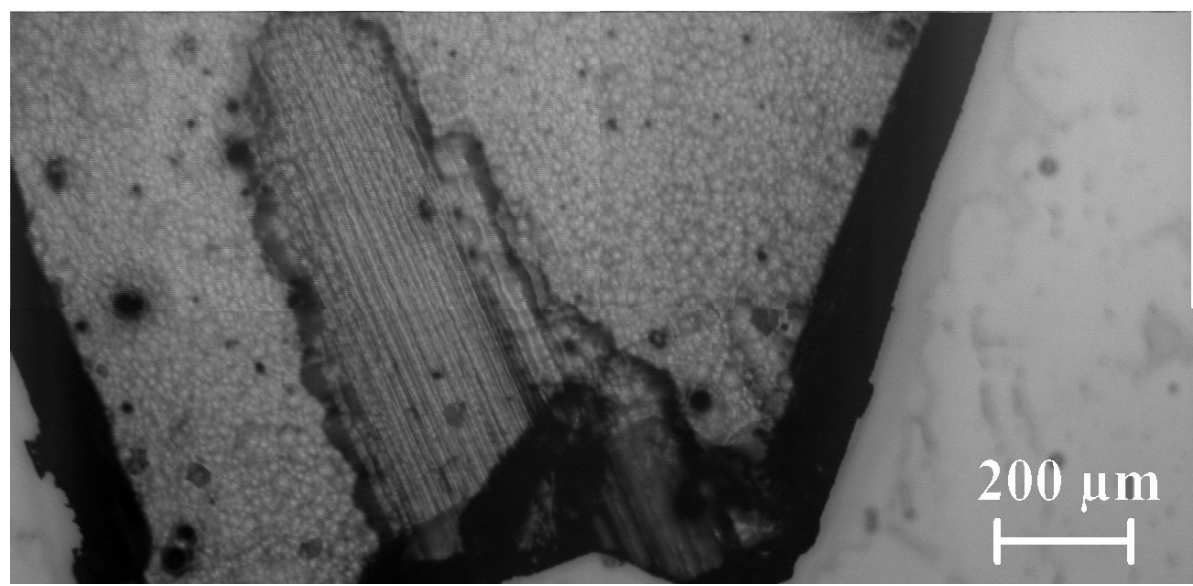

Abbildung 3.8: Lichtmikroskopische Aufnahme eines angeätzten $\mathrm{MgO}$ Substrats. 
turerhöhung nicht wesentlich beschleunigen, da sich das Natriumhydorgencarbonat schon bei geringen Temperaturen zu Natruimcarbonat, Wasser und Kohlendioxid zersetzt.

\subsection{Röntgenstreumethoden}

Durch Röntgenstreumethoden lassen sich nicht nur viele Informationen über die Struktur der Schichten gewinnen, durch Kleinwinkelstreuung ist es auch möglich, die Dicke von Schichten im Bereich zwischen $100 \AA$ und $1000 \AA \mathrm{zu}$ bestimmen. Hierfür wird bei streifendem Einfall die Abhängigkeit der Intensität der gestreuten Röntgenstrahlung vom Winkel zwischen Substrat und einfallendem Strahl bestimmt. Unterhalb eines kritischen Winkels (der nur vom Brechungsindex des Materials abhängt) kommt es zur Totalreflexion. Nachdem dieser Winkel überschritten ist, fällt die Intensität nicht monoton ab, sondern es kann ein Muster aus Maxima und Minima beobachtet werden, das sich als Interferenz des an der Grenzfläche Luft-Probe reflektierten Strahls mit dem an der Grenzfläche Schicht-Substrat reflektierten Strahl verstehen lässt. Die Lage der Extrema hängt außer vom Brechungsindex des Materials nur von der Schichtdicke ab, während die Einhüllende von dem Absorptionsvermögen der Probe und der Rauigkeit der Grenzflächen bestimmt ist. Die Auswertung der Daten erfolgte mit Hilfe des Programms parratt ${ }^{7}$, das mit Hilfe des Parratt-Algorithmus [83] sowohl die Schichtdicken als auch die Rauigkeiten durch iterative Anpassung bestimmt. Eine exemplarische Messung und Simulation ist in Abbildung 3.9 gezeigt.

Bei größeren Streuwinkeln lassen sich aus Röntgenstreuexperimenten detaillierte Informationen über die Kristallstruktur gewinnen: Die Bragg-Bedingung verknüpft die Winkel $\Theta$, bei denen für eine bestimmte Wellenlänge $\lambda$ ein Maximum der gestreuten Intensität beobachtet wird mit den Abständen $d$ der streuenden Atomebenen:

$$
2 d \sin (\Theta)=n \lambda
$$

Für orthorhombische Kristallstrukturen mit den Gitterparametern $a, b, c$ (insbesondere für die Spezialfälle des kubischen Gitters mit $a=b=c$ und des tetragonalen Gitters mit $a=b$ ) gilt für den Abstand zweier Netzebenen mit den Millerschen Indizes $(h k l)$ :

$$
d_{h k l}=\frac{1}{\sqrt{\left(\frac{h}{a}\right)^{2}+\left(\frac{k}{b}\right)^{2}+\left(\frac{l}{c}\right)^{2}}}
$$

${ }^{7}$ (C) 1997-99, Christian Braun, HMI Berlin 


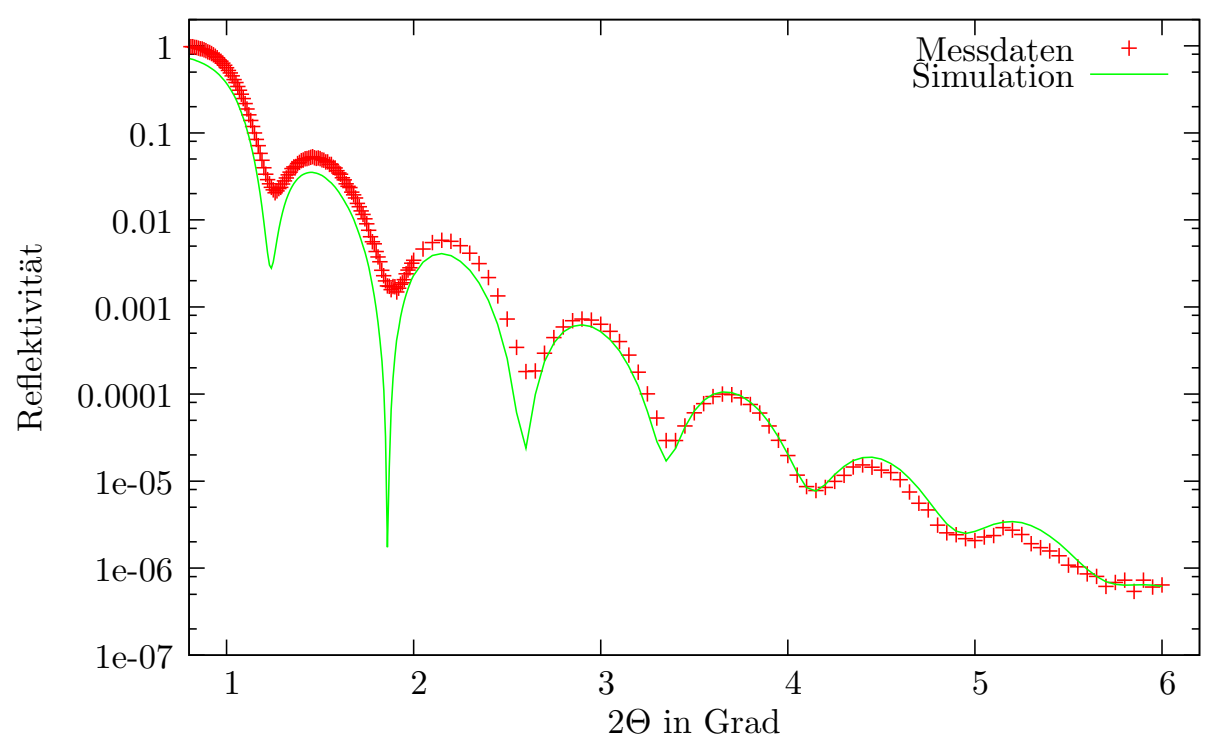

Abbildung 3.9: Kleinwinkelstreuungsmessung und Simulation einer Palladiumschicht auf $\mathrm{SiO}_{2}$. Verwendete Simulationsparameter: Dicke $110 \AA$, RMSRauigkeit der Schicht 6,8 A, RMS-Rauigkeit des Substrats 4,8 A.

Für eine vorgegebene Struktur lassen sich nun die auftretenden Ebenenabstände und damit die zu erwartenden Reflexe bestimmen. Für die wichtigsten in dieser Arbeit auftretenden Phasen sind in Tabelle 3.1 die Gitterstrukturen und Gitterparameter und in Tabelle 3.2 die Lagen der Peaks für die $K_{\alpha}$-Strahlung von Kupfer $(\lambda=1,5413 \AA)$ dargestellt. Mit Hilfe der Software PowderCell [84] ist es auch möglich, die angenommene Struktur so zu variieren, dass die von dieser Struktur erzeugten Reflexe möglichst gut zu einem gemessenen Diffraktogramm passen.

Die in dieser Arbeit vorgestellten $\Theta-2 \Theta$ Messungen wurden zum größten Teil mit einem Bruker-Diffraktometer ${ }^{8}$ gemessen. Für Messungen im Bereich zwischen $-15^{\circ} \mathrm{C}$ und $140^{\circ} \mathrm{C}$ kam ein Siemens-Diffraktometer ${ }^{9}$ zum Einsatz, für das im Rahmen dieser Arbeit ein Probenhalter, dessen Temperatur über ein Peltierelement gesteuert werden kann, konstruiert wurde. Dieser in Abbildung 3.10 dargestellte Probenhalter besteht zunächst aus einer wasserkühlbaren Kupfer-Basisplatte, auf der das Peltierelement aufliegt. Auf dessen Oberseite befindet sich ein Aluminiumblock für die Wärmeleitung zwischen Peltierelement und Probe. Um die Röntgenstreuung an diesem Aluminiumblock zu minimieren, liegt die Probe nur auf einer schmalen Säule auf, der

\footnotetext{
${ }^{8}$ Bruker D8 Advance

${ }^{9}$ Siemens D5000
} 


\begin{tabular}{|l|c|l|c|}
\hline Material & Struktur & Gitterkonstante $a, c$ & Quelle \\
\hline $\mathrm{Fe}$ & $\mathrm{bcc}$ & $2,879 \AA$ & {$[85]$} \\
$\mathrm{Pd}$ & fcc & $3,89 \AA$ & {$[85]$} \\
$\mathrm{MgO}$ & $\mathrm{NaCl}$ & $4,21 \AA$ & {$[85]$} \\
\hline $\mathrm{Fe}_{80} \mathrm{Pd}_{20}$ & $\mathrm{bcc}$ & $2,960 \AA$ & {$[35]$} \\
$\mathrm{Fe}_{70} \mathrm{Pd}_{30}$ & fcc & $3,752 \AA$ & {$[18]$} \\
$\mathrm{Fe}_{70} \mathrm{Pd}_{30}$ & fct & $3,832 \AA, 3,657 \AA$ & {$[35]$} \\
$\mathrm{Fe}_{60} \mathrm{Pd}_{40}$ & fct & $3,826 \AA, 3,699 \AA$ & {$[35]$} \\
$\mathrm{Fe}_{50} \mathrm{Pd}_{50}$ & $\mathrm{~L} 1_{0}$ & $3,855 \AA, 3,714 \AA$ & {$[85]$} \\
\hline
\end{tabular}

Tabelle 3.1: Zur Simulation verwendete Gitterkonstanten.

\begin{tabular}{|c|c|c|c|c|c|c|c|}
\hline \multicolumn{2}{|c|}{ Material } & $(h k l)$ & $2 \Theta$ & $(h k l)$ & $2 \Theta$ & $(h k l)$ & $2 \Theta$ \\
\hline $\mathrm{Pd}$ & fcc & $(111)$ & 40,255 & $(200)$ & 46,789 & $(220)$ & 68,323 \\
\hline $\mathrm{Fe}$ & $\mathrm{bcc}$ & $(110)$ & 44,779 & $(200)$ & 65,186 & $(211)$ & 82,559 \\
\hline $\mathrm{Fe}_{70} \mathrm{Pd}_{30}$ & bcc & $(110)$ & 43,497 & $(200)$ & 63,204 & $(211)$ & 79,850 \\
\hline $\mathrm{Fe}_{70} \mathrm{Pd}_{30}$ & fcc & $(111)$ & 41,660 & $(200)$ & 48,486 & $(220)$ & 70,998 \\
\hline $\mathrm{Fe}_{70} \mathrm{Pd}_{30}$ & fct & & & $(200)$ & 47,4116 & $(002)$ & 49,830 \\
\hline $\mathrm{Fe}_{50} \mathrm{Pd}_{50}$ & $\mathrm{~L} 1_{0}$ & $(100)$ & 23,053 & $(001)$ & 23,941 & $(110)$ & 32,479 \\
\hline & & $(111)$ & 41,039 & $(200)$ & 47,111 & $(002)$ & 49,014 \\
\hline
\end{tabular}

Tabelle 3.2: Mit PowderCell [84] simulierte Peaklagen für Kupfer $\mathrm{K}_{\alpha^{-}}$ Strahlung der Wellenlänge 1,5413 Å. 


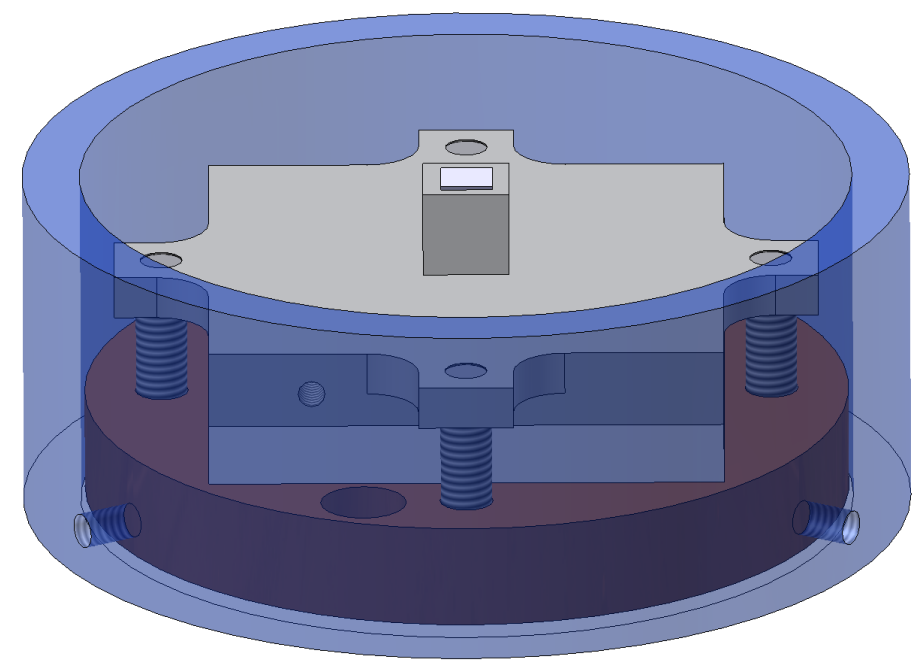

Abbildung 3.10: Skizze des neu konstruierten Probenhalters für Röntgenmessungen bei variablen Temperaturen: Das Peltierelement liegt zwischen der Basisplatte aus Kupfer und dem Probenträger aus Aluminium.

größte Teil liegt unter der Fokusebene. Um das Peltierelement nicht thermisch kurzzuschließen, sind die Schrauben zwischen Basisplatte und Aluminiumblock aus Kunststoff gefertigt. Die Probenhöhe wird durch die variable Aufhängung in einem Aluminiumrohr eingestellt. Um Kondenswasser auf der Probe zu vermeiden, wurde der Halter mit Silicagel-Perlen gefüllt, und eine mit Vakuumfett abgedichtete Kunststoff-Kappe mit Kapton-Fenstern für den ein- und den ausfallenden Strahl über den kompletten Probenhalter gestülpt.

Sind innerhalb der zu untersuchenden Probe viele regellos orientierte Kristallite vorhanden, liefert eine Messung, bei dem der Winkel zwischen Quelle und Probenoberfläche und gleichzeitig damit auch der Winkel zwischen Probenoberfläche und Detektor jeweils identisch ist (daher auch $\Theta-2 \Theta$ Scan genannt) alle Informationen zur Kristallstruktur. Wenn nun die Kristallite einer Probe nicht regellos verteilt sind, sondern eine bevorzugte Orientierung besitzen, kann es sein, dass manche Reflexe in einen $\Theta-2 \Theta$ Scan nicht auftreten. Eine Polfigurmessung liefert dagegen Informationen über die Verteilung der Kristallite: Hierzu werden $\Theta$ und $2 \Theta$ fest auf den entsprechenden Wert einer Ebenenschar eingestellt, und dann die Probe um die anderen beiden Achsen des 4-Kreis-Goniometers gedreht, wie in Abbildung 3.11 dargestellt.

Röntgenmessungen bei tieferen Temperaturen wurden in Zusammenar- 


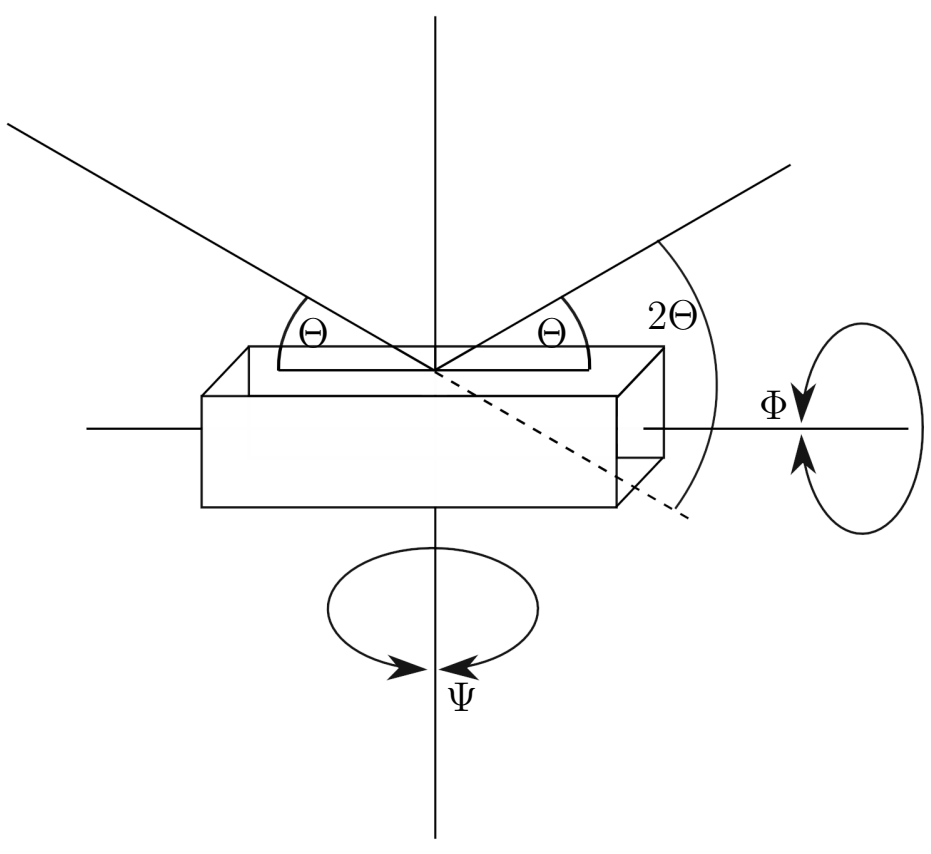

Abbildung 3.11: Bezeichnungen der Drehachsen eines 4-Kreis Goniometers.

beit mit dem Institut für Kristallographie am Geowissenschaftlichen Zentrum Göttingen, bzw. in Zusammenarbeit mit dem Lehrstuhl Werkstoffe der Mikrotechnik am Institut für Werkstoffe der Fakultät für Maschinenbau an der Ruhr-Universität Bochum durchgeführt, die Texturmessungen wurden am X'Pert des Instituts für Materialphysik in Göttingen durchgeführt. Weitere Röntgenmessungen an den mittels gepulster Laserdeposition hergestellten Schichten wurden von Jörg Buschbeck am Leibniz-Institut für Festkörperund Werkstoffforschung Dresden durchgeführt. Hierbei wurde die sogenannte $\sin ^{2} \Phi$-Methode [86] verwendet, bei der in einem 4-Kreis Goniometer verschiedene Reflexe vermessen werden und daraus die Gitterkonstante in Abhängigkeit des Winkels $\Phi$ zwischen der Strahlebene und der Probennormalen bestimmt wird. Trägt man nun die Gitterkonstanten gegen $\sin ^{2} \Phi$ auf, erhält man einen linearen Zusammenhang, an dem man für kubische Gitterstrukturen die Dehnung der Zelle in der Schichtebene und senkrecht zur Schichtebene ablesen kann. Aus dieser Dehnung kann nun mit Hilfe der linearen Elastizitätsttheorie die Spannung in der Schicht bestimmt werden, allerdings ist hierfür die Kenntnis des Biaxialmoduls der Schicht notwendig. Für nicht kubische Strukturen ist es mit dieser Methode ebenfalls möglich, das Achsenverhältnis $c / a$ zu bestimmen, aber hieraus kann nicht direkt die Dehnung und damit die Spannung in der Schicht bestimmt werden. 


\subsection{Elektronenmikroskopie}

Um einen Überblick über die Morphologie der Schichten zu erhalten, bietet das Rasterelektronenmikroskop (REM) ideale Voraussetzungen. Mit dieser Methode können einerseits Bilder mit Kantenlängen von einigen Millimetern aufgenommen werden, andererseits können auch Strukturen von deutlich unter $50 \mathrm{~nm}$ noch aufgelöst werden. Hierbei wird die Probe mit einem sehr fein gebündeltem Elektronenstrahl abgerastert, dessen Wechselwirkung mit der zu untersuchenden Probe verschiedene Informationsquellen eröffnet. Typischerweise werden die rückgestreuten Elektronen oder die aus der Probe ausgelösten Sekundärelektronen detektiert. Zusätzlich zu einem Topographiekontrast der Sekundärelektronen erhält man aus den rückgestreuten Elektronen einen Materialkontrast, da die Zahl der gestreuten Elektronen auch von der Ladungszahl des streuenden Atomkerns abhängt.

Die chemische Zusammensetzung einer Probe ist über energiedispersive Röntgenspektroskopie(EDX) zugänglich: Hierbei wird die Energie der vom Elektronenstrahl in der Probe erzeugten Röntgenstrahlung ausgewertet. Die Linien dieses Spektrum lassen sich verschiedenen elektronischen Übergängen der Atome der Probe zuordnen, so dass aus ihrem Intensitätsverhältnis über eine Datenbank die relative Häufigkeit der einzelnen Elemente bestimmt werden kann. Diese Informationen stammen aus einem Volumen, dass einige Mikrometer in die Tiefe der Probe reicht, so dass bei dünnen Filmen weitere Korrekturfaktoren berücksichtigt werden müssen, und sich bei Schichtdicken von unter einem Mikrometer die Zusammensetzung nur bis auf einige Prozent genau bestimmen lässt.

Je nach dem ob nun ein hochaufösendes Bild oder ein EDX-Spektrum aufgenommen werden soll, unterscheiden sich die Betriebsparameter des REM deutlich: Bei geringen Spannungen ( $5 \mathrm{kV}$ und darunter) und kleinen Arbeitsabständen ( $5 \mathrm{~mm}$ und weniger) ist eine deutlich höhere Auflösung zu erzielen als bei $13 \mathrm{~mm}$ Arbeitsabstand und $20 \mathrm{kV}$ Beschleunigungsspannung, bei denen die EDX-Spektren aufgenommen werden. Um die Zählstatistik und damit die Genauigkeit der Analyse zu verbessern, wird zusätzlich eine größere Blende verwendet, die die Ortsauflösung weiter reduziert.

Während das REM im Wesentlichen die Probenoberfläche abbildet, bietet das Transmissions-Elektronenmikroskop (TEM) einen direkteren Zugang zur Mikrostruktur im Volumen der Probe. Um eine Probe durchstrahlen zu können, müssen aus den Proben dünne Lamellen präpariert werden. Die hier untersuchten Proben wurden mit einem fokussierten Ionenstrahl (FIB) bearbeitet. Dazu wird die Probe mit einem sehr stark fokussierten Strahl aus Gallium-Ionen beschossen (Energie $30 \mathrm{keV}$ ), der lokal Material von der Probe abträgt. Hierfür wird zunächst über der zu untersuchenden Stelle ein Steg aus 

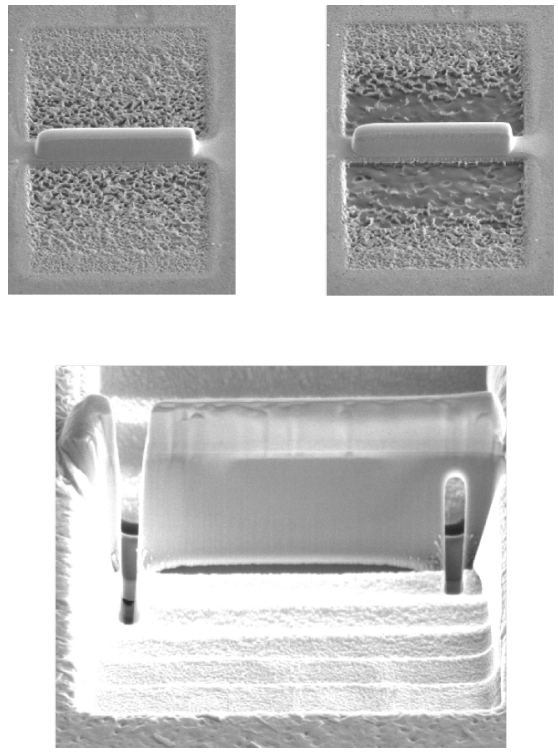
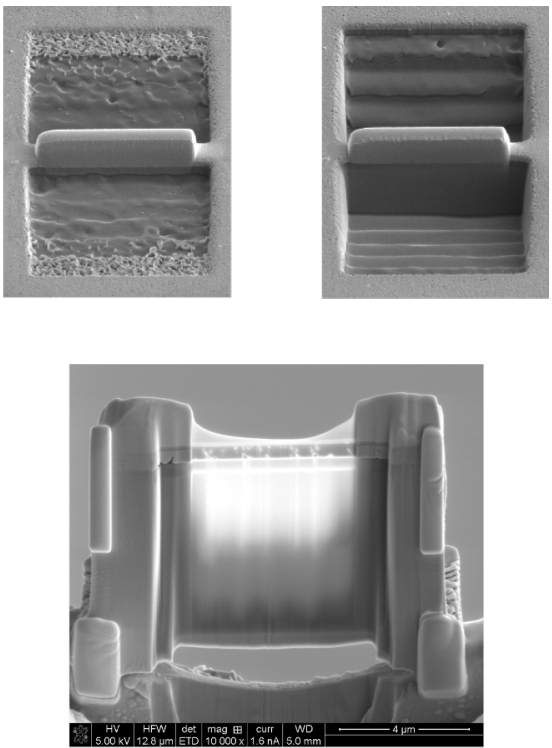

Abbildung 3.12: Verlauf der Lamellenpräparation mit dem FIB: Oben: Neben der Lamelle werden stufenförmig zwei Vertiefungen erzeugt, so dass die Lamelle ausgeschnitten werden kann (unten links). Unten rechts: die fertige Lamelle.

Platin deponiert und dann an beiden Seiten der Lamelle das Material stufenförmig abgetragen (Abbildung 3.12, obere Reihe). Danach kann die Lamelle ausgeschnitten (Abbildung 3.12, unten links), an einem TEM-Probenhalter befestigt und mit dem Ionenstrahl weiter gedünnt werden (Abbildung 3.12, unten rechts).

\subsection{Rastersondenverfahren}

Raster-Elektronenmikroskope liefern hochaufgelöste Abbildungen der Oberflächenmorphologie, dem erzielten Kontrast kann allerdings prinzipbedingt keine Höhenskala zugeordnet werden. Hierfür werden verschiedene Rastersondenverfahren eingesetzt, die alle die Oberfläche der Probe mit einer Spitze abrastern, und dabei die Höhe der Probe bestimmen. Beim Rastertunnelmikroskop (STM) wird dazu zwischen Spitze und Probe eine Spannung von typischerweise unter einem Volt angelegt und die Spitze so lange an die Probe angenähert, bis ein Tunnelstrom im Nanoampèrebereich fließt. Dieser Tunnelstrom hängt exponentiell vom Abstand zwischen Spitze und Probe ab, so dass entweder bei konstanter Höhe der Verlauf des Tunnelstroms gemes- 
sen wird (constant-height-mode), oder - wie sehr viel häufiger- im constantcurrent-mode gemessen wird. Bei diesem wird durch eine Regelschleife die Spitzenhöhe so anpasst, dass immer ein konstanter Tunnelstrom fließt. Beim Rasterkraftmikroskop (AFM) ist die Spitze an einer kleinen und extrem empfindlichen Blattfeder befestigt, deren Verbiegung gemessen wird. Auch hier kann über einen Regelkreis, der eine konstante Verbiegung einregelt, direkt die Topographie der Probe vermessen werden. Darüber hinaus gibt es weiter entwickelte Verfahren, wie den Tappping Mode, bei dem die Spitze zum Schwingen angeregt wird und nur im unteren Umkehrpunkt die Probenoberfläche berührt.

Mit einem sehr ähnlichen Aufbau wie einem AFM, einem so genannten Nanoindenter lassen sich lokal die Härte und elastische Eigenschaften eine Probe untersuchen: Hierfür wird eine pyramidenförmige Spitze in die Probe gedrückt, und aus dem Zusammenhang zwischen Eindringtiefe und Auflagekraft wie der Bestimmung der Vickershärte der Inentationsmodul bestimmt. Die kann entweder quasistatisch (also durch langsames Erhöhen der Auflagekraft) oder dynamisch geschehen. In diesem Fall wird die Auflagekraft zusätzlich mit einem Sinus mit konstanter (kleiner) Amplitude überlagert, so dass über die gesamte Messung hinweg kontinuierlich die Kontaktsteifigkeit (und daraus Härte und elastischer Modul in Abhängigkeit der Tiefe) bestimmt werden können [87].

\subsection{Rutherford-Rückstreuung}

Die Rutherford-Rückstreu-Spektrometrie (RBS) erlaubt es tiefenabhängige Konzentrationsprofile dünner Schichten zu vermessen. Hierfür wird die Probe mit einem Helium-Ionenstrahl mit einer festen Energie im MeV-Bereich beschossen und die Energie der rückgestreuten Ionen gemessen. Betrachtet man nun eine Kollision zwischen einem Heliumkern des Ionenstrahls und einem Atom der Probe, hängt die Energie des rückgestreuten Heliumkerns vom Streuwinkel und wegen Impuls- und Energieerhaltung von den Massen von Projektil $m_{p}$ und Targetatom $m_{t}$ ab. Der kinematische Faktor $k$ (siehe Gleichung 2.9) beschreibt den Zusammenhang zwischen der Energie des einfallenden Heliumkerns $E_{0}$ und der Energie des gestreuten Projektils $E_{1}$. Er vereinfacht sich für einen Streuwinkel von $\Theta=180^{\circ}$ und $m_{t}>m_{p}$ zu:

$$
\frac{E_{1}}{E_{0}}=\frac{m_{t}-m_{p}}{m_{t}+m_{p}}
$$

Aus der Energie eines gestreuten Projektilatoms lässt sich somit die Masse des Atoms bestimmen, an dem es gestreut wurde. Findet solch ein Streupro- 


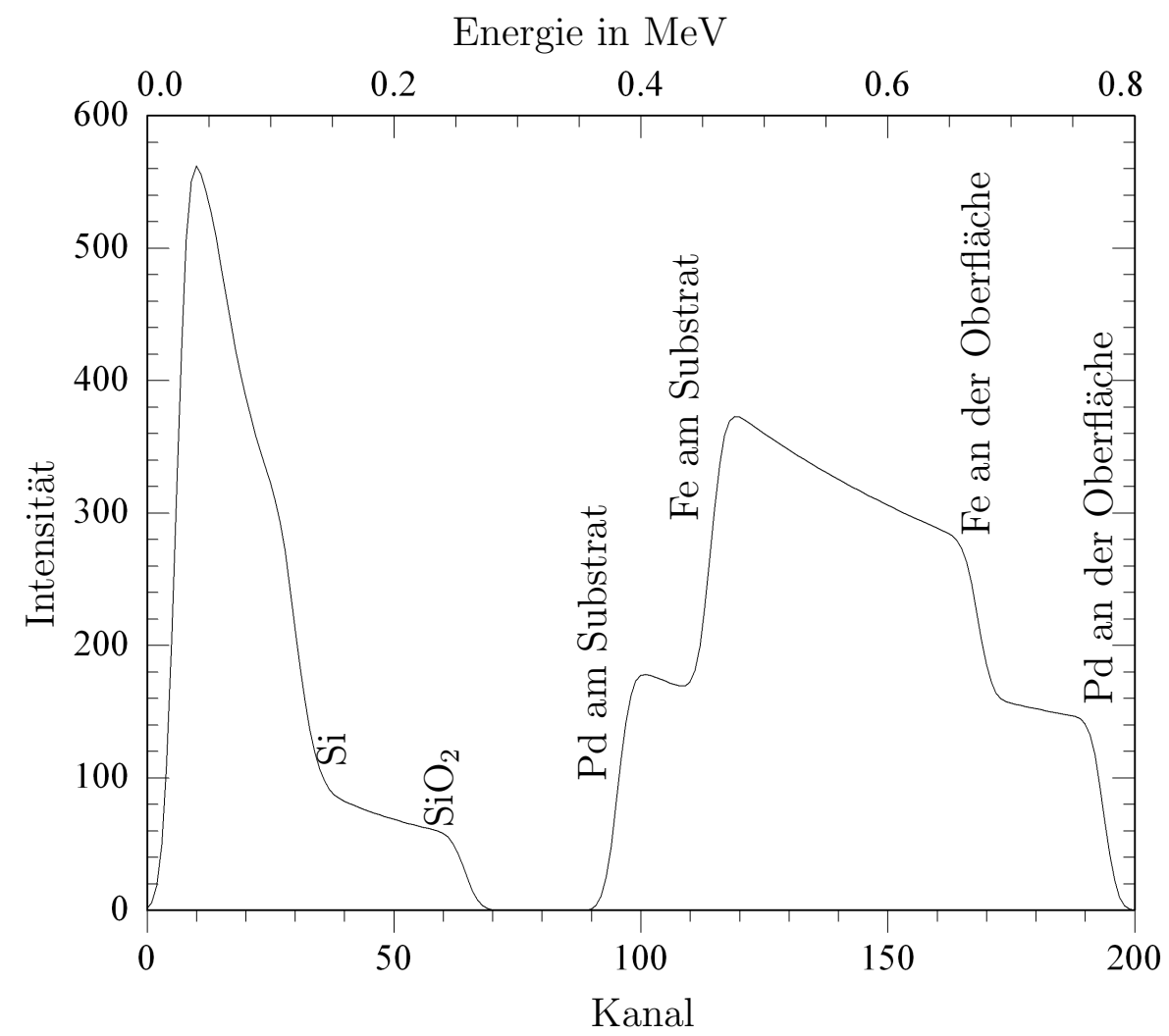

Abbildung 3.13: Mit RUMP [88] simuliertes RBS-Spektrum einer FePdSchicht auf einem Siliziumdoixid-Substrat.

zess nicht an der Probenoberfläche sondern in einer gewissen Tiefe statt, ist zusätzlich der Energieverlust $S(E)$ des Ions beim Durchdringen der Probe zu berücksichtigen, der im Wesentlichen von der Dichte der Probe abhängt.

Diese Spektra lassen sich bei gegebenen Materialparametern mittels der Software RUMP [88] berechnen und mit gemessenen Spektren verglichen, um dann durch eine Variation der Simulationsparameter die Spektren in Übereinstimmung zu bringen. Ein RBS-Spektrum einer dünnen FePd-Schicht auf einem Siliziumsubstrat mit Oxidschicht wie in Abbildung 3.13 gezeigt, besteht also aus zwei Beiträgen: Zum einen aus einem Bereich mit einer Energie zwischen der maximal möglichen Energie für ein Eisen- oder PalladiumTargetatom (Streuung an einem Metallatom an der Oberfläche), und der Energie, die ein Heliumkern hat, der an einem Metallatom direkt an der Grenzfläche zum Substrat gestreut wurde, also zweimal den Energieverlust beim Durchtritt durch die komplette Schichtdicke erlitten hat. Zum anderen 
werden auch Heliumkerne am Substrat gestreut: Die maximale Energie von Atomen, die an Silizium gestreut wurden, ist ebenfalls durch den Energieverlust beim zweimaligen Passieren der Metallschicht sowie dem kinematischen Faktor für Silizium gegeben. Dieser Bereich reicht nun meist bis zur niedrigsten detektieren Energie, da eine Streuung an Siliziumatomen auch in größeren Tiefen möglich ist. Je nach Energie und Massenverhältnis ist es auch möglich, dass diese verschiedenen Beiträge zum Spektrum überlappen. Eine Streuung an den Sauerstoff-Atomen der Siliziumdioxid-Schicht auf dem Substrat ist wegen der geringen Masse von Sauerstoff nicht zu erkennen, allerdings zeigt sich die Grenzfläche zwischen Siliziumdioxid und Silizium als deutliches Ansteigen des Signals, da hier die Dichte der Siliziumatome stark ansteigt.

\subsection{Magnetisierungsmessungen}

Die Messung der magnetischen Eigenschaften der Proben erfolgte mit einem $\mathrm{MPMS}^{10}$. Dieses Messsystem besteht aus einem Helium-gekühlten Dewar, in dem sich ein supraleitender Magnet, der Flussdichten von bis zu 5 Tesla erreichen kann, und ein supraleitendes Quanteninterferenzgerät (SQUID) befinden. Über einen Heizer ist es möglich, die Probe auf beliebige Temperaturen zwischen wenigen Kelvin und $125^{\circ} \mathrm{C}$ zu heizen. Die Magnetisierung der Probe wird nun gemessen, in dem die Probe durch eine Messspule (Secondary Deriviative Detector Array) im Inneren des supraleitenden Magneten bewegt wird. Aus der Messspule wird der induzierte Strom über einen Isolationstransformator ausgekoppelt um dann vom SQUID gemessen zu werden, wie in Abbildung 3.14 dargestellt. Das SQUID selbst besteht aus einem supraleitenden Ring, der durch einen Josephson-Kontakt unterbrochen ist. Dieser Josephson-Kontakt ist eine dünne, isolierende Schicht, durch die die Cooper-Paare des supraleitenden Rings hindurchtunneln können. Hierbei entsteht eine Phasenverschiebung zwischen den Wellenfunktionen, mit denen die Cooper-Paare vor und nach dem Durchtunneln der Barriere beschrieben werden. Diese Phasendifferenz führt nun zur Interferenz der Wellenfunktionen, die somit einen oszillierenden Strom in dem Ring erzeugen. Durch die Messung dieses Stroms kann nun die Flussänderung in der Pickup-Spule und damit das magnetische Moment der Probe bestimmt werden.

\footnotetext{
${ }^{10}$ Magnetic Properties Measurement System,Quantum Design
} 


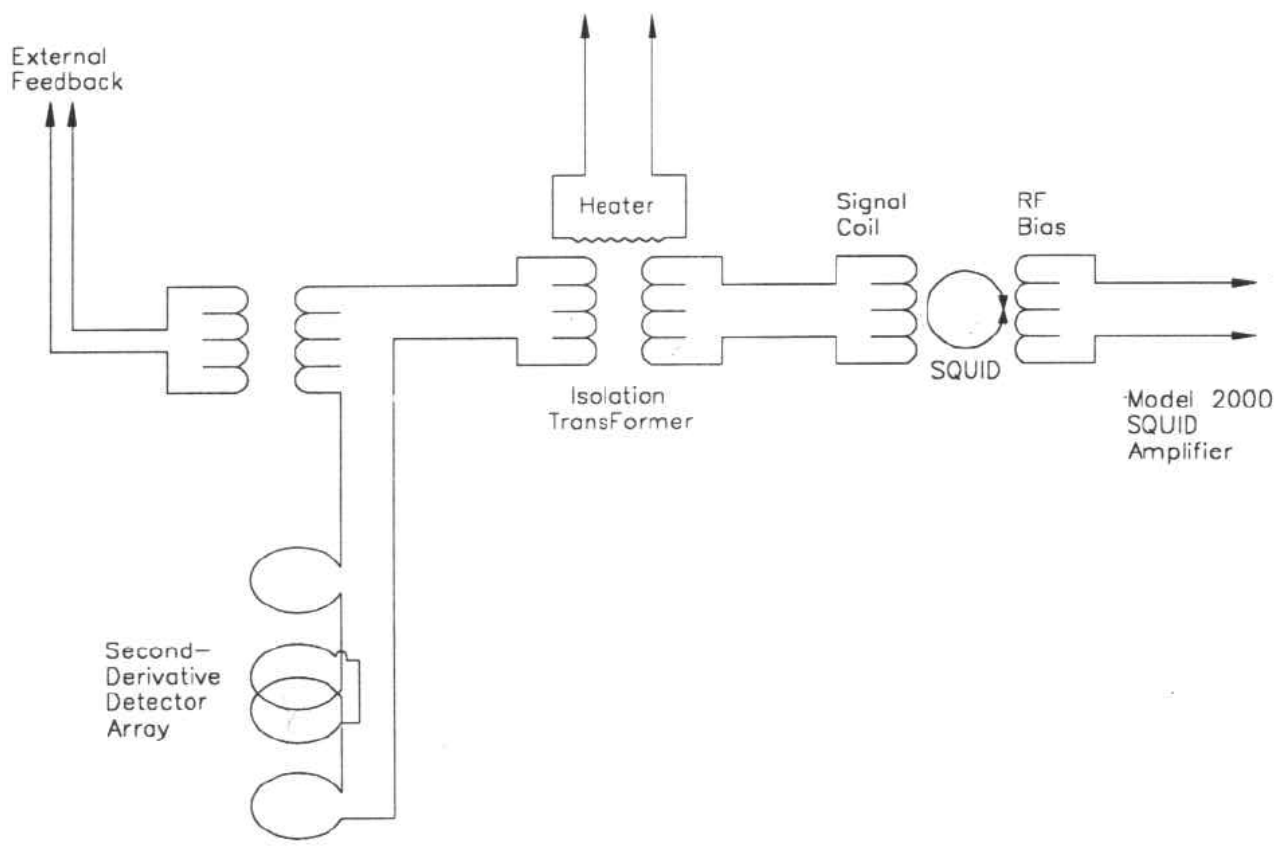

Abbildung 3.14: Schemazeichnung der Magnetisierungsmessung im MPMS: Die Probe wird durch den Detector Array bewegt und der hierbei induzierte Strom über den Isolationstransformator ausgekoppelt. Er erzeugt seinerseits ein Magnetfeld im SQUID, dass dann sehr genau vermessen werden kann. Aus [89] 


\section{Kapitel 4}

\section{Molekulardynamiksimulationen}

Einen völlig anderen Zugang zu mikroskopischen Prozessen bieten klassische Molekulardynamiksimulationen (MD-Simulationen): Hierbei werden die Wechselwirkungen von einzelnen Atomen simuliert, und deren Trajektorien und zahlreiche daraus abgeleitete statistische und mechanische Größen betrachtet. Molekulardynamiksimulationen gehen zunächst immer von einem Potential aus, das die Wechselwirkung zwischen einzelnen Atomen beschreibt:

$$
m_{i} \frac{\mathrm{d}^{2} x_{i}}{\mathrm{~d} t^{2}}=\nabla_{x_{i}} V\left(x_{1}, \ldots, x_{N}\right) \quad i=1,2, \ldots, N
$$

Aus diesem Potential wird die Kraft bestimmt, die auf ein Atom wirkt, und hiermit die Newtonsche Bewegungsgleichug aufgestellt, die dann mit Hilfe eines diskreten Integrationsschemas gelöst werden kann.

\subsection{Verwendetes Potential}

Im Rahmen dieser Arbeit wurde das von S. G. Mayr auf Basis früherer Versionen von parcas und alcmd weiterentwickelte Programm mdsim benutzt, das unter anderem auch von Neudecker in [90] verwendet wurde, und einen Gear Predictor-Corrector Intergationsagorithmus 5. Ordnung zur Lösung der Bewegungsgleichungen verwendet [91, 92, 93].

Die ersten für Molekulardynamiksimulationen verwendeten Potentiale waren reine Paarpotentiale (wie z. B. das Lennard-Jones-Potential), die den Vorteil haben, dass nur über alle Atompaare iteriert werden muss. Allerdings beschreibt ein solches Potential nur vollständig ungerichtete Bindungen zwischen den Atomen und kann daher nicht alle Kristallstrukturen wiedergeben. Komplexere Potentiale wie das Stillinger-Weber-Potential [94] beinhalten auch Terme, die von dem Winkel zwischen zwei Bindungen abhängen, 
und erfordern daher in jedem Zeitschritt eine Iteration über alle Atomtripel. Dies erhöht zwar den numerischen Aufwand immens, ist aber notwendig um den Charakter von kovalenten Bindungen zu beschreiben. Durch geeignete Verfahren ist es möglich, diesen Mehraufwand zu verringern: So können zum Beispiel Listen angelegt werden, welche Atome sich in der Nachbarschaft eines bestimmten Atoms befinden. Soll nun die Kraft auf dieses Atom bestimmt werden, genügt es statt aller Atome der Simulationszelle nur dessen Nachbarn zu betrachten. Da die Atome sich im Lauf der Simulation bewegen können, müssen auch die Nachbarschaftslisten immer aktuell gehalten werden, trotzdem kann so der Bedarf an Rechenzeit dramatisch reduziert werden.

Das in diesen Simulationen verwendete Potential wurde von Müller, Erhart und Albe [95] entwickelt und folgt dem Angular Dependet Analytic Bond-order Potential (ABOP) Formalismus [96, 97]. Es enthält sowohl EAMTerme, bei denen ein einzelnes Atom in einem effektiven Potential, das aus den gemittelten Elektronendichten aller anderen Atome besteht, betrachtet wird [98], als auch Terme, die vom Winkel zwischen den Bindungen abhängen.

Für die im Rahmen dieser Arbeit durchgeführten Simulationen wurde das System Eisen-Platin als Modellsystem gewählt. Für dieses Materialsystem existiert ein etabliertes Wechselwirkungspotential [95], und es ist zu erwarten, dass wegen der großen chemischen und strukturellen Ähnlichkeit der Systeme Eisen-Platin und Eisen-Palladium die Ergebnisse qualitativ übertragbar sind. Quantitativ lassen sich die Simulationsergebnisse nicht direkt auf experimentelle Werte übertragen, da dieses Potential dafür bekannt ist, zu hohe Werte für die Verzwillingungsenergie zu liefern [95].

\subsection{Ablauf der Simulationen}

Als Referenzsystem wurde eine quaderförmige Simulationszelle aus 250.000 Eisen- und 250.000 Platinatomen in der $\mathrm{L}_{0}$-Struktur hergestellt und mit einem Berendsen-Thermostat und Barostat [99] auf eine Temperatur von 900 K aufgeheizt. Nachdem die Zelle ohne äußeren Druck für 200 ps relaxiert wurde, wurden die periodischen Randbedingungen in $z$-Richtung aufgehoben und die Atome an der unteren Grenzfläche festgehalten, so dass diese nun ein unendlich steifes Substrat bilden. Die obere Grenzfläche stellt dagegen eine freie Oberfläche dar. Der so erzeugte Film wird nun bei konstantem Volumen weitere 40 ps relaxiert und erreicht während dieser Zeit eine konstante Gesamtenergie. Um den Einfluss von Defekten, insbesondere Eisenausscheidungen zu untersuchen, wurde analog eine weitere Zelle hergestellt, bei der einige Bereiche des FePt-Kristalls entfernt und durch bcc-Eisen ersetzt wur- 
den.

\subsection{Der Zentralsymmetrie-Parameter}

Zur Visualisierung der strukturellen Änderungen während der Simulation wurde hauptsächlich der Zentralsymmetrie-Parameter $c$ verwendet, der mit der Visualisierungssoftware AtomEye [100] berechnet wurde. Dieser Wert wird in [101] für fcc-Gitter definiert, und wird von der Software nach einem verallgemeinerten Verfahren für beliebige Strukturen bestimmt:

Zunächst kann man die beiden trivialen Fälle von einem einzelnen Atom und einem einzelnen Atompaar behandeln: Falls ein Atom $i$ keine Nachbarn hat, wird $c_{i}$ der Wert 0 zugewiesen, da ein einzelnes Atom immer eine perfekt punktsymmetrische Anordnung darstellt. Hat ein Atom genau einen Nachbarn, ist die Punktsymmetrie maximal gebrochen und man definiert $c_{i}=1$.

Für alle anderen Atome berechnet sich $c_{i}$ folgendermaßen:

Zunächst wird die maximale Zahl der Nachbaratome $M$ aus der häufigsten Zahl der Nachbaratome $N_{h}$ bestimmt. Da das Verfahren immer Atompaare betrachtet, wird diese Zahl durch Zwei geteilt, die Nachkommastellen abgeschnitten, und wieder mit Zwei multipliziert:

$$
M=\left\lfloor\frac{N_{h}}{2}\right\rfloor \cdot 2
$$

Für ein fcc-Gitter erhält man so $M=12$, für ein bcc-Gitter $M=8$. Mit der Zahl der Nachbarn $N_{i}$ des $i$-ten Atoms kann man nun definieren:

$$
\tilde{m}_{i}=\min \left(M, N_{i}\right)
$$

Die Fälle $\tilde{m}_{i}=0$ und $\tilde{m}_{i}=1$ wurden bereits ausgeschlossen, und für alle $\tilde{m}_{i} \geq 2$ definiert man, um wieder nur gerade Zahlen zu erhalten:

$$
m_{i}=\left\lfloor\frac{\tilde{m}_{i}}{2}\right\rfloor \cdot 2
$$

Nun sortiert man die $j=1 . . N_{i}$ Nachbarn eines Atoms nach deren Abständen $\left|\vec{d}_{j}\right|$ von Atom $i$ in aufsteigender Reihenfolge, betrachtet hiervon nur die ersten $m_{i}$ Atome.

Man nimmt nun den Nachbarn, der den kleinsten Abstand (also $\overrightarrow{d_{1}}$ ) von Atom $i$ hat und sucht aus den verbliebenen Nachbarn dasjenige Atom $j$ für das

$$
D_{1}=\left|\overrightarrow{d_{1}}+\vec{d}_{j}\right|^{2}
$$




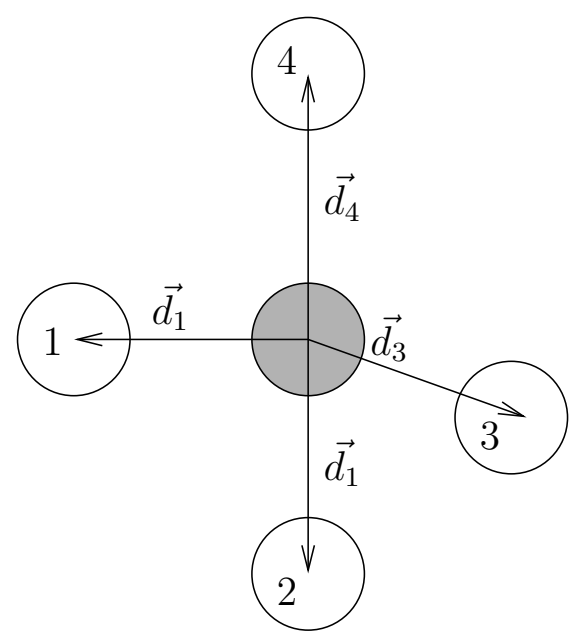

Abbildung 4.1: Beispiel zur Berechnung des Zentralsymmetrie-Parameters.

minimal wird, das also am ehesten dem Atom mit Abstand $\vec{d}_{1}$ gegenüber liegt.

Nun entfernt man die Atome 1 und $j$ aus der Menge, und bestimmt $D_{2} \ldots D_{m_{i} / 2}$ analog.

Der Zentralsymmetrie-Parameter beträgt dann:

$$
c_{i}=\frac{\sum_{k=1}^{m_{i} / 2} D_{k}}{2 \sum_{j=1}^{m_{i} / 2}\left|\vec{d}_{j}\right|^{2}}
$$

Was dieser Parameter anschaulich bedeutet, soll hier an einem zweidimensionalen Beispiel in Abbildung 4.1 verdeutlicht werden:

Das grau markierte Atom hat 4 Nachbarn, also beträgt $m=4$. Nun beginnt man mit dem Nachbarn, der den kleinsten Abstand hat, (also in diesem Fall Atom 1) und sucht dasjenige Atom $j$, für das die Summe der beiden Verbindungsvektoren vom Ausgangsatom $D_{1}=\left|\overrightarrow{d_{1}}+\overrightarrow{d_{j}}\right|^{2}$ minimal wird. Dies ist für Atom 3 der Fall. Wären nun Atom 1 und 3 genau symmetrisch bezüglich des Ausgangsatoms, wäre $D_{1}=0$. Da sie es in diesem Beispiel nicht sind, ergibt sich ein endlicher Wert. Nun sind die Atome 1 und 3 behandelt und werden aus der Liste der Nachbarn gestrichen. Von den verbleibenden Atomen wird nun wieder das gewählt, dass den geringsten Abstand zum Ausgangsatom hat und auch für dieses ein anderes Atom gesucht, so dass $D_{2}=\left|\vec{d}_{2}+\vec{d}_{j}\right|^{2}$ minimal wird (in diesem Fall also Atom 4). Auch 
diese beiden Atome werden aus der Liste der Nachbarn entfernt. Da nun alle Nachbarn behandelt sind, werden alle $D_{j}$ aufsummiert und durch die Summe der Bindungslängen geteilt.

In einem perfekten zentralsymmetrischen Kristall ist immer $c_{i}=0$, da hier von einen Zentralatom aus immer zwei Atome exakt gegenüber liegen, und damit alle $D_{k}$ Null sind. Für einen Stapelfehler ABC|BCA in einem fccKristall ist in den beiden Atomlagen $\mathrm{C} \mid \mathrm{B}$ die Symmetrie gebrochen, so dass $c_{i}$ hier einen festen Wert hat:

$$
c_{i}=\frac{3 \cdot 0+3 \cdot(d \sqrt{3} / 2 \cdot 1 / 3 \cdot 2)^{2}}{2 \cdot 12 d^{2}}=\frac{1}{24} \approx 0,0417
$$




\section{Kapitel 5}

\section{Ergebnisse}

In diesem Kapitel sollen zunächst die experimentellen Ergebnisse zum Wachstum und der Struktur von FePd-Schichten bei verschiedenen Herstellungsmethoden dargestellt werden. In Verbindung mit Untersuchungen der Morphologie und der Mikrostruktur der Schichten liefern hier insbesondere Messungen der Substratkrümmung einen detaillierten Einblick in die mikroskopischen Prozesse beim Wachstum, die danach auch in den Molekulardynamiksimulationen untersucht werden. Im Anschluss werden vom Substrat abgelöste Filme hinsichtlich ihrer elastischen Eigenschaften, ihrer Struktur und deren Änderung durch eine nachträgliche Temperaturbehandlung untersucht. Auf der Grundlage dieser Ergebnisse werden dann für alle drei Arten von Proben (polykristalline Filme, epitaktische Filme und abgelöste Filme) temperaturabhängige Änderungen der Gitterstruktur und deren Auswirkungen auf die elastischen Eigenschaften, die Magnetisierung und den elektrischen Widerstand bestimmt.

\subsection{Polykristalline Schichten}

Beim Wachstum von Eisen-Palladium-Filmen auf Siliziumdioxid bei Raumtemperatur stellt sich die bct-Phase mit einer sehr feinen Mikrostruktur ein $[33,102]$. Um die gewünschte fcc-Struktur zu erhalten, wurde im Rahmen dieser Arbeit das Wachstum auf geheizten Siliziumsubstraten, das Wachstum während einer Ionenbestrahlung und der Einfluss von nachträglichen Temperaturbehandlungen und Ionenbestrahlungen untersucht. 


\subsubsection{Wachstum auf Siliziumdioxid}

Um in den aufgedampften Schichten die fcc-Struktur zu erhalten, ist entweder eine Temperaturbehandlung der Schicht nach der Herstellung notwendig, oder das Substrat wird schon während der Herstellung auf eine Temperatur gebracht, bei der die gewünschte Phase entsteht. Aus dem Phasendiagramm für Bulk Eisen-Palladium [30] in Abbildung 2.1 ist zu erkennen, dass bei $30 \%$ Palladium für Temperaturen oberhalb von $760{ }^{\circ} \mathrm{C}$ die $\gamma$-Phase stabil ist. Das Wachstum dünner Schichten aus der Dampfphase stellt jedoch einen Prozess dar, der weit vom thermischen Gleichgewicht entfernt ist, so dass sich das Gleichgewichts-Phasendiagramm für Bulkmaterialien nicht unbedingt übertragen lässt.

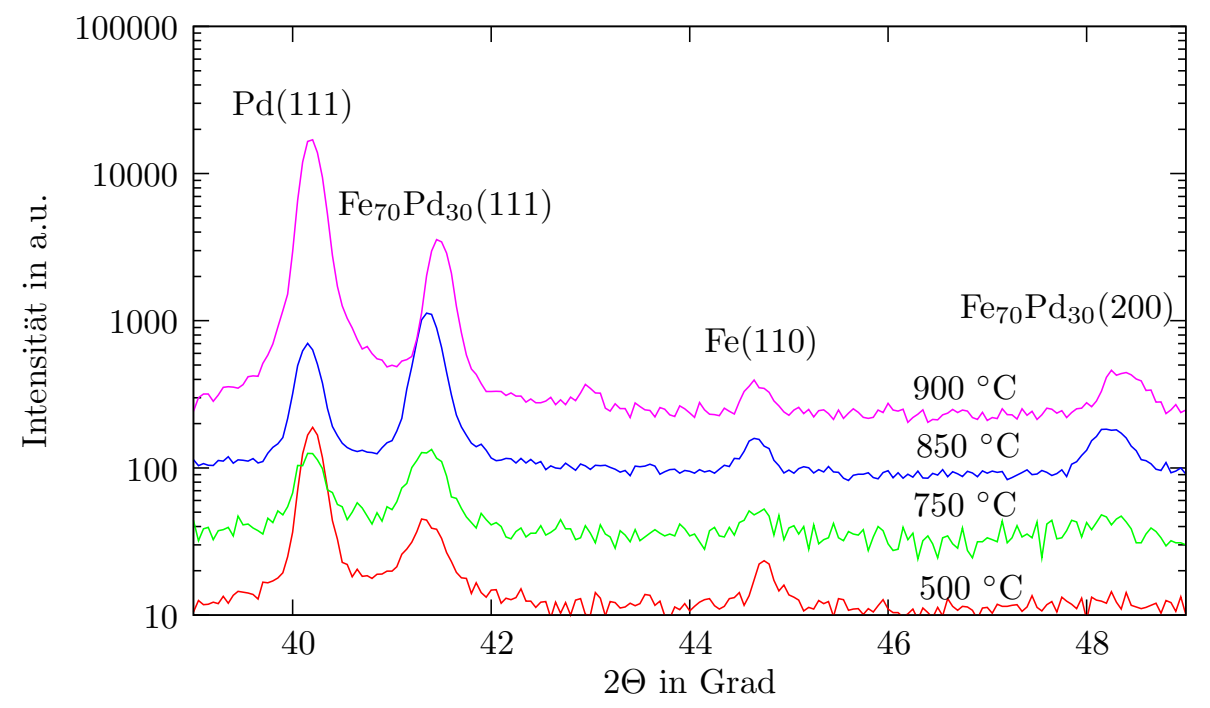

Abbildung 5.1: Röntgendiffraktogramm für $\mathrm{Fe}_{70} \mathrm{Pd}_{30}$-Schichten, die auf $\mathrm{SiO}_{2}$ bei verschiedenen Substrattemperaturen aufgedampft wurden. Alle Proben zeigen neben den $\mathrm{Fe}_{70} \mathrm{Pd}_{30}$-Peaks auch Reflexe von reinem Eisen und Palladium, die Filme sind also teilweise entmischt.

Die Röntgendiffraktogramme für bei verschiedenen Temperaturen aufgedampfte $\mathrm{Fe}_{70} \mathrm{Pd}_{30}$-Schichten in Abbildung 5.1 zeigen neben Reflexen, die sich einer Eisen-Palladium-Legierung zuordnen lassen, auch die charakteristischen Reflexe für reines Palladium und reines $\alpha$-Eisen. Zusätzliche Untersuchungen im Rasterelektronenmikroskop in den Abbildungen 5.2, 5.3 und 5.4 zeigen zudem, dass auch bei einer nominellen Schichtdicke von $100 \mathrm{~nm}$ die Filme noch nicht geschlossen sind, sondern aus einzelnen Inseln bestehen, deren Größe stark von der Substrattemperatur abhängt. Die einzelnen Körner 


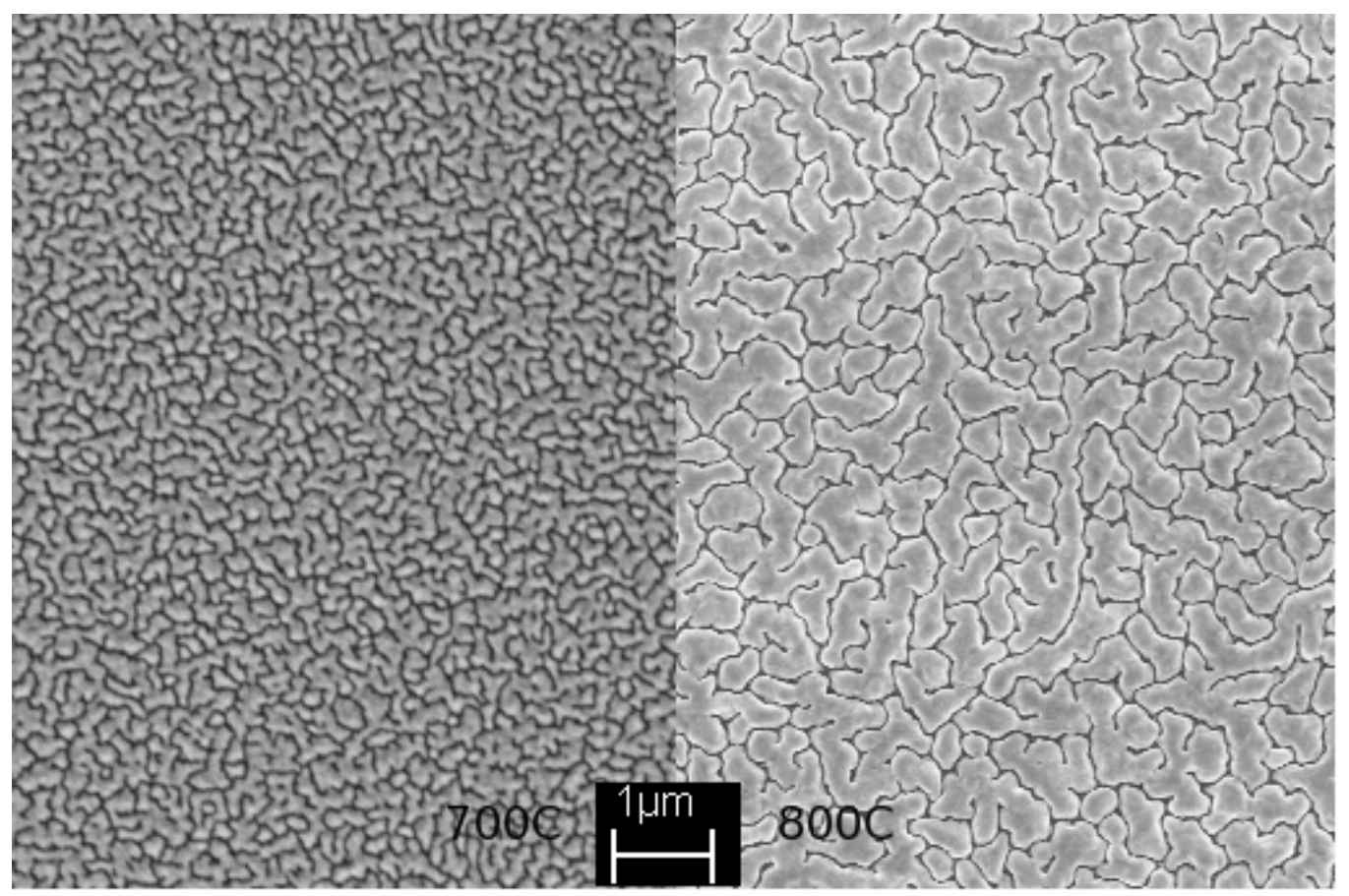

Abbildung 5.2: REM-Aufnahmen von $\mathrm{Fe}_{70} \mathrm{Pd}_{30}$-Schichten, die auf $\mathrm{SiO}_{2}$ bei Substrattemperaturen von $700^{\circ} \mathrm{C}$ und $800^{\circ} \mathrm{C}$ aufgedampft wurden. Trotz einer nominellen Schichtdicke von $100 \mathrm{~nm}$ sind diese Schichten nicht geschlossen.

weisen deutliche Kristallfacetten auf und sind mäanderförmig von Gräben getrennt, in denen mittels eines EDX-Linescans (siehe Abbildung 5.4) ein deutlicher Rückgang der Signale von Eisen und Palladium, und ein gleichzeitiger Anstieg des Siliziumsignals auf über $95 \%$ nachgewiesen werden kann. Auch wenn in diesen Gräben eine Bedeckung des Substrats mit wenigen Monolagen Eisen und oder Palladium anhand dieser Messung nicht ausgeschlossen werden kann, ist davon auszugehen, dass die Gräben bis auf das Substrat reichen. 


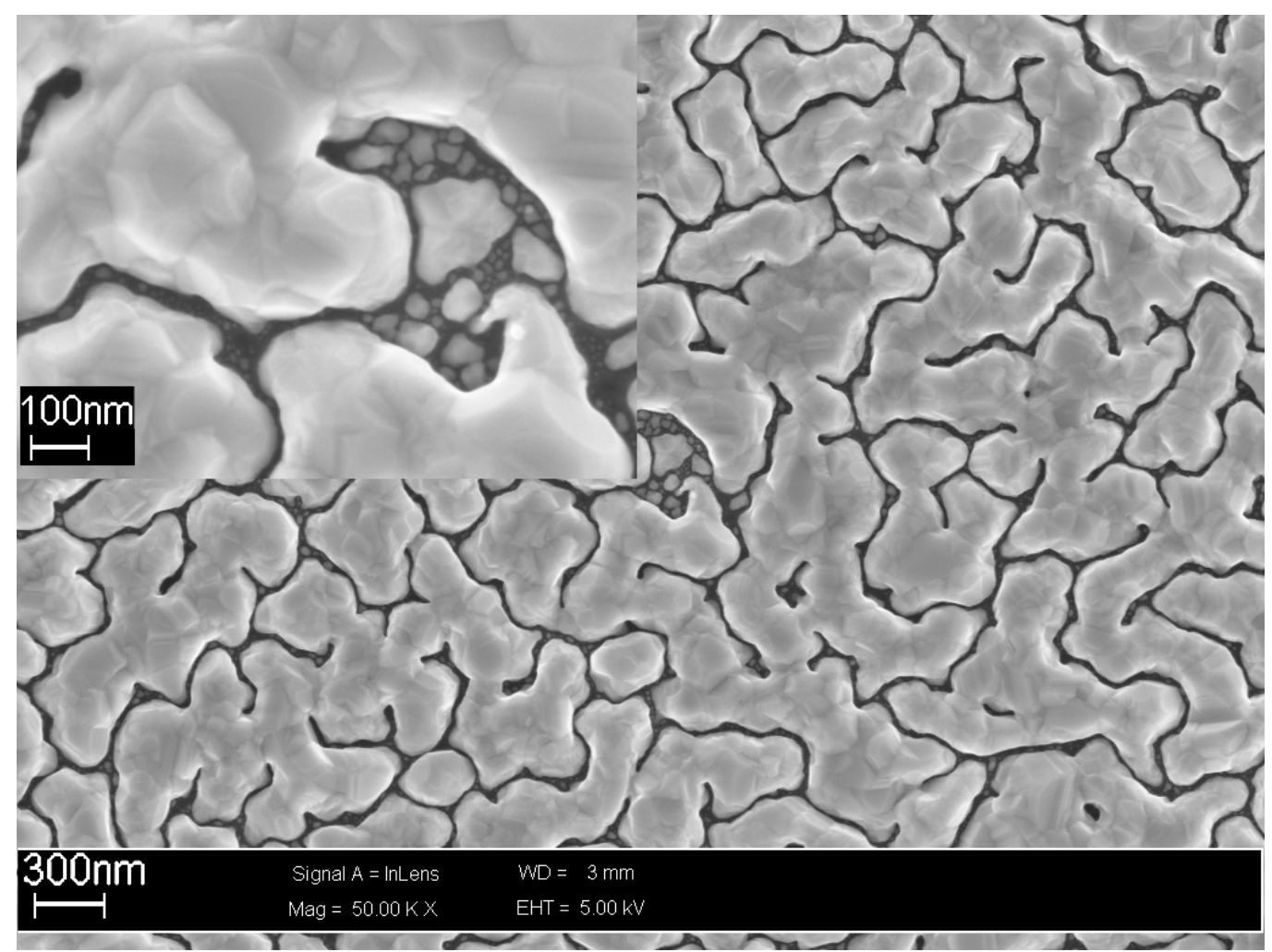

Abbildung 5.3: REM-Aufnahmen einer $\mathrm{Fe}_{70} \mathrm{Pd}_{30}$-Schicht, die auf $\mathrm{SiO}_{2}$ bei einer Substrattemperatur von $850^{\circ} \mathrm{C}$ aufgedampft wurde. Im vergrößerten Inset ist deutlich zu erkennen, dass sich in den Gräben zwischen den großen Inseln weitere, kleinere Inseln befinden, die ihrerseits wieder durch Gräben voneinander getrennt sind. 


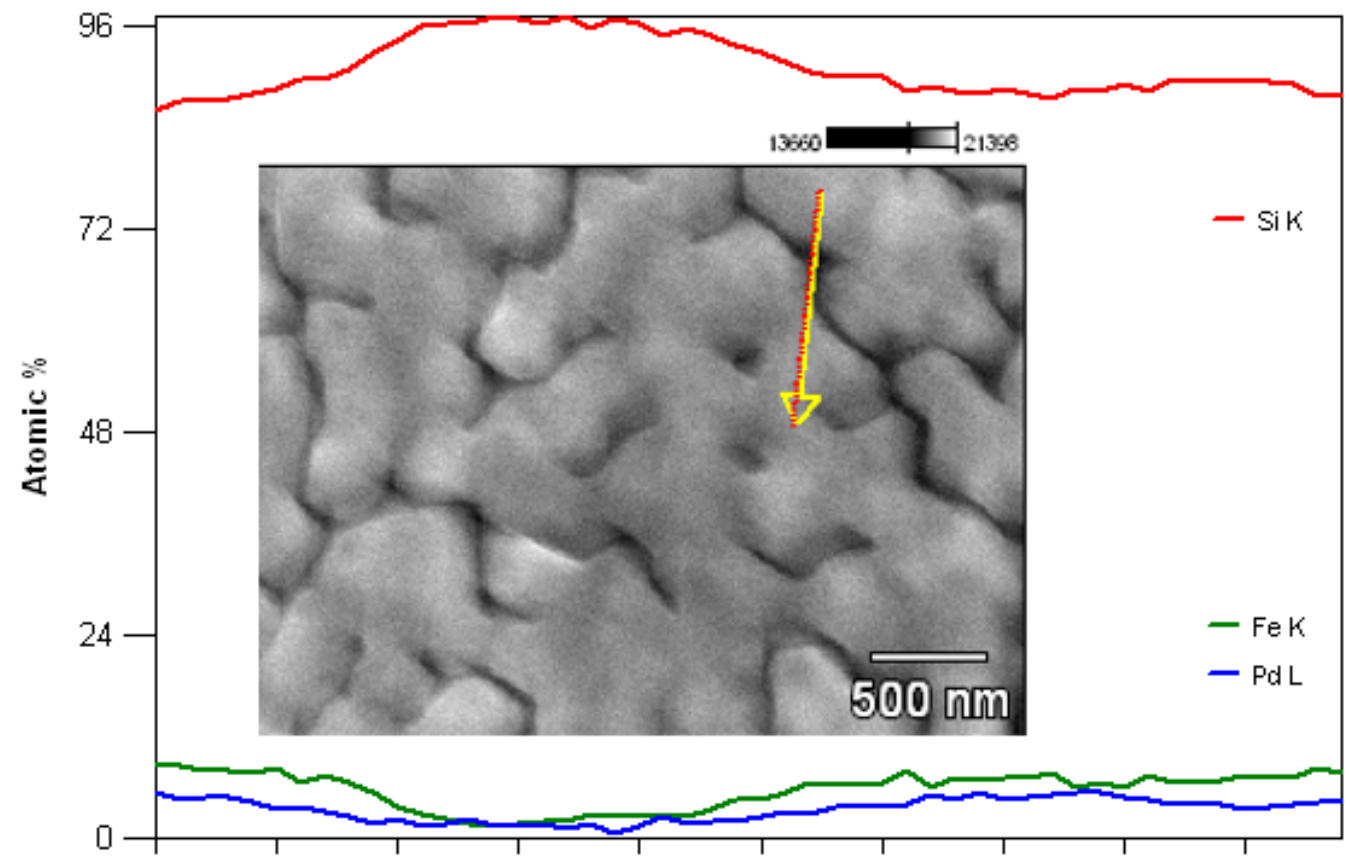

Abbildung 5.4: EDX-Linescan über einen Graben zwischen zwei Inseln einer bei $850{ }^{\circ} \mathrm{C}$ auf $\mathrm{SiO}_{2}$ aufgedampften $\mathrm{Fe}_{70} \mathrm{Pd}_{30}$-Schicht. Inset: Topographie des Bereichs, in dem der Linescan durchgeführt wurde. Im Vergleich zu anderen REM-Aufnahmen ist die Aufösung wegen der für EDX notwendigen Beschleunigungsspannung deutlich geringer. 


\subsubsection{Aufdampfen mit Ionenstrahl-Unterstützung}

Zur Untersuchung des Wachstumsverhaltens unter niederenergetischer Ionenbestrahlung wurden Schichten bei verschiedenen Ionenenergien und $\mathrm{Zu}-$ sammensetzungen auf thermisch oxidierte Siliziumsubstrate aufgedampft. Je nach verwendeten Betriebsparametern der Ionenkanone variiert die tatsächliche Depositionsrate deutlich, so dass nur anhand von Referenzschichten (wie in Kapitel 3.1.3 dargestellt) eine genaue Bestimmung der Schichtzusammensetzung möglich ist. Die Dicke dieser Schichten liegt jeweils zwischen 20 und $40 \mathrm{~nm}$.

Zur Bestimmung der Kristallstruktur wurden an allen Proben Röntgenbeugungsmessungen durchgeführt. Die Proben lassen sich danach in drei Klassen einteilen, nämlich in solche, bei denen nur der bcc(110)-Reflex zu sehen ist, solche, bei denen nur der fcc(111)-Reflex auftritt, und Proben, bei denen beide Reflexe auftreten. Für alle drei Fälle ist jeweils eine repräsentative Messung in Abbildung 5.5 gezeigt.

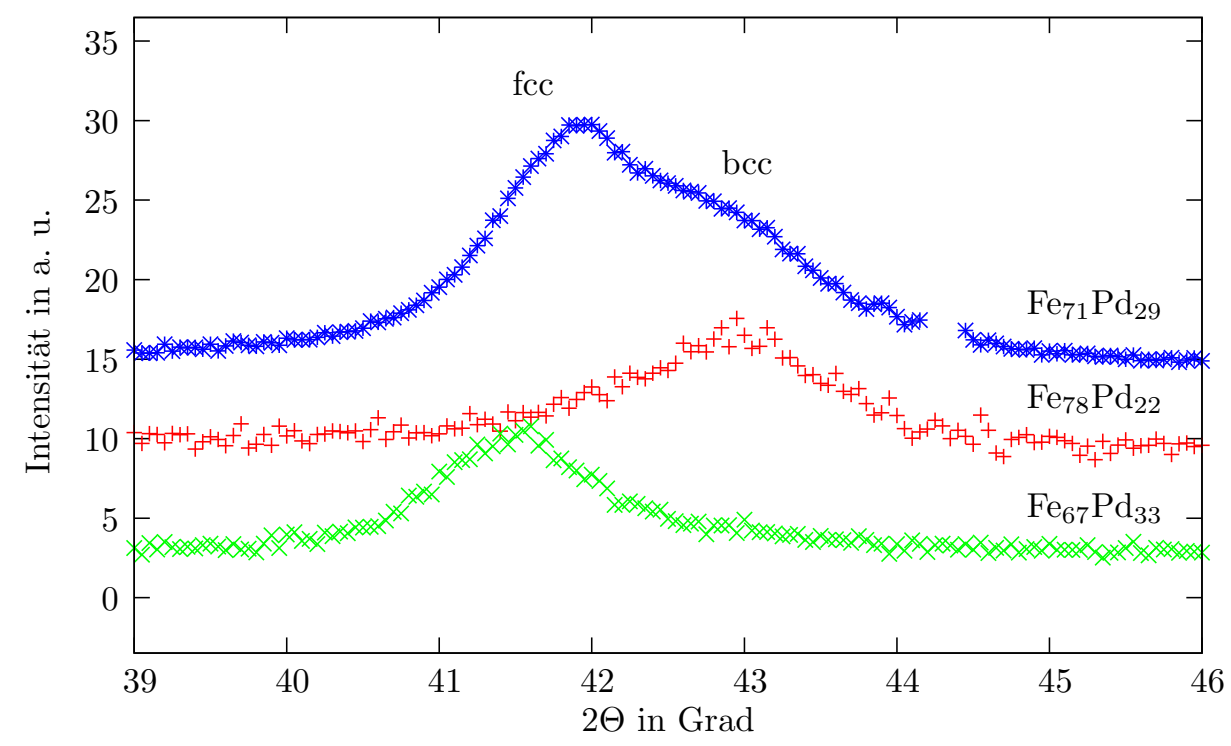

Abbildung 5.5: Beispiele für mit IBAD hergestellte Proben, die verschiedene Gitterstrukturen aufweisen. Bei der Probe, die beide Peaks zeigt, wurde ein Substratpeak bei $44.3^{\circ}$ entfernt.

In dem Parameterbereich, der mit der verwendeten Ionenquelle zugängliche war, zeigen die Untersuchungen keinen systematischen Zusammenhang von Struktur und Ionendosis, und auch Änderungen der Ionenenergie zwischen 30 und 110 eV zeigen nur einen geringen Einfluss. Deutlich zu erkennen 


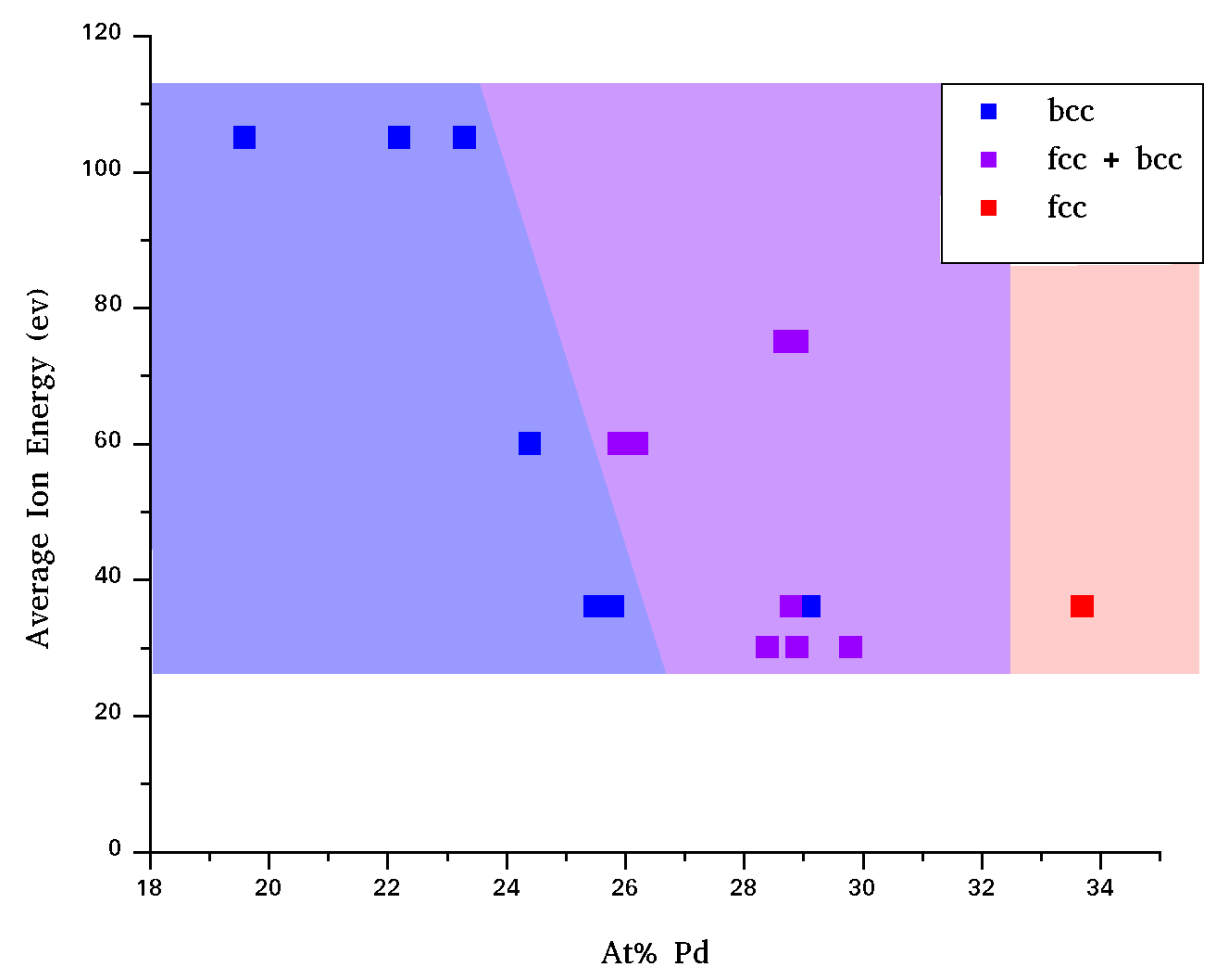

Abbildung 5.6: Phasendiagramm der Filme, die während des Wachstums mit Ionen bestrahlt wurden.

ist in Abbildung 5.6 allerdings der Einfluss der Schichtzusammensetzung: Für sehr geringe Palladium-Konzentrationen (unterhalb $24 \%$ ) beobachtet man die bcc-Phase, für hohe Palladium-Konzentrationen (oberhalb $33 \%$ ) erhält man die fcc-Phase. Dazwischen erstreckt sich ein breiter Bereich, in dem die Diffraktogramme einen stark verbreiterten Peak aufweisen, der als Überlagerung von fcc(111) und bcc(110)-Reflex verstanden werden kann. Zusätzlich wurde auch der Einfluss der Substrattemperatur untersucht. Hierbei stellt man fest, dass für die untersuchten Temperaturen von $200^{\circ} \mathrm{C}$ bis $500{ }^{\circ} \mathrm{C}$ im Gegensatz zu den bei Raumtemperatur hergestellten Schichten immer der (110)-Peak von $\alpha$-Eisen zu beobachten ist, die Schichten also entmischen, wie sie es auch ohne die Ionenbestrahlung tun.

\subsubsection{Nachträgliches Tempern der Filme}

Durch eine Temperaturbehandlung der Schichten nach der Herstellung ist es möglich, die Struktur auch nachträglich noch zu beeinflussen. Im Gegen- 


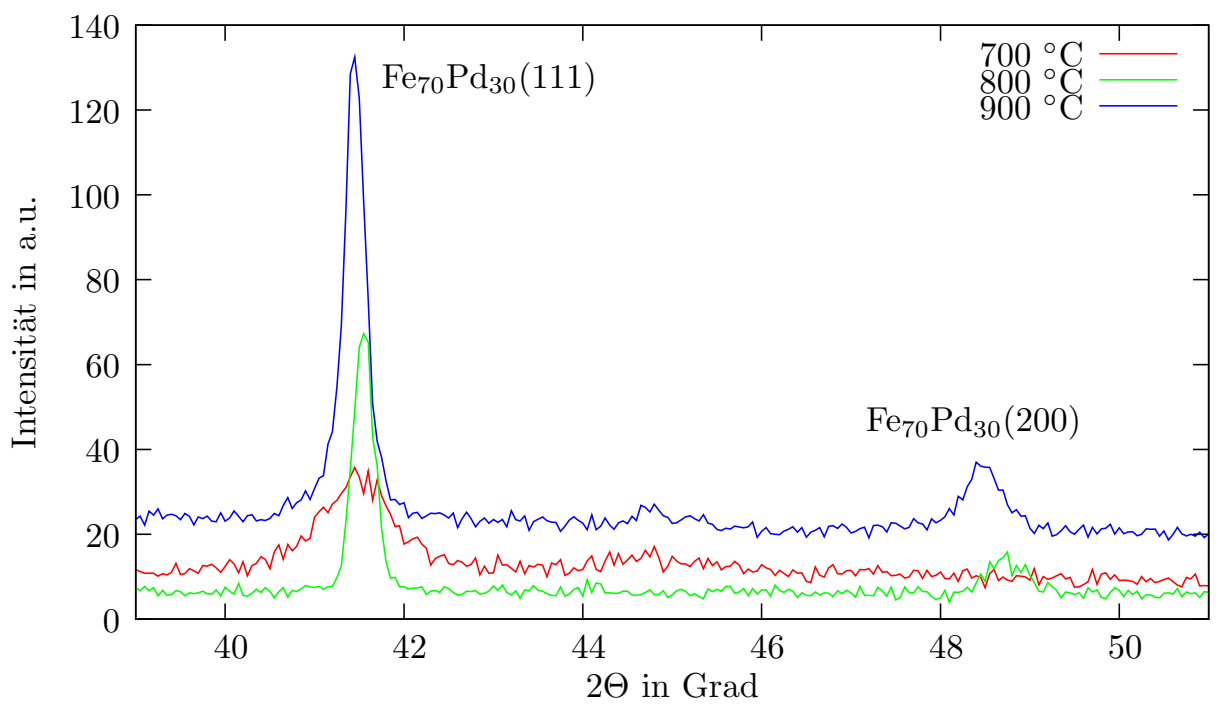

Abbildung 5.7: Durch Tempern von $\mathrm{Fe}_{70} \mathrm{Pd}_{30}$-Schichten bei verschiedenen Temperaturen für jeweils 15 Minuten kann die fcc-Struktur stabilisiert werden. Mit steigender Temperatur werden die Reflexe deutlich schärfer.

satz zum Abkühlen in den jeweiligen Depositionsanlagen besteht hierbei die Möglichkeit die Abkühlrate deutlich höher zu wählen. Wird eine in ein Quarzrohr eingeschmolzene Probe von $1000{ }^{\circ} \mathrm{C}$ in Wasser abgeschreckt, hört diese nach zwei bis drei Sekunden auf zu glühen. Hieraus lässt sich eine Abkühlgeschwindigkeit von 200 bis 300 Grad pro Sekunde abschätzen. Dies ermöglicht es, eine Struktur, die sich bei hoher Temperatur eingestellt hat beizubehalten, ohne dass die Proben entmischen. Für Proben mit einen Palladiumgehalt von $30 \%$ lässt sich so die fcc-Struktur einstellen, bei geringerem Palladiumgehalt kann bei Raumtemperatur auch schon die fct-Struktur vorliegen, wie in Kapitel 5.5.1 dargestellt wird. Sowohl anhand der deutlich schärferen Röntgenreflexe in Abbildung 5.7 als auch anhand von REM-Untersuchungen lässt sich ein massives Kornwachstum von $10 \mathrm{~nm}$ vor der Temperaturbehandlung zu Korngrößen im $\mu \mathrm{m}$ Bereich nach 15 Minuten bei $900^{\circ}$ beobachten.

\subsubsection{Mit hochenergetischen Ionen bestrahlte Filme}

Im Gegensatz zu einer Bestrahlung der Filme während des Wachstums, die nur geringe Ionenenergien erfordert, sind sehr hohe Energien notwendig um die Kristallstruktur zu verändern, wenn die Proben nach der Herstellung bestrahlt werden. Im Rahmen dieser Arbeit wurden von See Wee Chee in der Arbeitsgruppe von R. S. Averback (Department of Materials Science and En- 
gineering, University of Illinois at Urbana-Champaign) $\mathrm{Fe}_{70} \mathrm{Pd}_{30}$-Schichten mit $0,8 \mathrm{MeV} \mathrm{Xe}{ }^{+}$-Ionen bestrahlt. Hierbei wurde eine Probe mit $10^{16}$ Ionen pro $\mathrm{cm}^{2}$ unter senkrechtem Einfall bei Raumtemperatur, eine zweite Probe bei Raumtemperatur mit $10^{16}$ Ionen pro $\mathrm{cm}^{2}$ unter einem Einfallswinkel von $45^{\circ}$, und die dritte Probe mit $10^{16}$ Ionen pro $\mathrm{cm}^{2}$ unter senkrechtem Einfall bei einer Temperatur von $400^{\circ} \mathrm{C}$ bestrahlt. Die in Abbildung 2.14 gezeigten Simulationen mit trim [60] bzw. dessen Nachfolger SRIM [59] zeigen, dass Xenon-Ionen mit einer Energie von $0.8 \mathrm{MeV}$ etwa $200 \mathrm{~nm}$ in eine Eisen-Palladium-Schicht eindringen.

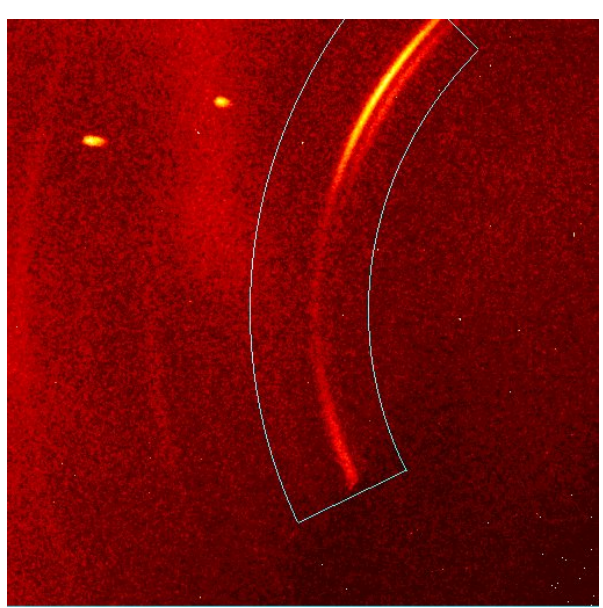

Abbildung 5.8: Intensitätsverteilung auf dem Flächendetektor der bei Raumtemperatur senkrecht bestrahlten Schicht. Die breiten, bogenförmigen Reflexe stammen von der Schicht, die Punktförmigen Reflexe sind dem Substrat zuzuordnen.
Um eine eventuell durch die Bestrahlung entstandene Textur zu untersuchen, wurden an diesen Proben Röntgenbeugungsbilder (siehe Abbildung 5.8) mit einem Flächendetektor aufgenommen. Die beiden bei Raumtemperatur unter verschiedenen Winkeln bestrahlten Proben unterscheiden sich nicht und weisen keine ausgeprägte Textur auf, so dass der Einfallswinkel offensichtlich keinen starken Einfluss auf die Orientierung der Kristallite zu haben scheint. Channeling, also eine erhöhte Ionenreichweite entlang bestimmter Gitterrichtungen [28] ist für Xenon wegen dessen Größe ebenfalls nicht zu erwarten, da Xenon mit 190 pm den größten Pauli-Ionenradius aller Elemente besitzt. Darüber hinaus zeigten diese beiden Proben ebenfalls, dass die Bestrahlungseffekte nicht

stark von der Fluenz (Dosis pro Fläche) abhängen, da hier durch das Schrägstellen der Probe eine größere Fläche bestrahlt wurde.

Die Bestrahlung erfolgte nur auf einem kleinen Bereich der Probe, so dass in der Röntgenbeugung immer auch Anteile der unbestrahlten Probe zu sehen sind. Um die Identifikation der durch die Bestrahlung erzeugten Effekte zu ermöglichen, wurde von den Messkurven zusätzlich das Spektrum eines unbestrahlten Teils der jeweiligen Probe abgezogen. Die jeweiligen Spektren wurden auf den Wert des Untergrunds nominiert, um auch Veränderungen der Peakhöhen erkennen zu können.

Im Vergleich zu einem unbestrahlten Teil der bei Raumtemperatur bestrahlten Probe zeigt sich in Abbildung 5.9 ein deutlich erhöhter Peak bei 


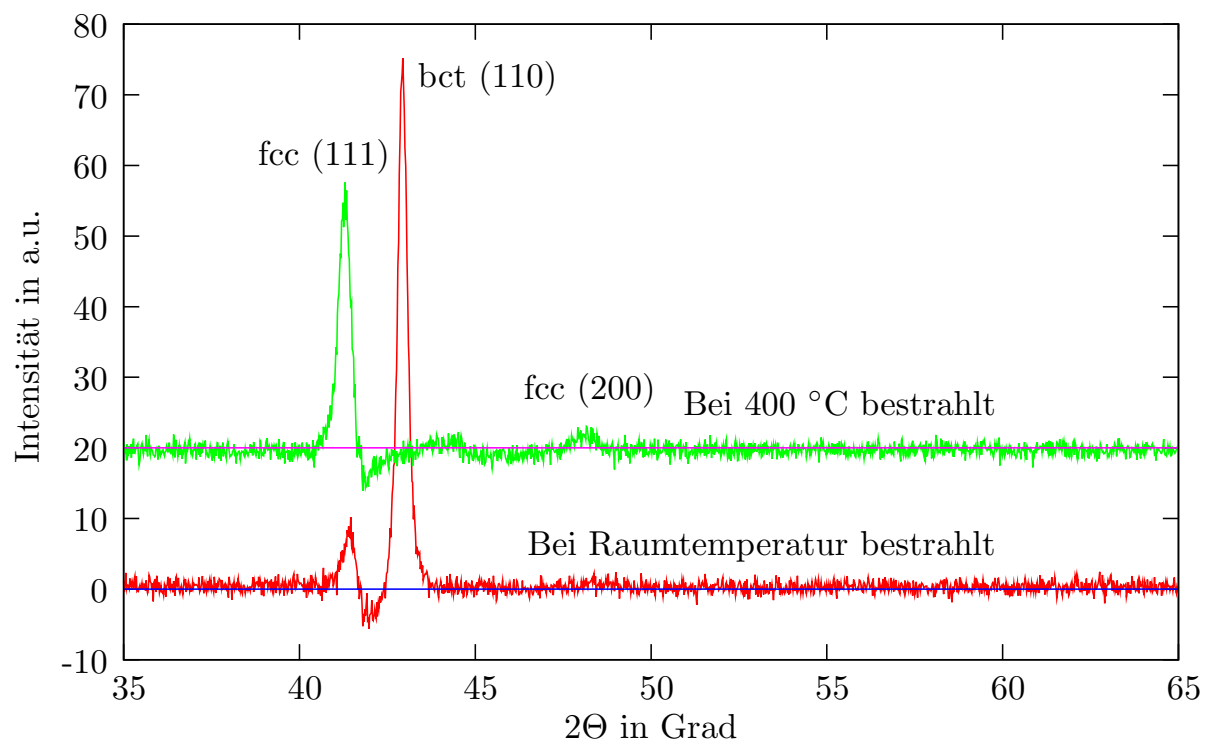

Abbildung 5.9: Differenzen zwischen den Intensitäten der bestrahlten und unbestrahlten Bereiche der bei Raumtemperatur und $400^{\circ} \mathrm{C}$ bestrahlten Proben.

$42.93^{\circ}$, der der bct-Struktur zugeordnet werden kann, und auf ein deutliches Anwachsen der Körner durch Rekristallisation hinweist. Die bei erhöhter Temperatur bestrahlte Probe in Abbildung 5.9 weist diesen bct-Peak nur sehr schwach auf. Hier ist im unbestrahlten Bereich der Probe deutlich ein Peak von $\alpha$-Eisen zu sehen, der zeigt, dass die Probe entmischt. Im bestrahlten Bereich ist dieser Reflex deutlich weniger ausgeprägt, stattdessen dominiert der fcc(111)-Peak. Die hierfür verantwortlichen Prozesse werden in Kapitel 6.3.1 diskutiert.

\section{$5.2 \quad$ Epitaktische Schichten}

Während es mittels gepulster Laserdeposition möglich ist, epitaktische Schichten bei Raumtemperatur auf $\mathrm{MgO}(100)$-Substrate zu wachsen, sind hierfür beim Elektronenstrahlverdampfen sehr hohe Substrattemperaturen notwendig. Während in den mittels PLD hergestellten Filmen daher Diffusion nur eine untergeordnete Rolle spielt, sind die Eigenschaften von bei hohen Temperaturen aufgedampften Filmen stark von thermisch aktivierten Relaxationsprozessen geprägt. 


\subsubsection{Mittels PLD hergestellte Schichten}

Im Rahmen einer Kooperation innerhalb des Schwerpunktprogramms 1239 der DFG wurden gemeinsam mit J. Buschbeck in der Arbeitsgruppe von S. Fähler am IFW Dresden Schichten mittels gepulster Laserdeposition auf $\mathrm{MgO}(100)$-Substraten hergestellt, und dabei der Spannungszustand der Filme und dessen Einfluss auf die Struktur untersucht. Hierbei wurde wie in Kapitel 3.2 dargestellt, in festen Zeitabständen von etwa einer Sekunde die Substratkrümmung bestimmt. Bei dieser Depositionsanlage war es nicht möglich, die Depositionsraten von Eisen und Palladium direkt zu messen, so dass diese aus der Schichtdicke und der Zusammensetzung der Schicht nachträglich bestimmt werden mussten. Hierfür wurden weitere Filme auf Siliziumsubstraten deponiert. Untersuchungen im REM (Abbildung 5.10) zeigen zunächst eine für PLD typische, relativ glatte Schicht mit einigen Tröpfchen (sog. Droplets) auf der Oberfläche der Schicht.

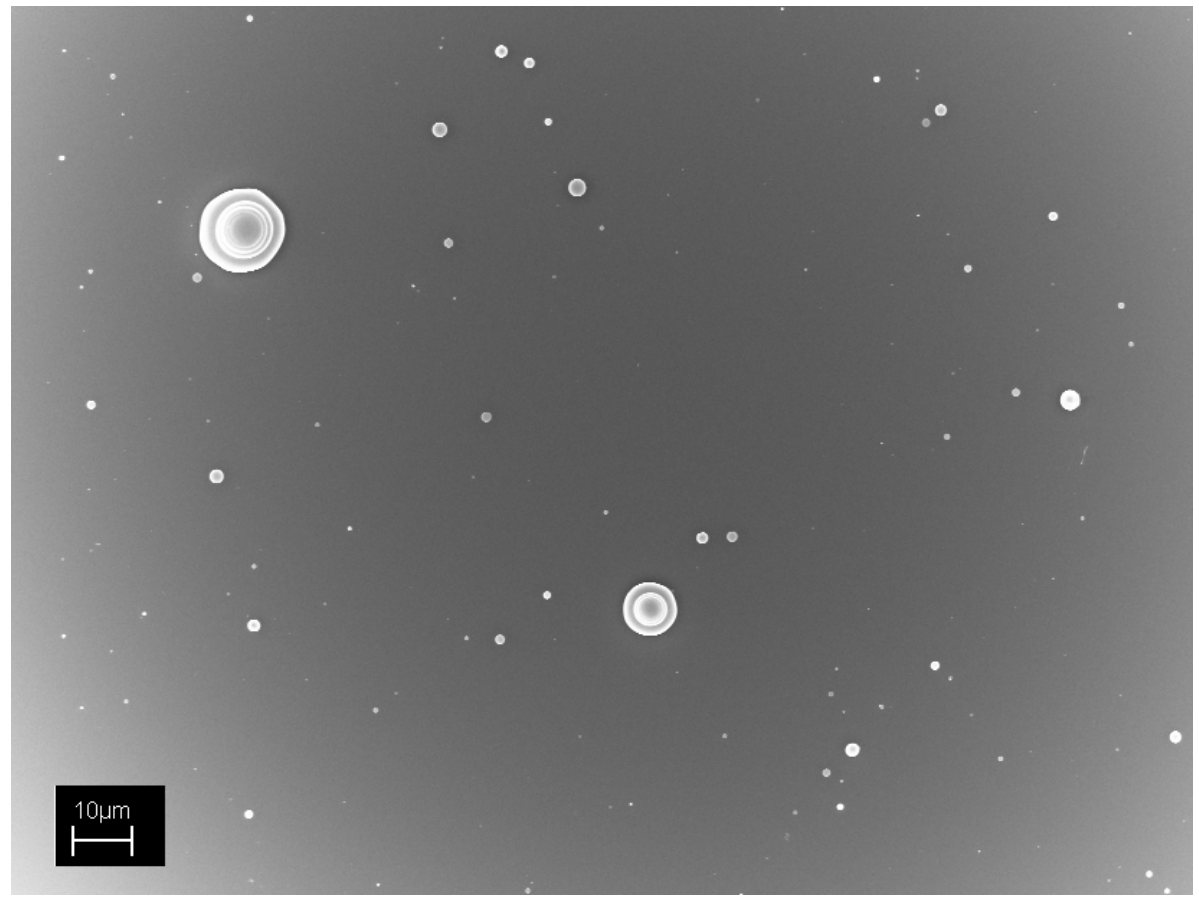

Abbildung 5.10: REM-Aufnahme einer mittels PLD hergestellten $\mathrm{Fe}_{70} \mathrm{Pd}_{30^{-}}$ Schicht mit Tröpfchen.

Obwohl die Kleinwinkel-Röntgenstreuung eine sehr gute Methode der Schichtdickenbestimmung darstellt, war es bei diesen Proben nicht möglich Oszillationen der Intensität zu messen, was auf eine inhomogene Schichtdicke 


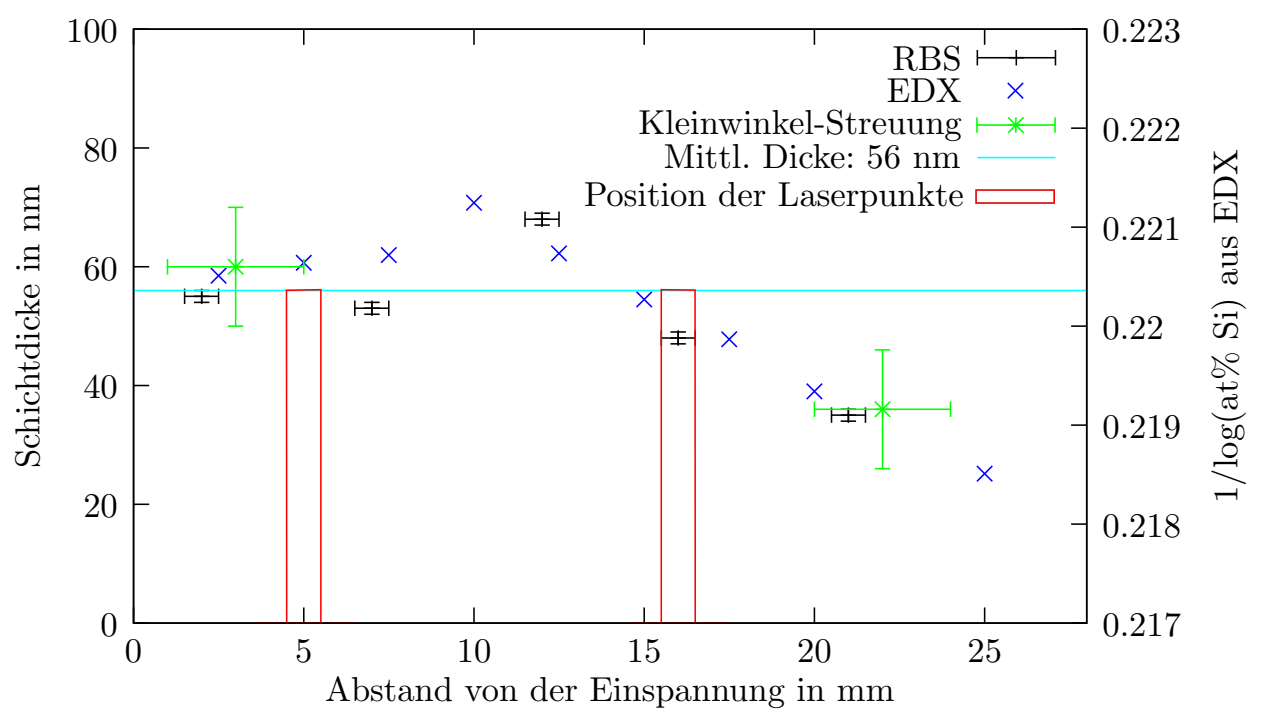

Abbildung 5.11: Bestimmung der Schichtdicke an einer mittels PLD hergestellten Probe.

hinweist. Um eine zerstörungsfreie, ortsaufgelöste Messung der Schichtdicke zu erhalten, wurde die Probe mit $\mathrm{He}^{2+}$-Ionen bestrahlt und die Energie der rückgestreuten Kerne untersucht. Die so gewonnenen RBS-Spektren lassen sich mit Spektren, die mit RUMP [88] simuliert wurden vergleichen, und durch Anpassung der Simulationsparameter (insbesondere der Schichtdicke) in Übereinstimmung bringen. Die laterale Ortsaufösung ist hierbei durch die Strahlbreite gegeben und liegt im Bereich von ca. $2 \mathrm{~mm}$.

Eine bessere Ortsauflösung kann mit Hilfe von EDX erreicht werden, wobei hier allerdings keine keine absoluten Werte für die Schichtdicke gewonnen werden können. Bei der Bestimmung der Schichtzusammensetzung mittels EDX wird bei dünnen Schichten auch immer ein gewisses Signal vom Substrat mit detektiert. Die Intensität dieses Signals hängt exponentiell von der Schichtdicke ab, da die entstehende Röntgenstrahlung den ganzen Film durchdringen muss, und dabei geschwächt wird. Nach diesen Untersuchungen wurde eine Probe in kleine Streifen zerteilt, an denen mittels Kleinwinkelstreuung die Schichtdicke grob bestimmt werden konnte. Die Ergebnisse der verschiedenen Messmethoden sowie die Positionen der beiden Laserstrahlen zur Messung der Substratkrümmung sind in Abbildung 5.11 dargestellt.

Aus diesem recht inhomogenen Verlauf der Schichtdicke ergibt sich, dass die Angabe der Schichtdicke zu einem bestimmten Zeitpunkt immer nur als mittlere Schichtdicke verstanden werden kann. Allerdings liegen die größten 


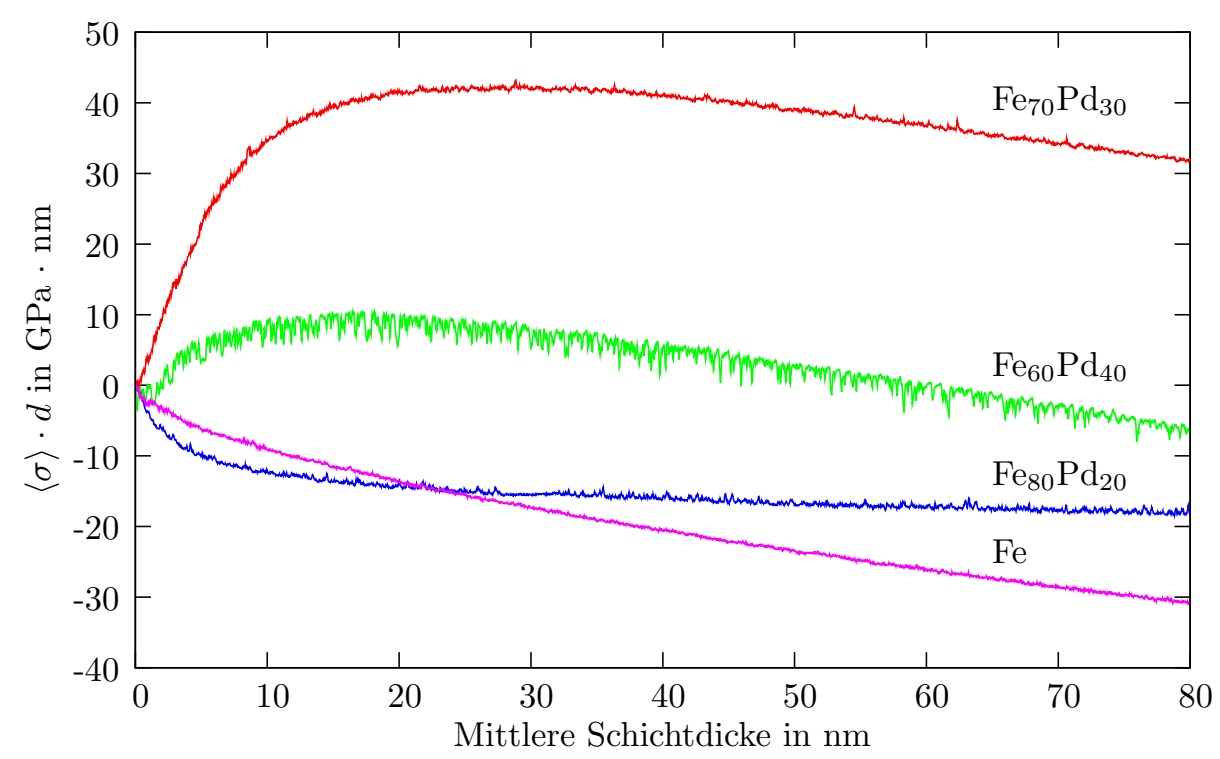

Abbildung 5.12: In-situ Substrat-Krümmungsmessungen während der Deposition von Eisen-Palladium-Schichten mit PLD auf MgO. Aus [103].

Abweichungen in dem Bereich, der außerhalb des zweiten Laserstrahls liegt. In dem Bereich zwischen den beiden Strahlen, in dem die Krümmung gemessen wird, sind die Abweichungen geringer.

Die gemessenen Spannungen (Abbildung 5.12) hängen sehr stark von der Zusammensetzung und der Struktur der jeweiligen Schicht ab. Die reine Eisenschicht, wie auch die Schicht mit $20 \%$ Palladiumanteil wachsen in einer bcc-Struktur auf [35] und weisen über den gesamten Bereich der Messung Druckspannungen auf, während die Schichten mit einem Palladiumgehalt von 30 und 40 Atomprozent in der fct-Struktur aufwachsen [35], und zunächst Zugspannungen und erst später Druckspannungen beobachtet werden. Die Eisenschicht weist über den gesamten beobachteten Bereich eine näherungsweise konstante Druckspannung von $-220 \pm 10 \mathrm{MPa}$ auf, während die $\mathrm{Fe}_{80} \mathrm{Pd}_{20}$-Schicht zunächst eine erhöhte Spannung zeigt, die für Schichtdicken von über $10 \mathrm{~nm}$ nur noch $-37 \pm 7 \mathrm{MPa}$ beträgt. Die beiden Schichten in der fcc Struktur weisen zu Beginn des Wachstums Zugspannungen von 4,64 \pm , $03 \mathrm{GPa}$ bei $30 \%$ Palladium bzw. 1,5 $\pm 0,05 \mathrm{GPa}$ bei $40 \%$ Palladium. In der späteren Phase des Wachstums, für Schichtdicken deutlich über $40 \mathrm{~nm}$, zeigen diese Schichten Druckspannungen von $-240 \pm 10 \mathrm{MPa}$ und $-300 \pm 10 \mathrm{MPa}$, und damit Werte, die Vergleichbar mit der reinen Eisenschicht sind. Eine Unterbrechung der Deposition für 10 Minuten bei einer der Messungen führte zu einer Änderung der Spannung um weniger als $10 \mathrm{MPa}$, 
so dass unter diesen Bedingungen signifikante Relaxationen der Probe ausgeschlossen werden können.

Aus einer $\mathrm{Fe}_{70} \mathrm{Pd}_{30}$-Schicht wurde mittels FIB eine TEM-Lamelle präpariert, in der in Abbildung 5.13 drei deutlich unterscheidbare Bereiche zu sehen sind: Ganz links befindet sich zunächst das MgO-Substrat, das eine klare Grenzfläche zum Film hat. Eine Fouriertransformation eines Ausschnitts des substratnahen Films zeigt zwei Hauptreflexe, die genau parallel zur Substratoberfläche liegen, sowie weitere schwächere Reflexe, die auf eine einkristalline fcc-Struktur hindeuten. Diese ca. $20 \mathrm{~nm}$ dicke Schicht weist einen fleckigen Kontrast auf, wie er typisch für verspannte TEM-Lamellen ist. Ab einer Entfernung von über $20 \mathrm{~nm}$ vom Substrat ist dieser Kontrast völlig verschwunden, allerdings ist hier eine (111)-Verzwillingung zu sehen, und auch die Fouriertransformation weist viele weitere Reflexe auf. Diese (111)-Verzwillingung wurde auch in Röntgenmessungen an einer Serie verschieden dicker Schichten bestätigt [35]: Bei $20 \mathrm{~nm}$ Schichtdicke ist nur der (200)-Reflex der Schicht zu sehen, was auf epitaktisches Wachstum hindeutet, mit zunehmender Schichtdicke bei 40 bis $250 \mathrm{~nm}$ bleibt der (200)-Reflex fast unverändert, während der (111)-Reflex deutlich anwächst.

Zusätzlich zu den in-situ Spannungsmessungen wurde an diesen Proben auch ex-situ die Verspannung bestimmt, indem in einem 4-Kreis Goniometer verschiedene Röntgenreflexe vermessen und hierfür jeweils die Gitterkonstante bestimmt wurde. Abbildung 5.14 zeigt dies für die beiden Schichten in der bcc-Struktur, die reine Eisenschicht und die $\mathrm{Fe}_{80} \mathrm{Pd}_{20}$-Schicht. Aus dem Unterschied zischen der Gitterkonstante in der Substratebene und der Gitterkonstante senkrecht zur Substratebene lässt sich mittels der in Kapitel 3.5 diskutierten $\sin ^{2} \Phi$-Methode [86] die Verzerrung $\varepsilon$ der Einheitszelle berechnen. Aus dieser kann im Rahmen der linearen Elastizitätstheorie die mechanische Spannung bestimmt werden, die diese Deformation bewirkt. Die beiden Größen sind über den Biaxialmodul $B$ verknüpft, der sich über den Elastizitätsmodul $E$ und die Querkontraktionszahl $\nu$ darstellen lässt:

$$
\sigma=B \cdot \varepsilon=\frac{E}{1-\nu} \cdot \varepsilon
$$

Verwendet man einen Elastizitätsmodul von $E=208 \mathrm{GPa}$ für reines Eisen [104] und $E=86 \mathrm{GPa}$ für $\mathrm{Fe}_{80} \mathrm{Pd}_{20}$ [16] und nimmt eine Querkontrationszahl von $\nu=1 / 3$ an, erhält man für den Eisenfilm eine Spannung von $\sigma_{\text {ip }}=880 \mathrm{MPa}$ und für den $\mathrm{Fe}_{80} \mathrm{Pd}_{20}$-Film $\sigma_{\text {ip }}=580 \mathrm{MPa}$. Dieses Ergebnis weicht deutlich von den in-situ bestimmten Werten ab, und wird in Kapitel 6.2.1 diskutiert. Für die anderen untersuchten Zusammensetzungen ist es im Prinzip zwar ebenfalls möglich, mit dieser Methode das Verhältnis der Achsen der Gitterzelle in der Schichtebene und senkrecht zur Schichtebene 


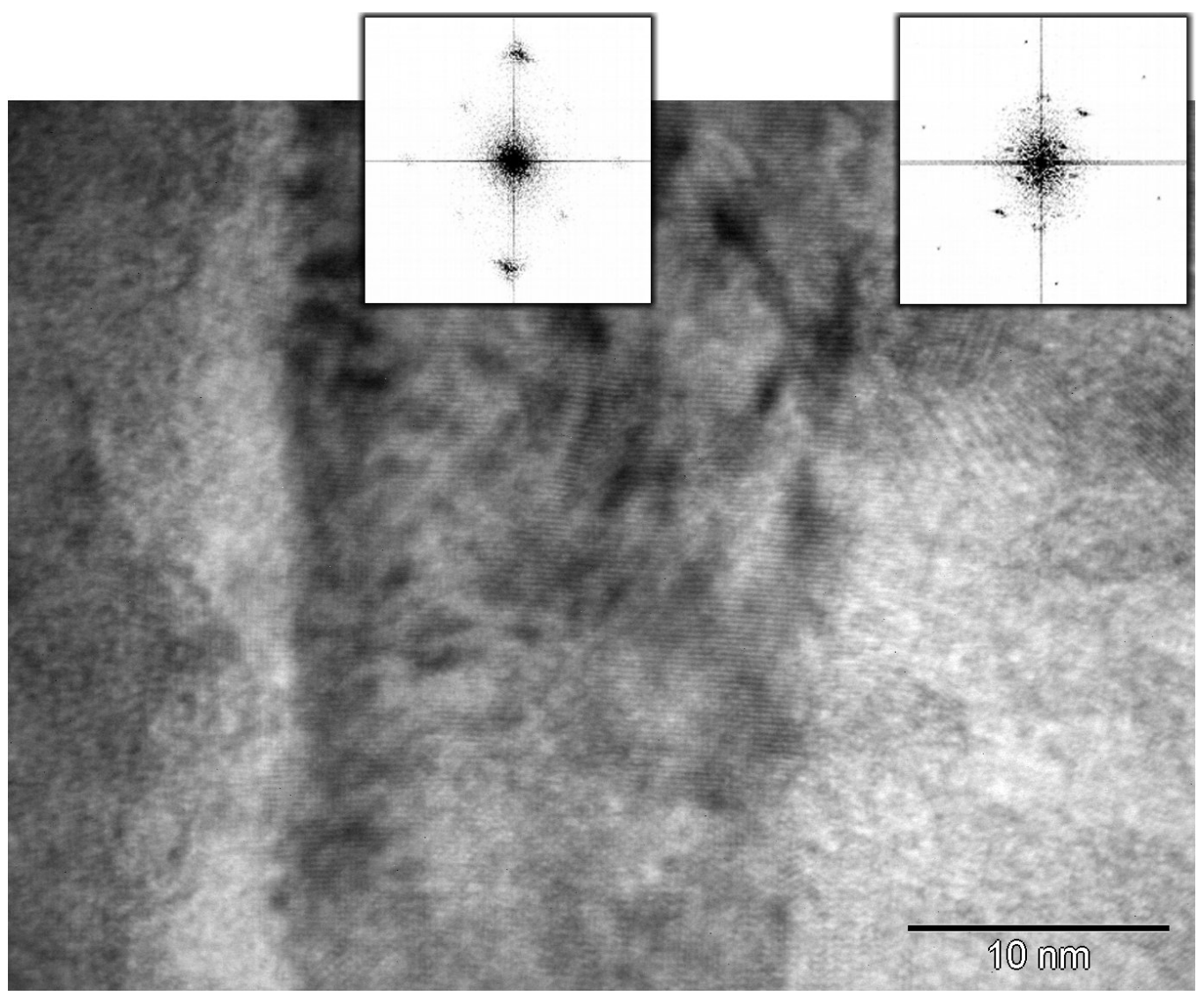

Abbildung 5.13: TEM-Aufnahme eines Querschnitts durch eine mit PLD abgeschiedenen $\mathrm{Fe}_{70} \mathrm{Pd}_{30}$-Schicht. Deutlich ist die Substrat-Film-Grenzfläche im linken Drittel des Bildes und eine weitere Grenzfläche im rechten Drittel des Bildes zu sehen. Die Insets zeigen eine Fourier-Transformation des Films im jeweiligen Gebiet. Aus [103] 


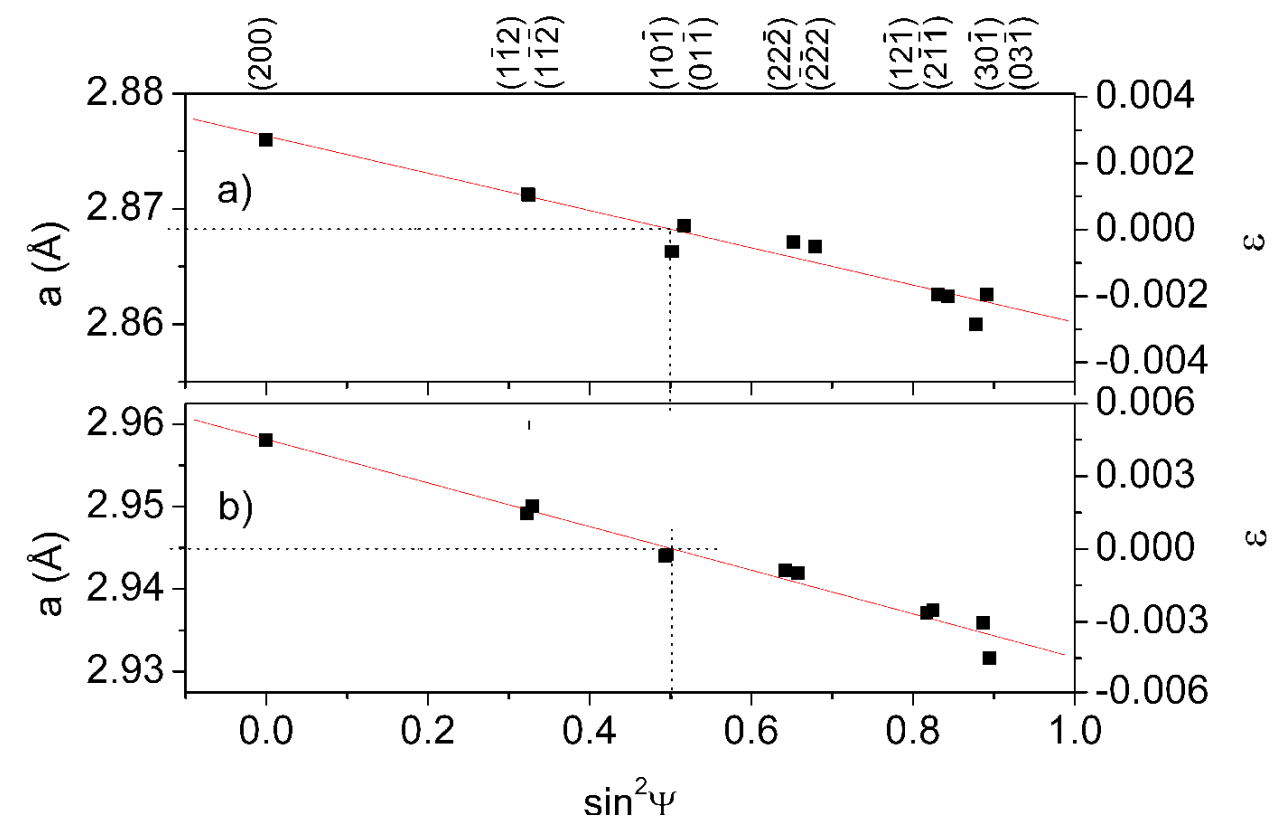

Abbildung 5.14: $\sin ^{2} \Psi$-Messungen an einer mittels PLD hergestellten Eisenschicht (a) und einer $\mathrm{Fe}_{80} \mathrm{Pd}_{20}$-Schicht (b). Aus [103].

$\mathrm{zu}$ bestimmen, diese liegen allerdings in einer tetragonal verzerrten Struktur vor [35], so dass hier aus der Verzerrung der Einheitszelle nicht auf die Spannung geschlossen werden kann.

\subsubsection{Aufgedampfte Schichten}

Epitaktisches Wachstum von Eisen-Palladium-Filmen auf MgO-Substraten ist beim Aufdampfen ab einer Substrattemperatur von $500^{\circ} \mathrm{C}$ möglich, Bei Temperaturen unter $690^{\circ} \mathrm{C}$ entmischen diese Schichten allerdings zumindest teilweise [34, 105]. Abbildung 5.15 zeigt eine hochauflösende TEM-Aufnahme der Grenzschicht, an der zu erkennen ist, dass sich die Kristallstruktur des Substrats in den Film hinein fortsetzt. Im Rahmen der vorliegenden Arbeit wurden insbesondere die Spannungen bei solchen Depositionstemperaturen untersucht, bei denen die Filme nicht entmischen. Im folgenden werden die Spannungen von drei $\mathrm{Fe}_{70} \mathrm{Pd}_{30}$-Filmen, die bei $630^{\circ} \mathrm{C}, 690^{\circ} \mathrm{C}$ und $750^{\circ} \mathrm{C}$ hergestellt wurden, näher beschrieben.

Direkt vor dem Aufdampfen, während die Verdampfer hochgefahren werden und direkt nach dem Aufdampfen, bis die Verdampfer abgekühlt sind, muss der Substrat-Shutter geschlossen werden, um ein unkontrolliertes Be- 


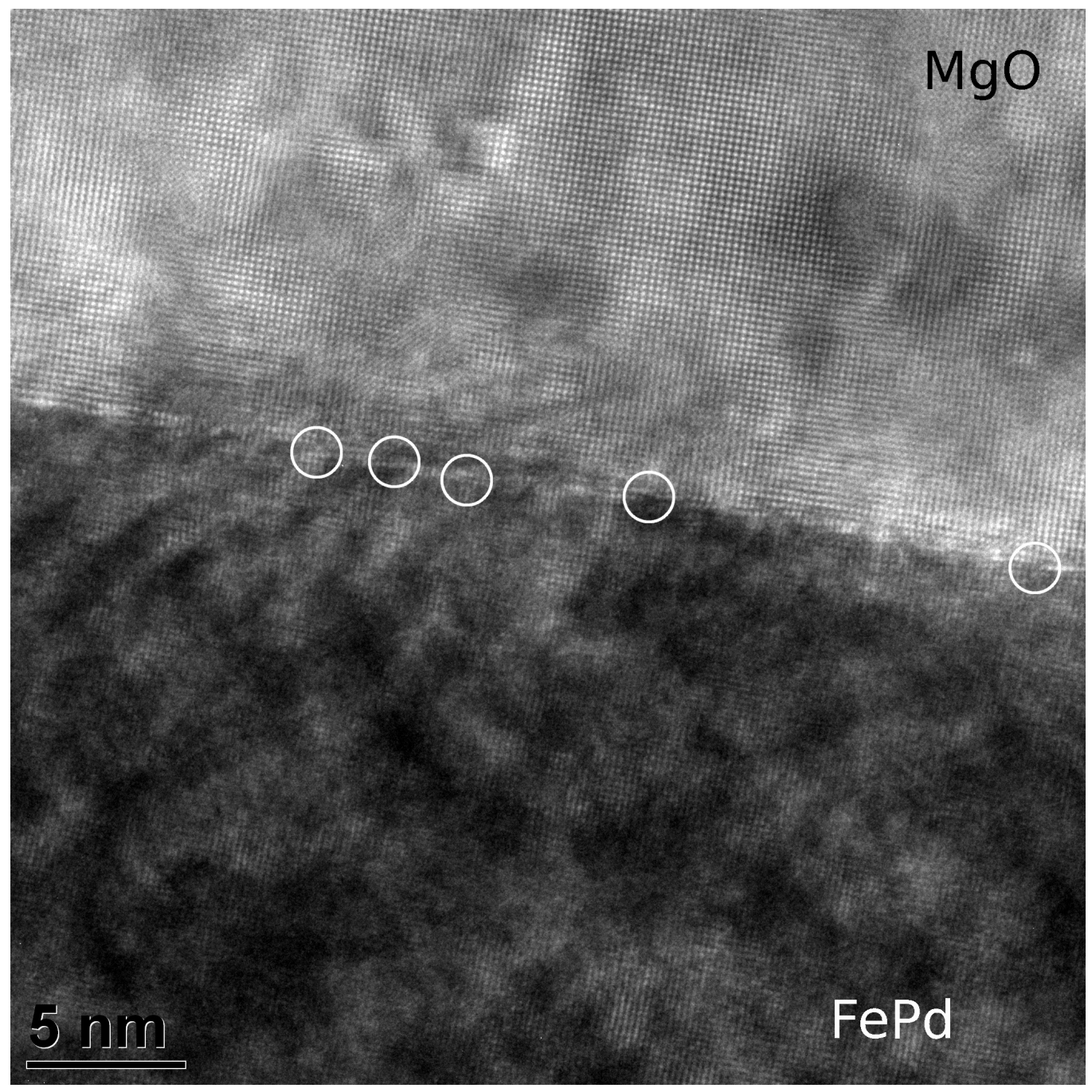

Abbildung 5.15: HRTEM-Aufnahme der Grenzschicht zwischen Film und Substrat. Die weißen Kreise markieren Versetzungen. 


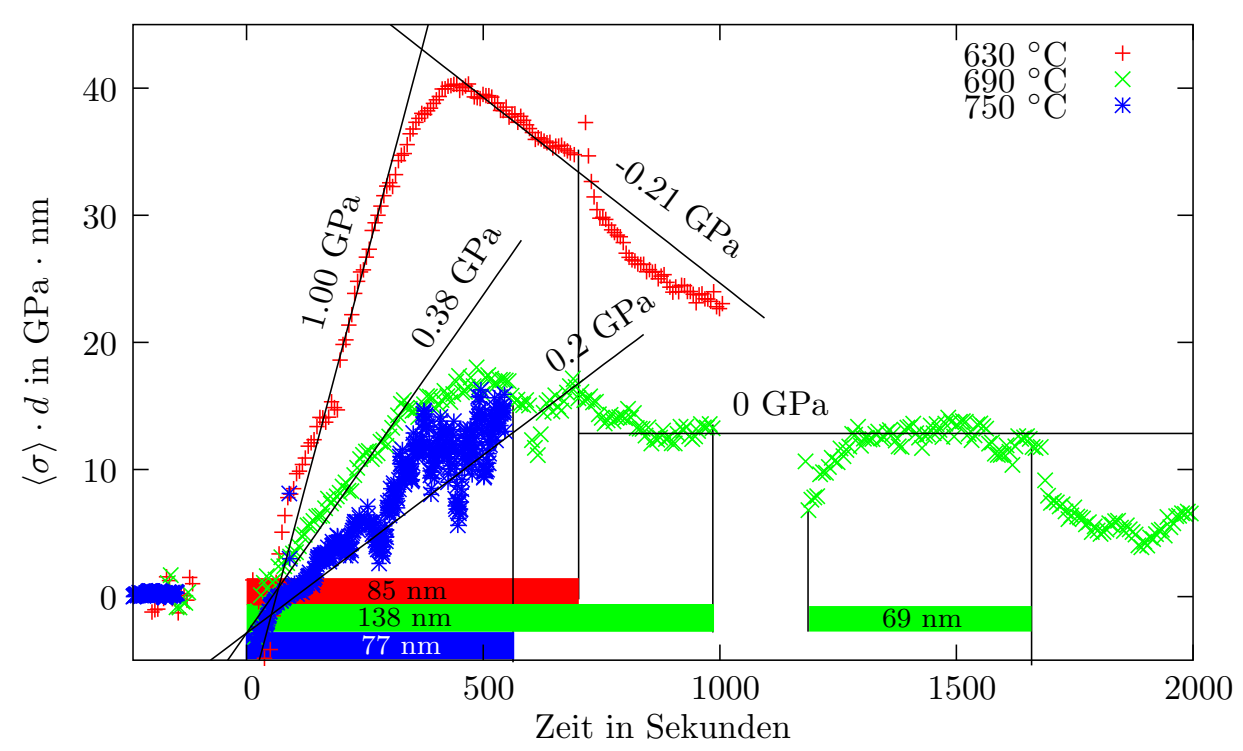

Abbildung 5.16: In-situ Substratkrümmungs-Messung während und nach dem Aufdampfen vom $\mathrm{Fe}_{70} \mathrm{Pd}_{30}$-Filmen bei verschiedenen Temperaturen. Die Zeiträume, in denen Material deponiert wurde, sind durch farbige Balken markiert. Die Messung bei $630^{\circ} \mathrm{C}$ stammt aus der Diplomarbeit von Lisa Kühnemund [105].

dampfen des Substrats zu vermeiden. In dieser Zeit ist es nicht möglich, die Substratkrümmung zu vermessen, da der Shutter auch die Laserstrahlen unterbricht. Darüber hinaus tritt immer nach dem Öffnen des Substrat-Shutters und während der darauf folgenden 120 Sekunden eine charakteristische Änderung der Verkippung des Substrats auf, die unabhängig davon ist, ob ein Film auf das Substrat deponiert wird oder nicht. Diese Verkippung wird durch die Änderung der Temperatur des Heizersystems verursacht: Beim Öffnen des Shutters direkt vor dem Substrat erhöht sich der Leistungsbedarf um die Temperatur zu halten, da nun weniger Strahlung von dem Shutter reflektiert wird. Daher sinkt zu Beginn der Deposition die Temperatur um einige Grad, und verkippt dadurch den kompletten Substrathalter. Temperaturmessungen mit einem Thermoelement direkt auf dem Substrat zeigen, dass nach ca. 2 Minuten (was bei einer Depositionsrate von $1.1 \AA$ pro Sekunde $15 \mathrm{~nm}$ Filmdicke entspricht) das thermische Gleichgewicht wieder erreicht wird. Daher wurden alle Daten aus diesem Zeitraum bei den Auswertungen und in Abbildung 5.16 nicht berücksichtigt.

Für den bei $630^{\circ} \mathrm{C}$ gewachsenen Film [105], der in Abbildung 5.16 rot dargestellt ist, beobachtet man eine Zugspannung von 1, $00 \pm 0,15 \mathrm{GPa}$ bis 
zu einer Filmdicke von $50 \mathrm{~nm}$, wo die maximale Krümmung auftritt. Nach dieser Dicke, ist die Steigung negativ (-0,21 \pm 0,15 GPa), was entweder als Druckspannung in der neu aufwachsenden Schicht oder als relaxieren der Zugspannung in den tiefer liegenden Schichten des Films verstanden werden kann. Nach dem Ende der Deposition nimmt die Krümmung exponentiell weiter ab.

Der in Abbildung 5.16 grün dargestellte bei $690^{\circ} \mathrm{C}$ deponierte Film wurde in zwei Schritten hergestellt: Zunächst wurde auf das Substrat ein Film mit einer Dicke von $138 \mathrm{~nm}$ abgeschieden. Danach wurde der Shutter unter dem Substrat geschlossen und das Material in den Verdampfern wurde erneut vollständig aufgeschmolzen. Dies ist bei notwendig, da während der Deposition bei niedrigen Verdampfungsraten nicht das gesamte Material vom Elektronenstrahl flüssig gehalten wird. So könnte es dazu kommen, dass sich tiefe Trichter im zu verdampfenden Material bilden, die die Abdampfcharakteristik und damit die Schichtzusammensetzung beeinflussen würden. Anschließend wurden weitere $69 \mathrm{~nm}$ deponiert.

Für diesen Film misst man eine Zugspannung von 0, 38 $\pm 0,05 \mathrm{GPa}$ während der ersten $40 \mathrm{~nm}$ des Filmwachstums, die maximale Krümmung des Substrats wird bei einer Dicke von $60 \mathrm{~nm}$ erreicht. Dieses Maximum ist allerdings deutlich weniger ausgeprägt als bei der bei niedrigerer Temperatur hergestellten Schicht. Während der zweiten Depositionsphase erhält man zunächst einen Anstieg der Krümmung während der ersten $20 \mathrm{~nm}$, danach ist die Krümmung des Substrats konstant und nahezu identisch mit dem Niveau vor der Unterbrechung. Die neu gewachsenen Schichten des Films ändern nun die Substratkrümmung nicht weiter, sie wachsen also spannungsfrei auf. Nach dem Ende dieser zweiten Depositionsphase ist eine weitere Abnahme der Substratkrümmung zu beobachten, die eine vergleichbare Zeitkonstante und Größe aufweist wie die Krümmungsänderung zu Beginn der zweiten Depositionsphase.

Der bei $750^{\circ} \mathrm{C}$ gewachsene, in Abbildung 5.16 blau dargestellte Film zeigt ein drastisch reduziertes Signal-Rausch-Verhältnis, da das von der Substratheizung emittierte Licht bei dieser Temperatur sehr viel intensiver ist. Dennoch kann die Spannung zu Beginn der Deposition zu etwa 0,2 GPa bestimmt werden, was sich gegen Ende des Filmwachstums etwas zu verringern scheint.

Betrachtet man die beim Wachstum des bei $690^{\circ} \mathrm{C}$ deponierten Films aufgezeichnete Intensität des reflektierten Laserstrahls in Abbildung 5.17, so kann man zu Beginn einen deutlichen Anstieg erkennen, der auf die mit zunehmender Schichtdicke zunehmende Reflektivität zurückzuführen ist. Bei höheren Schichtdicken ist ein Rückgang zu beobachten, der darauf hindeutet, dass sich die Rauigkeit der Schicht erhöht. Während der Depositionspause verändert sich die Intensität nur wenig, ab 1500 Sekunden nach Beginn der 


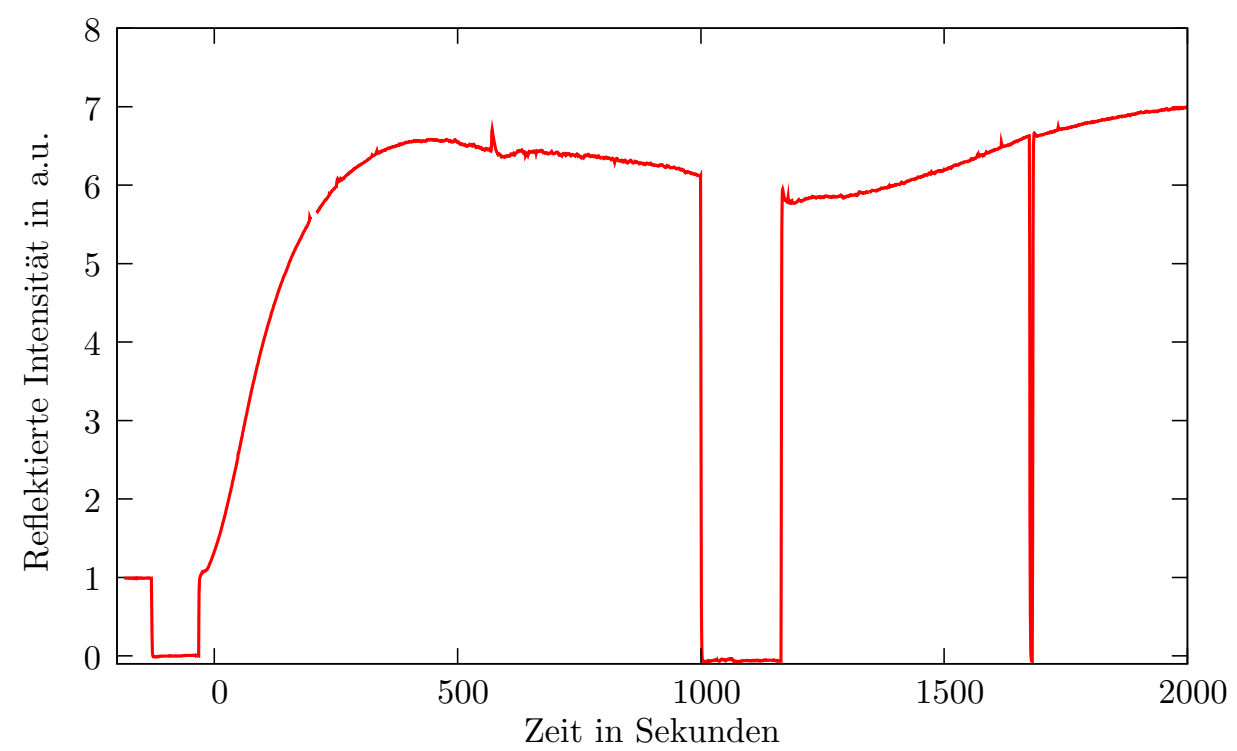

Abbildung 5.17: Intensität des reflektierten Laserstrahls während und nach der Deposition des bei $690^{\circ} \mathrm{C}$ hergestellten Films. Immer dann, wenn die Intensität auf Null fällt, war der Shutter geschlossen: Vor der Deposition, in der Pause zwischen der ersten und der zweiten Deposition, und noch einmal sehr kurz am Ende der zweiten Deposition

Deposition steigt das Signal dagegen wieder an, und setzt dies auch stetig fort, nachdem die Deposition abgeschlossen ist. Dies deutet auf ein Glätten der Schicht hin, und deckt sich mit Untersuchungen der Topologie von lange getemperten Filmen, in denen ebenfalls sehr glatte Oberflächen gefunden werden (siehe Abbildung 5.18).

Auch bei längerem Tempern bleibt die Gitterstruktur, wie sie sich beim Wachstum eingestellt hat, trotz der deutlichen Fehlpassung zwischen dem Film und dem Substrat erhalten. Ein für 20 Stunden bei $900{ }^{\circ} \mathrm{C}$ Grad getemperter Film zeigt in Abbildung 5.18 Strukturen, die genau parallel zu den [100]-Richtungen des Substrats verlaufen. Die Ecken zwischen diesen rechtwinklig zueinander verlaufenden Stufen sind allerdings teilweise um $45^{\circ}$ abgeschrägt. Diese Facettierung lässt sich dadurch erklären, dass bei einem $90^{\circ}$-Winkel das vorderste Atom nur extrem wenige Bindungspartnern hat, was eine energetisch sehr ungünstige Konfiguration darstellt. 


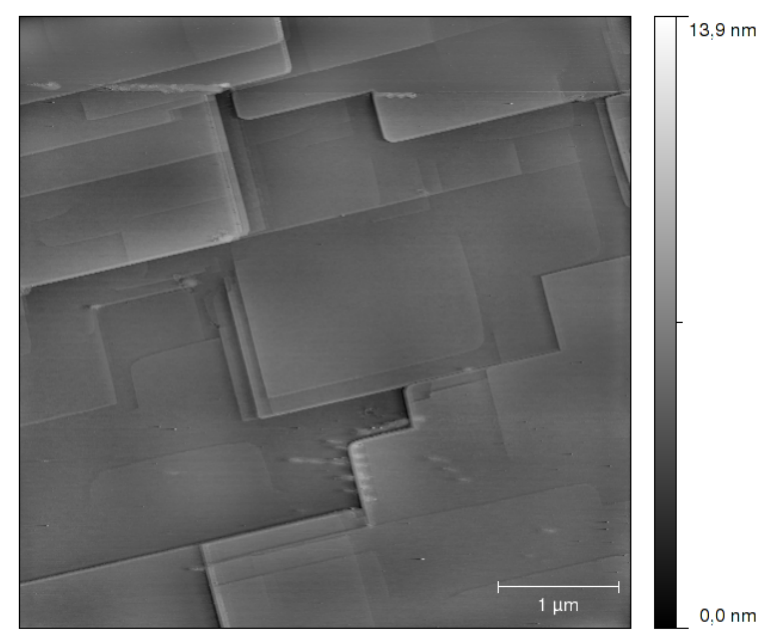

\begin{abstract}
Abbildung 5.18: STM-Topographie eines bei $900^{\circ} \mathrm{C}$ für 20 Stunden getemperten, $230 \mathrm{~nm}$ dicken $\mathrm{Fe}_{70} \mathrm{Pd}_{30}$ Films auf $\mathrm{MgO}$. Die sehr glatte Struktur wird nur von wenigen Stufen unterbrochen, die parallel zu den Substratkanten (und damit den [100]-Richtungen des Substrats) verlaufen.
\end{abstract}

\title{
5.3 Molekulardynamik-Simulationen
}

Der Einfluss von Defekten auf die Spannungsrelaxation in den Schichten kann mit Molekulardynamik-Simulationen detailliert verfolgt werden. Hierfür wurden, wie in Kapitel 4 beschrieben, verschiedene Zellen präpariert, die einen unendlich ausgedehnten Film mit einer freien Oberfläche wiedergeben, der epitaktisch auf einem harten Substrat aufliegt. Dieser Film wird nun mit einer Rate von $10^{-7} \mathrm{fs}^{-1}$ gedehnt, in dem die $x$ - und $y$-Koordinate reskaliert werden. In den beiden untersten Atomlagen, die das Substrat darstellen, werden dabei alle Atome fixiert, so dass sie sich nicht bewegen können, während der Rest des Films, insbesondere die Oberfläche, unter dem Einfluss dieser Dehnung relaxieren kann. Jede Momentaufnahme der Simulation kann so als Beispiel mit einer Gitterfehlpassung zwischen Schicht und Substrat verstanden werden. Um den Einfluss der in den Experimenten beobachteten Eisenausscheidungen in Filmen, die ihre Spannungen über Partialversetzungen abbauen $[34,105]$ zu untersuchen, werden daher sowohl Zellen ohne als auch mit Eisenausscheidungen untersucht.

Betrachtet man nun zunächst das Relief der freien Oberfläche einer Simulationszelle ohne Eisenausscheidungen in Abbildung 5.19, so beobachtet man oberhalb einer kritischen Dehnung von 4,48\% streifenförmige Modulationen 
der Oberfläche. Um die hierbei ablaufenden Prozesse im Volumen der Simulationszelle beobachten zu können, ist es notwendig alle Atome auszublenden, die nicht von Interesse sind. Hierzu ist es günstig, den in Kapitel 4.3 beschriebenen Zentral-Symmetrie-Parameter $c$ zu betrachten, der angibt, wie weit die Konfiguration der nächsten Nachbaratome eines Atoms von einer punktsymmetrischen Anordnung abweicht. Man kann zeigen, dass dieser Parameter für einen Stapelfehler in einem perfekten fcc-Kristall einen Wert von $c=0,0416$ annimmt, während thermische Fluktuationen unterhalb der Schmelztemperatur (bei der nach der Lindemann-Gilvarry-Regel die Schwingungsamplituden der Atome ungefähr $12 \%$ des Nächsten-Nachbar-Abstandes betragen [106]) zu einem Wert von unter 0,01 führen [101]. Für die Darstellung wird nun von der Software AtomEye [100] für jedes Atom dieser Wert $c$ berechnet, so dass es möglich ist, in jedem Zeitschritt nur diejenigen Atome darzustellen, deren Zentralsymmetrie-Parameter oberhalb eines Schwellwerts liegt, um thermische Fluktuationen auszublenden. In den hier gezeigten Bildern werden nur solche Atome dargestellt, bei denen der Zentralsymmetrie-Parameter im Intervall $0.014<c<0.4$ liegt. Der Wert von $c$ ist darüberhinaus farbcodiert, die blau dargestellten Atome haben hierbei den kleinsten Wert, die rot dargestellten Atome den größten. Die Farbskala und das Abschneidekriterium wurden so gewählt, dass Stapelfehler deutlich in blau erscheinen, während die Oberflächen, an denen die Symmetrie natürlich viel stärker gebrochen ist, in gelb und rot erscheinen. Im Inneren der Simulationszelle ohne Eisenausscheidungen sind für kleine Dehnungen nur punktuelle Fluktuationen des Zentralsymmetrie-Parameters zu erkennen. Die zu den in den Abbildungen 5.19 und 5.21 bis 5.23 gezeigten Zeitpunkten gehörenden Dehnungen lassen sich bei der Dehnungsrate von $10^{-7} \mathrm{fs}^{-1}$ leicht ablesen, bei $t=454.5$ ps beträgt die Dehnung zum Beispiel 4.545\%.

$\mathrm{Ab}$ einer Dehnung von 4,48 \% ist in Abbildung $5.21 \mathrm{zu}$ erkennen, wie sich von der freien Oberfläche her zwei Flächen in das Volumen der Zelle ausbreiten, an denen $c$ deutlich über dem Schwellwert liegt, und die somit blau dargestellt sind. Diese flächenartigen Defekte lassen sich als $a / 6\langle 112\rangle$ Shockley-Partialversetzungen identifizieren, die über die $\{111\}$-Ebenen laufen. Im weiteren Verlauf der Simulation laufen dann weitere Partialversetzungen über benachbarte $\{111\}$-Ebenen. Dies überführt die Stapelfolge der $\{111\}$-Ebenen in eine zur ursprünglichen Konfiguration spiegelsymmetrische Anordnung: Die ungestörte Stapelfolge ABCABC wird nun von einem Bereich mit der Stapelfolge $\mathrm{ABCAB}|\mathrm{ACBACBA}| \mathrm{BCABC}$ unterbrochen. Innerhalb des Bereichs zwischen den beiden Ebenen ist das Gitter nun wieder frei von Stapelfehlern, und die Grenzen dieses Bereichs sind gerade die Zwillingsgrenzen, wie sie auch bei einer martensitischen Umwandlung auftreten [31]. Die über die Zwillingsgrenzen gleitenden Partialversetzungen ordnen somit 


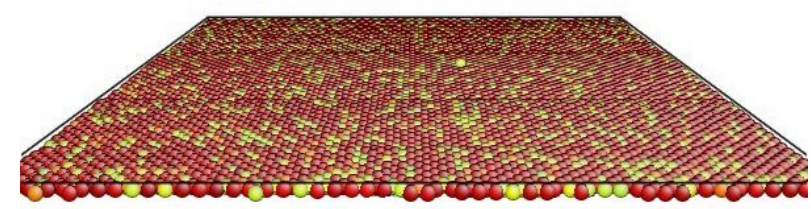

(a) $t=35,504 \mathrm{ps}$

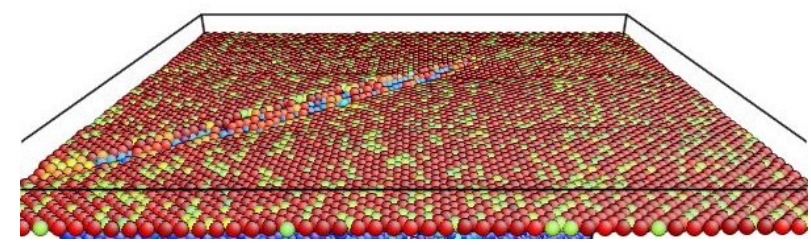

(b) $t=474,0 \mathrm{ps}$

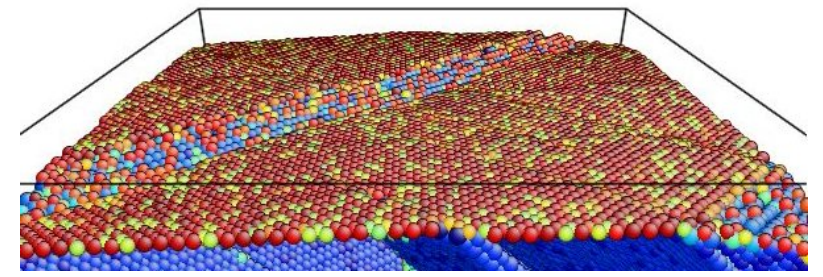

(c) $t=494,5 \mathrm{ps}$

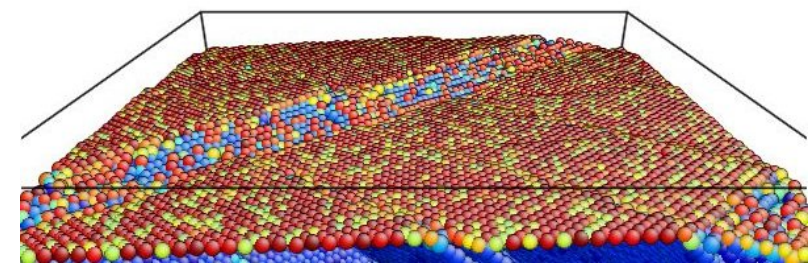

(d) $t=519,0 \mathrm{ps}$

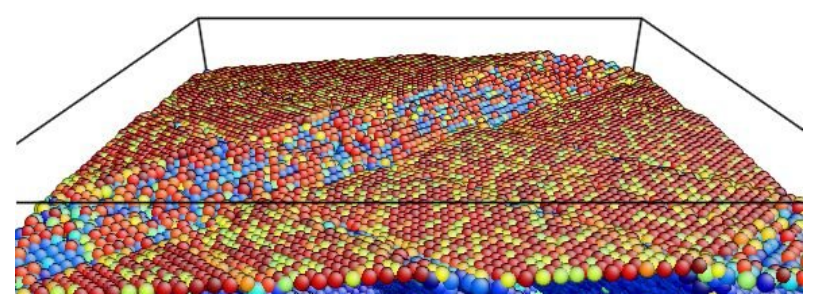

(e) $t=690,5 \mathrm{ps}$

Abbildung 5.19: Oberfläche der Simulationszelle zu verschiedenen Zeitpunkten, während sie gedehnt wird. Die zunächst atomar glatte Fläche weist ab einer Dehnung von 4,48 \% zunächst eine Stufe auf, mit wachsender Dehnung wird diese ausgeprägter, und weitere Stufen treten auf. 

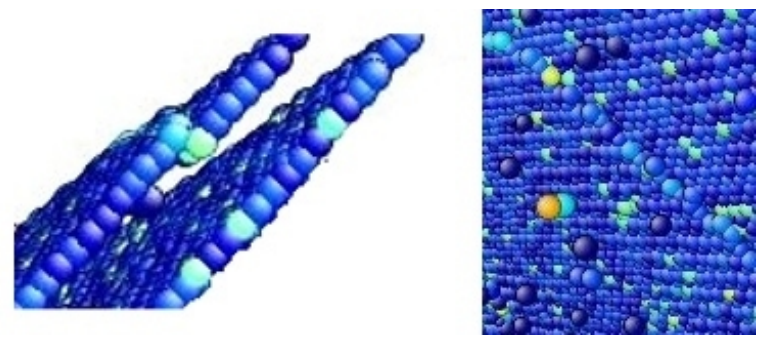

Abbildung 5.20: Partialversetzung, links: Seitenansicht, rechts: Ansicht senkrecht zur Ausbreitungsebene.

die nächsten Atomlagen so an, dass sie nun nicht mehr zur der den Zwilling umgebenden Ursprungsstruktur gehören, sondern sich an die Struktur des Zwillings anpassen, so dass dieser dadurch breiter wird. Abbildung 5.20 zeigt eine Detailansicht einer solchen Partialversetzung. Da diese über die Zwillingsebenen gleitenden Partialversetzungen keine neuen Grenzflächen erzeugen und somit auch keine Grenzfächenenergie zu ihrer Bildung aufgewendet werden muss, ist leicht vorstellbar, dass sie nun lawinenartig durch den Kristall laufen und dabei die elastische Verspannung abbauen. Durch den Vergleich der Bilder bei 464.5 ps und 468.5 ps kann man abschätzen, dass sich die Partialversetzung in diesen 4 ps etwa $6.4 \mathrm{~nm}$ weit bewegt hat, was einer Geschwindigkeit von etwa 1600 Metern pro Sekunde entspricht, und damit etwas unter der Schallgeschwindigkeit in FePt liegt [107].

Das schon beobachtete Oberflächenrelief lässt sich nun auch leicht verstehen: Die Grenzen der Oberflächenbereiche mit verschiedenen Oberflächennormalen sind genau die Zwillingsgrenzen. Es ergibt sich ein Streifenmuster wie man es auch aus Topographie-Untersuchungen martensitischer Proben erwartet.

Die Simulationszelle mit Eisenausscheidungen weist ein sehr ähnliches Verhalten auf: Auch hier bilden sich zunächst Paare von $a / 6\langle 112\rangle$-ShockleyPartialversetzungen die über die $\{111\}$-Ebenen laufen. Damit bilden sich wieder Zwillinge, die dann durch weitere Partialversetzungen, die über die Zwillingsgrenzen laufen, in ihrer Dicke wachsen. Die Zwillinge bilden sich hier allerdings nicht nur von der freien Oberfläche aus, sondern nukleieren auch an den Eisenausscheidungen. Zusätzlich zum Wachstum der Zwillinge ist hier ein weiterer Effekt zu beobachten: An der in Abbildung 5.22 grün markierten Ausscheidung wird ein Zwilling gepinnt, so dass er nicht in seiner Dicke wachsen kann. Stattdessen bildet sich zwei Pikosekunden später ganz in der Nähe ein weiterer Zwilling, der die Eisenausscheidung nicht schneidet und dann rasch in seiner Dicke anwächst. Fünf weitere Pikosekunden später 


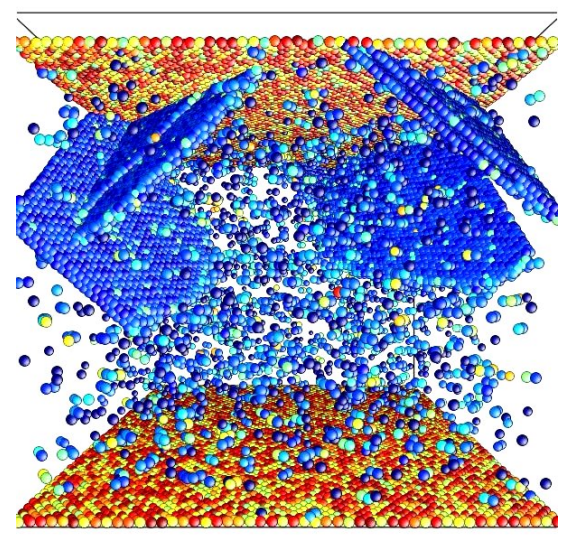

(a) $t=454.5 \mathrm{ps}$

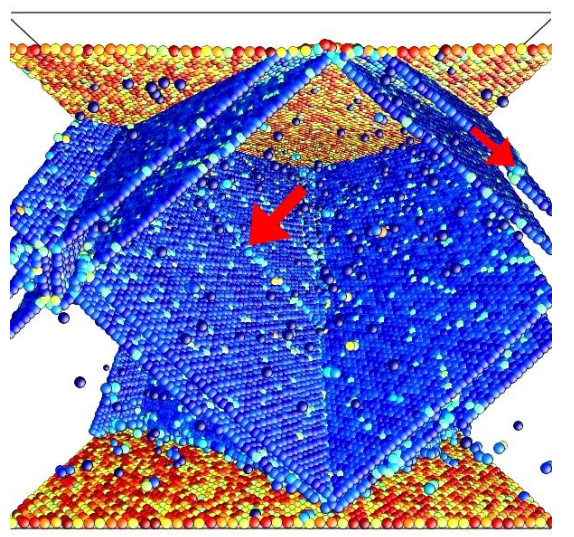

(c) $t=464.5 \mathrm{ps}$

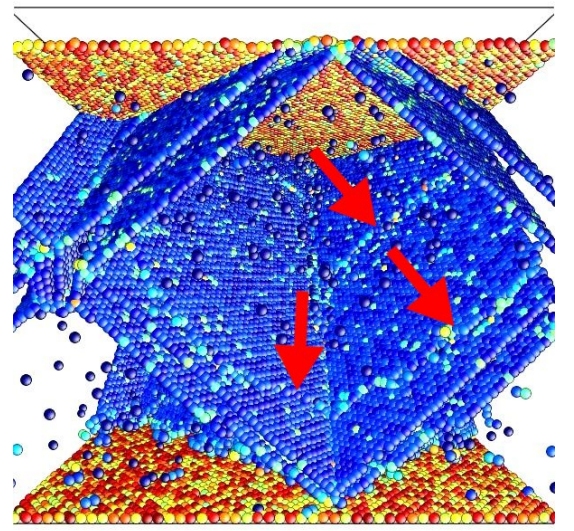

(e) $t=476.0 \mathrm{ps}$

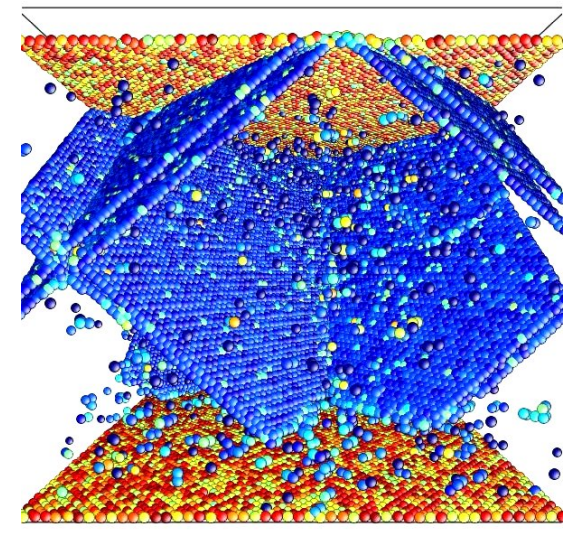

(b) $t=458.0 \mathrm{ps}$

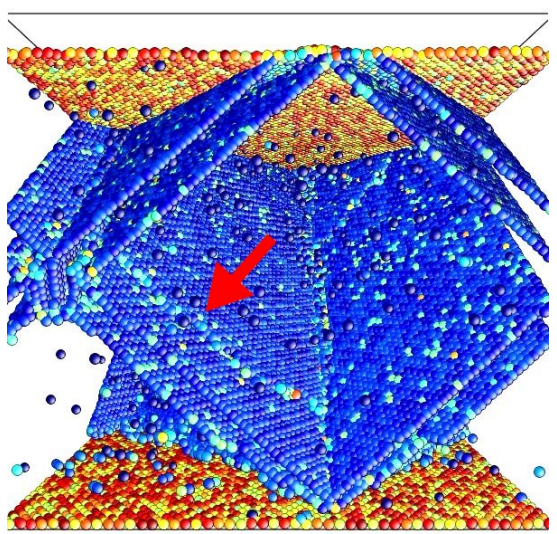

(d) $t=468.5 \mathrm{ps}$

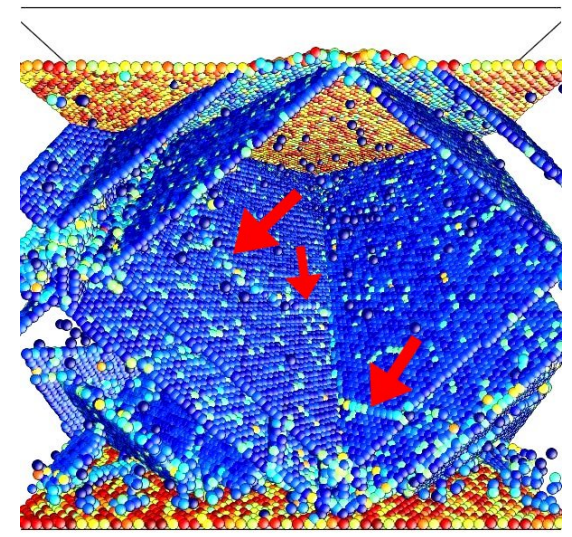

(f) $t=670.5 \mathrm{ps}$

Abbildung 5.21: Zelle ohne Ausscheidungen: Oben die freie Oberfläche, unten das Substrat. Die Zwillinge wachsen zunächst von der Oberfläche aus in die Schicht hinein, später wandern zusätzliche Partialversetzungen über die Grenzflächen und verbreitern damit die Zwillinge. 


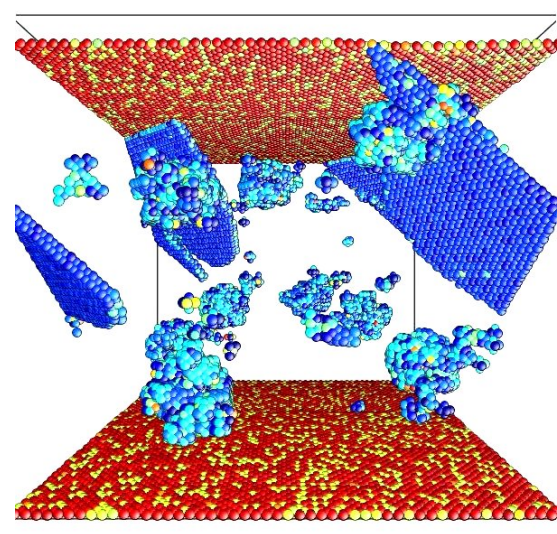

(a) $t=473.5 \mathrm{ps}$

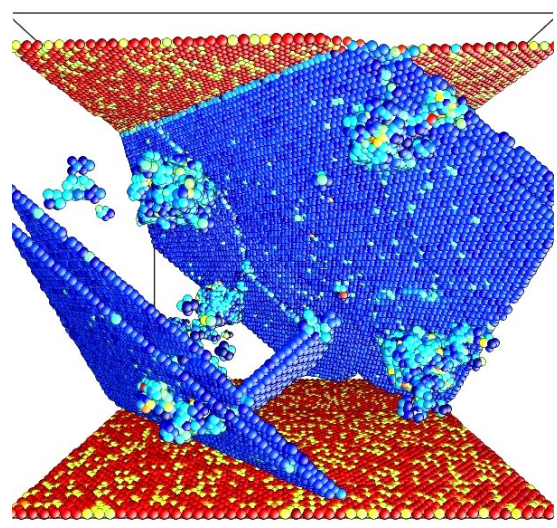

(c) $t=480.0 \mathrm{ps}$

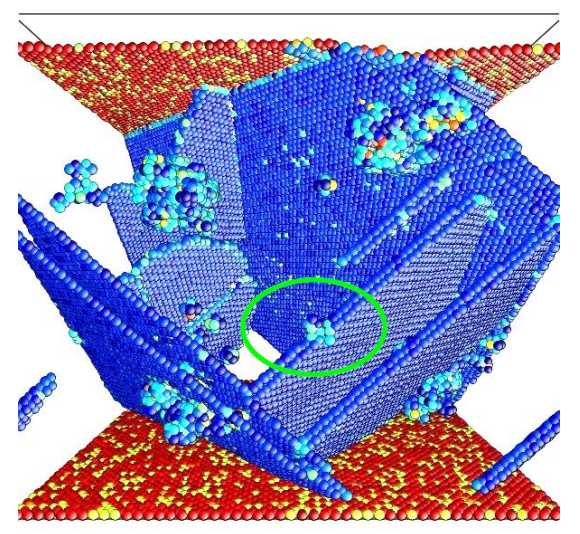

(e) $t=485.0 \mathrm{ps}$

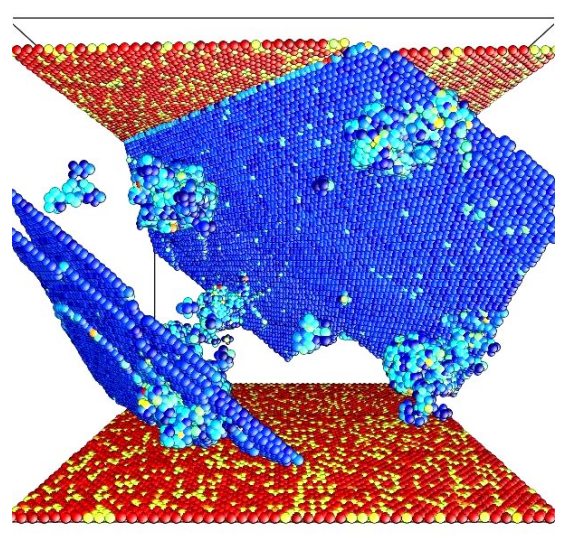

(b) $t=478.0 \mathrm{ps}$

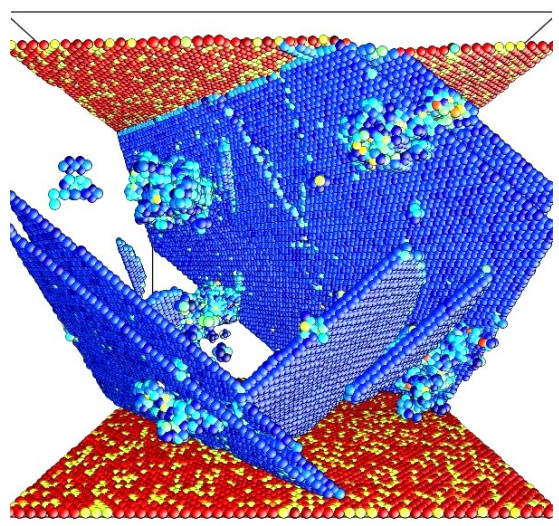

(d) $t=483.0 \mathrm{ps}$

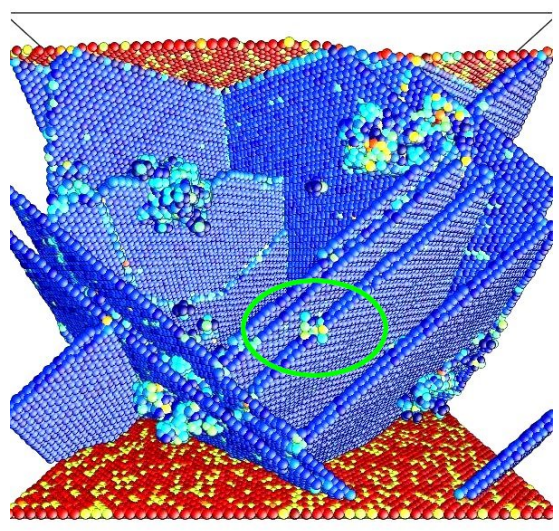

(f) $t=488.5 \mathrm{ps}$

Abbildung 5.22: Zelle mit Eisenausscheidungen: Oben die freie Oberfläche, unten das Substrat. Zwillinge bilden sich sowohl an der Oberfläche als auch an den Eisenausscheidungen. Im Vordergrund (grün markiert) wird eine Grenzfläche an einer Eisenausscheidungen gepinnt und wächst nicht in die Dicke, sondern es bildet sich ein neuer Zwilling in der Nähe. 


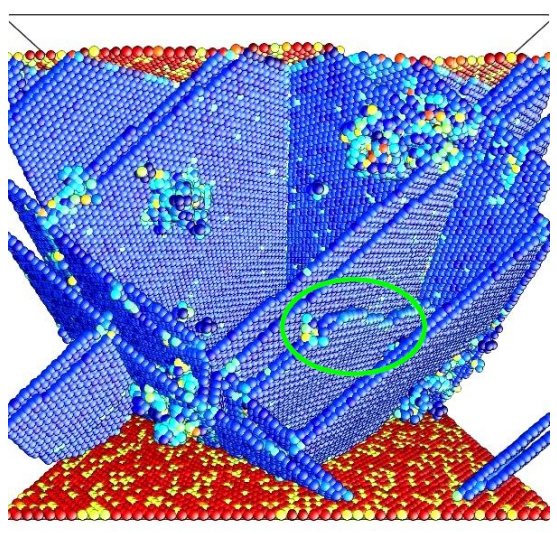

(a) $t=496.0 \mathrm{ps}$

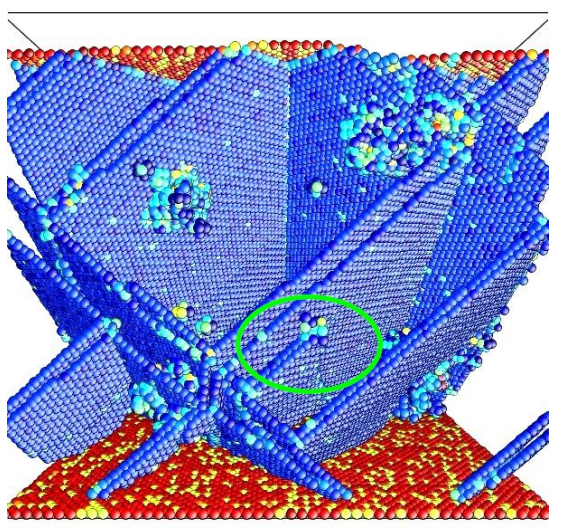

(c) $t=503.0 \mathrm{ps}$

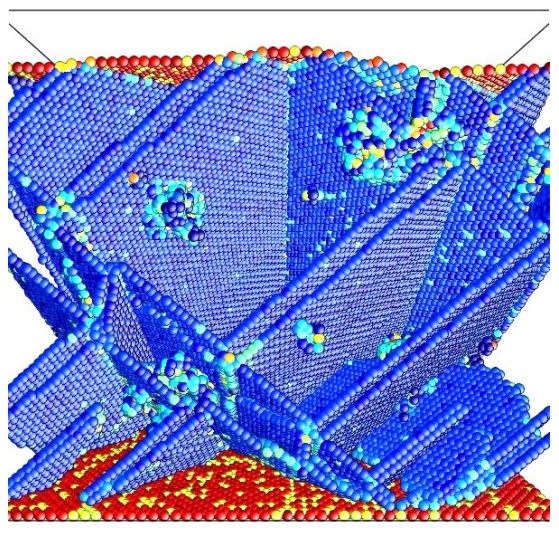

(e) $t=671.5 \mathrm{ps}$

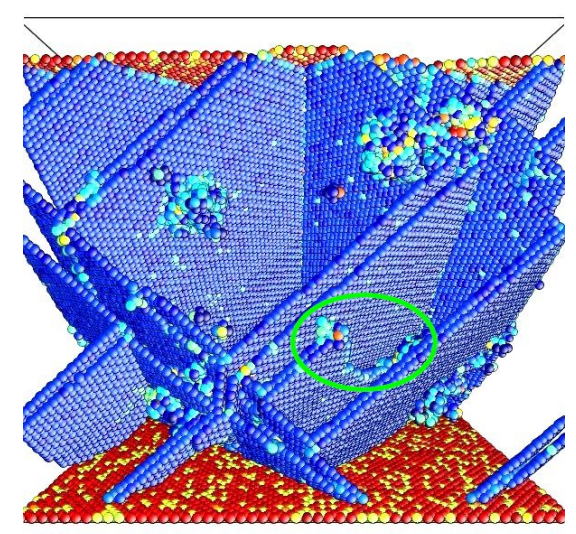

(b) $t=502.0 \mathrm{ps}$

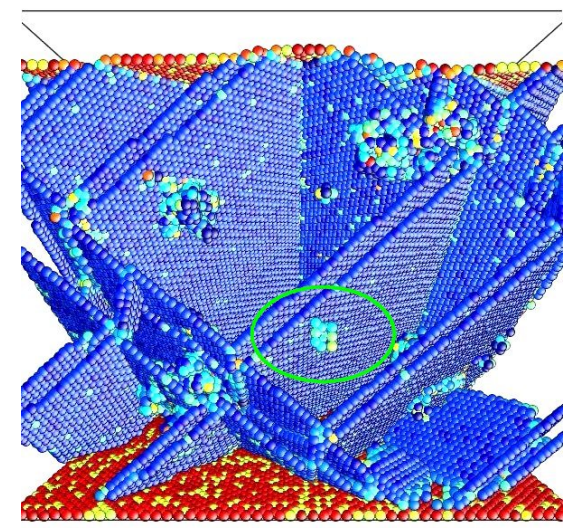

(d) $t=630.0 \mathrm{ps}$

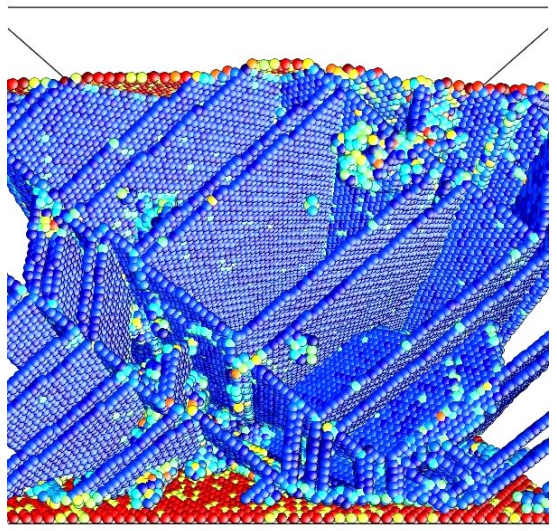

(f) $t=885.5 \mathrm{ps}$

Abbildung 5.23: Im weiteren Verlauf der Simulation bildet sich der gepinnte Zwilling wieder zurück, da er nur Stapelfehlerenergie kostet, aber nicht zur Spannungsrelaxation beiträgt. Insgesamt ist die Zahl der Zwillinge sehr viel höher als in der Zelle ohne Eisenausscheidungen. 
beginnt der gepinnte Zwilling sich zurückzubilden, da er nicht zur Spannungsrelaxation beiträgt, aber trotzdem die Grenzflächenenergie für die beiden Stapelfehler aufgewendet werden muss. Auch bei der Rückbildung dieses Zwillings ist in Abbildung 5.23 deutlich zu erkennen, wie die Eisenausscheidung den Zwilling pinnt. Dieses Pinning tritt nicht nur an der markierten Stelle, sondern vielfach in der Simulationszelle auf, so dass sich insgesamt sehr viel mehr und dafür schmalere Zwillinge im Vergleich zu der Zelle ohne Eisenausscheidungen bilden.

Außer den hier im Detail diskutierten Simulationen mit einer Dehnungsrate von $1 \cdot 10^{-7} \mathrm{fs}^{-1}$ wurden weitere Runs mit der doppelten Zellengröße und mit verschiedenen Dehnungsraten von $3 \cdot 10^{-7} \mathrm{fs}^{-1}$ und $3 \cdot 10^{-8} \mathrm{fs}^{-1}$ durchgeführt, die qualitativ identische Ergebnisse liefern. So können Artefakte wegen einer zu klein gewählten Simulationszelle (so genannte finite-size Effekte) und eine explizite Dehnungsratenabhängigkeit ausgeschlossen werden.

\subsection{Freistehende Schichten}

Solange die epitaktischen Schichten fest auf dem Substrat haften, wird sowohl ihre makroskopische Form als auch ihre Gitterstruktur massiv durch das Substrat beeinflusst. Insbesondere ein Spannungsgradient zwischen der Ober- und Unterseite einer Schicht kann zwar vom Substrat leicht aufgenommen werden, führt aber nach dem Ablösen sofort zum Aufrollen des Films. Somit lassen sich nur spannungsfreie Filme flach auf ein neues Substrat legen. Bei einem Film, während dessen Deposition aus unklarer Ursache starke Regelschwankungen der Verdampfer auftraten, ist dies in Abbildung 5.24(a) deutlich zu sehen.

Die Kristallstruktur der Filme bleibt beim Ablösen intakt, wie sich leicht an einem Vergleich der Röntgendiffraktogramme vor und nach dem Ablösen einer $\mathrm{Fe}_{70} \mathrm{Pd}_{30}$-Schicht in Abbildung 5.25 zeigen lässt. Alle dem Substrat zuzuordnenden Reflexe sind verschwunden, der einzige dem Film zuzuordnende Reflex ist unverändert.

Dass die abgelösten Filme insgesamt eine hohe Kristallqualität aufweisen, wird auch in Polfigur-Messungen deutlich, die nach dem Ablösen an einem $\mathrm{Fe}_{70} \mathrm{Pd}_{30}$-Film durchgeführt wurden. Abbildung 5.26 zeigt links eine (200)-Polfigur, in der nur ein zentraler scharfer Reflex zu erkennen ist. Weitere Reflexe wären für einen kubischen Einkristall nur unter $\Phi=90^{\circ}$ (dem Winkel zwischen den Kanten eines Würfels) zu erwarten, was aber geometrisch für ein 4-Kreis Goniometer nicht zugänglich ist. Hierbei läge die Probenoberfläche genau in der Ebene des ein- und ausfallenden Strahls, und wird daher abgeschattet. Bis zu einem Winkel von $\Phi=87^{\circ}$ sind jedoch kei- 


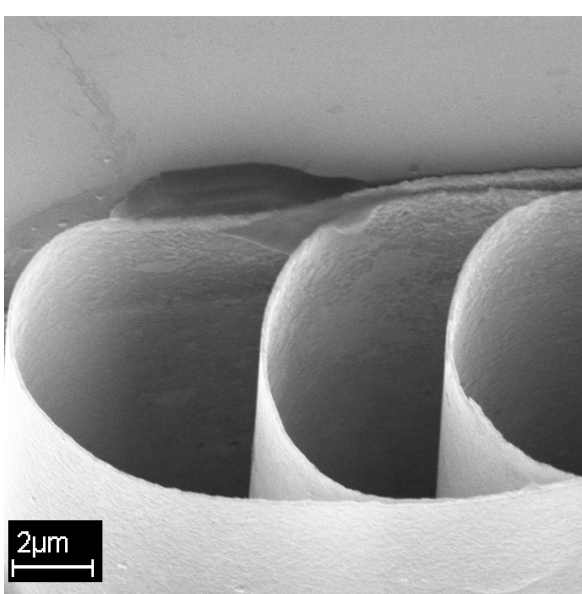

(a)

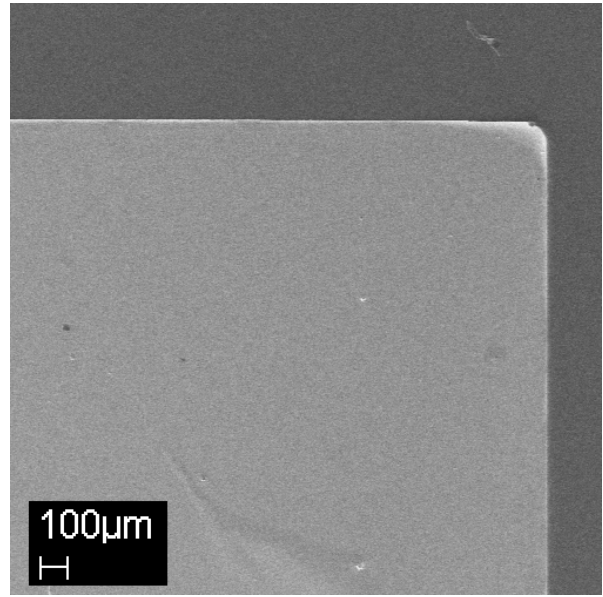

(b)

Abbildung 5.24: Spannungsinduziertes Aufrollen eines abgelösten Films, der einen Spannungsgradienten aufweist (links), im Gegensatz zu einem spannungsfreien Film, der flach aufliegt (rechts).

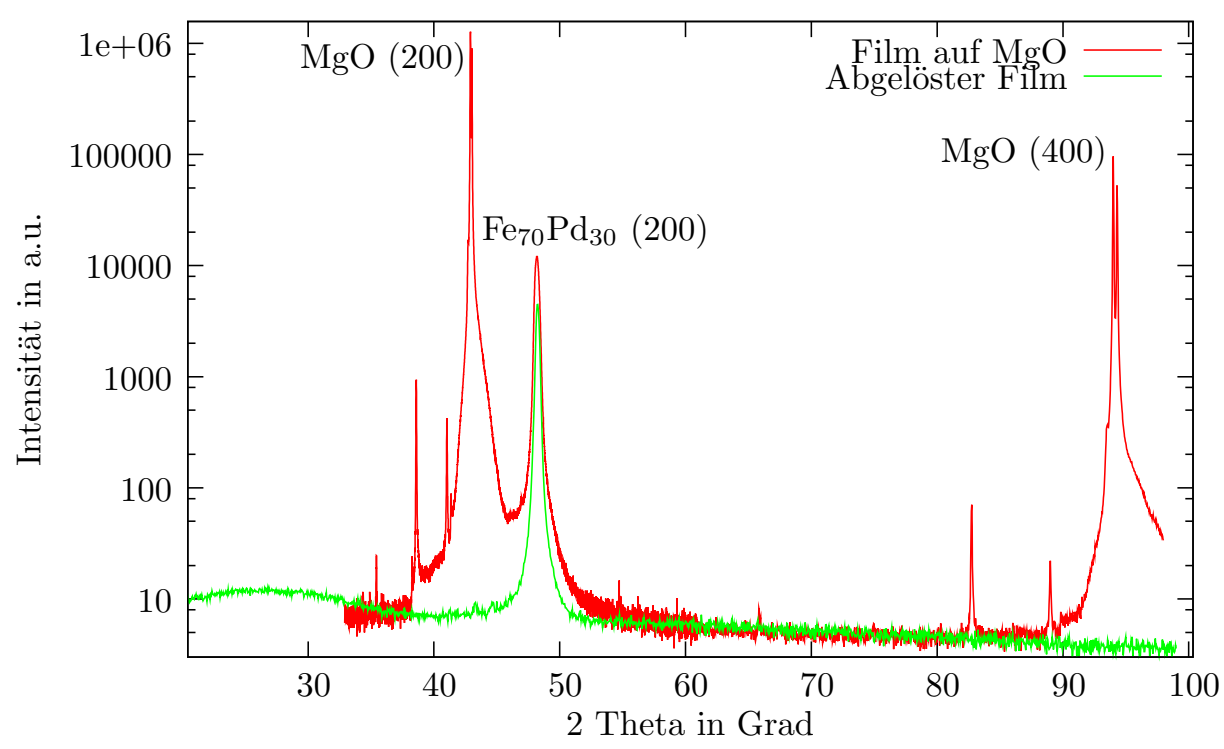

Abbildung 5.25: Röntgendiffraktogramm vor und nach dem Ablösen einer $\mathrm{Fe}_{70} \mathrm{Pd}_{30}$-Schicht vom Substrat: Alle MgO-Reflexe (die nicht indizierten Reflexe stammen von Defekten in der Kristallstruktur der Substrate) sind nach dem Ablösen verschwunden, der fcc(200)-Reflex der Schicht ist unverändert. 


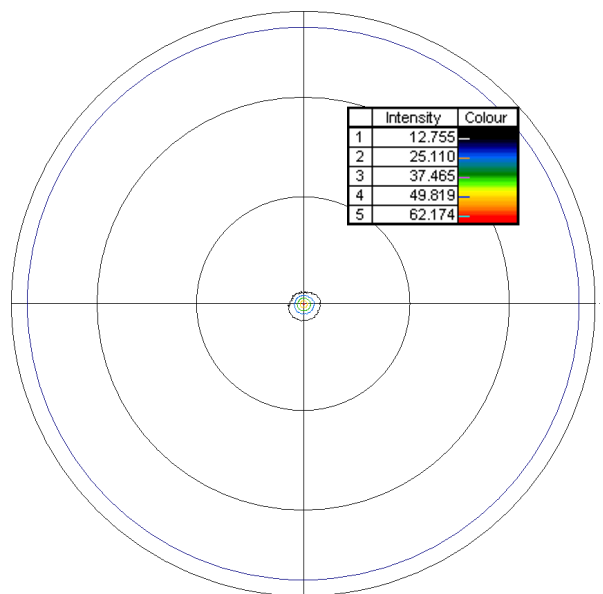

(a) (200)-Polfigur

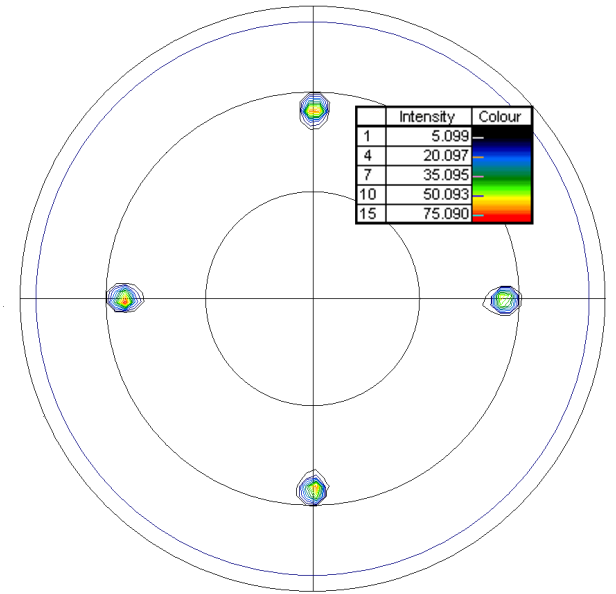

(b) (111)-Polfigur

Abbildung 5.26: Polfiguren einer einkristallinen vom Substrat abgelösten $\mathrm{Fe}_{70} \mathrm{Pd}_{30}$-Schicht .

ne weiteren Reflexe zu erkennen. Für eine (111)-Polfigur einer Schicht mit (100)-Orientierung erwartet man, genau wie in Abbildung 5.26 gezeigt, keinen zentralen Reflex, sondern vier jeweils im $\Psi=90^{\circ}$ versetzte Reflexe bei $\Phi=54.7^{\circ}$. Dies entspricht genau dem Winkel zwischen einer Raumdiagonalen und einer Kante eines Würfels. Projiziert man alle Raumdiagonalen eines Würfels auf eine der Würfelflächen, erhält man zwischen ihnen jeweils genau den Winkel von $\Psi=90^{\circ}$.

Eine hochauflösende TEM-Aufnahme einer epitaktisch auf $\mathrm{MgO}$ gewachsenen und danach mit EDTA abgelösten $\mathrm{Fe}_{70} \mathrm{Pd}_{30}$-Schicht, die zur besseren Handhabung auf ein Stück thermisch oxidiertes Silizium gelegt wurde, zeigt in Abbildung 5.27 ebenfalls deutlich, dass die Gitterstruktur des Films auch nach dem Ablösen noch intakt ist. Auch dort, wo beim Ablösen mit EDTA eine Eisenausscheidung aufgelöst wurde, und bei der FIB-Präparation auch das darunterliegende $\mathrm{SiO}_{2}$ mit abgetragen wurde, ist die Kristallstruktur nur in einem sehr schmalen Bereich von etwa $2 \mathrm{~nm}$ beeinträchtigt.

Die elastischen Eigenschaften einer freistehenden $\mathrm{Fe}_{70} \mathrm{Pd}_{30}$-Schicht wurden in Zusammenarbeit mit dem Institut für Materialphysik mittels Nanoindentation untersucht. Abbildung 5.28 zeigt den Verlauf der Auflagekraft, die notwendig ist um eine bestimmte Eindringtiefe der Berkovich-Spitze zu erreichen, und den tiefenabhängigen Elastizitätsmodul der Schicht, der hieraus durch die Berücksichtigung der genauen Spitzenform berechnet werden kann [87]. Zwischen einer Eindringtiefe von 20 und $80 \mathrm{~nm}$ erhält man so 


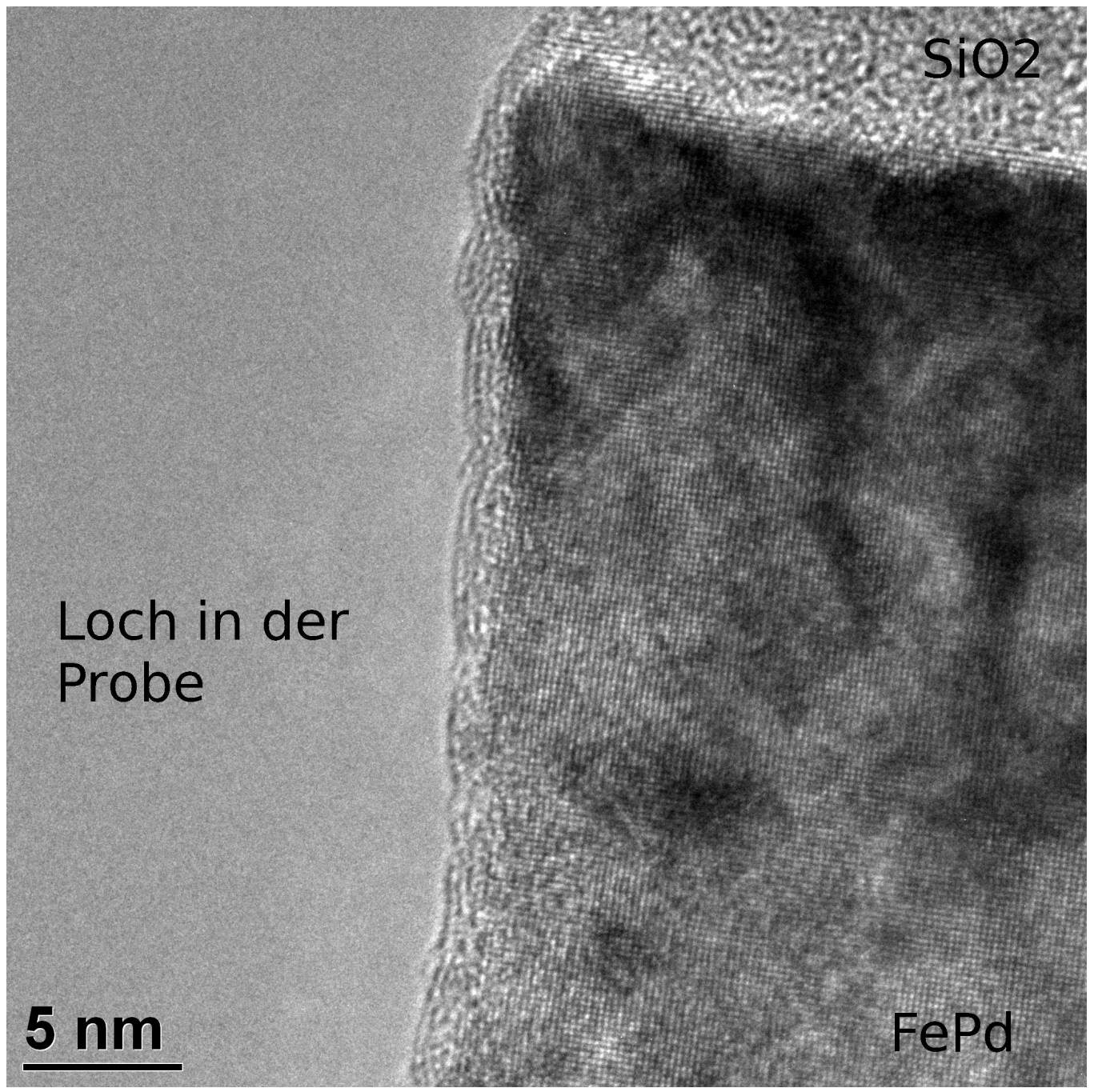

Abbildung 5.27: HRTEM-Aufnahme einer abgelösten Schicht, die auf einem $\mathrm{SiO}_{2}$-Substrat aufliegt, aus der eine Eisenausscheidung herausgelöst ist. 
einen mittleren E-Modul von 13,9 GPa. In diesem Bereich sind auch mehrere so genannte Pop-ins zu erkennen. Dabei handelt es sich um Ereignisse, bei denen ohne nennenswerte Kraftänderung die Spitze ruckartig tiefer in die Schicht eindringt. Der erste Pop-in tritt bei einer Auflagekraft von $0.034 \mathrm{mN}$ auf, die beiden anderen etwa bei der doppelten und der dreifachen Auflagekraft, nämlich bei $0.076 \mathrm{mN}$ und $0.11 \mathrm{mN}$. Ab einer Tiefe von $100 \mathrm{~nm}$ steigt der E-Modul steil an, da hier der Einfluss des Substrats (dargestellt in Abbildung 5.29) deutlich zunimmt.

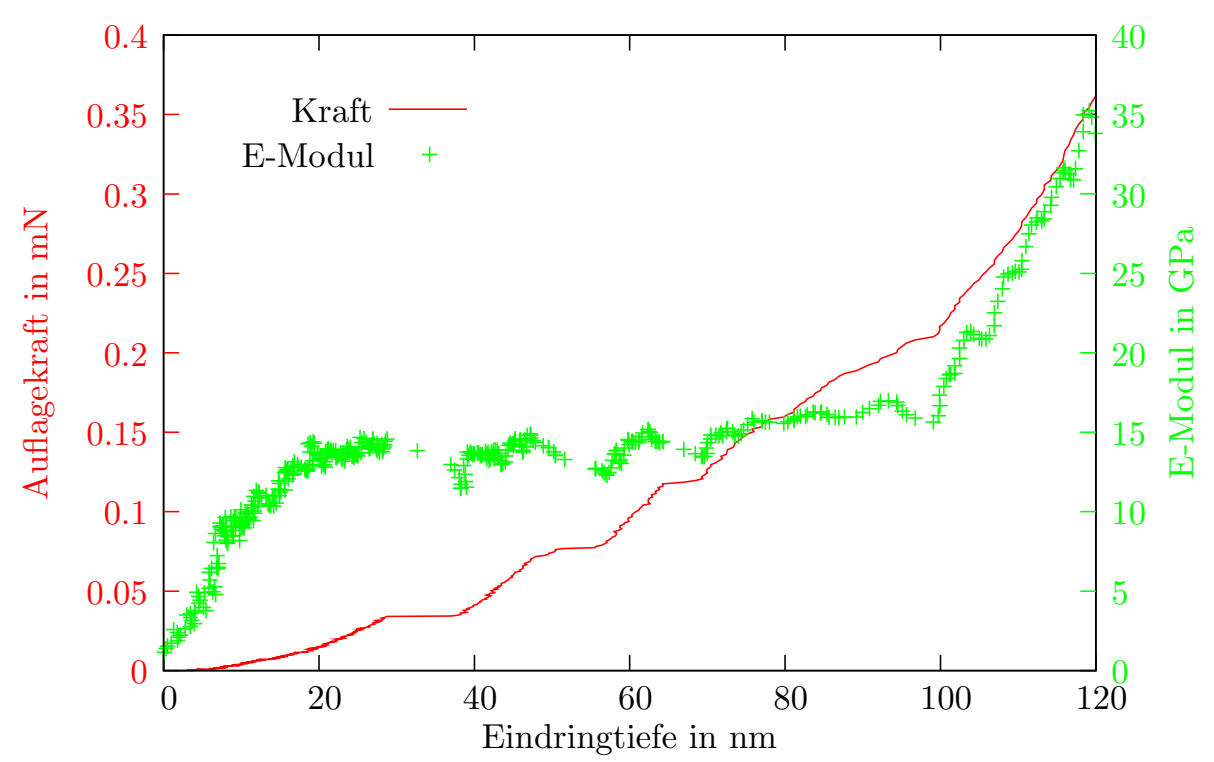

Abbildung 5.28: Nanoindentation mit einer Berkovich-Spizte an einer abgelösten $\mathrm{Fe}_{70} \mathrm{Pd}_{30}$-Probe auf einem $\mathrm{SiO}_{2}$-Substrat.

Werden freistehende Schichten nach dem Ablösen getempert, so kann man auch hier den Einfluss des MgO-Substrats auf die Kristallstruktur sehr deutlich feststellen. Bei einer Zusammensetzung von $72 \%$ Fe und $28 \%$ Pd kann man z. B. beobachten, dass sich je nach Temperatur und Dauer der Temperaturbehandlung verschieden Strukturen stabilisieren lassen. Hierfür wurde ein Film zunächst in mehrere Teile zerteilt, die gemeinsam in einem Gefäß mit Natriumhydrogencarbonatlösung abgelöst wurden, so dass diese einzelnen Teile identische Ausgangsbedingungen haben. Anschließend wurden die einzelnen Teilstücke in Quarzampullen eingeschmolzen, und bei $900{ }^{\circ} \mathrm{C}$ oder $1000^{\circ} \mathrm{C}$ kurz (15 Minuten) oder lang (20 Stunden) getempert. Als zusätzlicher Vergleich wurde auch ein Probenteil getempert, von dem das $\mathrm{MgO}$ noch nicht vollständig abgelöst war. Im Ausgangszustand weist die Probe 


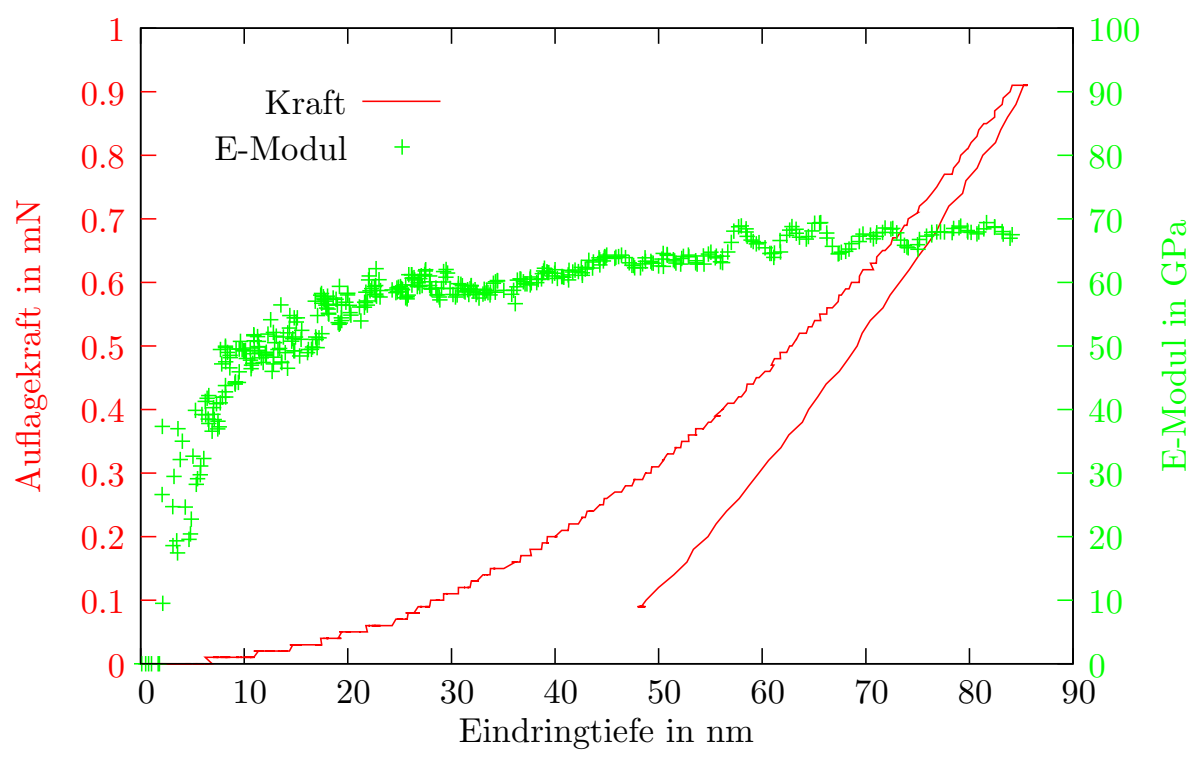

Abbildung 5.29: Nanoindentation mit einer Berkovich-Spizte an einem reinen $\mathrm{SiO}_{2}$-Substrat.

die fcc-Struktur auf. Außer dem (200)fcc-Peak bei 48, $2^{\circ}$ ist nur der (110)Peak von $\alpha$-Eisen zu sehen. Bei allen getemperten Proben in Abbildung 5.30 ist dieser Eisenpeak verschwunden, so dass davon auszugehen ist, dass diese Eisenausscheidungen nicht schon während der Deposition sondern erst beim Abkühlen nach der Deposition entstanden sind. Nach dem Tempern wird die Probe so rasch abgeschreckt, dass ein Entmischen verhindert wird.

Die Struktur der Probe, an der das $\mathrm{MgO}$ noch nicht vollständig abgelöst ist, ist auch nach 20 Stunden bei $900^{\circ} \mathrm{C}$ bis auf das Auflösen der Eisenausscheidungen unverändert. Hier ist außer dem fcc(200)-Peak der Schicht bei $48,2^{\circ}$ nur der MgO-Peak zu sehen. Schon die für 15 Minuten bei $900^{\circ} \mathrm{C}$ getemperte Probe ohne Substratrest weicht dagegen deutlich von der auf dem Substrat getemperten Schicht ab: Der (200)Peak hat sich deutlich zu größeren Winkeln verschoben, das Hauptmaximum liegt nun bei $48,52^{\circ}$ und ist

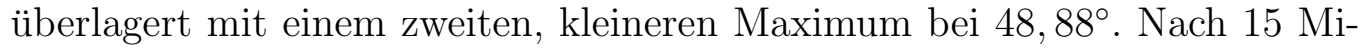
nuten tempern bei $1000^{\circ} \mathrm{C}$ ist von dem Maximum bei $48.52^{\circ}$ nur noch ein schwacher Rest zu sehen, wogegen ein deutlicher fct-Peak bei $49.2^{\circ}$ auftritt, und einem weiteren Peak bei $43.72^{\circ}$, der der bcc-Struktur zugeordnet werden kann, und der auch bei langem Tempern bei $900{ }^{\circ} \mathrm{C}$ nicht auftritt. Diese Tendenz setzt sich weiter fort wenn die Probe für lange Zeit bei $1000^{\circ} \mathrm{C}$ getempert wird: Nach 20 Stunden ist nur noch der bcc-Peak bei $43.33^{\circ} \mathrm{zu}$ sehen, der fcc(200)-Peak ist vollständig verschwunden. 


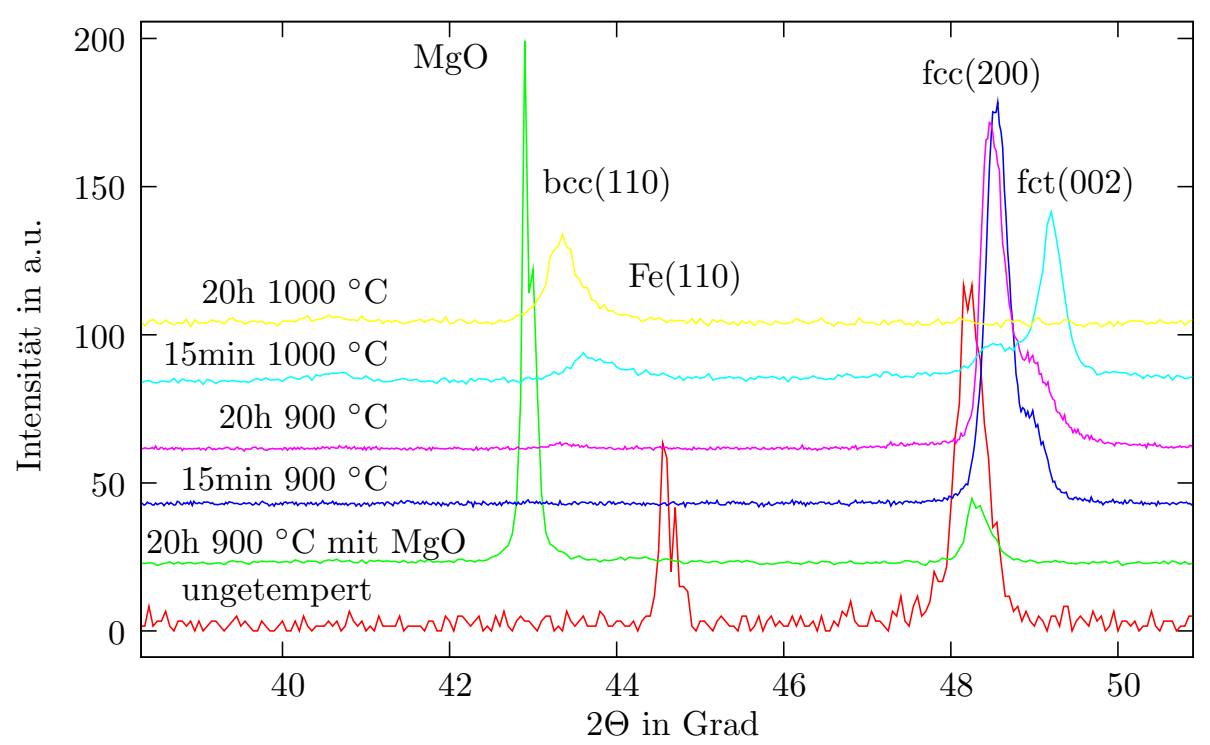

Abbildung 5.30: $\mathrm{Fe}_{72} \mathrm{Pd}_{28}$-Proben: nach dem Tempern bei $900{ }^{\circ} \mathrm{C}$ erhält man die fcc/fct-Struktur, bei $1000{ }^{\circ} \mathrm{C}$ die bcc-Struktur.

\subsection{Martensitische Umwandlungen}

Dieses Kapitel beschäftigt sich mit den Bedingungen, unter denen ein Martensitübergang abläuft. Um die Einflüsse der Mikrostruktur und des Substrats zu untersuchen, werden hierfür zunächst polykristalline Schichten, die bei Raumtemperatur aufgedampft und nachträglich getempert wurden untersucht, aber danach auch epitaktisch gewachsene Filme vor und nach dem Ablösen vom Substrat.

\subsubsection{Polykristalline Filme auf $\mathrm{SiO}_{2}$}

Messungen des elektrischen Widerstands erlauben es, mit geringem Experimentellem Aufwand einen ersten Hinweis auf einen Martensitübergang zu erhalten [108, 109, 32]. In Kooperation mit Sven Hamann wurden in der Arbeitsgruppe von A. Ludwig an der Universität Bochum an verschiedenen polykristallinen Proben, die bei Raumtemperatur auf $\mathrm{SiO}_{2}$ aufgedampft und dann 10 Minuten bei $900^{\circ}$ getempert wurden, temperaturabhängige Widerstandsmessungen durchgeführt. Jede Änderung der Gitterstruktur ändert die Streuwahrscheinlichkeit der Elektronen, und sollte daher im Widerstand zu erkennen sein. In Abbildung 5.31 sind die gemessenen Widerstandswerte für eine $\mathrm{Fe}_{70} \mathrm{Pd}_{30}$-Probe und eine $\mathrm{Fe}_{72} \mathrm{Pd}_{28}$-Probe gezeigt. Während die $\mathrm{Fe}_{70} \mathrm{Pd}_{30^{-}}$ 


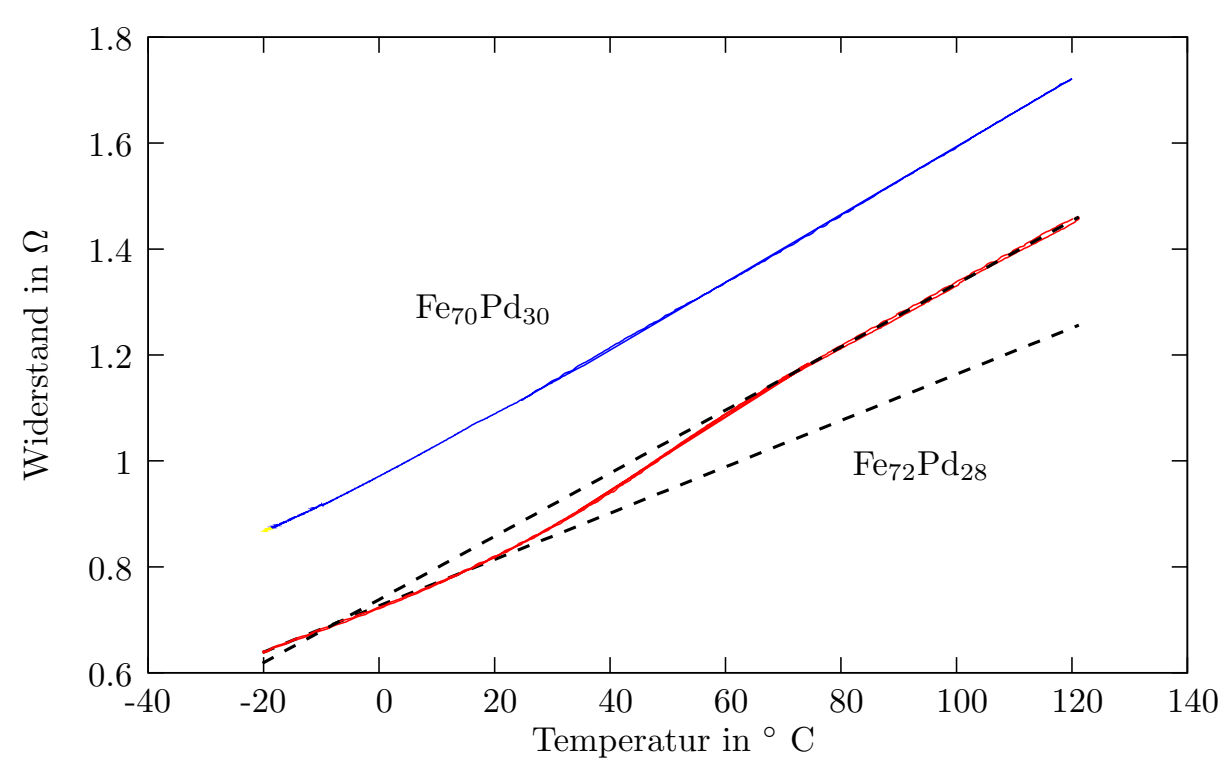

\begin{abstract}
Abbildung 5.31: Widerstand in Abhängigkeit der Temperatur einer polykristallinen $\mathrm{Fe}_{70} \mathrm{Pd}_{30}$-Schicht, die keine Transformation zeigt, und einer transformierenden $\mathrm{Fe}_{72} \mathrm{Pd}_{28}$-Schicht. In rot und blau die gemessenen Werte, in schwarz an den Bereich über $60^{\circ} \mathrm{C}$ und an den Bereich unter $20^{\circ} \mathrm{C}$ angepasste Geraden.
\end{abstract}

Probe über den gesamten Temperaturbereich ein lineares Verhalten zeigt, sind an der $\mathrm{Fe}_{72} \mathrm{Pd}_{28}$-Probe zwei Auffälligkeiten zu erkennen: Bei einer Temperatur von $60^{\circ} \mathrm{C}$ und bei $20^{\circ} \mathrm{C}$ ändert sich die Steigung deutlich. Oberund unterhalb dieses Bereichs steigt der Widerstand linear mit der Temperatur an, und weist eine recht ähnliche Steigung auf. Für jede Probe wurde der zugängliche Temperaturbereich mehrfach durchgefahren und dabei jeweils Daten aufgenommen. Hierbei werden die Ergebnisse der vorhergegangenen Messungen sehr gut reproduziert, auch in der Probe, deren Steigung sich verändert, ist keine Hysterese zu erkennen.

Um diesen indirekten Hinweis auf einen Martensitübergang in der $\mathrm{Fe}_{72} \mathrm{Pd}_{28^{-}}$ Probe zu untermauern, wurden an den Proben außerdem Röntgenmessungen bei verschiedenen Temperaturen durchgeführt, die in Abbildung 5.32 dargestellt sind. Deutlich zu erkennen ist, dass der fcc(111)-Peak bei allen dargestellten Temperaturen unverändert bleibt, während der fcc(200)-Peak unter $60^{\circ} \mathrm{C}$ nicht zu erkennen ist, und bei höheren Temperaturen immer deutlicher ausgeprägt ist. Die Probe zeigt also einen Martensitübergang. Bei Raumtemperatur befindet sich die Probe in einer martensitischen Struktur und geht bei etwa $60^{\circ} \mathrm{C}$ in den Austenit über. Die nicht transformierende $\mathrm{Fe}_{70} \mathrm{Pd}_{30^{-}}$ 


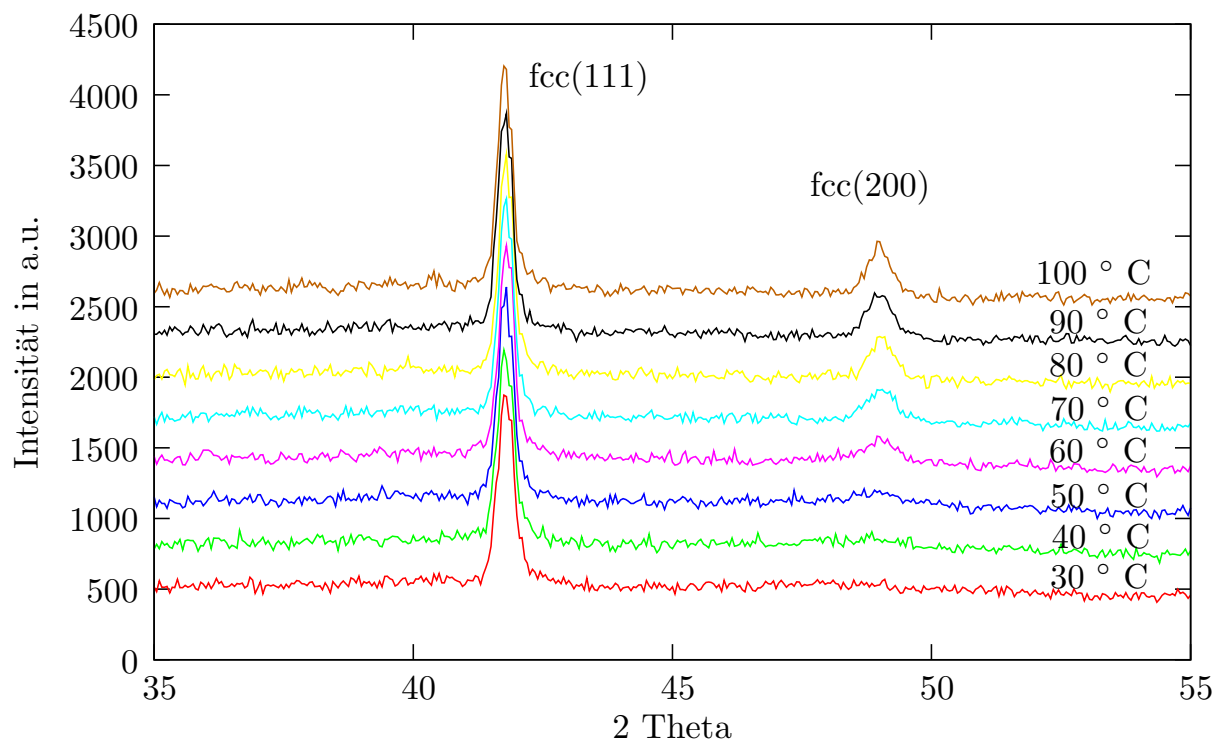

Abbildung 5.32: Röntgenbeugung an einer polykristallinen $\mathrm{Fe}_{72} \mathrm{Pd}_{28}$-Schicht bei verschiedenen Temperaturen: Während der fcc(111)-Peak unverändert bleibt, ist der (200)-Peak nur oberhalb von $60^{\circ} \mathrm{C}$ zu erkennen.

Probe liegt dagegen bei Raumtemperatur als Austenit vor.

In der Gruppe von Prof. Gatzen am IMT Hannover wurden sowohl an der nicht transformierenden austenitischen $\mathrm{Fe}_{70} \mathrm{Pd}_{30}$-Schicht, als auch an der bei Raumtemperatur martensitischen $\mathrm{Fe}_{72} \mathrm{Pd}_{28}$-Schicht mittels eines Nanoindenters die elastischen Eigenschaften vermessen. Die Steigungen der KraftEindringtiefe-Kurve (Abbildungen 5.33 und 5.34) während des Belastens unterscheiden sich zwischen den verschiedenen Messungen auf einer Probe sehr stark. Dies ist auf die ungleichmäßige, körnige Probenoberfläche (siehe Abbildung 5.35) zurückzuführen, und beeinflusst, je nach dem ob die Spitze auf einen flachen Bereich oder die Flanke eines Korns drückt, vor allem den Grad der plastischen Verformung. Betrachtet dagegen die Steigung der Kurve beim Entlasten, die den elastischen Teil der Verformung wiedergibt, so sind diese für alle Kurven vergleichbar und liefern sowohl für die austenitische als für die martensitische Probe einen E-Modul von etwa 120 GPa.

Betrachtet man das Oberflächenrelief der Probe in der in Abbildung 5.35 dargestellten AFM-Aufnahme, erkennt man eine körnige Struktur mit Korngrößen im Bereich eines halben Mikrometers. Dies legt nahe, dass eine der Ursachen der großen Streuung der Nanoindenter-Messungen in der Probentopologie zu suchen ist. Darüber hinaus sind diese Messungen mit weiteren messtechnischen Problemen behaftet, die in Kapitel 6.4 diskutiert werden. 


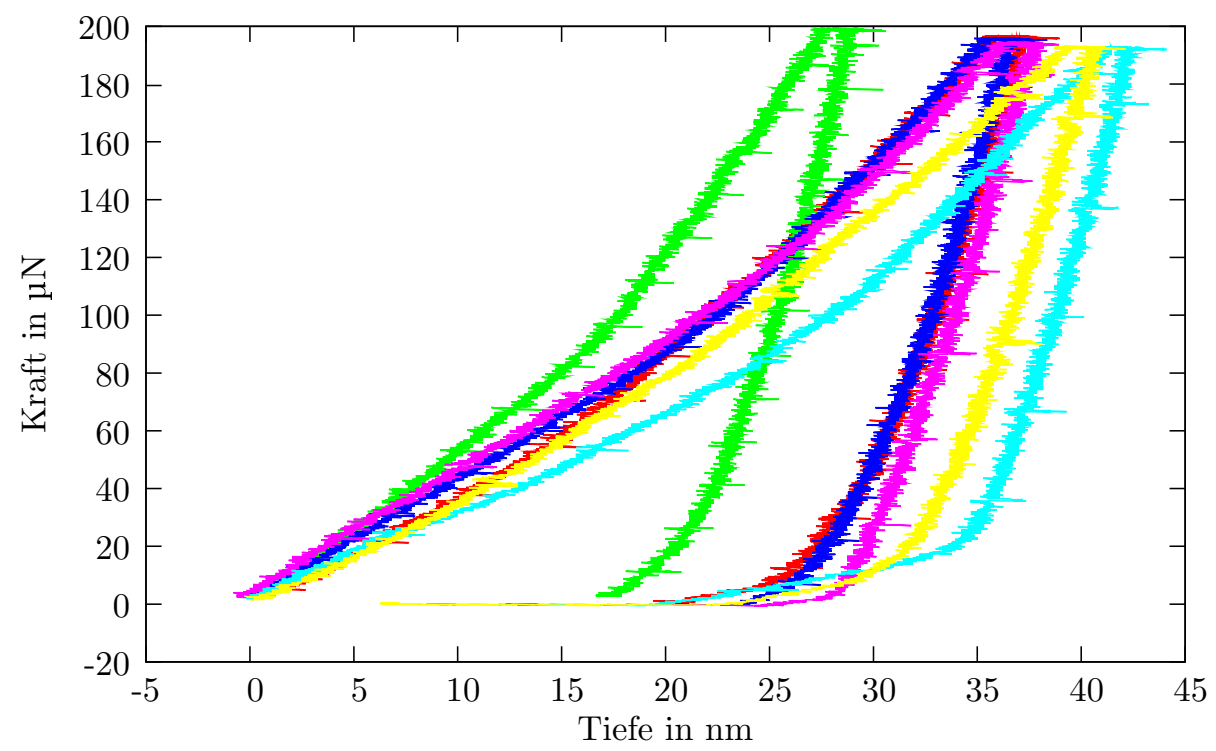

Abbildung 5.33: Kraft-Eindringtiefe-Kurven der austenitischen Probe

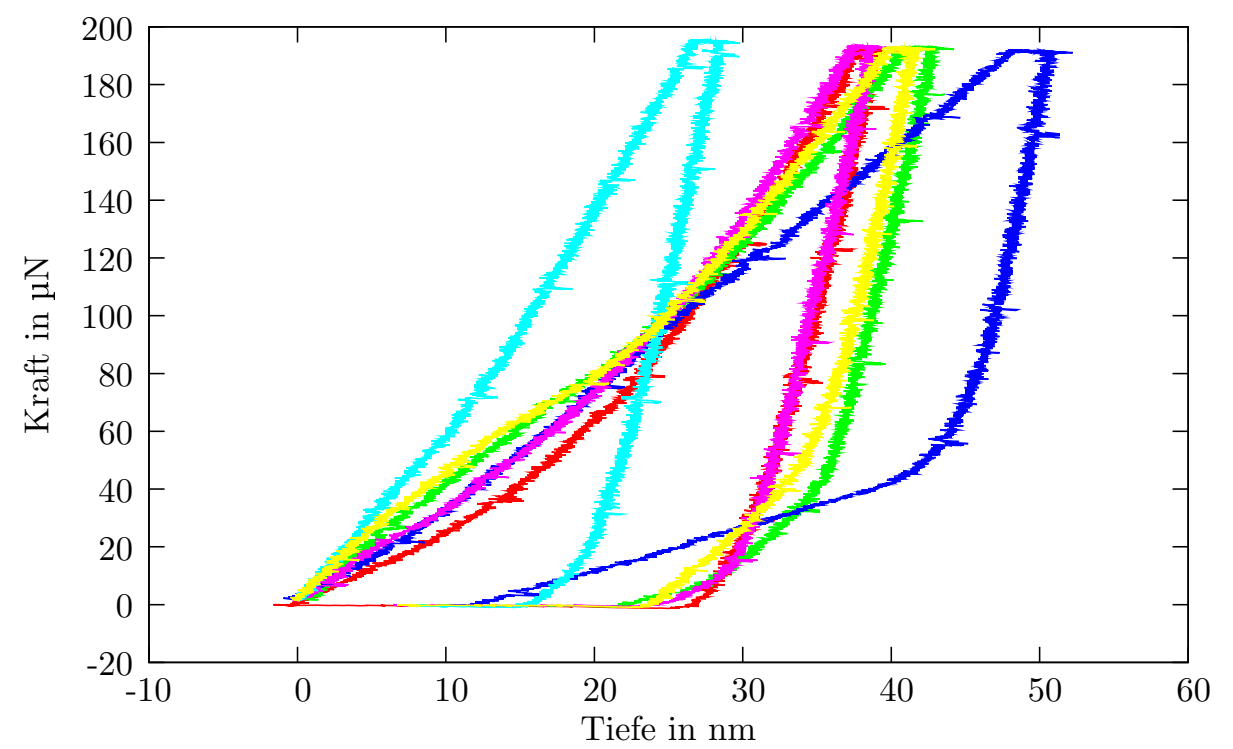

Abbildung 5.34: Kraft-Eindringtiefe-Kurven der martensitischen Probe 


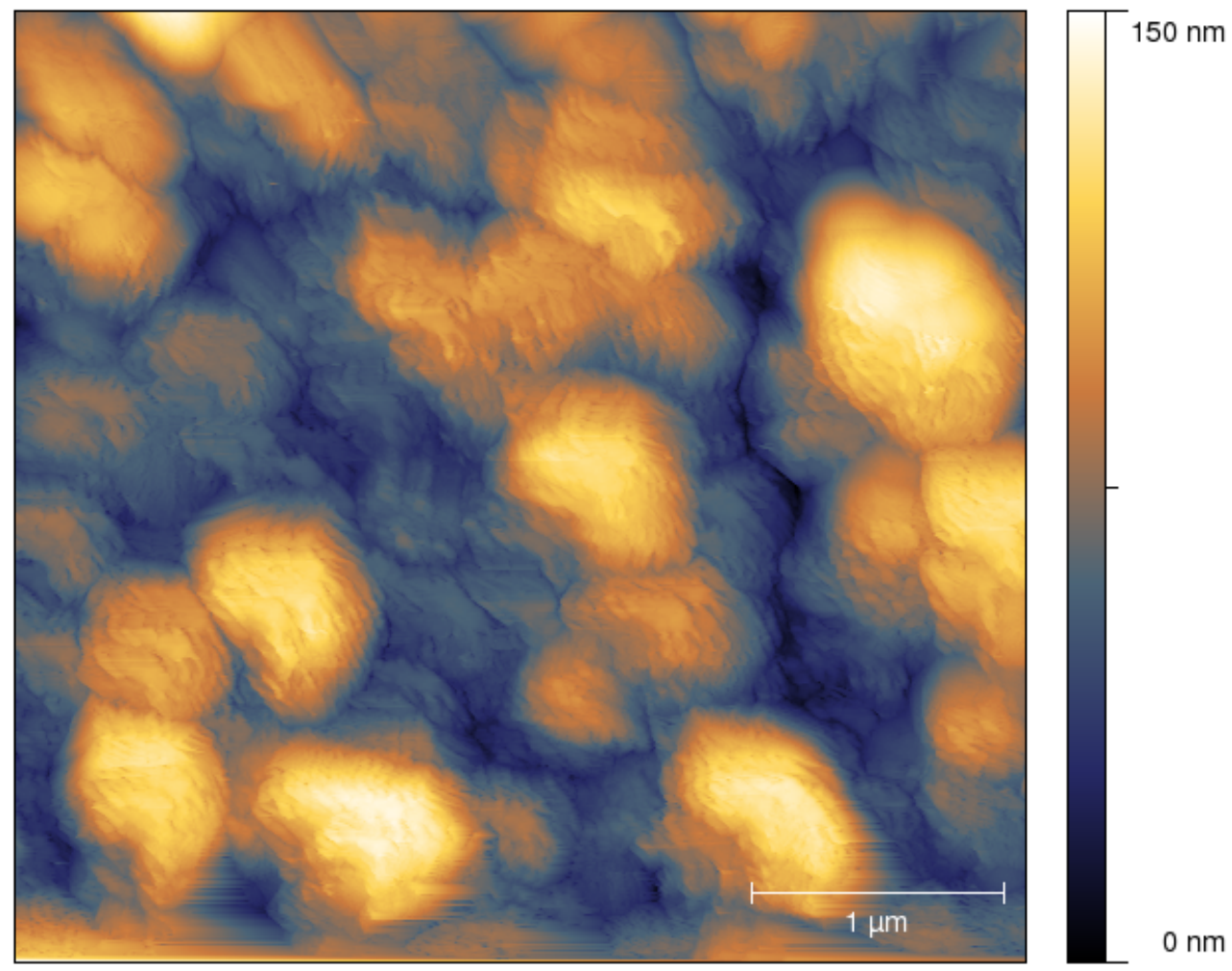

Abbildung 5.35: AFM-Topographie der polykristallinen martensitischen $\mathrm{Fe}_{72} \mathrm{Pd}_{28}$-Schicht 


\subsubsection{Freistehende einkristalline Filme}

Im Vergleich zwischen epitaktischen Schichten, die fest auf dem MgO-Substrat aufliegen, und vom $\mathrm{MgO}$ abgelösten Filmen lässt sich der Einfluss des Substrats auf mögliche Änderungen der Gitterstruktur untersuchen. Ein sehr deutliches Beispiel stellt die Untersuchung des Invar-Effekts dar, bei dem das Substrat das Verhalten maßgeblich bestimmt. Eine nicht vom MgO-Substrat abgelöste $\mathrm{Fe}_{70} \mathrm{Pd}_{30}$-Schicht zeigt in Abbildung 5.36 oberhalb von $0^{\circ} \mathrm{C}$ eine leicht reduzierte thermische Ausdehnung. Berechnet man den thermischen Ausdehnungskoeffizienten des Films auf dem Substrat in diesem Bereich, erhält man einen Wert von $12 \cdot 10^{-6} \mathrm{~K}^{-1}$, der sehr nahe am thermischen Ausdehnungskoeffizienten von $\mathrm{MgO}$ mit etwa $11 \cdot 10^{-6} \mathrm{~K}^{-1}$ liegt [110] liegt. Im Gegensatz dazu ist in einer abgelösten Schicht ein sehr ausgeprägter InvarEffekt zu erkennen: Die Gitterkonstante hängt hier in einem Temperaturbereich oberhalb von $0{ }^{\circ} \mathrm{C}$ kaum von der Probentemperatur ab, die thermische Ausdehnung der Probe ist in diesem Bereich sehr viel geringer als bei tieferen Temperaturen. Es ist daher davon auszugehen, dass die Dehnung der Schicht auf dem MgO-Substrat im Wesentlichen von der thermische Ausdehnung des Substrats verursacht wird, und dass die sehr weiche Schicht (siehe Abbildung 5.28) mitgedehnt wird.

Bei epitaktischen $\mathrm{Fe}_{70} \mathrm{Pd}_{30}$-Filmen auf $\mathrm{MgO}$, und solchen, die vom Substrat abgelöst wurden, konnte bis zu Temperaturen von $-140^{\circ} \mathrm{C}$ kein Martensitübergang nachgewiesen werden. Werden diese freistehenden Filme jedoch nach dem Ablösen für 30 Minuten bei $900{ }^{\circ} \mathrm{C}$ getempert, zeigt sich in Abbildung 5.37 bei tiefen Temperaturen eine deutliche Änderung der Gitterstruktur. Unterhalb von $-45^{\circ} \mathrm{C}$ tritt nun ein weiterer Peak bei $43,4^{\circ}$ auf, der sich als bct- bzw. bcc(110)-Peak identifizieren lässt. Mit dem Auftreten der raumzentrierten Struktur nimmt gleichzeitig der fcc(200)-Peak deutlich an Intensität ab, und weist eine Schulter bei kleineren Winkeln auf, die als fct(200)-Peak gedeutet werden kann. Dieser hebt sich deutlich von der temperaturunabhängigen Stufe vor dem fcc(200)-Reflex ab, die dadurch verursacht wird, dass der verwendete Nickel-Filter die Röntgenstrahlung nicht vollständig monochromatisiert. Besonders bemerkenswert ist, dass der bctPeak beim Aufheizen wieder verschwindet, und bei erneutem Abkühlen wieder auftritt. Diese Umwandlung ist also reversibel.

Röntgenmessungen in einem 2-Kreis-Goniometer zeigen nur die Ebenenabstände senkrecht zur Schichtebene. Um den Mechanismus dieses reversiblen Übergangs von der flächenzentrierten zu einer raumzentrierten Struktur weiter aufzuklären, wurden darüberhinaus weitere Röntgenstreuexperimente in Transmission durchgeführt. Hierfür wurde die freistehende Schicht so in einen Wachsblock eingebettet, dass sie senkrecht zur Filmoberfläche von der Rönt- 


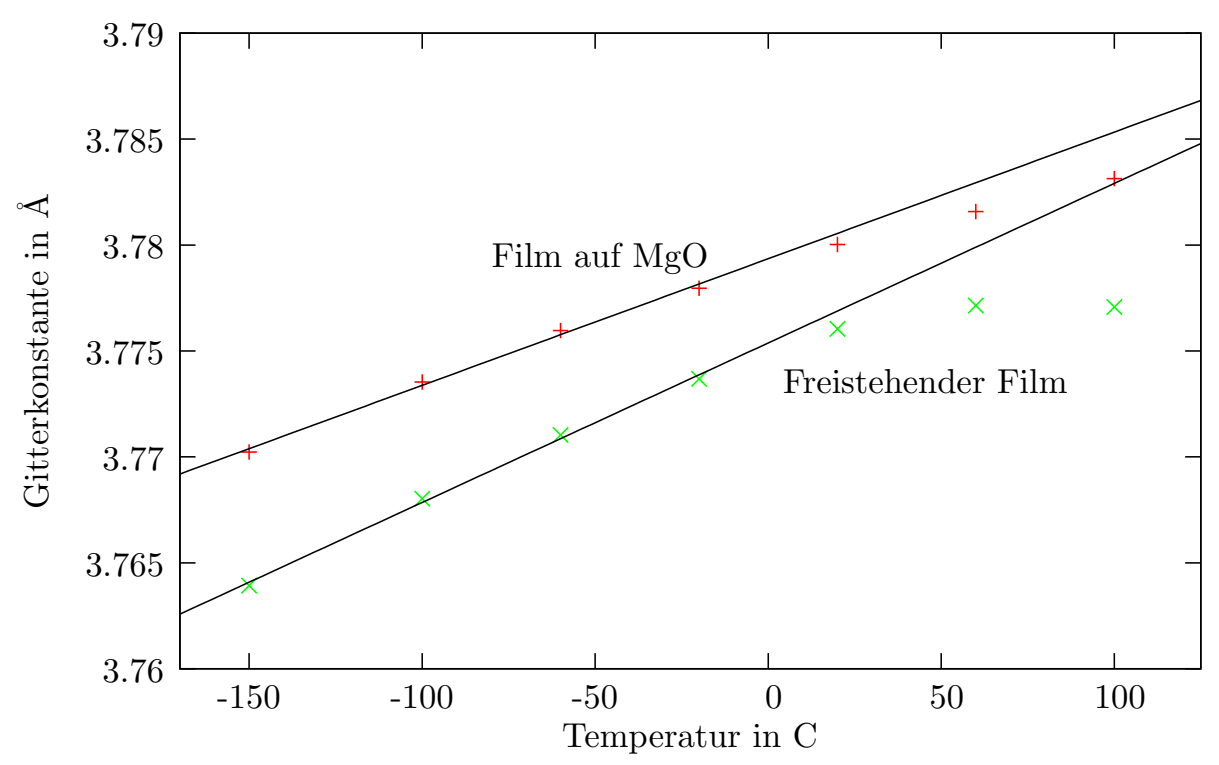

Abbildung 5.36: Während eine vom Substrat abgelöste $\mathrm{Fe}_{70} \mathrm{Pd}_{30}$-Schicht oberhalb von $0^{\circ} \mathrm{C}$ fast keine thermische Ausdehnung mehr zeigt, ist diese bei einer Schicht auf $\mathrm{MgO}$ nur etwas verringert. Aus [111].

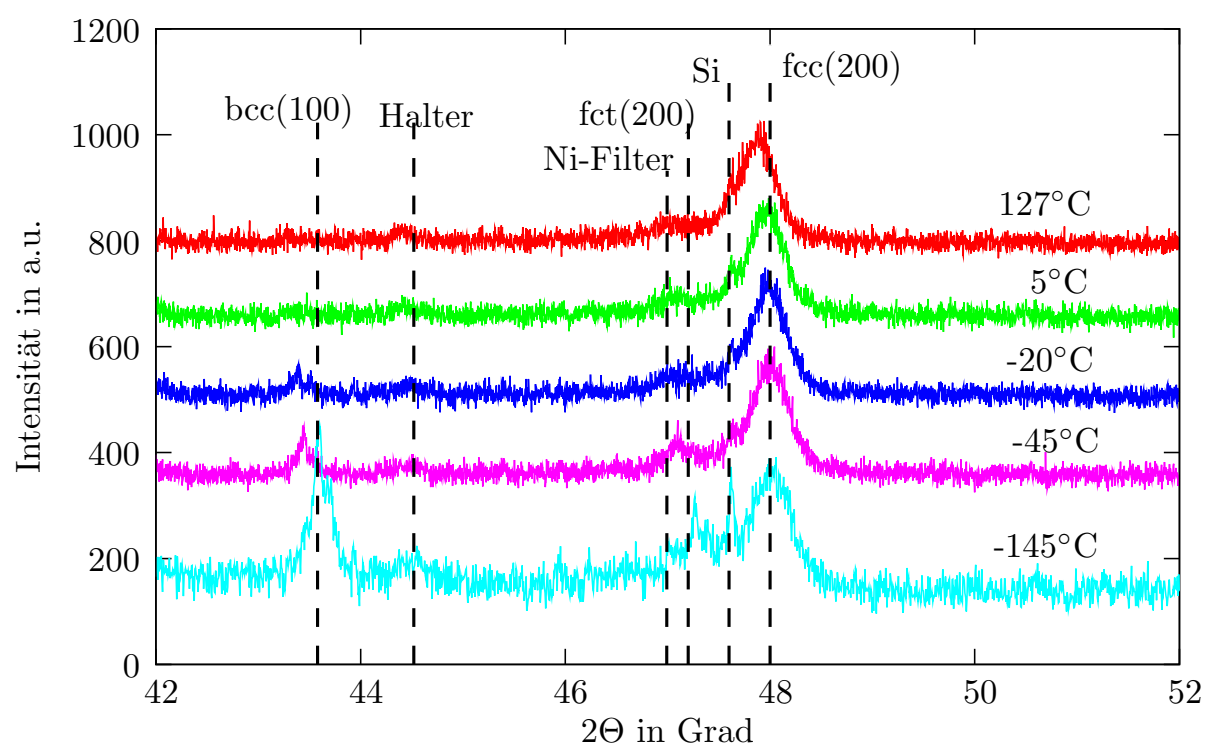

Abbildung 5.37: Röntgenbeugung an einer freistehenden getemperten $\mathrm{Fe}_{70} \mathrm{Pd}_{30}$-Probe. Bei tiefen Temperaturen treten zusätzlich der bct(110) und ein schwacher fct(200)-Peak auf. Aus [111]. 
genstrahlung durchdrungen wird und die Beugungsfigur hinter der Probe mit einem Flächendetektor aufgezeichnet werden konnte. Das bei Raumtemperatur aufgenommene Beugungsbild (Abbildung 5.38(a)) zeigt zwei deutliche

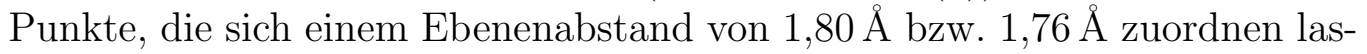
sen und die um 90 Grad gegeneinander versetzt sind. Hierbei handelt es sich um die $\{200\}$-Reflexe der fcc-Struktur. Außer diesen punktförmigen Reflexen sind weitere kreisförmige Beugungsringe zu erkennen, die vom Probenhalter und dem Wachs stammen, in dem die Probe eingebettet wurde. Dass außer dem (020)- und dem (002)-Reflex nicht auch symmetrisch dazu die (02 0 )und $(00 \overline{2})$-Reflexe zu sehen sind, ist dadurch zu erklären, dass die Probe aufgrund der Einbettung in das Wachs nicht exakt zum Strahl ausgerichtet werden kann.

Kühlt man die Probe nun auf eine Temperatur von $-100{ }^{\circ} \mathrm{C}$ ab, treten in Abbildung 5.38(b) weitere Reflexe in Erscheinung: Es werden die mit einem der Beugungsringe des Wachs überlagerten Reflexe mit einem Ebenenabstand von 1,96 $\AA$ bzw. 2,03 $\AA$ deutlich erkennbar, die genau die selbe Orientierung wie die fcc $\{200\}$-Reflexe aufweisen. Diese Reflexe können als $\{110\}$-Reflexe der bct- oder bcc-Struktur identifiziert werden, und ergeben für diese einen Gitterparameter von 2,87 $\AA$ bzw. 2,77 $\AA$. Diese Werte weisen einen ähnlichen Unterschied wie die fcc $\{200\}$-Reflexe auf, so dass nicht eindeutig zwischen einer kubischen und einer tetragonalen Struktur unterschieden werden kann. Die tetragonale Verzerrung lässt sich aber zu $c / a<1,03$ abschätzen. Daher wird die Struktur auch im folgenden als bct-Struktur bezeichnet.

Darüber hinaus ist ein weiterer Reflex sichtbar, der zu einem Ebenenabstand von 2,40 ̊ gehört und der ca. 10 Grad gegen die (100)-Richtung des fcc-Gitters verdreht ist. Dieser Reflex lässt sich weder der fcc- noch der bcc-Struktur zuordnen.

Betrachtet man die temperaturabhängige Magnetisierung dieses Films (Abbildung 5.39), findet sich dort ebenfalls eine Signatur des Martensitübergangs. Vergleicht man hier den Verlauf der auf den Wert bei $125^{\circ} \mathrm{C}$ normierten Magnetisierung der Probe vor und nach dem Tempern, erkennt man, dass die getemperte Probe zunächst beim ersten Abkühlen parallel zum Verlauf vor dem Tempern ansteigt. Von diesem Verlauf weicht die Kurve ab einer Temperatur von etwa $-45^{\circ} \mathrm{C}$ ab, und ist über einen Bereich von über $150 \mathrm{Grad}$ konstant. Beim Erwärmen folgt die Kurve zunächst in etwa dem Verlauf des Abkühlens. Sobald die Probe allerdings in den Bereich von Raumtemperatur und darüber hinaus erwärmt wird, zeigt sich ein anderer Verlauf. Dieser ist darauf zurückzuführen, dass die Probe nach dem Tempern und Abschrecken zum ersten mal wieder erwärmt wird. Dadurch können beim Abschrecken eingefrorene Defekte zumindest teilweise relaxieren. Da nun die Defektkonzentration abnimmt, ist die Magnetisierung weniger gepinnt, und man erhält 


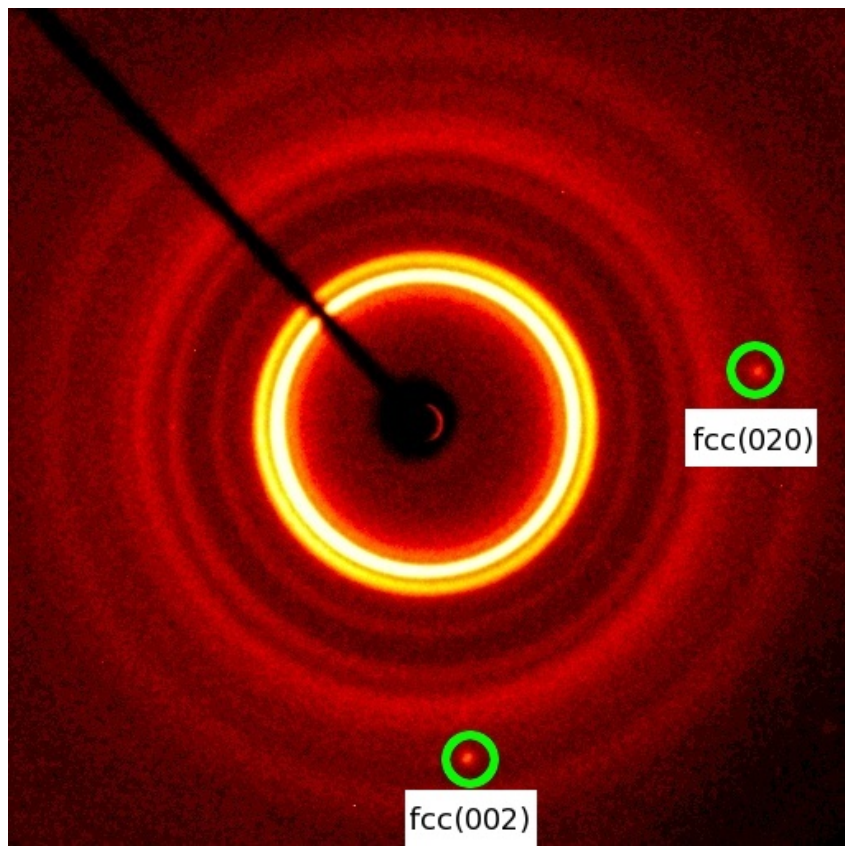

(a) Raumtemperatur

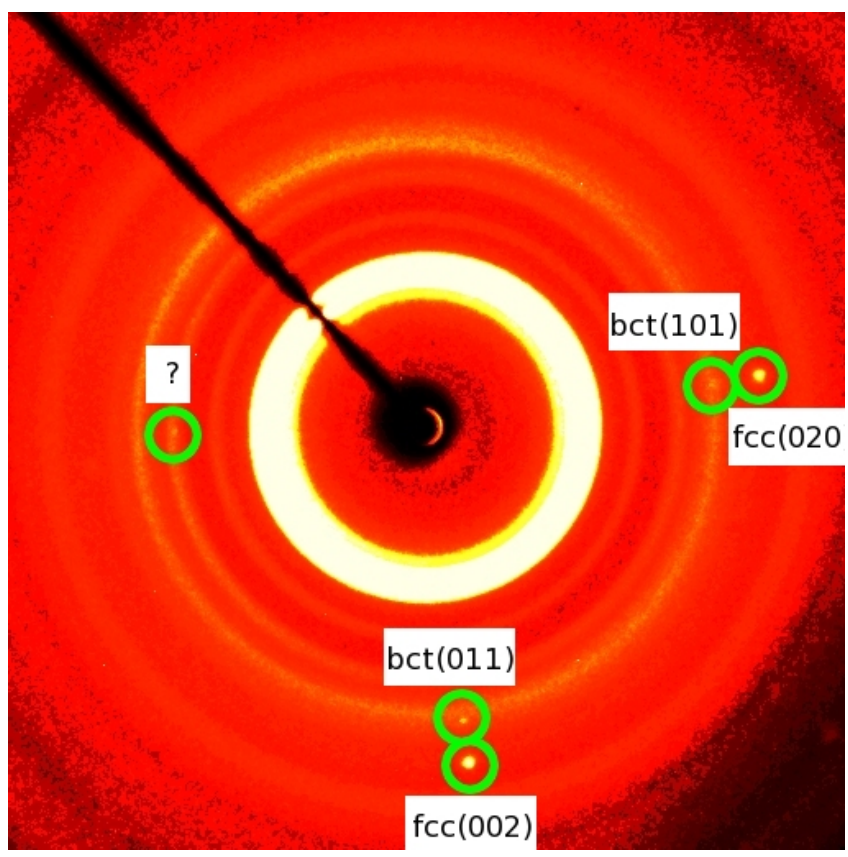

(b) $-100^{\circ} \mathrm{C}$

Abbildung 5.38: Röntgenbeugung in Transmission entlang der Schichtnormalen. Oben bei Raumtemperatur, unten bei $-100^{\circ} \mathrm{C}$. 


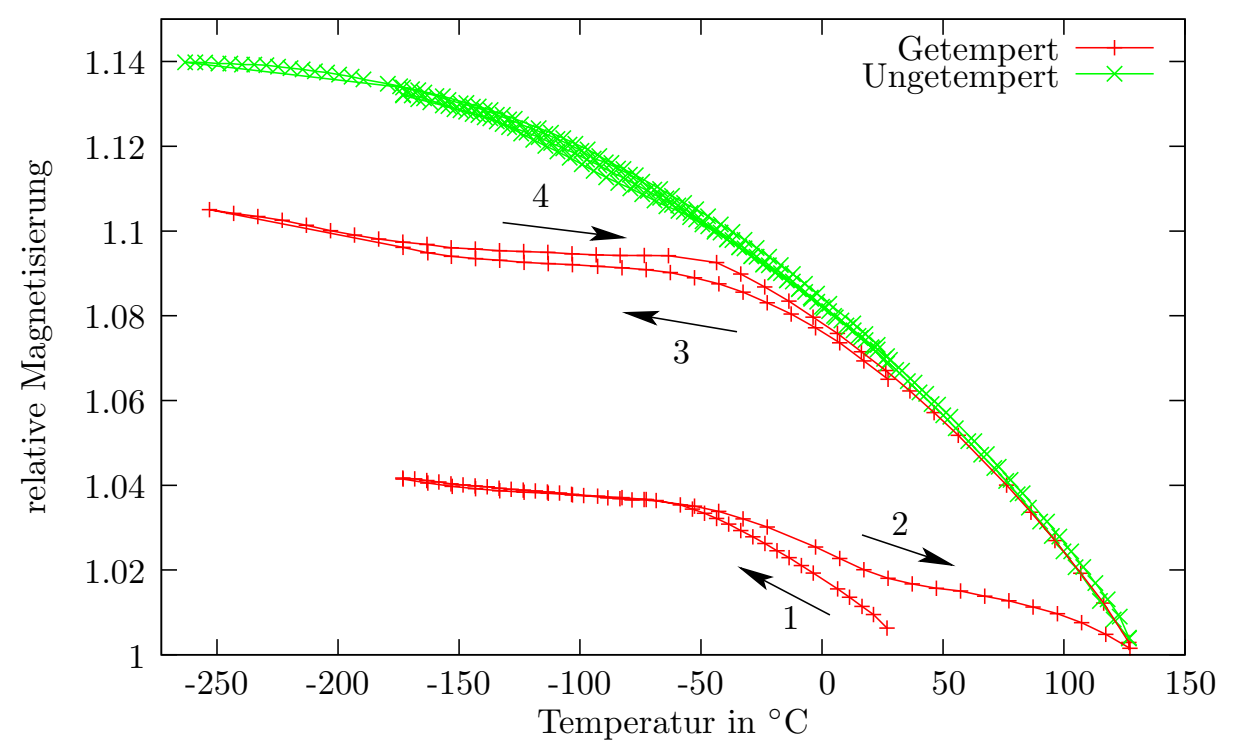

Abbildung 5.39: Magnetisierung einer freistehenden $\mathrm{Fe}_{70} \mathrm{Pd}_{30}$-Probe vor und nach dem Tempern, jeweils gemessen in einem Feld von 100 mT. Aus [111].

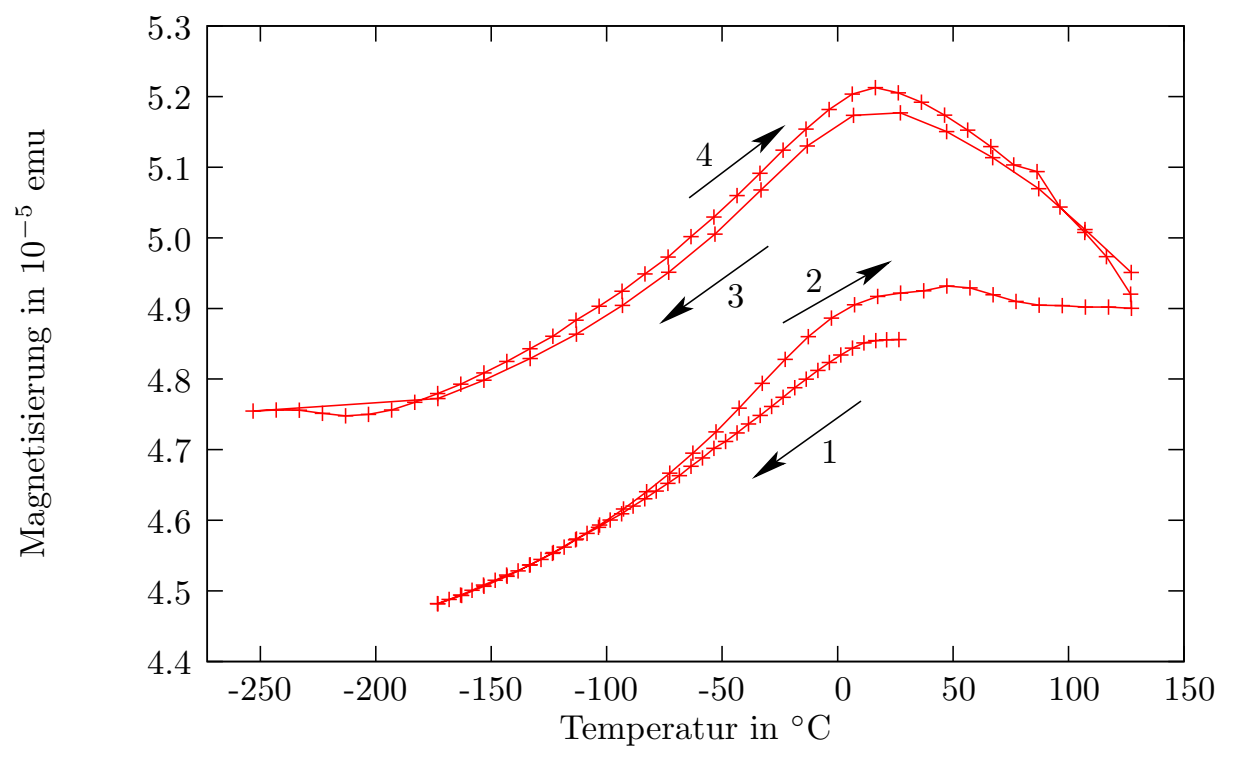

Abbildung 5.40: Magnetisierung einer freistehenden fcc- $\mathrm{Fe}_{72} \mathrm{Pd}_{28}$-Probe nach dem Tempern, gemessen in einem Feld von $100 \mathrm{mT}$. 
somit insgesamt eine höhere Magnetisierung der Probe. Nun folgende Temperaturzyklen zeigen für hohe Temperaturen den selben Verlauf wie die ungetemperte Probe. Bei niedrigen Temperaturen zeigt sich wie schon im ersten Temperaturzyklus die charakteristische Änderung der Steigung beim Martensitübergang.

Eine ebenso präparierte freistehende fcc- $\mathrm{Fe}_{72} \mathrm{Pd}_{28}$-Probe zeigt in Abbildung 5.40 einen qualitativ sehr ähnlichen Verlauf der Magnetisierung, bei der die Abweichung vom Verlauf bei hohen Temperaturen noch deutlicher zu sehen ist. Hier steigt schon unterhalb von etwa $300 \mathrm{~K}$ die Magnetisierung nicht weiter an, sondern fällt sogar wieder unter den Wert bei Raumtemperatur. Die Ursache dieser Änderungen der Magnetisierung liegt in der veränderten Anisotropie der Probe, und wird in Kapitel 6.5 diskutiert. 


\section{Kapitel 6}

\section{Diskussion}

In diesem Kapitel sollen die in den vorherigen Kapiteln dargestellten Ergebnisse der verschiedenen Untersuchungen zueinander in Beziehung gesetzt und interpretiert werden. Dafür werden zunächst die Strukturen, die sich in den Schichten bei den verschiedenen Depositionsmethoden während des Wachstums einstellen, miteinander verglichen und die Ursachen der beobachteten Unterschiede diskutiert. Da die Spannungen beim Schichtwachstum eine entscheidende Rolle spielen, werden diese im Anschluss in einem separaten Abschnitt diskutiert, in dem anhand der Molekulardynamiksimulationen auch der Einfluss von Defekten aufgezeigt wird. Daran schließt sich eine Diskussion der Prozesse an, die in den verschiedenen Klassen von Proben (polykristalline Filme, epitaktische Filme vor und nach dem Ablösen vom Substrat) bei einer Nachbehandlung durch Ionenbestrahlen und Tempern ablaufen, bei denen insbesondere der Defektzustand der Probe verändert wird. Hierbei wird besonders auf den Einfluss des Substrats in epitaktischen Schichten eingegangen, indem solche Proben mit anderen Schichten verglichen werden, die vor dem Tempern vom Substrat abgelöst wurden. Den Abschluss bilden zwei Abschnitte über die mechanischen Eigenschaften des Kristallgitters freistehender Filme, und die strukturellen Änderungen, die sich aus dem sehr weichen Kristallgitter ergebenen.

\subsection{Kristallstruktur der Filme während des Wachstums}

Während des Wachstums der Filme wird die Kristallstruktur stark von der Substrattemperatur, gegebenenfalls zusätzlichen Beiträgen der kinetischen Energie der auftreffenden Metallatome bzw. Ionen und der Kristallstruktur der darunterliegenden Atomlage bestimmt. 
Aus Vorarbeiten ist bekannt, dass $\mathrm{Fe}_{70} \mathrm{Pd}_{30}$-Schichten, die bei Raumtemperatur auf $\mathrm{SiO}_{2}$ aufgedampft werden, in einer sehr feinkörnigen bct-Struktur aufwachsen [33]. Das amorphe $\mathrm{SiO}_{2}$ auf dem Substrat beeinflusst somit die Struktur des darauf wachenden Films nur über die Grenzflächenenergie, die zum Inselwachstum führt.

Die Untersuchungen in dieser Arbeit zeigen, dass sowohl durch eine erhöhte Substrattemperatur als auch durch eine zusätzliche Bestrahlung die hier ablaufenden Prozesse deutlich verändert werden können. Dies führt im Fall höherer Substrattemperaturen zu rauen, entmischten Schichten. Bei der ionenstrahlunterstützten Deposition erhält man dagegen sehr glatte Filme, in denen über einen Konzentrationsbereich von einigen Prozent sowohl die fcc als auch die bcc-Struktur stabil ist. Im Gegensatz dazu kann auf geeigneten kristallinen Substraten, insbesondere auf $\mathrm{MgO}(100)$-Oberflächen sowohl beim Aufdampfen, als auch bei gepulster Laserdeposition unter geeigneten Bedingungen ein epitaktisches Wachstum beobachtet werden.

\subsubsection{Bei hohen Temperaturen auf $\mathrm{SiO}_{2}$ aufgedampfte Filme}

Findet das Wachstum bei höheren Temperaturen statt, wird dadurch im Wesentlichen die Beweglichkeit der Adatome auf dem Substrat erhöht. Insgesamt werden die Schichten dadurch deutlich rauer, da die Grenzflächenenergie dahingehend wirkt, dass die Kontaktfläche zwischen Film und Substrat minimiert wird. Dies führt zu hohen Inseln und tiefen Gräben, die bis auf das Substrat reichen. Innerhalb dieser Inseln ist die Beweglichkeit der Atome offensichtlich hoch genug, dass sich die aus dem Phasendiagramm (Abbildung 2.1) zu erwartenden Gleichgewichtsphasen einstellen. Man beobachtet ein deutliches Entmischen, das sich am Auftreten des bcc(110)-Peaks von Eisen und auch des (111)-Peaks von Palladium festmachen lässt. EDX-Linescans zeigen keine räumlichen Inhomogenitäten, was darauf hindeutet, dass sich eher eine schichtartige Separation einstellt. Betrachtet man die Oberflächenenergien von elementarem Eisen und Palladium, wäre eine Palladium-Terminierung der Oberfläche zu erwarten [112]. XPS-Untersuchungen deuten darauf hin, dass der Oberflächenbereich etwas an Palladium angereichert ist, aber bei einer Schicht, die längere Zeit an Luft gelagert wurde, ist in XPS-Untersuchungen auch Eisenoxid festzustellen. Da bei XPS die oberen $10 \mathrm{~nm}$ der Probe zum Signal beitragen, kann dies entweder auf eine unvollständige Bedeckung der Oberfläche mit Palladium oder eine Diffusion des Sauerstoffs durch die Palladiumoberfläche hindeuten. 


\subsubsection{Ionenstrahlunterstützte Deposition}

Die ionengestützte Deposition erlaubt es, die an der Oberfläche ablaufenden Prozesse unabhängig von der Temperatur des Volumens zu verändern. Hierbei konnte aus den experimentellen Ergebnissen ein Phasendiagramm bestimmt werden (siehe in Abbildung 5.6), in dem über einen recht breiten Zusammensetzungsbereich zwischen 26 und 32 Atomprozent Palladium gleichzeitig sowohl die fcc- als auch die bcc-Struktur beobachtet wird.

Dies deutet darauf hin, dass die treibende Kraft für eine Umwandlung der Phasen ineinander gering sein muss, da beide Phasen ein ähnliches spektrales Gewicht aufweisen, daher also in ähnlichen Volumenanteilen vorliegen. Allerdings steht dies zunächst in deutlichem Widerspruch zu den Ergebnissen ohne Ionenbestrahlung, bei denen bei Raumtemperatur immer nur eine Phase vorliegt. Aus diesen Untersuchungen ohne Ionenbestrahlung ist ebenfalls klar, dass eine einmal bei Raumtemperatur vorliegende Phase hinreichend stabil ist, dass auch bei jahrelanger Lagerung die Filme weder entmischen noch sich in eine andere Gitterstruktur umwandeln. Im Laufe dieses Kapitels wird sich herausstellen, dass die veränderten Bedingungen bei der Keimbildung im Frühstadium des Schichtwachstums diese Koexistenz ermöglichen.

Von der Ionenenergie von $100 \mathrm{eV}$ kann wegen der Impulserhaltung nur ein Teil an ein vor den Stoß ruhendes Targetatom übertragen werden. Durch einen solchen Stoß kann ein Metallatom zunächst nur auf das Substrat zu beschleunigt werden. Erst durch weitere Stöße mit anderen Targetatomen kann die Richtung des Atoms so verändert werden, dass es sich vom Substrat entfernt. Bei jedem dieser Stöße reduziert sich jedoch die Energie des Atoms, so dass zu erwarten ist, dass Sputterprozesse, bei denen Material aus der Schicht entfernt wird, im Vergleich zu Prozessen, bei denen die Atome nach dem Stoß auf dem Substrat redeponiert werden nur eine geringe Rolle spielen. Beim Großteil der Stoßprozesse ist von einem Energieübertrag von wenigen Elektronenvolt auszugehen, so dass nur sehr lokal einige Atome kurzzeitig eine erhöhte Beweglichkeit aufweisen. Aufgrund der geringen Eindringtiefe ist ein durch Channeling verursachte präferentielles Sputtern bestimmter Kristallorientierungen, das zu einer Texturierung der Schicht führen würde, ebenfalls nicht zu erwarten.

Innerhalb der mit dem experimentellen Aufbau zugänglichen Parameter von einer Dekade im Ionenstrom und etwa einer halben Dekade in der Ionenenergie zeigt sich keine deutliche Abhängigkeit der gebildeten Strukturen vom Verhältnis von Depositionsrate und Fluenz des Ionenstrahls. Da keine solche Abhängigkeit gefunden wird, kann ausgeschlossen werden, dass sich die Bestrahlungsparameter exakt im Übergangsbereich zwischen der Stabilität der Phasen bewegen. Auch räumliche Inhomogenitäten, in dem Sinne 
dass auf Teilen der Probenfläche durch eine höhere Dosis eine andere Phase gebildet wird als an anderen Stellen, können ausgeschlossen werden. Vielmehr ist davon auszugehen, dass unter diesen Wachstumsbedingungen die Energiedifferenz zwischen der fcc- und der bcc-Struktur so gering ist, dass Keime beider Gitterstrukturen stabil sind. Werden später weitere Atome auf diese Keime deponiert, ist die Beweglichkeit dieser neu deponierten Atome hinreichend groß, dass sie sich gemäß der jeweiligen lokal vorhandenen Kristallstruktur anordnen.

Dies deutet darauf hin, dass durch die Ionenbestrahlung wie auch bei vielen anderen Systemen beobachtet, in der Anfangsphase des Wachstums eine größere Anzahl kleinerer Keime gebildet werden [69, 71]. Die verwendete Ionenenergie ist hoch genug, um einzelne Atome aus den Flanken der vorhandenen Keime wieder zu entfernen $[113,114]$. Diese Atome werden nach einem solchen ballistischen Stoß mit einem Argonion mit hoher Wahrscheinlichkeit auf das Substrat redeponiert, und bilden dort neue Keime. Dieser als ballistischer Transport bezeichnete Mechanismus glättet die Schicht auch in späteren Phasen des Wachstums dadurch, dass Atome von den Hügelflanken in die Täler transportiert werden. Dies erklärt somit auch, warum sowohl in den elementaren wie auch in den Legierungsfilmen die RMS-Rauigkeit nur etwa halb so groß groß ist wie in Vergleichsschichten ohne Ionenbestrahlung.

Da durch die Ionenbestrahlung mehr und dafür kleinere Keime gebildet werden, bedecken diese bei gleicher Materialmenge einen größeren Teil des Substrats als wenige höhere Inseln. Dadurch wird viel früher eine vollständige Bedeckung des Substrats erreicht, wonach der Einfluss der Grenzflächenenergie zwischen Film und Substrat nicht mehr zum Aufrauen der Filme führen kann. Im Vergleich zu Proben, die ohne Ionenbestrahlung aufgedampft wurden, sind die Röntgen-Reflexe der Proben deutlich verbreitert und weniger hoch, was ebenfalls auf eine sehr feinkörnige Struktur hinweist [115].

Bei Schichten, die auf $\mathrm{SiO}_{2}$ aufgedampft werden, wird in der fcc-Struktur hauptsächlich eine (111)-Drahttextur beobachtet, während die bcc-Struktur eine (110)-Drahttextur aufweist. Ein Vergleich der Ebenen, die bei diesen Texturen parallel zum Substrat verlaufen in Abbildung 6.1 zeigt, dass sie einander sehr stark ähneln, und sich durch eine Dehnung ineinander überführen lassen. Die fcc(111)-Ebenen weisen die sechszählige Symmetrie einer hexagonalen Struktur auf, die in einer bcc(110)-Ebene gebrochen ist, die nur zwei Spiegelsymmetrien aufweist, deren Symmetrieachsen senkrecht aufeinander stehen. Insbesondere bei kleinen Keimen ist es leicht vorstellbar, dass die Struktur leicht deformiert ist, da hier die Ränder eines Bereichs einen großen Einfluss haben können. So ist es durchaus möglich, dass sich Keime in beiden Strukturen bilden. Auch quantenmechanische Rechnungen zeigen, dass sich $\mathrm{Fe}_{70} \mathrm{Pd}_{30}$ sehr leicht von der fcc bis zur bcc Struktur dehnen lässt [15, 13]. 


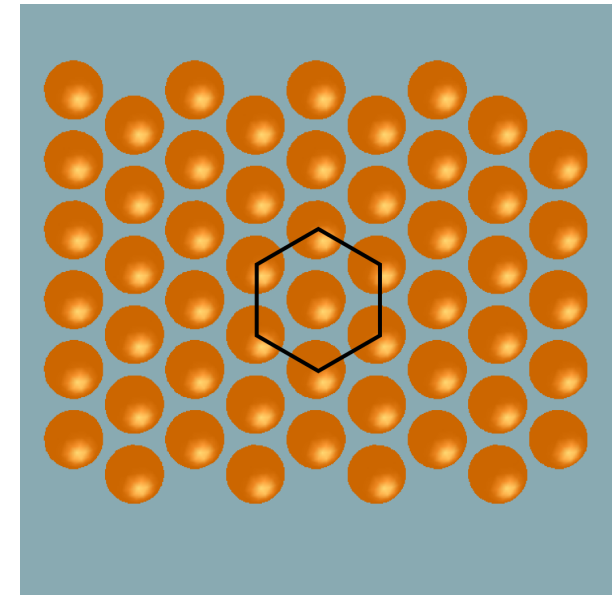

(a) fcc(111)-Ebene

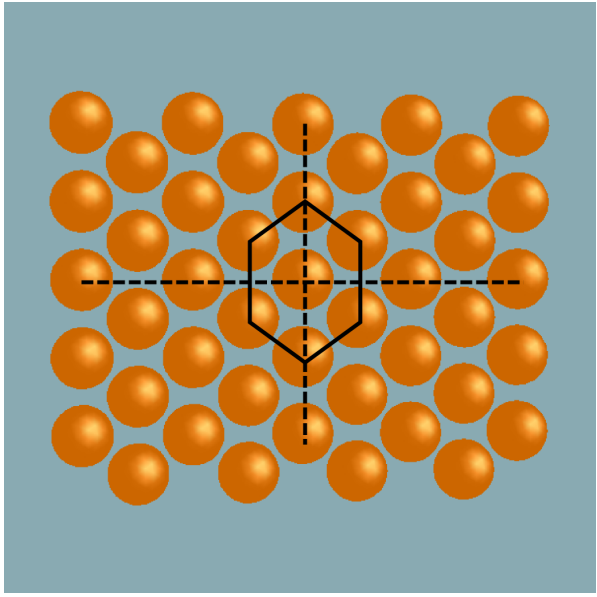

(b) bcc(110)-Ebene

Abbildung 6.1: Die fcc(100)-Ebene weise eine sechszählige Symmetrie auf. Die bcc (100)-Ebene ähnelt ihr sehr, hat aber nur zwei Spiegelsymmetrien.

Bei der Deposition bei höheren Temperaturen wird dies nicht beobachtet, da diese entweder zum Entmischen führen, oder bei noch höheren Temperaturen nur die fcc-Phase stabil ist (siehe Phasendiagramm in Abbildung 2.1)

In kleineren Keimen ist darüber hinaus auch die Stöchiometrie nicht immer sichergestellt, da schon wenige Atome die Zusammensetzung deutlich verschieben können. Auch dadurch ließe sich plausibel erklären, dass sich in der Anfangsphase des Wachstums sowohl fcc- als auch bcc- Keime bilden.

Sobald ein Keim nun von weiteren Atomen überwachsen wird, wird dadurch seine Kristallstruktur zusätzlich stabilisiert. Die Energieunterschiede zwischen den verschiedenen Gitterstrukturen sind sehr gering [15, 13], und reichen offensichtlich nicht aus, um eine Umwandlung in eine andere Gitterstruktur zu bewirken. Dies bedeutet, dass die Energiedifferenz nicht ausreicht, um die für eine Umwandlung notwendige elastische Energie aufzubringen, da alle umgebenden Körner bei einer Umwandlung deutlich verzerrt würden. Dies ist in einzelnen, stärker voneinander getrennten Inseln, wie sie sich ohne Ionenbestrahlung bilden, sehr viel leichter als in einer durch die Bestrahlung geglätteten Struktur. So stabilisieren sich die einzelnen Strukturen durch die mit der geringen Größe und der geringen Rauheit verbundene hohe Zahl an Korngrenzen jeweils gegenseitig. Durch die geringe Energie des Ionenstrahls ist ein Einfluss der Ionen auf tieferliegende Teile des Films auszuschließen.

Wird während der ionenunterstützten Deposition zusätzlich das Substrat geheizt, beobachtet man für Substrattemperaturen von 300,400 und $500^{\circ} \mathrm{C}$ 
ein Entmischen der Filme, genau wie es bei diesen Temperaturen auch ohne Ionenbestrahlung auftritt. Auch hier spiegelt sich wieder, dass die Eindringtiefe der Ionen nicht groß genug ist um das Volumen des Films hinreichend zu durchmischen, wie es bei der Bestrahlung mit sehr viel höheren Ionenenergien zu erwarten wäre. Somit ist klar, dass hier zwischen Oberflächenprozessen, insbesondere der Phasenselektion beim Wachstum, und Volumenprozessen, insbesondere Diffusion unterschieden werden muss, und sich deren jeweiliges Gewicht mittels der ionenunterstützten Deposition deutlich gegeneinander verschieben lässt.

\subsubsection{Gepulste Laserdeposition}

In einem gewissen Sinne stellt auch die gepulste Laserdeposition ein der ionenstrahlunterstützten Deposition sehr ähnlichen Prozess dar, bei dem allerdings die deponierten Atome selbst kinetische Energien haben, die weit über der thermischen Energie verdampfender Metallatome liegen. Ein Hauptunterschied zur ionengestützten Deposition besteht im Verbleib der energiereichen Atome: Während bei der PLD die energiereichen Metallatome nach einigen Kollisionen im Film als Zwischengitteratome verbleiben, verlässt bei der ionenstrahlunterstützten Deposition ein sehr großer Teil der Argonatome die Schicht wieder, da sie keine chemische Bindung mit den Metallatomen eingehen. Mittels der PLD werden (abgesehen von den Droplets) sehr glatte Filme produziert, in denen sich ebenfalls Phasen stabilisieren lassen, die nicht dem thermischen Gleichgewicht entsprechen. Hier beobachtet man bei einem Palladium-Gehalt von 30 Prozent und darüber Filme in der fcc bzw. fct-Struktur, die zunächst epitaktisch mit einer (100)\|(100); [001]||[001]Orientierung auf dem MgO-Substrat aufwachsen und dabei sehr stark elastisch verspannt sind [35, 103]. Diese Verspannung hat einen großen Einfluss auf das weitere Schichtwachstum und wird in Kapitel 6.2.1 eingehend diskutiert. Für Filme mit geringerem Palladiumgehalt wird dagegen beobachtet, dass hier die bcc-Struktur in einer (100) ||(100); [001]||[011]-Orientierung wächst [35].

Neuere Untersuchungen von Buschbeck et. al. an sehr dünnen Filmen zeigen, dass sich durch die Wahl geeigneter Zwischenschichten die vom Misfit zwischen Substrat und Film verursachte Verspannung beeinflussen lässt, während gleichzeitig die Epitaxie erhalten wird. Hierbei lassen sich in sehr dünnen Schichten von einigen Nanometern fast alle Achsenverhältnisse des Bain-Pfades realisieren [13]. 


\subsubsection{Epitaktisches Aufdampfen}

Treffen die Atome dagegen beim Aufdampfen nur mit geringer Energie auf ein $\mathrm{MgO}(100)$-Substrat, so ist oberhalb von $500^{\circ} \mathrm{C}$ ein epitaktisches Wachstum der fcc-Struktur in einer (100)\|(100); [001]\|[001]-Orientierung zu beobachten. Darüber hinaus treten bei Temperaturen unterhalb von $690^{\circ} \mathrm{C}$ bcc-Eisenausscheidungen auf, die jedoch die Epitaxie nicht vollständig zusammenbrechen lassen. Hierbei ist der Zustand der Oberfläche der $\mathrm{MgO}-$ Substrate von kritischer Bedeutung: Durch die Lagerung der Substrate bildet sich durch die in der Luft immer in geringen Mengen vorhandene Feuchtigkeit an der Oberfläche eine Magnesiumhydoxid-Schicht [116], die das Wachstum empfindlich stört, und zur vermehrten Bildung von Eisenausscheidungen führen kann [105]. Diese Schicht kann jedoch durch eine Temperaturbehandlung der Substrate vor der Deposition entfernt werden, in dem die Substrate bereits 90 Minuten vor dem Beginn der Deposition auf die Depositionstemperatur gebracht werden, so dass zum einen mögliche Adsorbate abdampfen, und zum anderen Oberflächendefekte von der Herstellung der Substrate ausheilen können. Es ist somit möglich, auf $\mathrm{MgO}$ bei einer Substrattemperatur von $690^{\circ} \mathrm{C}$ epitaktische Filme herzustellen, die keine Eisenausscheidungen aufweisen, während Filme, die bei dieser Temperatur auf thermisch oxidiertes Silizium aufgedampft wurden entmischen. Beim epitaktischen Wachstum zwingt das Substrat zunächst dem Film seine Gitterstruktur auf. Hierbei ist die Gitterfehlpassung und die daraus resultierende mechanische Spannung von entscheidender Bedeutung, die im folgenden Kapitel diskutiert werden. Wie sich in den Molekulardynamiksimulationen in Kapitel 5.3 zeigt, behindern Eisenausscheidungen den Abbau der Verspannung, so dass ihre Bildung schon bei niedrigeren Temperaturen unterdrückt wird, als dies im Bulk-Phasendiagramm beobachtet wird.

\subsection{Mechanische Spannungen}

Im Anfangsbereich des Schichtwachstums, während die Epitaxie noch strikt gewährleistet ist, wird der Film durch die Fehlpassung zwischen den Gitterkonstanten des Filmmaterials und des Substrats gedehnt. Mit wachsender Filmdicke nimmt die elastische Energie dieser Verzerrung immer weiter $\mathrm{zu}$, und führt dazu, dass verschiedene Relaxationsmechanismen diese Energie reduzieren. Wachsen die Filme cube-on-cube auf (wie z. B. Filme in der fccStruktur auf $\mathrm{MgO}(100)$-Oberflächen), so können direkt die Gitterkonstanten 
von Film und Substrat verglichen werden, um die Fehlpassung $f$ zu erhalten:

$$
f=\frac{a_{\mathrm{MgO}}-a_{\mathrm{FePd}}}{a_{\mathrm{MgO}}}
$$

In der bcc-Struktur sind die Einheitszellen zum Substrat um $45^{\circ}$ gedreht, so dass hier der Faktor $\sqrt{2}$ auftritt:

$$
f=\frac{a_{\mathrm{MgO}}-\sqrt{2} \cdot a_{\mathrm{FePd}}}{a_{\mathrm{MgO}}}
$$

Für $\mathrm{Fe}_{70} \mathrm{Pd}_{30}$-Filme in der fcc-Struktur lässt sich die Spannung, die notwendig ist, um den Film bis zur Gitterkonstante von $\mathrm{MgO}$ zu dehnen, zu etwa

$$
\sigma=\frac{E}{1-\nu} \cdot f \approx 20 \mathrm{GPa}
$$

abschätzen, wenn man für die Dehnung $f$ einen Wert von 0,105 ansetzt, und als Abschätzung für die Poissonzahl $\nu=1 / 3$ und den E-Modul $E=120 \mathrm{GPa}$ verwendet [16]. Dieser Wert wird in keinem der Experimente beobachtet, da die Spannungen auf verschiedene Arten, abhängig von den Bedingungen der Deposition, relaxieren.

\subsubsection{Bei Raumtemperatur laserdeponierte Schichten}

Allen mittels PLD hergestellten Filmen ist es gemein, dass sie in der Spätphase des Filmwachstums druckverspannt sind. Dies ist für solchermaßen abgeschiedene Filme nicht ungewöhnlich $[117,118,119,120]$, und wird auch bei anderen Depositionsmethoden beobachtet, bei denen Atome mit hoher kinetischer Energie auf das Substrat treffen wie beim Sputtern [56] oder Ionenbestrahlen [121]. Bei der hohen Energie der deponierten Atome von bis zu $100 \mathrm{eV}$ [74] ist damit zu rechnen, dass in den schon deponierten Schichten Punktdefekte, vor allem Zwischengitteratome, entstehen. Dieser Effekt wird als Shot Peening bezeichnet. Positive Bildungsvolumina für Zwischengitteratome können so zu einer Verspannung der Schicht führen.

Reines Eisen wächst über den gesamten betrachteten Dickenbereich epitaktisch auf $\mathrm{MgO}$, durch die Fehlpassung von $3 \%$ wären hierbei Zugspannungen zu erwarten. Tatsächlich beobachtete man aber von Beginn des Wachstums an Druckspannungen, was nahe legt, dass hier die Fehlpassung durch das oben bereits diskutierte Shot Peening überkompensiert wird. Für den $\mathrm{Fe}_{80} \mathrm{Pd}_{20}$-Film wären noch geringere Zugspannungen durch die Fehlpassung von nur 0,6 \% zu erwarten, so dass in diesem Film die in der Anfangsphase beobachteten Druckspannungen höher sind als in der reinen Eisenschicht, da hier geringere Zugspannungen kompensiert werden müssen. 
Für diese beiden Filme, die eine kubische Struktur aufweisen, wurde mit Hilfe der $\sin ^{2} \Psi$-Methode ebenfalls die Verzerrung der jeweiligen Gitterzelle gemessen, wie in Abbildung 5.14 dargestellt. Die aus den Ebenenabständen berechneten Gitterkonstanten folgen recht gut einem linearen Verlauf, so dass sich daraus die Verzerrung der Zellen bestimmen lässt. Berechnet man hieraus die Spannung, die notwendig ist, um solch eine Verzerrung zu erreichen, erhält man Druckspannungen, deren Werte von den in-situ gemessenen Spannungen deutlich abweichen. Dies ist nicht ungewöhnlich, so wurde in Messungen an ionenbestrahlten Wolfram-Filmen Abweichungen um den Faktor zwei beobachtet [122], bei der Bestrahlung von Stahl wurden auch schon Abweichungen um den Faktor 9 festgestellt [123, 124].

Der Grund für diese Abweichungen liegt darin, dass die Messung der Substratkrümmung immer über den gesamten Film mittelt, während bei Röntgenstreumethoden nur der Teil des Probenvolumens ein Signal liefert, an dem die Röntgenstrahlung kohärent gestreut wird. Trotz des epitaktischen Wachstums beträgt die Kohärenzlänge dieser Filme nur etwa $20 \mathrm{~nm}$ [35], so dass klar wird, dass ein nicht unerheblicher Teil des Filmvolumens zu Bereichen mit gestörter Kristallstruktur wie der Umgebung von Punktdefekten oder Korngrenzen gehört, und damit zu den aus kohärenter Röntgenstreuung gewonnenen Spannungen nicht beiträgt. Eben diese Bereiche sind es allerdings, in denen ein großer Teil der Spannungsrelaxation zu erwarten ist, so dass vor diesem Hintergrund der Unterschied der aus der Substratkrümmungsmessung und aus der $\sin ^{2} \Psi$-Methode bestimmten Werte plausibel erscheint. Darüber hinaus ist zur Bestimmung der Spannung aus der Verzerrung der Gitterzelle die Kenntnis des elastischen Moduls der Schicht notwendig. Dieser kann unter Umständen stark von der Schichtdicke (und damit dem Oberfläche-zuVolumenverhältnis), der Mikrostruktur oder der Defektdichte abhängen, so dass der Rückgriff auf Moduli, die an Bulk-Proben bestimmt wurden ebenfalls fragwürdig erscheint. Wegen der geringen Tendenz zur Oxidation kann dagegen eine Veränderung der Probe zwischen der in-situ Messung der Substratkrümmung und der ex-situ Röntgenbeugung weitgehend ausgeschlossen werden.

Die Filme mit $30 \%$ und $40 \%$ Palladium weisen eine fct-Struktur auf, bei der die lineare Elastizitätstheorie zur Bestimmung der Spannung aus der Dehnung nicht angewendet werden kann. Hier ist davon auszugehen, dass durch die Verspannung der Martensitübergang ausgelöst wird, um die elastische Energie der Filme zu minimieren. In Bulk-Proben wurden für tiefe Temperaturen tetragonale Verzerrungen von bis zu $1-c / a=6 \%$ gemessen [36], was nicht ausreicht, um die Gitterfehlpassung zwischen Film und Substrat vollständig zu kompensieren, so dass zu erwarten wäre, dass die beiden längeren $a$-Achsen (Fehlpassung $9 \%$ ) in der Schichtebene liegen, und die kürzere 

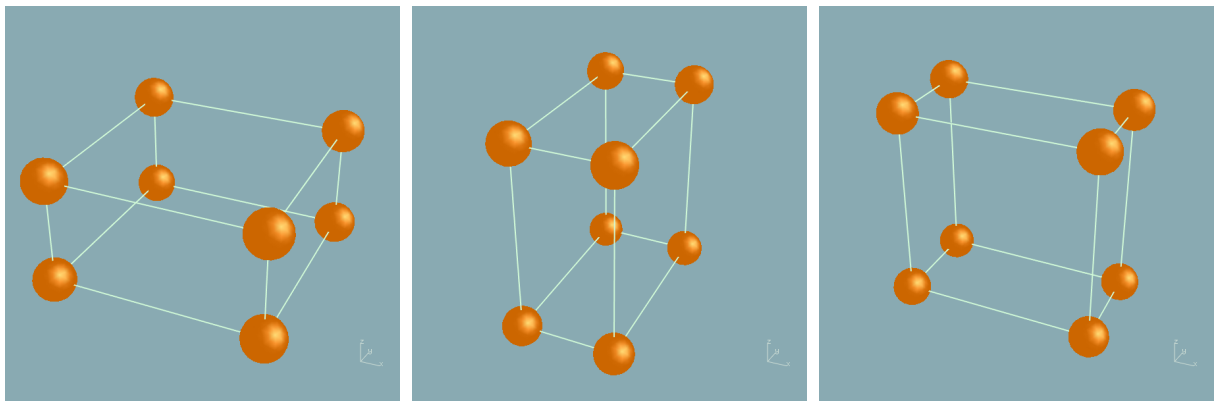

Abbildung 6.2: Die drei möglichen Anordnungen einer Gitterzelle mit zwei langen $a$-Achsen und einer kurzen $c$-Achse.

c-Achse (Fehlpassung $12 \%$ ) senkrecht zur Schichtebene liegt (wie in der linken Darstellung in Abbildung 6.2). Frühere Messungen zeigten, dass bei vergleichbaren Schichten in der fct-Phase nur der im Vergleich zur fcc-Struktur zu kleineren Winkeln hin verschobene (200)-Peak, und nicht der (002)-Peak auftritt [35]. Dies bedeutet, dass immer eine lange Achse senkrecht zur Substratebene liegt, und steht zunächst im Widerspruch zur Erwartung, da die Gitterfehlpassung deutlich kleiner wäre, wenn beide langen Achsen in der Ebene lägen. Diese Messungen wurden allerdings an Schichten durchgeführt, deren Dicke weit über $20 \mathrm{~nm}$ liegt. Hierbei ist davon auszugehen, dass der größte Teil der Gitterzellen nicht durch die Gitterfehlpassung zum Substrat, sondern durch das später dominierende shot-peening ausgerichtet werden.

Außer einer bevorzugten Orientierung der Martensitvarianten wird auch eine spannungsinduzierte (111)-Verzwillingung beobachtet, deren vielfaches Auftreten schließlich zum Zusammenbruch der Epiataxie führt [103]. Danach überwiegen die Druckspannungen, die durch die Zwischengitteratome ausgelöst werden. Somit ist eine Anordnung mit beiden langen Achsen in der Ebene sehr ungünstig, so dass nun die Anordnungen, die eine kurze Achse in der Filmebene haben, zu erwarten sind. Dieser Mechanismus kann jedoch die auftretenden Spannungen nicht kompensieren, so dass die Krümmungsmessungen einen sehr deutlichen Anstieg zeigen. Dies ist dadurch zu begründen, dass je nach Art der Spannungen diese nur teilweise oder gar nicht über den Mechanismus der Reorientierung der Martensitvarianten kompensiert werden können: Bei einer uniaxialen Druckspannung gibt es genau eine Anordnung der Varianten, die energetisch am günstigsten ist. Diese ist dadurch charakterisiert, dass alle kurzen Achsen in Richtung der Druckspannung ausgerichtet sind. Biaxiale Druckspannungen können nicht vollständig kompensiert werden, werden jedoch zu einer Anordnung führen, bei der die zwei Varianten, 
deren kurze Achse in der Ebene der Druckspannungen liegt, die Spannungen teilweise kompensieren. Wird das Material jedoch aus allen Raumrichtungen komprimiert, wie es bei der Erzeugung von Zwischengitteratomen deutlich unter der Schichtoberfläche geschieht, kann diese Spannung durch keine Anordnung der verschiedenen Varianten kompensiert werden.

\subsubsection{Bei hohen Temperaturen aufgedampfte Schich- ten}

Im Gegensatz zu den mittels PLD hergestellten Schichten, ist es beim Aufdampfen auf $\mathrm{MgO}$ bei hohen Substrattemperaturen möglich, die Schichten mit sehr viel geringeren Spannungen herzustellen. Der Grund hierfür liegt in der höheren Beweglichkeit der Atome, die es erlaubt, die Spannungen über den Einbau von Versetzungen in den Film abzubauen, und der Abwesenheit des Spannungen erzeugenden Shot Peenings.

Die Schichten, die bei Temperaturen unterhalb von $690^{\circ}$ deponiert wurden, verfügen sogar über einen weiteren Mechanismus, über den die Gitterfehlpassung zwischen Substrat und Film kompensiert werden kann: Die Eisenausscheidungen liegen bevorzugt als flache Platten an der Grenzfläche zwischen Film und Substrat, und können so als Pufferschicht wirken, da die Gitterkonstante von reinem Eisen zwischen der von $\mathrm{MgO}$ und FePd liegt. Trotzdem weist diese Schicht im Vergleich zu anderen Depositionstemperaturen die höchsten Spannungen auf. In diesen Schichten wird hauptsächlich eine Spannungsrelaxation über 1/6 $\langle 112\rangle$-Shockley-Partialversetzungen beobachtet $[34,105]$, wie sie auch in $\mathrm{Fe}_{50} \mathrm{Pd}_{50}$ dominiert $[125,126]$. In den Molekulardynamiksimulationen lässt sich dieser Mechanismus deutlich beobachten: In der Simulationszelle ohne Eisenausscheidungen bilden sich ab einer kritischen Dehnung von etwa 4.5\% (siehe Abbildung 6.3 und Abbildung 5.21) zunächst zwei Partialversetzungen an der Oberfläche, die dann mit einer Geschwindigkeit von etwa 1600 Metern pro Sekunde entlang einer (111)-Ebene durch die Schicht laufen, bis sie auf das Substrat treffen. Später laufen dann weitere Partialversetzungen über die angrenzenden (111)-Ebenen, die nun ihrerseits keine neuen Stapelfehler mehr erzeugen, sonder nur den Bereich zwischen den beiden Stapelfehlern verbreitern, und so diesen Mikrozwilling wachsen lassen. Die Simulationszelle mit den Eisenausscheidungen zeigt ein qualitativ ähnliches Verhalten, allerdings ist hier eine deutlich höhere kritische Dehnung von 4.7\% notwendig, bevor die Zwillingsbildung einsetzt, und auch der Biaxailmodul (die Steigung der Spannungs-Dehnungs-Kurve im linearen Bereich) liegt in der Zelle mit den Ausscheidungen mit 238,6 \pm 0,4 GPa deutlich über dem der Zelle ohne Eisenausscheidungen, der 192,6 $\pm 0,5 \mathrm{GPa}$ 


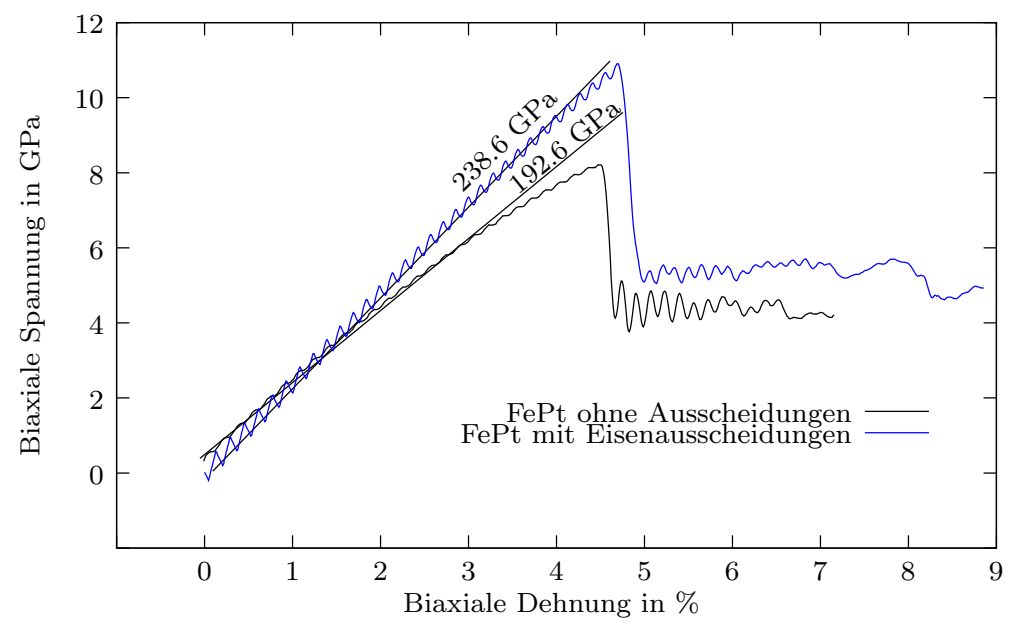

Abbildung 6.3: Spannungs-Dehnungs-Kurven aus den MD-Simulationen. Die Zelle mit den Eisenausscheidungen zeigt sowohl einen höheren Biaxialmodul als auch eine höhere kritische Spannung, bevor die Verzwillingung einsetzt.

beträgt. Diese Werte lassen sich jedoch nicht direkt auf die Experimente übertragen, da das verwendete Potential eine zu hohe Energie für die Verzwillingung liefert (siehe die Diskussion der elastischen Konstanten in [95]).

Unabhängig von einer quantitativen Übereinstimung zwischen Simulationen und Experimenten lässt sich jedoch deutlich erkennen, dass die Eisenausscheidungen einen negativen Effekt auf den Spannungsabbau in der Schicht haben, in dem sie einzelne Partialversetzungen pinnen, so dass Mikrozwillinge am Wachsen gehindert werden. Dies erklärt die deutlich höheren Spannungen in der bei relativ niedriger Temperatur aufgedampften Schicht in Abbildung 5.16. In den entmischenden Schichten wurden genau solche Mikrozwillinge gefunden, nicht aber in den Schichten, die bei Temperaturen von $690{ }^{\circ} \mathrm{C}$ und darüber hergestellt wurden [34, 105]. Im Gegensatz zu den teilweise entmischten Schichten weisen die nicht entmischten Schichten in TEM-Untersuchungen keine Partialversetzungen auf. Hier dominieren volle Versetzungen, insbesondere in der Nähe der Grenzfläche zwischen Film und Substrat. Eine Detailansicht einer solchen Versetzung ist in Abbildung 6.4 zu sehen.

Betrachtet man den Verlauf der Substratkrümmung der bei $690^{\circ} \mathrm{C}$ in zwei Depositionsphasen hergestellten Schicht in Abbildung 5.16, kann man diese in drei Bereiche einteilen. Zunächst steigt die Krümmung linear an, und ist nach einem Übergangsbereich, in dem ein Maximum auftritt, konstant. Wenn 


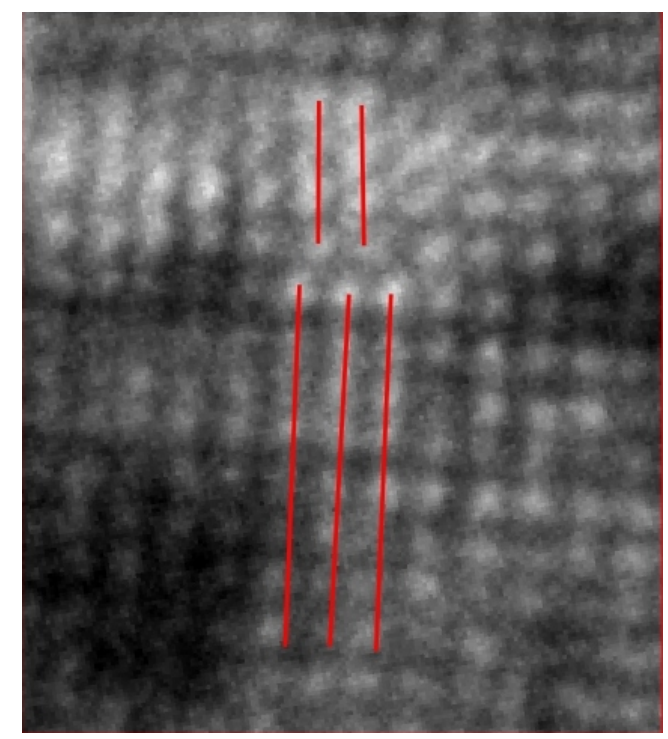

Abbildung 6.4: HRTEM-Detailansicht einer Versetzung in einem $\mathrm{Fe}_{70} \mathrm{Pd}_{30^{-}}$ Film.

keine Relaxationsprozesse im Volumen ablaufen, ist die Spannung der gerade aufgewachsenen Atomlage durch die Ableitung der Krümmung gegeben. Das bedeutet, dass für Schichtdicken bis etwa $60 \mathrm{~nm}$ eine konstante Spannung in der gerade aufwachsenden Schicht herrscht, und ab etwa $100 \mathrm{~nm}$ alle weiteren Atomlagen spannungsfrei aufwachsen. Die Gitterfehlpassung wird somit zum einen durch den kontinuierlichen Einbau von vollen Versetzungen abgebaut, die auch in den Abbildungen 5.15 und 6.4 deutlich zu sehen sind, und zum anderen trägt sicher auch die Oberflächenmorphologie zum Spannungsabbau bei. Da das Substrat zu Anfang nicht vollständig bedeckt ist, sondern einzelne Inseln aufwachsen, können die Atome in den oberflächennahen Bereichen der Inseln eine andere Gitterkonstante aufweisen, als an der Grenzfläche zum Substrat. Kurz vor der Schichtdicke, bei der die Substratkrümmung maximal ist, erreicht die vom Film reflektierte Intensität (siehe Abbildung 5.17) ihr Maximum, der Film ist also hier besonders glatt [127]. Dies deutet darauf hin, dass auch das koaleszieren der Inseln mit der Verspannung der Schicht zusammenhängt. Sobald der Film sich schließt, steht der Mechanismus des Spannungsabbaus an der Oberfläche der Inseln nicht mehr zur Verfügung. Das Schließen der Schicht ist also erst dann energetisch günstig, wenn genügend Versetzungen in der Schicht eingebaut sind, so dass die von der Gitterfehlpassung erzeugte Verspannung abgebaut ist.

Die Deposition dieses Films geschah in zwei Phasen, wie in Kapitel 5.2.2 
dargestellt. Nach dem Ende der Depositionspause ist die Substratkrümmung zunächst deutlich geringer als vor dem Beginn der Unterbrechung, und steigt dann während der Deposition der nächsten $20 \mathrm{~nm}$ wieder auf den ursprünglichen Wert vor der Unterbrechung. Während dieses Anstieges ist die Intensität des reflektierten Laserstrahls in guter Näherung konstant, so dass ein massives Aufrauen, wie es bei einer Asaro-Tiller-Grinfeld-Instabilität auftreten würde [128], als Ursache für diese Krümmungsänderung ausgeschlossen werden kann. Nach dem Ende der zweiten Deposition fällt die Substratkrümmung wieder, und folgt dabei einem Verlauf, der in Größe und Form dem Anstieg zu Beginn der zweiten Deposition gut entspricht, während die Reflektivität weiterhin genau so ansteigt, wie sie es auch schon vor dem Ende der zweiten Depositionsphase tut. Auch hier kann also die Oberflächenmorphologie als Ursache ausgeschlossen werden. Vielmehr ist davon auszugehen, dass sich währen des Wachstums ein dynamisches Gleichgewicht ausbildet, da einerseits beim Wachstum z. B. durch Überwachsen der Oberflächenstruktur Defekte gebildet werden, und andererseits durch die hohe Temperatur im Volumen des Films vorhandene Defekte ausheilen können. Wird die Deposition nun beendet, relaxieren die Defekte im Volumen weiterhin, während keine neuen gebildet werden, so dass insgesamt die Spannungen abnehmen.

Eine so große Änderung der Spannung, wie sie zu Beginn und Ende der zweiten Depositionphase beobachtet wird, wäre bei der schon vorhandenen Schichtdicke für einen reinen Oberflächeneffekt nicht zu erwarten. Darüber hinaus nimmt die Intensität der reflektierten Laserstrahlung auch dann noch weiter zu, wenn Spannung bereits einen konstanten Wert erreicht hat und sich nicht weiter verändert. Dies kann als weiterer Beweis dafür gewertet werden, dass die beobachteten Spannungsänderungen mit Volumen- und nicht nur mit Oberflächenprozessen zusammenhängen, die sich auch nach dem Ende der Deposition weiter fortsetzen. Bei diesen hohen Temperaturen ist durch Diffusion eine hohe Beweglichkeit der Defekte im Volumen zu erwarten, um die Gesamtenergie der Schicht zu minimieren. Wird die Depositionstemperatur nach dem Ende des Aufdampfens noch lange gehalten, wäre so eine Minimierung der Schichtoberfläche zu erwarten, in dem sich die Oberfläche glättet, und wie sie sich auch nach langem nachträglichen Tempern einstellt (siehe Abbildung 5.18).

Die Verspannung der Filme durch unterschiedliche thermische Ausdehnungskoeffizienten von Film und Substrat ist bei einem Material, dessen thermischer Ausdehnungskoeffizient sich stark verändert (siehe Abbildung 5.36), nur schwer zu bestimmen. Messungen zur thermischen Ausdehnung von Bulk$\mathrm{Fe}_{68} \mathrm{Pd}_{32}$-Polykristallen, die je nach Temperatur in der fcc- oder der fct-Phase vorliegen, liefern Werte zwischen $2 \cdot 10^{-6}$ und $12 \cdot 10^{-6} \mathrm{~K}^{-1}$ [129]. Der thermische Ausdehnungskoeffizient von $\mathrm{MgO}$ liegt im Mittel über den beim Tem- 
pern relevanten Temperaturbereich bei etwa $13 \cdot 10^{-6} \mathrm{~K}^{-1}[110]$, und ist damit deutlich größer als der von Siliziumsubstraten, deren Ausdehnungskoeffizient im Mittel bei $3,5 \cdot 10^{-6} \mathrm{~K}^{-1}$ liegt [130]. Die abgelösten Filme, die in Kapitel 6.3.2 diskutiert werden, liegen nur lose auf den $\mathrm{SiO}_{2}$-Substraten auf, so dass die Kräfte in diesen Proben bei nachträglichen Temperaturbehandlungen gering sein werden.

\subsection{Nachträgliche Veränderung der Kristall- struktur}

Die beim Wachstum gebildete Kristallstruktur lässt sich im Nachhinein sowohl durch eine Ionenbestrahlung als auch eine Temperaturbehandlung verändern. In polykristallinen Filmen, in denen das Substrat nur einen geringen Einfluss auf die Struktur des Films ausübt, wurde der Effekt einer hochenergetischen Ionenbestrahlung auf die Gitterstruktur der $\mathrm{Fe}_{70} \mathrm{Pd}_{30}$-Filme untersucht. Unabhängig von der Temperatur, bei der diese Beschuss stattfindet, beobachtet man ein deutliches Kornwachstum. Bei Raumtemperatur bleibt die Schicht dabei in der bct-Struktur, bei erhöhter Substrattemperatur während dem Ionenbeschuss kann sich jedoch auch die fcc-Phase einstellen. Werden solche polykristallinen $\mathrm{Fe}_{70} \mathrm{Pd}_{30}$-Filme getempert, wird ein massives Kornwachstum der fcc-Phase beobachtet. Dagegen kann man in epitaktischen $\mathrm{Fe}_{72} \mathrm{Pd}_{28}$ Filmen, die vor und nach dem Ablösen getempert wurden, sehr deutlich den Einfluss des Substrats (und ganz besonders der Gitterfehlpassung) auf die sich einstellende Gitterstruktur beobachten.

\subsubsection{Hochenergetische Ionenbestrahlung}

Bei der Beschuss von polykristallinen $\mathrm{Fe}_{70} \mathrm{Pd}_{30}$-Filmen, die nach der Herstellung in der bct-Phase mit einer sehr feinen Mikrostruktur vorliegen [33, 102], mit 0,8 MeV Xe-Ionen erhält man Ergebnisse, die von denen der niederenergetischen Bestrahlung während der Deposition deutlich abweichen. Bei Raumtemperatur wird zwar ein deutliches Kornwachstum beobachtet, allerdings bleibt die Probe in der bct-Phase, während bei bei $400{ }^{\circ} \mathrm{C}$ die fcc-Phase gebildet wird. Dies deutet darauf hin, dass bei Raumtemperatur trotz der in der niederenergetischen Bestrahlung gefundenen sehr geringen Energieunterschiede zwischen den verschiedenen Gitterstrukturen keine ausreichende Triebkraft vorliegt, um die fcc-Struktur zu bilden. Simulationen mit SRIM zeigen, dass sich die Stoßkaskade auf ein Volumen von $150 \times 150 \times 200 \mathrm{~nm}^{3}$ erstreckt (siehe Abbildung 2.14). Im darauf folgenden Thermal Spike kann dieses Volumen als aufgeschmolzen betrachtet werden. Von den Rändern her 
thermalisiert der Thermal Spike wieder, indem die Wärme ins Volumen der Schicht abgeleitet wird. Hierbei erstarrt die Schmelze mit hoher Geschwindigkeit von den kristallinen Rändern aus. Bei den dabei herrschenden Temperaturen wäre eine Bildung der fcc-Struktur zu erwarten. Diese Schmelze ist jedoch von Körnern in der bct-Struktur umgeben, die als Kristallisationskeime wirken und in die Schmelze hinein wachsen. Dies erklärt, dass nur die Gitterstruktur beobachtet wird, die schon vor dem Ionenbeschuss vorlag.

Wird die Probe dagegen bei einer Temperatur von $400{ }^{\circ} \mathrm{C}$ bestrahlt, beobachtet man ein deutliches Wachstum der fcc-Phase. Um dieses Verhalten zu erklären, ist ein Zusammenspiel zweier Effekte notwendig: Zum einen wird die Schicht, wie auch in vielen anderen Experimenten gezeigt, zumindest teilweise entmischen, so dass sowohl bcc-Eisen als auch fcc $\mathrm{Fe}_{50} \mathrm{Pd}_{50}$-Körner vorliegen. Der Ionenstrahl sorgt nun dafür, dass in einem großen Volumen der Entmischung wieder entgegengewirkt wird, in dem sich in der ballistischen Stoßkaskade diese Bereiche wieder mischen. Beim Erstarren der Schmelze liegen nun als Kristallisationskeime nicht nur die bct-Körner vor, sondern auch die beim Entmischen gebildeten fcc $\mathrm{Fe}_{50} \mathrm{Pd}_{50}$-Körner, deren Wachstum offensichtlich bevorzugt abläuft, was bei der hohen Temperatur des Bereichs auch zu erwarten wäre. Bei der insgesamt auf die Probe gestrahlten Fluenz von $10^{16}$ Ionen $/ \mathrm{cm}^{2}$ ist davon auszugehen, dass das gesamte Probenvolumen mehrfach durch Thermal Spikes aufgeschmolzen war, und so im Lauf der Bestrahlung die gesamte Probe die fcc-Struktur annimmt.

\subsubsection{Nachträgliche Temperaturbehandlung}

Auch durch ein nachträgliches Tempern der Filme lässt sich die Gitterstruktur nach der Deposition noch verändern. Insbesondere die bei Raumtemperatur auf $\mathrm{SiO}_{2}$-Substrate aufgedampften Schichten lassen sich durch Tempern in die (bei $900{ }^{\circ} \mathrm{C}$ thermodynamisch stabile) fcc-Struktur überführen, die sich je nach Zusammensetzung dann beim Abkühlen in die fct-Struktur umwandelt. Dass hierbei neben den reinen Gleichgewichtsstrukturen auch das Vorhandensein passender Keime eine große Rolle spielt, zeigt sich sehr deutlich in den epitaktischen Schichten auf MgO. Das epitaktische Wachstum stabilisiert die fcc-Phase, die auch bei Raumtemperatur weiter erhalten bleibt. Wird der Film nun getempert, bildet das Substrat einen Kristallisationskeim, der alle anderen Keime einer möglichen Rekristallisation dominiert, so dass Filme, die auf dem Substrat auch für sehr lange Zeit bei sehr hohen Temperaturen getempert wurden, die wegen der Gitterfehlpassung gebildeten Defekte nicht ausheilen können. Die Oberfläche glättet sich hierbei weitestgehend, und verbleibende Stufen in der Oberfläche verlaufen parallel zu den (100)-Richtungen des Substrats, wie in Abbildung 5.18 zu erkennen ist. 
Die Experimente zu den unterschiedlichen Temperaturbehandlungen von $\mathrm{Fe}_{72} \mathrm{Pd}_{28}$, die in Abbildung 5.30 zusammengefasst sind, machen darüber hinaus den Einfluss der Defekte in der Schicht auf die Phasenbildung besonders deutlich. Da alle dort untersuchten Proben aus einer einzigen Schicht hergestellt wurden, die vor dem Ablösen zerteilt wurde, und alle Teile danach gemeinsam in einem Gefäß mit Natriumhydrogencarbonatlösung abgelöst wurden, kann hier ein Einfluss der chemischen Zusammensetzung ausgeschlossen werden. Alle getemperten Proben homogenisieren, so dass dass die wenigen Eisenausscheidungen, die nach dem langsamen Abkühlen nach der Deposition noch vorhanden waren, nun wieder im Volumen gelöst sind. Einzig durch verschiedene Temperaturbehandlungen lassen sich in diesen Proben die fcc, fct und die bcc-Struktur, sowie Mischungen dieser Phasen einstellen. In der Schicht, an der noch ein Rest des MgO-Substrats haftete, können die wegen der Gitterfehlpassung zum Substrat gebildeten Defekte nicht ausheilen, und ändert daher auch ihre Struktur nicht, auch die Lage des fcc(200)-Reflexes ist unverändert. In dem vollständig abgelösten Filmen dagegen nimt mit steigender Zeit und Temperatur die Zahl der Defekte immer weiter ab. Bei den verwendeten Temperaturen ist davon auszugehen, dass während des Temperns immer die fcc-Phase vorliegt (siehe Phasendiagramm in Abbildung 2.1), und sich eine Änderung der Struktur erst beim Abkühlen ergibt. In der am wenigsten relaxierten Probe $\left(15\right.$ Minuten bei $900^{\circ} \mathrm{C}$ ) zeigt sich, dass sich der fcc(200)-Reflex etwas zu größeren Winkeln verschoben hat (also entgegen der Richtung, die die Fehlpassung zum Substrat erzwingen würde), und außerdem eine Schulter aufweist, die den Beginn der Bildung der fct-Phase zeigt. Wird die Probe dagegen stattdessen für die selbe Zeit bei $1000^{\circ} \mathrm{C}$ getempert, ist der Reflex der fcc-Phase vollständig verschwunden, und stattdessen liegt der größte Teil der Probe in der fct-Struktur vor. Darüber hinaus ist auch ein kleiner Peak der bcc-Struktur zu erkennen. Wird eine Probe bei dieser Temperatur für 20 Stunden getempert, so ist der fct-Peak ebenfalls vollständig verschwunden, und die gesamte Probe liegt in der bcc-Struktur vor, die bei dieser Zusammensetzung auch in polykristallinen Folien nach dem Tempern beobachtet wird [131].

Zusammenfassend lässt sich somit sagen, je mehr die Proben während des Tempern ohne den Einfluss des Substrats relaxieren konnten, desto weiter können sie sich beim Abkühlen entlang des Bain-Pfades umwandeln. Dies lässt umgekehrt auch den Schluss zu, dass die verschiedenen Zwischenschritte zwischen der fcc- und der bcc-Struktur maßgeblich durch die jeweils vorhandene Art und Anzahl der Defekte bestimmt werden und damit die Energieunterschiede zwischen den Strukturen recht gering sind. Dies bestätigt die Ergebnisse der quantenmechanischen Rechnungen, die übereinstimmend einen sehr flachen Verlauf der Energie entlang des Bain-Pfades ergeben, wobei in 
einer unrelaxierten Simulationszelle die fcc-Struktur als Grundzustand gefunden wird [13], während in einer relaxierten Simulationszelle eine schwach verzerrte bct-Struktur die niedrigste Energie aufweist [15]. Im Legierungssystem Eisen-Palladium existiert keine langreichweitig geordnete $\mathrm{L}_{2}$ Phase bei der Zusammensetzung $\mathrm{Fe}_{75} \mathrm{Pd}_{25}$, wie sie im System Eisen-Platin auftritt. Auch wurden keine Überstruktur-Peaks gefunden, die auf solch eine Fernordnung hinweisen würden. Es ist jedoch möglich, dass trotzdem eine gewisse Tendenz hin zu einer Nahordnung existiert. Es wäre vorstellbar, dass sich der Grad der Nahordnung durch das Tempern des abgelösten Films ohne die Randbedingungen des Substrats ebenfalls deutlich verändert.

\subsection{Elastische Eigenschaften}

Wie in Kapitel 2.1.3 erläutert, ist in der Nähe des Martensitübergangs ein Erweichen des Gitters und eine Instabilität gegenüber Verformungen zu erwarten. An dünnen Schichten, insbesondere an solchen, die auf einem Substrat haften, sind die elastischen Eigenschaften nur in lokalen Messungen zugänglich, wie sie beispielsweise mittels Nanoindentation möglich sind.

\subsubsection{Polykristalline Schichten}

Vergleicht man die aufgenommenen Kraft-Eindringtiefen Kurven für polykristalline Schichten auf $\mathrm{SiO}_{2}$ (Abbildungen 5.33 und 5.34) mit denen, die an einer abgelösten einkristallinen Schicht aufgenommen wurde (Abbildung 5.28), fällt zunächst die starke Streuung der Messungen an den polykristallinen Schichten auf. Bei diesen Messungen an den polykristallinen Filmen wurde jeweils an einem Punkt der Probe die Kraft bis zu einem vorgegebenen Maximalwert erhöht, und der E-Modul aus der Steigung der Entladekurve und der Fläche der zurückbleibenden plastischen Verformung bestimmt. Wie an den beiden in Abbildung 6.5 gezeigten Indents zu erkennen ist, kann diese Bestimmung der Kontaktfläche mit einem sehr großen Fehler behaftet sein, insbesondere wenn der Ort des Indents ungünstig (z. B. nahe an einer tief einschneidenden Korngrenze) auf der rauen Oberfläche (siehe Abbildung 5.35) der Schicht liegt.

Die in Abbildung 6.6 dargestellten E-Moduli der polykristallinen Proben, deren Mittelwert um $120 \mathrm{GPa}$ liegt, unterscheiden sich kaum, obwohl nur eine der beiden Proben martensitisch ist, während die andere im Austenit vorliegt. Dies deutet darauf hin, dass mit diesen Experimenten keine Bewegung der Zwillingsgrenzen erreicht werden konnte. Auch die Ergebnisse der Röntgenbeugung an diesen Proben deuten darauf hin, dass die Probe 


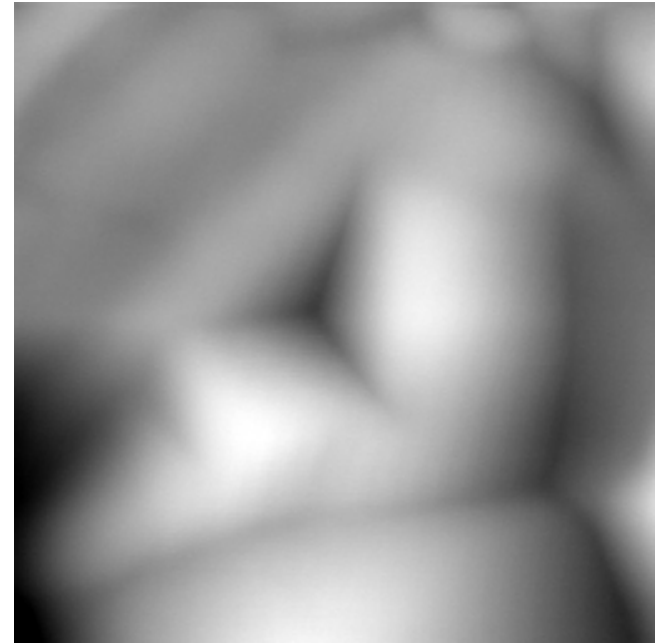

(a) Deutlicher Indent

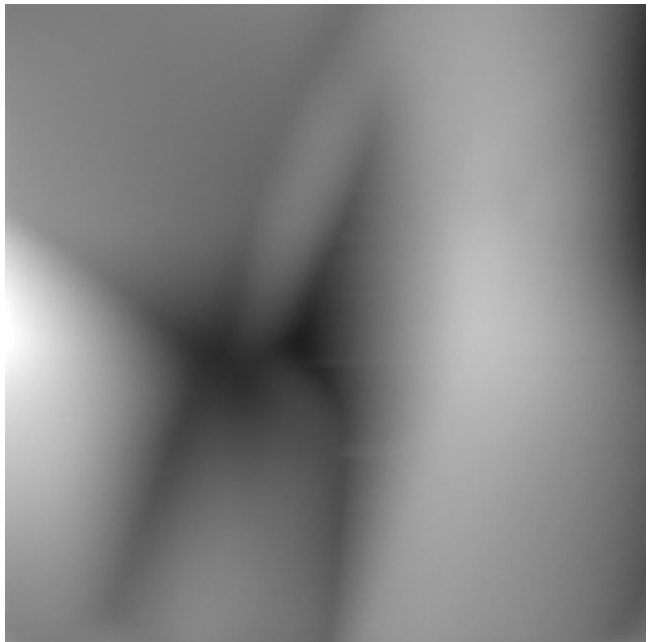

(b) Undeutlicher Indent

Abbildung 6.5: AFM-Aunahmen der Indents an den polykristallinen Schichten, Kantenlänge $1 \mu \mathrm{m}$, Höhenskala $100 \mathrm{~nm}$.

eine sehr feine Zwillingsstruktur aufweist. In Abbildung 5.32 ist unterhalb des Martensitübergangs weder ein (200) noch ein (002)-Peak zu erkennen, während der (111)-Reflex fast unverändert bleibt. Solch eine feine Verzwillingung wäre dann zu erwarten, wenn die Umwandlung nicht lawinenartig durch die ganze Probe läuft, sondern an vielen Stellen beginnt, aber in ihrer Ausbreitung immer wieder gehemmt wird. Dabei stellt sich eine sehr feingliedrige Zwillingsstruktur ein, ähnlich wie beim Erstarren eines Eutektikums, das ebenfalls gleichzeitig an vielen Stellen beginnt, und zu einer besonders feinen Mikrostruktur führt. Solch eine durch eine hohe Defektdichte und möglicherweise auch das Oberflächenrelief verursachte geringe Beweglichkeit der Zwillingsgrenzen kann somit erklären, dass der zusätzliche Freiheitsgrad der Umorientierung der Martensitvarianten bei mechanischer Belastung nicht aktiviert werden kann.

\subsubsection{Abgelöste freistehende Schichten}

Die Messung der elastischen Eigenschaften der abgelösten einkristallinen Schicht wurde im CSM-Modus durchgeführt, bei dem schon während des Ladevorgangs in sehr kurzen Abständen die Kraft verringert wird, um so die elastische Antwort der Probe zu bestimmen, so dass hier in einer Messung viele Werte für den E-Modul bestimmt werden können, und dessen Eindringtiefen-Abhängigkeit untersucht werden kann. 


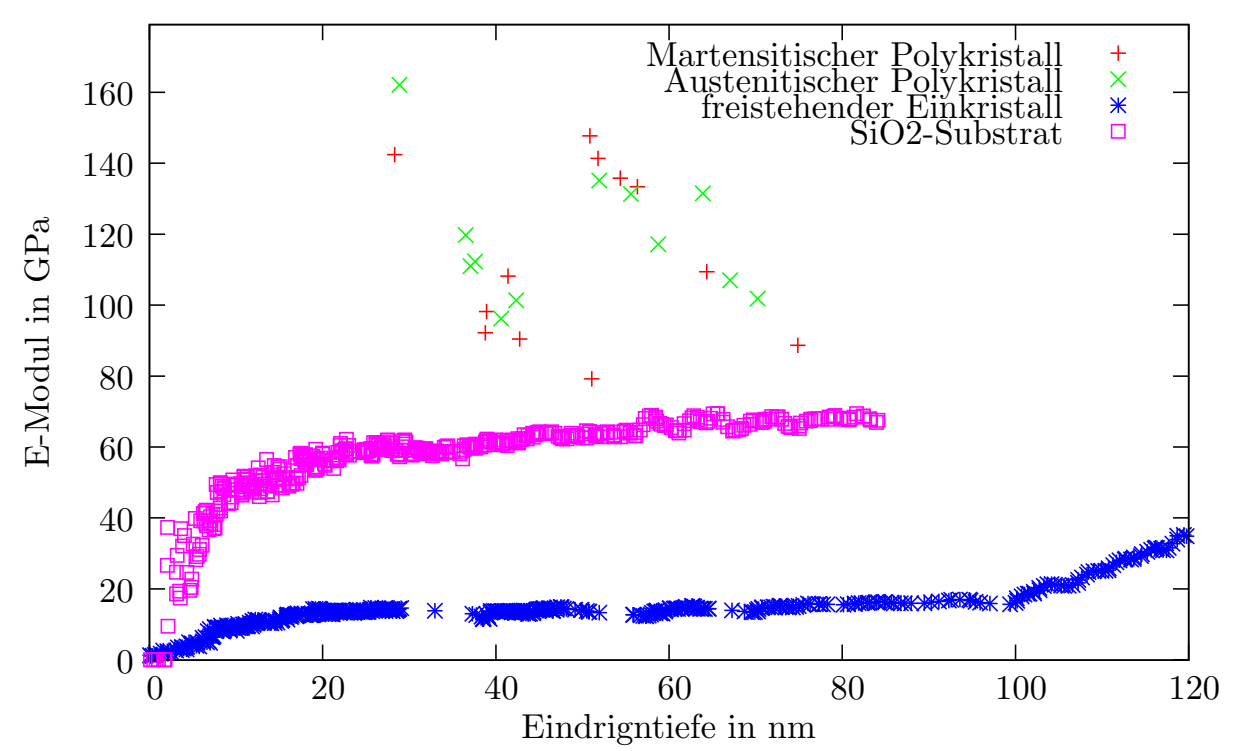

Abbildung 6.6: Vergleich des E-Moduls zweier polykristallinen Filme auf $\mathrm{SiO}_{2}$ (einer martensitisch, einer austenitisch), eines abgelösten einkristallinen Fils, der auf einem $\mathrm{SiO}_{2}$-Substrat aufliegt und eines leeren Substrats.

Der elastische Modul der abgelösten einkristallinen Schicht lässt sich in Abbildung 6.6 in einem Tiefenbereich von 20 bis $100 \mathrm{~nm}$ zu etwa $14 \mathrm{GPa}$ bestimmen. Ab einer Eindringtiefe von etwa $100 \mathrm{~nm}$ steigt der elastische Modul deutlich an, und nähert sich deutlich dem Wert des Substrats. Es gibt insgesamt drei Bereiche, in denen die Spitze sehr weit in die Probe eindringt, und folglich der berechnete Modul deutlich einbricht (in Abbildung 5.28 deutlich zu erkennen). Solche Ereignisse werden als Pop-in bezeichnet, und können sowohl von einer spannungsinduzierten Martensitumwandlung (also Superelastizität) herrühren, aber auch durch Reißen des Materials oder durch schlechte Adhäsion zum Substrat verursacht werden. Nach dem Entladen war hierfür kein eindeutiger Beweis zu finden, da die Schicht in der Umgebung des Indents beim tieferen Eindringen zerstört wurde. Andererseits lässt sich ein spannungsinduzierter Martensitübergang auch nicht ausschließen, da ja beim Entlasten wieder der umgekehrte Übergang zum Austenit stattfinden würde, somit auch keine Spuren zurückblieben. Unabhängig vom mikroskopischen Mechanismus der Pop-ins bleib festzuhalten, dass das Kristallgitter sehr weich ist, was darauf hindeutet, dass die Schicht sich bei Raumtemperatur schon sehr in der Nähe des Martensitübergangs befindet. Auch für freistehende $\mathrm{Fe}_{70} \mathrm{Pd}_{30}$-Schichten, die vor kurzem mittels Sputtern auf eine Opferschicht aus Gold von Bechthold et al. hergestellt wurden, wurde in 
Zugversuchen ein E-Modul von 15 GPa gefunden. Diese Schichten wachsen beim Deponieren direkt in der bct-Phase auf, und zeigen auch bei Temperaturänderungen keinen strukturellen Übergang. Im Gegensatz zu den Zugversuchen wird in diesen Proben mittels Nanoindentation ein sehr viel höherer E-Modul von 140 GPa gefunden. Dieser überraschende Unterschied wird von Bechthold et al. damit erklärt, dass das komplexe dreidimensionale Spannungsfeld an der Spitze des Nanoindenters im Gegensatz zum uniaxialen Zug in der bct-Stuktur keine günstige Reorientierung auslösen könne [132].

\subsection{Strukturelle Übergänge}

Wie in Kapitel 5.5 dargestellt, kann in polykristallinen Proben auf einem $\mathrm{SiO}_{2}$-Substrat eine martensitische Umwandlungstemperatur von $60^{\circ} \mathrm{C}$ beobachtet werden. Während in den epitaktischen Schichten auf $\mathrm{MgO}$ der Einfluss des Substrats einen Martensitübergang verhindert, zeigen freistehende getemperte Proben einen deutlichen Übergang, bei dem die bcc bzw. bctStruktur entsteht, und der entgegen den Beobachtungen an Bulkproben reversibel ist.

\subsubsection{Polykristalline Schichten}

In den polykristallinen Filmen beobachtet man bei einer Zusammensetzung von $\mathrm{Fe}_{72} \mathrm{Pd}_{28}$ in den temperaturabhängigen Röntgenmessungen, dass oberhalb von $60^{\circ} \mathrm{C}$ der fcc(200)-Peak klar zu erkennen ist, während bei tieferen Temperaturen dieser Peak verschwindet. In dieser Probe sind entgegen der Erwartungen keine fct(200) bzw. fcc(002)-Reflexe zu beobachten. Diese lassen sich auch durch ein systematisches Verkippen der Probe gegenüber der Reflexionsebene (Rocking Curve) nicht einstellen, so dass eine Verkippung der Ebenen als Ursache ausgeschlossen werden kann. Der (111)-Reflex ist über den gesamten Temperaturbereich unverändert, somit kann ausgeschlossen werden, dass sich die Probe aus dem Fokus der Röntgenröhre bewegt hat oder gekippt ist, oder das die Temperaturänderung z.B. durch Kondensation die Röntgenmessung negativ beeinflusst. Vielmehr bedeutet dies, dass sich während der Transformation die Länge der Raumdiagonalen der Gitterzelle nicht oder nur minimal ändert, so dass auch über verschiedene Zwillingsvarianten hinweg eine kohärente Streuung an den $\{111\}$-Ebenen möglich ist, während entlang der $\langle 200\rangle$-Richtungen durch die Verzwillingung die Kohärenzlänge so kurz wird, dass der Peak so weit verbreitert und abgeflacht ist, dass er nicht mehr vom Hintergrund zu trennen ist, wie es die Scherrer-Formel für sehr kleine Strukturen vorhersagt [115]. 


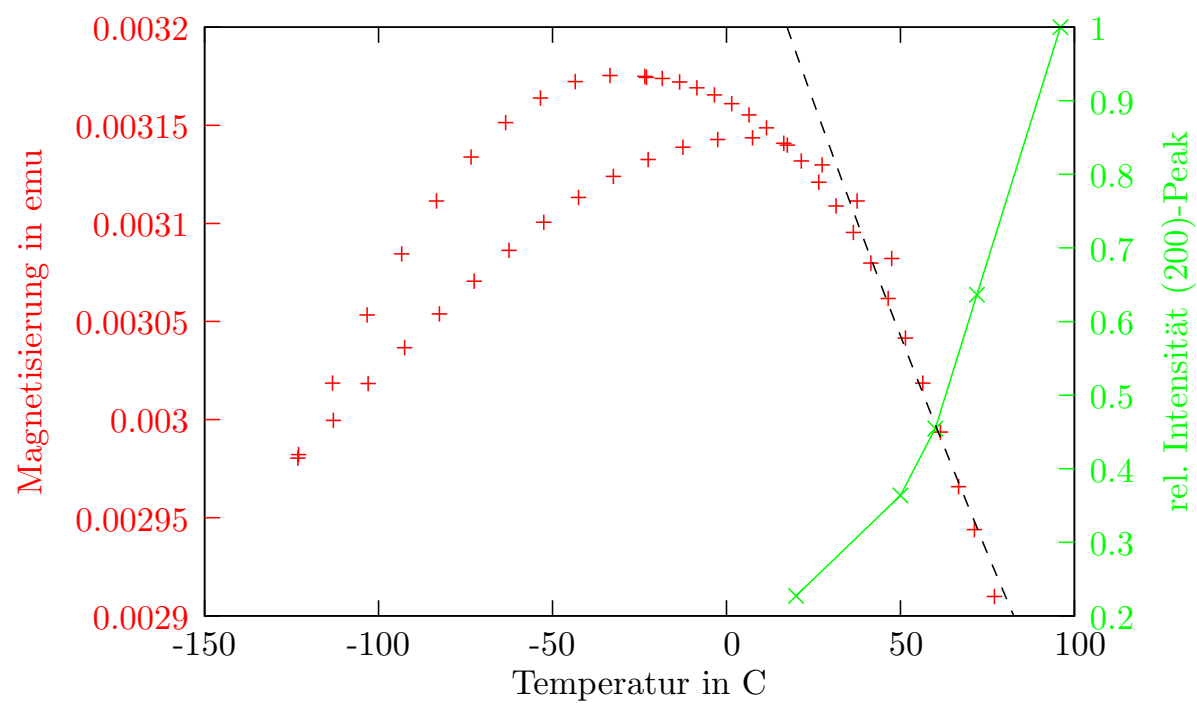

Abbildung 6.7: Vergleich der Änderung der Gitterstruktur und der Magnetisierung in einem äußerem Feld von $30 \mathrm{mT}$ an einer polykristallinen $\mathrm{Fe}_{72} \mathrm{Pd}_{28^{-}}$ Schicht

Außer dem bereits in Abbildung 5.31 dargestellten elektrischen Widerstand zeigten auch die magnetischen Eigenschaften dieser Probe eine deutliche Signatur des Martensitübergangs. Abbildung 6.7 zeigt die Magnetisierung einer solchen transformierenden Probe in einem konstanten externen Feld von $30 \mathrm{mT}$. Als Maß für die strukturelle Umwandlung ist in dieser Abbildung zusätzlich die relative Intensität des fcc(200)-Reflexes dargestellt, der bei dieser Probe oberhalb von $60^{\circ} \mathrm{C}$ deutlich ausgeprägt ist, darunter aber verschwindet (siehe Abbildung 5.32).

Für hohe Temperaturen nimmt die Magnetisierung etwa linear mit fallender Temperatur zu, wie man es in diesem Bereich für einen Ferromagneten auch erwarten würde. Für Temperaturen unterhalb des Martensitübergangs jedoch wird die Kurve zunächst immer flacher, und fällt für tiefe Temperaturen sogar deutlich. Außerdem öffnet sich hier eine deutliche Hysterese; in diesem Bereich ist die Magnetisierung beim anschließenden Aufheizen der Probe deutlich geringer als beim Abkühlen. Dieser Effekt ist in großen Magnetfeldern so nicht zu beobachten, daher ist davon auszugehen, dass es sich hierbei im Wesentlichen nicht um eine Änderung der Magnetischen Momente pro Einheitszelle handelt, sondern sich vielmehr die magnetische Anisotropie der Schicht beim Martensitübergang ändert. Das selbe Verhalten wird auch beim Martensitübergang von Bulk-Einkristallen beobachtet, die in einem Magnetfeld von $50 \mathrm{mT}$ abgekühlt wurden [133]. Auch andere Untersuchungen zeigen, 
dass die magnetische Anisotropie beim Übergang zwischen der fcc- und der fct-Struktur deutlich zunimmt, aber auch in der martensitischen Phase mit fallender Temperatur weiter ansteigt $[134,32]$. Dieses Verhalten ähnelt auch dem Verlauf der tetragonalen Verzerrung in $\mathrm{Fe}_{70} \mathrm{Pd}_{30}$ [39]. Auch diese ändert sich im Gegensatz zu NiMnGa nicht sprunghaft bei der Phasenumwandlung, sondern folgt bis zu tiefen Temperaturen einem kontinuierlichen Verlauf, weshalb der Übergang als ein Übergang fast schon zweiter Ordnung bezeichnet wird [135, 136, 22, 39]. Dies erklärt, warum sich der Martensitübergang nicht als Sprung in den Magnetisierungsmessungen abzeichnet, sondern eine kontinuierliche Änderung über einen großen Temperaturbereich bewirkt.

Der Abfall der Magnetisierung in Abbildung 6.7 kann also dadurch erklärt werden, dass durch das externe Feld im Austenit ein größerer Anteil der magnetischen Momente in Feldrichtung ausgerichtet wurde, und nach dem Übergang ein Teil der Magnetisierung durch die Anisotropie des Martensits aus der Richtung des externen Felds hinausgedreht wird. So lässt sich auch die beobachtete Hysterese verstehen: Beim Aufwärmen nimmt die Anisotropie ab, und mehr Domänen richten sich wieder in dem äußeren Feld aus. Durch die in der Schicht vorhandenen Defekte, insbesondere die Korngrenzen, sind diese Domänen verschieden stark gepinnt, so dass sie erst bei einer verschieden großen Änderung der Anisotropie zurückgedreht werden. Oberhalb der Martensittemperatur erhält man wieder das selbe lineare Verhalten wie zuvor.

\subsubsection{Reversibler fcc-bcc-Übergang in getemperten ab- gelösten Proben}

Während die polykristallinen Proben einen Martensitübergang von der fcc in die fct-Struktur zeigen, kann in freistehenden einkristallinen Proben ein ungewöhnlicheres Verhalten beobachtet werden. In einer $\mathrm{Fe}_{70} \mathrm{Pd}_{30}$-Schicht, von der mit Natriumhydorgencarbonat das Substrat langsam und schonend abgelöst wurde, und die danach für 30 Minuten bei $900{ }^{\circ} \mathrm{C}$ getempert wurde, ist bei tiefen Temperaturen ein (110)-Reflex der raumzentrierten Struktur zu beobachten. In diesem Abschnitt soll nun diskutiert werden, warum dieser Übergang auftritt, weshalb der Übergang von der fcc zur bct-Struktur in der Literatur als irreversibel bezeichnet wird, und warum hier trotzdem ein reversibler Übergang beobachtet wird.

Den in Abbildung 5.38(b) bei $-100{ }^{\circ} \mathrm{C}$ gefundenen $\{110\}$-Reflexen kann nicht exakt der selbe Ebenenabstand zugeordnet werden. Allerdings ist auch eine Ungenauigkeit der Messung (insbesonders durch die Einbettung der Probe in das Wachs und die damit verbundene ungenaue Justage) nicht 
ausgeschlossen. Insofern erlauben die Messergebnisse keine eindeutige Entscheidung, ob es sich nun um eine exakt kubische bcc-Struktur, oder eine schwach tetragonal verzerrte bct-Struktur handelt. Es lässt sich daraus jedoch als Obergrenze ein Achsenverhältnis von $c / a=1,03$ abschätzen. Dies steht in sehr guter Übereinstimung mit den Vorhersagen von Gruner, der eine schwach verzerrte bct-Struktur als Grundzustand findet (siehe Abbildung 2.4 rechts ) [15]. Dieser bct-Grundzustand ergibt sich in den quantenmechanischen Rechnungen allerdings nur, wenn den Atomen erlaubt wird, ihre Positionen zu relaxieren, und tritt in einem starren Gitter nicht auf. In diesem Fall finden verschiedene Rechnungen ein Energieminimum bei der fcc bzw. fct-Struktur [15, 13]. Dass die genaue Mikrostruktur einen entscheidenden Einfluss darauf hat, ob ein Übergang in eine Struktur mit geringerer Energie auch tatsächlich abläuft, zeigt sich auch in Molekulardynamik-Simulationen zum fcc nach bcc Übergang von reinem Eisen [137]. Hier stellt man ebenfalls fest, dass die Randbedingungen einen großen Einfluss auf das Auftreten der strukturellen Umwandlung haben. So wurde dort die Umwandlung nur dann beobachtet, wenn entweder die gesamte Simulationszelle mit periodischen Randbedingungen spannungsfrei gehalten wurde (NTp-Ensembe mit variablem Volumen), oder in dünnen Filmen, die eine Korngrenze enthalten, während in Filme ohne Korngrenze die Umwandlung nicht auftritt [137].

Es ist auch aus Experimenten bekannt, dass außer der Zusammensetzung auch die Mikrostruktur einen großen Einfluss auf das Umwandlungsverhalten von Eisen-Palladium-Proben hat. In $\mathrm{Fe}_{69.2} \mathrm{Pd}_{30.8}$-Nanopartikeln mit einer mittleren Größe um $25 \mathrm{~nm}$ wurde gar kein Martensitübergang beobachtet [138], in polykristallinen $\mathrm{Fe}_{69.9} \mathrm{Pd}_{30.1}$-Proben mit einer Korngröße von etwa $2.6 \mu \mathrm{m}$ liegt die Übergangstemperatur etwa 100 Grad tiefer als in Volumenproben, bei einer Korngröße von etwa $10 \mu \mathrm{m}$ nur noch 40 Grad tiefer als in Volumenproben, deren Übergangstemperatur von $18^{\circ} \mathrm{C}$ bei einer Korngröße von $266 \mu \mathrm{m}$ erreicht wird [138].

In dieser Hinsicht ist die Situation eines freistehenden einkristallinen Films einzigartig, da er einerseits in der Schichtebene Dimensionen im Millimeterbereich aufweist, die Bulk-Eigenschaften vermuten lassen, aber andererseits in die Richtung senkrecht zur Schichtebene die Korngröße durch die Schichtdicke im Bereich von deutlich unter einem Mikrometer begrenzt wird. Wie auch beim Invar-Effekt in Abbildung 5.36 sehr deutlich zu sehen, übt außer den verschiedenen auftretenden Längenskalen das Substrat einen großen Einfluss auf die Eigenschaften des Films aus, so dass die intrinsischen Eigenschaften eines Films immer mit denen des Substrats gekoppelt sind.

Außer durch sein großes Seitenverhältnis zeichnet sich ein freistehender Film, der nur lose auf einem $\mathrm{SiO}_{2}$-Substrat aufliegt, auch dadurch aus, dass er sich unter auftretenden Spannungen verbiegen und seine Länge ändern 
kann, so dass auch große Spannungen abgebaut werden können. Daher endet hier die Martensitumwandlung nicht in der fct-Struktur, sondern die Transformation erstreckt sich über große Teile des Bain-Pfads bis zur bcc bzw. bct-Struktur. Es ist daher davon auszugehen, dass in einem transformierenden Bereich, der sich über die gesamte Filmdicke erstreckt, die Spannungen abgebaut sind, und nur an der Grenzfläche zu einem noch nicht transformierten Bereich Spannungen auftreten. Es ist vorstellbar, dass diese Grenzfläche sich nicht durch die ganze Probe bewegt, sondern dass sie an Defekten gepinnt wird, und sich so die Koexistenz von flächenzentrierter und raumzentrierte Phase in Abbildung 5.37 verstehen lässt. In diesem Fall ist davon auszugehen, dass sich in der Umgebung dieser Grenzfläche spannungsinduziert die fct-Struktur ausbildet, was auch das schwache Auftreten nur eines der fct(200)-Reflexe erklärt. Solche Pinningzentren können zum Beispiel auch nach dem Tempern für 30 Minuten bei $900^{\circ} \mathrm{C}$ noch vorhandene Versetzungen sein. Dass zu diesem Zeitpunkt noch nicht alle Defekte ausgeheilt sind ist wahrscheinlich, da sich in den untersuchten $\mathrm{Fe}_{72} \mathrm{Pd}_{28}$-Schichten zeigt, dass sich die Struktur der Probe weiter verändert, wenn sie statt bei $900^{\circ} \mathrm{C}$ für 20 Stunden bei $1000^{\circ} \mathrm{C}$ getempert werden.

Sowohl die Magnetisierungsmessungen als auch die Röntgenmessungen zeigen, dass es sich bei der Strukturänderung der getemperten freistehenden $\mathrm{Fe}_{70} \mathrm{Pd}_{30}$-Probe um einen reversiblen Vorgang handelt. Dies ist in sofern ungewöhnlich, als dass der Übergang der fcc oder fct in die bct oder bcc-Struktur in Volumenproben als nicht thermoelastisch und irreversibel bezeichnet wird [136]. Von Oshima et al. wird die Umwandlung der fcc- in die bct-Struktur einer $40 \mu \mathrm{m}$ dicken polykristallinen $\mathrm{Fe}_{70,3} \mathrm{Pd}_{29,7}$-Folie so beschrieben, dass trotz der sehr kleinen Volumenänderung von $0,2 \%$ bei der thermoelastischen Umwandlung zwischen der fcc und der fct-Phase eine gewisse elastische Energie in der austenitischen Matrix gespeichert wird, und diese zu einer Verzwillingung im Martensit führt [135, 22]. Die hiermit verbundene Grenzflächenenergie nimmt mit zunehmender Zwillingsdichte ebenfalls weiter zu, so dass die Gesamtenergie wieder zunimmt. Dadurch kann die fct-Struktur instabil werden, und sich schlagartig in die bct-Struktur umwandelt [135].

Durch den von Oshima [135] beschriebenen Mechanismus wird klar, dass die Umwandlung von der bcc- zur fcc-Struktur sehr schwierig ist, da die zu überwindende Aktivierungsenergie-Barriere zur bct-Seite hin sehr steil abfällt, da sich als Zwischenzustand eine fct-Phase mit sehr hoher Zwillingsdichte bilden müsste, die dann bei weiterem Fortschreiten der Umwandlung in Richtung einer fcc-Struktur die Zahl dieser Zwillingsgrenzen langsam abbauen würde. Für eine Rückumwandlung in die fct-Struktur müsste gleichzeitig die gesamte Grenzflächenenergie all dieser Zwillingsgrenzen aufgebracht 
werden,so dass eine große Überhitzung notwendig wäre, um den Übergang tatsächlich ablaufen zu lasen [40]. So beginnt eine Probe zu entmischen, bevor eine hinreichend hohe Temperatur erreicht ist.

Darüber hinaus ist es wie auch beim thermischen Formgedächtniseffekt im Martensit sehr leicht, durch eine geeignete Anordnung der langen und kurzen Achsen den Randbedingungen an einen transformierenden Bereich gerecht zu werden. Dieser Freiheitsgrad existiert bei der Rücktransformation nicht, und macht die Rücktransformation in eine kubische Struktur dementsprechend schwieriger. Eine freistehende einkristalline Schicht kann allerdings wie bereits diskutiert, diese Verspannungen sehr leicht durch Verformung abbauen. Außerdem existieren noch Bereiche der Probe, die auch bei niedrigen Temperaturen in der fcc-Struktur vorliegen, so dass im Gegensatz zu den von Bechthold et al. hergestellten Schichten [132] bereits Keime der fcc-Struktur existieren, und die Phasengrenze sich nur wieder durch die Probe bewegen muss.

In den Magnetisierungsmessungen in den Abbildungen 5.40 und 5.39 ist außer der schon in den polykristallinen Proben diskutierten Änderung der magnetischen Anisotropie auch ein irreversibler Prozess zu beobachten, wenn die Probe zum ersten Mal über Raumtemperatur erwärmt wird. Dieser hat nichts mit dem Martensitübergang zu tun, sondern ist auf eine Relaxation der Probe bei dieser Temperaturänderung zurückzuführen: Während des Temperns ist eine erhöhte Zahl von Leerstellen zu erwarten, deren Gleichgewichtskonzentration in Abhängigkeit der Temperatur durch den damit verbundenen Entropiegewinn bestimmt wird. Werden die Proben dann in Wasser abgeschreckt, sind zwar die Versetzungen reduziert, allerdings wird ein Teil der Gleichgewichtsleerstellen nicht schnell genug ausheilen können, so dass die Filme immer noch eine erhöhte Defektkonzentration aufweisen. Diese Leerstellen können nun zumindest teilweise an die Oberfläche wandern und dort anihilieren, wenn die Filme moderat erwärmt werden. Eine solche Relaxation der Struktur und eine damit verbundene Zunahme der Magnetisierung wurde auch beim Tempern bei Temperaturen von unter $220^{\circ} \mathrm{C}$ in gesputterten $\mathrm{Fe}_{75} \mathrm{Pd}_{25}$-Proben beobachtet [139], und erklärt die Änderung der Magnetisierung zwischen dem ersten und den folgenden Temperaturzyklen in Abbildung 5.40 und 5.39. 


\section{Kapitel 7}

\section{Zusammenfassung und Ausblick}

Im Rahmen dieser Arbeit wurde der Einfluss verschiedener Depositionsverfahren und Substrate auf die Struktur und die intrinsischen Eigenschaften dünner Eisen-Palladium-Filme untersucht.

Beim Aufdampfen polykristalliner Filme bei erhöhten Substrattemperaturen zeigt sich, dass sich durch die geringe Neigung zur Benetzung von $\mathrm{SiO}_{2}$ mit FePd raue, entmischte Filme bilden. Dagegen können mittels Ionenstrahlunterstützter Deposition je nach Zusammensetzung bei Raumtemperatur sehr glatte Schichten in der fcc oder der bcc-Struktur hergestellt werden. In einem Bereich zwischen 28 und 33\% Palladium erhält man Schichten, in denen Körner beider Kristallstrukturen vorhanden sind. Durch die Ionenbestrahlung werden hier statt weniger großer Keime (wie beim Aufdampfen bei hohen Substrattemperaturen) viele kleine Keime gebildet, da durch ballistische Stöße Metallatome aus schon vorhandenen Keimen entfernt und auf dem Substrat redeponiert werden. In diesen kleinen Keimen ist sowohl die fcc als auch die bcc-Struktur stabil, und weitere deponierte Atome passen sich der jeweils lokal vorhandenen Gitterstruktur an. Durch den Materialtransport von den Hügelflanken in die Täler der Struktur entstehen so durch die Ionenbestrahlung sehr glatte Filme, die eine feinkörnige Struktur aufweisen.

In getemperten polykristallinen Filmen wird in Röntgenbeugungsexperimenten, in Messungen der Magnetisierung und des elektrischen Widerstands ein Martensitübergang beobachtet, der je nach Zusammensetzung bei Temperaturen von bis zu $60^{\circ} \mathrm{C}$ abläuft. Hierbei stellt sich jedoch durch das Substrat und die defektreiche Mikrostruktur eine sehr hohe Dichte an Zwillingsgrenzen ein. In diesen Polykristallen kann keine Änderung der elastischen Eigenschaften zwischen martensitischen und austenitischen Proben beobachtet werden, was darauf hindeutet, dass die Zwillingsgrenzen nicht beweglich sind, da sie von der defektreichen Mikrostruktur gepinnt sind.

Bei in mittels gepulster Laserdeposition bei Raumtemperatur epitaktisch 
auf $\mathrm{MgO}(100)$ deponierten Filmen zeigt sich, dass die Misfit-Spannungen zu einer massiven Verzwillingung und einem damit verbundenen Zusammenbruch der Epitaxie nach Schichtdicken von 10 bis $20 \mathrm{~nm}$ führen.

Im Gegensatz dazu lassen sich mittels Elektronenstrahlverdampfen bei Substrattemperaturen von $690^{\circ} \mathrm{C}$ und darüber dicke, spannungsfreie, epitaktische Filme in der fcc-Struktur herstellen. Aufgedampfte Schichten relaxieren die Gitterfehlpassung bei Substrattemperaturen unter $690^{\circ} \mathrm{C}$ teilweise mittels Partialversetzungen, deren Wechselwirkung mit den sich unter diesen Depositionsbedingungen ebenfalls bildenden Eisenausscheidungen in MD-Simulationen untersucht wurden. Hierbei ist deutlich zu erkennen, dass die Grenzflächen zwischen der fcc-Matrix und den bcc-Ausscheidungen die Bewegung von Zwillingsgrenzen behindert, und daher in den Filmen mit Ausscheidungen höhere Spannungen auftreten.

In den bei Temperaturen über $690^{\circ} \mathrm{C}$ hergestellten Filmen werden die Spannungen über volle Versetzungen abgebaut. Diese Versetzungen, die auch bei Raumtemperatur noch in den Proben vorhanden sind, verhindern allerdings einen Martensitübergang in diesen Filmen. Werden die Filme jedoch vom Substrat abgelöst, und die Defekte durch eine weitere Temperaturbehandlung reduziert, erhält man in $\mathrm{Fe}_{72} \mathrm{Pd}_{28}$ je nach Temperaturbehandlung statt der fcc-Phase auch die fct- oder die bcc-Phase bei Raumtemperatur.

Nach dem Ablösen ebenfalls getemperte $\mathrm{Fe}_{70} \mathrm{Pd}_{30}$-Proben zeigen einen reversiblen Übergang zwischen der fcc- und einer (möglicherweise schwach tetragonal verzerrten) raumzentrierten Struktur. Da solch ein freistehender einkristalliner Film sehr leicht Spannungen durch Verformung und Dehnung abbauen kann, kann sich hier die Gitterstruktur den ganzen Bain-Pfad entlang umwandeln. Bei einer Temperatur von $-145^{\circ} \mathrm{C}$ koexistieren die fcc, die bcc/bct- und ein geringer Teil der fct-Struktur miteinander. Dies ist vermutlich dadurch zu erklären, dass die Grenzfläche der fcc-bcc-Umwandlung nicht durch die ganze Probe propagieren kann, sondern an Defekten gepinnt wird. Dies liefert auch eine Erklärung, warum diese Umwandlung beim Erwärmen wieder rückwärts abläuft, so dass die Probe nach dem Erwärmen auf Raumtemperatur wieder vollständig in der fcc-Phase vorliegt. Die Verzerrungen, die bei dieser Rücktransformation auftreten, können von dem freistehenden Film und den Oberflächen leicht kompensiert werden, während dies in Polykristallen und Bulkproben nicht möglich ist, und die Transformation dort irreversibel ist.

In freistehenden einkristallinen austenitischen Filmen findet man einen sehr geringen Elastizitätsmodul von etwa $14 \mathrm{GPa}$, der zeigt, dass sich die Instabilität des austenitischen Gitters schon deutlich vor dem Martensitübergang abzeichnet, und auch vorhanden ist, wenn wegen der Versetzungen in der Schicht keine Martensitbildung beim Kühlen beobachtet wird. 
Diese Ergebnisse bestätigen die quantenmechanische Vorhersagen über einen sehr flachen Verlauf der potentiellen Energie entlang des Bain-Pfades [15, 13, 14], da es mit einem geringen Energieaufwand möglich ist, das Gitter zu dehnen. Wenn der Struktur die Gelegenheit gegeben wird, Defekte und Verspannungen abzubauen, stellt sich bei tiefen Temperaturen eine höchstens schwach verzerrte bcc-Struktur ein, wie sie auch in quantenmechanischen Rechnungen, in denen alle Atome an die Idealpositionen relaxiert sind, gefunden wird [15].

Für zukünftige, über diese Arbeit hinausgehende Untersuchungen bietet das Zulegieren einer dritten Komponente vielfältige Möglichkeiten. Es existieren verschiedene Vorschläge wie Platin, Cobalt, Nickel, Kupfer, Mangan, Aluminium oder Bor, die teils in Bulkproben oder polykristallinen Schichten bereits hergestellt wurden, teils auch theoretisch vorhergesagt wurden [140, 141, 142, 143, 144, 145, 146]. Um die für Anwendungen gewünschte fct-Phase auch bei tieferen Temperaturen gegen eine weitere Umwandlung in die bct und bcc-Phase zu stabilisieren, stellt möglicherweise auch in dünnen Schichten das Zulegieren von Mangan eine Option dar. In Bulk-Polykristallen lässt sich durch die Substitution von 2,5 Prozent Eisen durch Mangan der Übergang in die bct-Phase unterdrücken und gleichzeitig die Transformationstemperatur der fct-Phase erhöhen [145]. Außerdem existieren Hinweise darauf, dass sich die magnetische Anisotropie, und damit die maximal erreichbare Spannung eines Aktors, durch die Zugabe von wenigen Prozent Platin deutlich erhöhen lässt [146].

Für die Anwendung als Aktor wäre es von großem Nutzen, $\mathrm{Fe}_{75} \mathrm{Pd}_{25}$-Filme in der $\mathrm{L}_{2}$-Struktur, also in einer fct-Struktur mit langreichweitiger Ordnung herzustellen. Diese Ordnung, wie sie zum Beispiel das System Eisen-Platin zeigt, ist bisher experimentell in Eisen-Palladium noch nicht beobachtet worden. Theoretische Berechnungen sagen jedoch für diese Struktur eine maximal erreichbare Dehnung von 6,8 \% voraus, die damit um ein Mehrfaches über der in ungeordneten FePd-Schichten zugänglichen Dehnung liegt [147]. Im Prinzip wäre es denkbar, solche geordnete Strukturen durch abwechselnde Deposition von einer Atomlage Eisen und einer Atomlage $\mathrm{Fe}_{50} \mathrm{Pd}_{50}$ herzustellen, vielversprechender scheint es aber auch hier, die Ordnungseinstellung durch Zulegieren eines dritten Elements zu induzieren. So zeigt sich in Simulationen, dass z.B. in $\mathrm{Fe}_{3}\left(\mathrm{Pd}_{0,95} \mathrm{Ag}_{0,05}\right)$ der Energieunterschied zwischen der geordneten und der ungeordneten Phase leicht reduziert wird [147]. Gelänge es, in einer geeigneten Legierung die Energie der geordneten Phase unter die der ungeordneten abzusenken, wäre es damit unter Umständen möglich, diese Phase durch Tempern bei einer Temperatur einzustellen, bei der einerseits die Entropie noch nicht die ungeordnete Phase stabilisiert, aber andererseits die Legierung auch noch nicht entmischt. 


\section{Anhang A}

\section{Spannungsmessung bei schrägem Einfall der Laserstrahlen}

Eine detaillierte Darstellung und Herleitung der Gleichungen zur Bestimmung der Substratkrümmung findet sich in [77], die allerdings voraussetzt, dass die Laserstrahlen senkrecht (bis auf die durch die Krümmung bewirkte Abweichung) auf das Substrat treffen. Dies lässt sich verallgemeinern, in dem man zunächst die Reflexion eines Laserstrahls an einem allgemeinen Flächenelement betrachtet.

Die Reflexion eines einfallenden Strahls mit dem Richtungsvektor $\vec{e}$ lässt sich so beschreiben, dass die Komponente von $\overrightarrow{e_{p}}$ parallel zur reflektierenden Oberfläche erhalten bleibt, und die Komponente $\overrightarrow{e_{s}}$ senkrecht zu Oberfläche ihr Vorzeichen wechselt. Der Richtungsvektor $\vec{a}$ des ausfallenden Strahls ergibt sich so zu

$$
\vec{a}=\overrightarrow{e_{p}}-\overrightarrow{e_{s}}
$$

Der Vektor $\overrightarrow{e_{s}}$ lässt sich als Skalarprodukt von $\vec{e}$ und dem normierten Normalenvektor der Ebene $\vec{n}$ schreiben. Außerden ergibt die Summe von $\overrightarrow{e_{s}}$ und $\overrightarrow{e_{p}}$ natürlich wieder den ursprünglichen Vektor $\vec{e}$ :

$$
\begin{aligned}
\vec{e} & =\overrightarrow{e_{p}}+\overrightarrow{e_{p}} \\
\overrightarrow{e_{s}} & =(\vec{e} \cdot \vec{n}) \cdot \vec{n} \\
\overrightarrow{e_{p}} & =\vec{e}-\overrightarrow{e_{s}}
\end{aligned}
$$

Somit ergibt sich für den ausfallenden Strahl:

$$
\vec{a}=\overrightarrow{e_{p}}-\overrightarrow{e_{s}}=\vec{e}-\overrightarrow{e_{s}}-\overrightarrow{e_{s}}=\vec{e}-2(\vec{e} \cdot \vec{n}) \cdot \vec{n}
$$




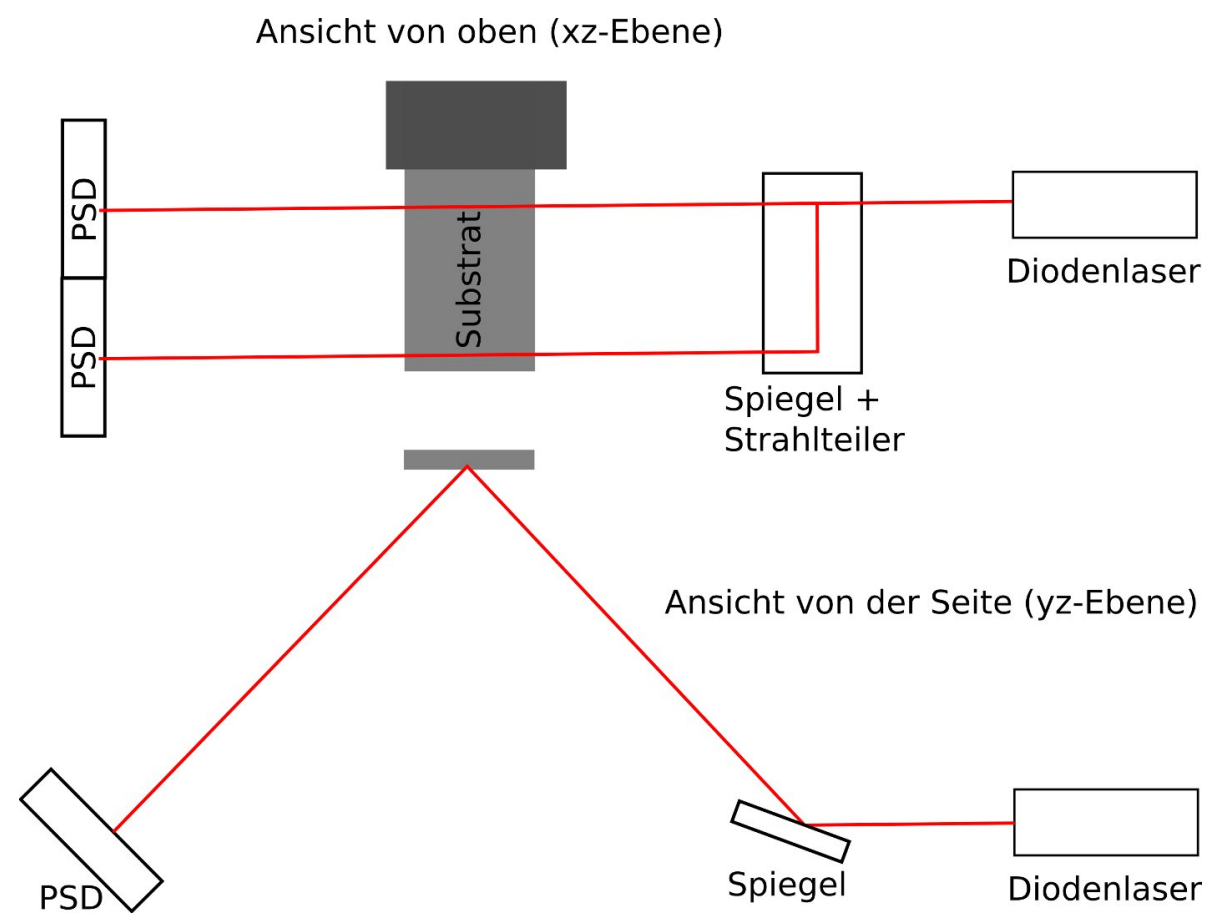

Abbildung A.1: Skizze des Strahlengangs.

Das für die Spannungsmessung verwendete Substrat lässt sich nun nur vor der Deposition durch den Normalenvektor $\overrightarrow{n_{0}}$ beschreiben, während der Deposition hängt der Normalenvektor wegen der Krümmung des Substrats von dem Abstand zur Einspannung ab.

Durch die Wahl eines passenden kartesischens Koordinatensystems, dessen x-Achse parallel zu langen Seite des Substrats und dessen y-Achse parallel zur Normalen des ungekrümmten Substrats steht, lässt sich die folgende Betrachtung nun deutlich vereinfachen: Nimmt man nun an, dass das gekrümmte Substrat einen Kreisbogen bildet, lässt sich der Normalenvektor durch Drehung aus dem ursprünglichen Normalenvektor bestimmen:

$$
\vec{n}=\left(\begin{array}{ccc}
\cos \alpha & \sin \alpha & 0 \\
-\sin \alpha & \cos \alpha & 0 \\
0 & 0 & 1
\end{array}\right) \cdot \overrightarrow{n_{0}}
$$

In dem gewählten Koordinatensystem, dessen y-Achse parallel zur Normalen des ungekrümmten Substrats gewählt würde, vereinfacht sich der allgemeine 
Normalenvektor des gekrümmten Substrats zu:

$$
\vec{n}=\left(\begin{array}{c}
-\sin \alpha \\
-\cos \alpha \\
0
\end{array}\right)
$$

Betrachtet man nun einen allgemeinen einfallenden Lichtstrahl, dessen Richtungsvektor die Koordinaten $x_{e}, y_{e}$ und $z_{e}$ habe, erhält man für den reflektierten Strahl:

$$
\begin{aligned}
\vec{a} & =\vec{e}-2(\vec{e} \cdot \vec{n}) \cdot \vec{n} \\
& =\left(\begin{array}{l}
x_{e} \\
y_{e} \\
z_{e}
\end{array}\right)-2 \cdot\left(-x_{e} \sin \alpha-y_{e} \cos \alpha\right) \cdot\left(\begin{array}{c}
-\sin \alpha \\
-\cos \alpha \\
0
\end{array}\right) \\
& =\left(\begin{array}{c}
x_{e}-2\left(x_{e} \sin ^{2} \alpha+y_{e} \cos \alpha \sin \alpha\right) \\
y_{e}-2\left(x_{e} \sin \alpha \cos \alpha+y_{e} \cos ^{2} \alpha\right) \\
z_{e}
\end{array}\right)
\end{aligned}
$$

Bemerkenswert hierbei ist, dass die $z$-Komponente des Richtungsvektors des einfallenden Strahls keinen Einfluss auf die Ablenkung des Strahls durch das gekrümmte Substrat hat, und daher für die Auswertung nur insofern berücksichtigt werden muss, als dass sie natürlich die Länge des Lichtwegs zwischen Substrat und Detektor mitbestimmt. 
134ANHANG A. SPANNUNGSMESSUNGEN BEI SCHRÄGEM EINFALL 


\section{Literaturverzeichnis}

[1] V. Gehanno, A. Marty, B. Gilles, and Y. Samson. Magnetic domains in epitaxial ordered FePd (001) thin films with perpendicular magnetic anisotropy. Phys. Rev. B, 55(18):12552-12555, 1997.

[2] M. Chen and D. E. Nikles. Synthesis of spherical FePd and CoPt nanoparticles. J. Appl. Phys., 91:8477, 2002.

[3] K. Jessen. Über die Invar-Eigenschaften der Eisen-PalladiumLegierungen. Ann. Phys., 464(5-6):313-315, 1962.

[4] R. R. Duplessis, R. A. Stern, and J. M. MacLaren. Phase stability criteria for cubic and orthorhombic FePt and FePd. J. Appl. Phys., 95:6589, 2004.

[5] W. F. Schlosser. Occurrence of Invar Anomalies in Binary Alloys of Fe with Ni, Pd, Pt, and Mn. J. Appl. Phys., 42:1700, 1971.

[6] R. Hultgren and C. A. Zapffe. Gamma to Alpha Transformation in Iron Alloyed with Palladium. Nature, 142:395-396, 1938.

[7] R. D. James and M. Wuttig. Magnetostriction of martensite. Philos. Mag. A, 77(5):1273-1299, 1998.

[8] Y. Sugimura, T. Cohen-Karni, P. McCluskey, and J. Vlassak. Fabrication and characterization of Fe-Pd ferromagnetic shape-memory thin films. Materials and Devices for Smart Systems, edited by Y. Furuya, E. Quandt, Q. Zhang, K. Inoue, and M. Shahinpoor (Mater. Res. Soc. Symp. Proc. 785, Pittsburgh, PA, 2003) D, 7, 2003.

[9] J. Buschbeck, L. Schultz, and S. Fähler. Epitaxial Fe-Pd Magnetic Shape Memory Films-Issues for Preparation and Applications. Proc. Actuator 2008, 11th Int. Conf. on New Actuators, Bremen, Germany, page $750,2008$. 
[10] O. Söderberg, A. Sozinov, and V. K. Lindroos. Giant magnetostrictive materials. Handbook of Magnetic Materials, 16:1-39, 2006.

[11] C. Bechtold, I. Teliban, C. Thede, S. Chemnitz, and E. Quandt. Noncontact strain measurements based on inverse magnetostriction. Sens. Actuators A, 2010.

[12] E. C. Bain and N. Y. Dunkirk. The nature of martensite. Trans. AIME, 70:25, 1924.

[13] J. Buschbeck, I. Opahle, M. Richter, U. K. Rössler, P. Klaer, M. Kallmayer, H. J. Elmers, G. Jakob, L. Schultz, and S. Fähler. Full tunability of strain along the fcc-bcc bain path in epitaxial films and consequences for magnetic properties. Phys. Rev. Lett., 103(21):216101, 2009.

[14] M. E. Gruner, W. A. Adeagbo, A. T. Zayak, A. Hucht, and P. Entel. Lattice dynamics and structural stability of ordered $\mathrm{Fe}_{3} \mathrm{Ni}, \mathrm{Fe}_{3} \mathrm{Pd}$ and $\mathrm{Fe}_{3} \mathrm{Pt}$ alloys using density functional theory. Phys. Rev. B, 81(6):64109, 2010 .

[15] M. E. Gruner. unpublished. 2010.

[16] T. Nakayama, M. Kikuchi, and K. Fukamichi. Young's Modulus and the $\Delta \mathrm{E}$ Effect of Fe-Pd Invar Alloys. J. Phys. F, 10(4):715-719, 1980.

[17] H. M. Matsui, H. Yamada, and K. Adachi. A New Low Temperature Phase (fct) of Fe-Pd Invar. J. Phys. Soc. Jpn., 48(6):2161-2162, 1980.

[18] M. Foos, C. Frantz, and M. Gantois. Structural changes in invar iron-palladium alloys before the fcc-bct martensitic transformation. $J$. Phys., 43:C4-389 - C4-394, 1982.

[19] M. Matsui and K. Adachi. Magnetostriction of Fe-Pd Invar. J. Magn. Magn. Mater., 31-34:115-116, (1983).

[20] M. Matsui, T. Shimizu, and K. Adachi. Invar anomalies of Fe-Pd alloys. Physica $B+C, 119(1-2): 84-89,1983$.

[21] M. Sugiyama, R. Oshima, and F. E. Fujita. Martensitic transformation in the iron-palladium alloy system. Trans. Jpn. Inst. Met, 25(9), 1984.

[22] M. Sugiyama, R. Oshima, and F. E. Fujita. Mechanism of FCC-FCT Thermoelastic Martensite Transformation in Fe-Pd Alloys. Trans. Jpn. Inst. Met., 27(10):719-730, 1986. 
[23] Y. Ohta. Local stresses and elastic softening of Fe-Pd invar alloys. Physica B, 161(1-3):60-62, 1989.

[24] K. Ullakko, J. K. Huang, C. Kantner, R. C. O'Handley, and V. V. Kokorin. Large magnetic-field-induced strains in $\mathrm{Ni}_{2} \mathrm{MnGa}$ single crystals. Appl. Phys. Lett., 69(13):1966, 1996.

[25] B. Spasova, M. C. Wurz, and H. H. Gatzen. Manufacturing technology for the fabrication a discrete actuator using a magnetic shape memory alloy. Eur. Phys. J. - Special Topics, 158(1):243-247, 2008.

[26] M. Chmielus, X. X. Zhang, C. Witherspoon, D. C. Dunand, and P. M üllner. Giant magnetic-field-induced strains in polycrystalline $\mathrm{Ni}-\mathrm{Mn}-$ Ga foams. Nature Mat., 2009.

[27] H. Kato, Y. Liang, and M. Taya. Stress-induced FCC/FCT phase transformation in Fe-Pd alloy. Scr. Mater., 46(6):471-475, 2002.

[28] 1\%. IFF-Ferienkurs: Dünne Schichten und Schichtsysteme. Forschungszentrum Jülich GmbH, 1986.

[29] A. Rajamani, B. W. Sheldon, E. Chason, and A. F. Bower. Intrinsic tensile stress and grain boundary formation during Volmer-Weber film growth. Appl. Phys. Lett., 81:1204, 2002.

[30] T. B. Massalski. Binary Alloy Phase Diagrams. American Society for Metals, 1986.

[31] P. Haasen. Physikalische Metallkunde. Springer-Verlag, 3 edition, 1994.

[32] I. Kock, S. Hamann, T. Edler, S. G. Mayr, and A. Ludwig. Development and characterization of $\mathrm{Fe}_{70} \mathrm{Pd}_{30}$ ferromagnetic shape memory splats. Intermetallics, 18(5):877 - 882, 2010.

[33] I. Kock, T. Edler, and S. G. Mayr. Growth behavior and intrinsic properties of vapor-deposited iron palladium thin films. J. Appl. Phys., 103:046108, 2008.

[34] L. Kühnemund, T. Edler, I. Kock, M. Seibt, and S. G. Mayr. Epitaxial growth and stress relaxation of vapor-deposited Fe-Pd magnetic shape memory films. N. J. Phys., 11:113054, 2009.

[35] J. Buschbeck, I. Lindemann, L. Schultz, and S. Fähler. Growth, structure, and texture of epitaxial $\mathrm{Fe}_{100-x} \mathrm{Pd}_{x}$ films deposited on $\mathrm{MgO}(100)$ at room temperature: An X-ray diffraction study. Phys. Rev. B, 76(20):205421, 2007. 
[36] T. Kakeshita and T. Fukuda. Giant magnetostriction in $\mathrm{Fe}_{3} \mathrm{Pt}$ and FePd ferromagnetic shape-memory alloys. Materials Science Forum(Switzerland), 394:531-536, 2001.

[37] T. Kakeshita, T. Fukuda, T. Terai, T. Takeuchi, and K. Kishio. Martensitic transformation in Fe-based shape memory alloys under magnetic field. J. Phys. IV France, 112:93, 2003.

[38] M. R. Hestenes and E. Stiefel. Methods of conjugate gradients for solving linear systems. J. Res. Nat. Bur. Stand., 1952.

[39] J. Cui, T. W. Shield, and R. D. James. Phase transformation and magnetic anisotropy of an iron-palladium ferromagnetic shape-memory alloy. Acta Mat., 52(1):35-47, 2004.

[40] P. Wollants, J. R. Roos, and L. Delaey. Thermally-and stress-induced thermoelastic martensitic transformations in the reference frame of equilibrium thermodynamics. Prog. Mater. Sci., 37(3):227-288, 1993.

[41] H. Harms. Mechanische Spannungen und Mikrostruktur dünner TiNiund $\mathrm{Ti}_{50} \mathrm{Ni}_{50-x} \mathrm{Cu}_{x}$-Formgedächtnisschichten. Dissertation, GeorgAugust-Universität Göttingen, 2003.

[42] C. E. Guillaume. Recherches sur les aciers au nickel. Dilatations aux températures élevées; résistance électrique. CR Acad. Sci, 125(235):18, 1897.

[43] P. Entel, E. Hoffmann, P. Mohn, K. Schwarz, and V. L. Moruzzi. Firstprinciples calculations of the instability leading to the Invar effect. Phys. Rev. B, 47(14):8706-8720, 1993.

[44] R. J. Weiss. The origin of the'Invar'effect, 1963.

[45] E. F. Wassermann. Invar: Moment-volume instabilities in transition metals and alloys. Ferromagnetic materials, 5:237, 1990.

[46] M. Acet, H. Zähres, E. F. Wassermann, and W. Pepperhoff. Hightemperature moment-volume instability and anti-Invar of $\gamma$-Fe. Phys. Rev. B, 49(9):6012-6017, 1994.

[47] M. van Schilfgaarde, I. A. Abrikosov, and B. Johansson. Origin of the Invar effect in iron-nickel alloys. Nature, 400(6739):46-49, 1999.

[48] A. Nagasawa. Nature of martensitic phase transition in $\beta$ phase alloys. J. Phys. Soc. Jpn., 40(4):1021-1026, 1976. 
[49] S. Muto, R. Oshima, and F. E. Fujita. Elastic softening and elastic strain energy consideration in the fcc-fct transformation of $\mathrm{Fe}-\mathrm{Pd}$ alloys. Acta Metall. Mat., 38(4):685-694, 1990.

[50] R. Oshima, S. Muto, and F. E. Fujita. Initiation of FCC-FCT Thermoelastic Martensite Transformation From Premartensitic State of Fe-30 at.\% Pd Alloys. Mater. Trans., JIM, 33(3):197-202, 1992.

[51] I. Opahle, K. Koepernik, U. Nitzsche, and M. Richter. Jahn-Teller-like origin of the tetragonal distortion in disordered $\mathrm{Fe}-\mathrm{Pd}$ magnetic shape memory alloys. Appl. Phys. Lett., 94:072508, 2009.

[52] J. Kuang, M. Kontani, M. Matsui, and K. Adachi. Electronic, phonon and magnon specific heats of Fe-Pd alloys. Physica B+ C, 149(13):209-216, 1988.

[53] R. C. O'Handley. Modern magnetic materials: principles and applications. Wiley-Interscience New York, 1999.

[54] E. Bauer. Phänomenologische Theorie der Kristallabscheidung an Oberflächen. Z. Kristallogr., 110(372):1820, 1958.

[55] J. A. Thornton. High rate thick film growth. Annu. Rev. Mater. Sci., 7(1):239-260, 1977.

[56] J. A. Thornton and D. W. Hoffmann. Stress-related effects in thin films. Thin Solid Films, 171(1):5-31, 1989.

[57] D. Walton, T. N. Rhodin, and R. W. Rollins. Nucleation of silver on sodium chloride. J. Chem. Phys., 38:2698, 1963.

[58] B. W. Sloope and C. O. Tiller. Formation Conditions and Structure of Ge Films Deposited on Polished (111) CaF Substrates in an UltrahighVacuum System. J. Appl. Phys., 36:3174, 1965.

[59] J. F. Ziegler, J. P. Biersack, and M. D. Ziegler. SRIM-The stopping and range of ions in matter. SRIM Co., 2008.

[60] J. F. Ziegler, J. P. Biersack, and U. Littmark. The stopping and range of ions in matter. Pergamon, New York, 1985.

[61] J. R. Tesmer, M. Nastasi, J. C. Barbour, C. J. Maggiore, and J. W. Mayer. Handbook of modern ion beam materials analysis. Materials Research Society Pittsburgh, PA, 1995. 
[62] R. S. Averback and T. Diaz de la Rubia. Displacement damage in irradiated metals and semiconductors. Solid state physics(New York. 1955), 51:281-402, 1997.

[63] K. Nordlund, M. Ghaly, RS Averback, M. Caturla, T. Diaz de La Rubia, and J. Tarus. Defect production in collision cascades in elemental semiconductors and fcc metals. Phys. Rev. B, 57(13):7556-7570, 1998.

[64] K. Tsutsui and S. Furukawa. Ion beam mixing of Pt/GaAs and formation of ohmic contacts. J. Appl. Phys., 56(2):560-562, 1984.

[65] J. Nord, K. Nordlund, and J. Keinonen. Amorphization mechanism and defect structures in ion-beam-amorphized Si, Ge, and GaAs. Phys. Rev. $B, 65(16): 165329,2002$.

[66] J. K. Hirvonen. Ion beam assisted thin film deposition. Materials Science Reports, 6(6):215-274, 1991.

[67] M. Marinov. Effect of ion bombardment on the initial stages of thin film growth. Thin Solid Films, 46(3):267-274, 1977.

[68] G. E. Lane and J. C. Anderson. The nucleation and initial growth of gold films deposited onto sodium chloride by ion-beam sputtering. Thin Solid Films, 26(1):5-23, 1975.

[69] L. Pranevicius. Structure and properties of deposits grown by ionbeam-activated vacuum deposition techniques. Thin Solid Films, 63(1):77-85, 1979.

[70] E. Chason, P. Bedrossian, K. M. Horn, J. Y. Tsao, and S. T. Picraux. Ion beam enhanced epitaxial growth of Ge (001). Appl. Phys. Lett., $57: 1793,1990$.

[71] S. Esch, M. Breeman, M. Morgenstern, T. Michely, and G. Comsa. Nucleation and morphology of homoepitaxial Pt (111)-films grown with ion beam assisted deposition. Surf. Sci., 365(2):187-204, 1996.

[72] S. G. Mayr. Wachstum amorpher Schichten: Vergleich von Experiment und Simulation im Bereich Oberflächenrauigkeit und mechanische Spannungen. Dissertation, Universität Göttingen, 2000.

[73] C. Streng. Wachstumsanalyse amorpher dicker Schichten und Schichtsysteme. Dissertation, Universität Göttingen, 2004. 
[74] S. Fähler and H. U. Krebs. Calculations and experiments of material removal and kinetic energy during pulsed laser ablation of metals. Appl. Surf. Sci., 76(1):61-65, 1996.

[75] H. U. Krebs, M. Störmer, S. Fähler, O. Bremert, M. Hamp, A. Pundt, H. Teichler, W. Blum, and T. H. Metzger. Structural properties of laser deposited metallic alloys and multilayers. Appl. Surf. Sci., 109:563-569, 1997.

[76] S. Thienhaus. Mikrostruktur und Phasenbildung hochorientierter TiNiCu- und NiMnAl-Formgedächtnisschichten, hergestellt mittels Molekularstrahl-Epitaxie. Dissertation, Universität Bonn, 2005.

[77] M. Bicker. Messung von mechanischen Spannungen in dünnen Schichten mit einer Zwei-Strahl-Lichtzeigerdetektion. Diplomarbeit, Universität Göttingen, 1996.

[78] G. G. Stoney. The tension of metallic films deposited by electrolysis. Proc. R. Soc., A 82:172-175, 1909.

[79] Gemeinfrei / Public Domain. Ethylendiamintetraessigsäure - Wikipedia, Die freie Enzyklopädie, 2010. [Online; Stand 25. Januar 2010].

[80] M. Thomas, O. Heczko, J. Buschbeck, Y. W. Lai, J. McCord, S. Kaufmann, L. Schultz, and S. Fähler. Stray-Field-Induced Actuation of Free-Standing Magnetic Shape-Memory Films. Adv. Mat., 21(36):3708-3711, 2009.

[81] A. Backen, S.R. Yeduru, M. Kohl, S. Baunack, A. Diestel, B. Holzapfel, L. Schultz, and S. Fähler. Comparing properties of substrateconstrained and freestanding epitaxial Ni-Mn-Ga films. Acta Mat., 58(9):3415-3421, 2010.

[82] F. Khelfaoui, M. Kohl, J. Buschbeck, O. Heczko, S. Fähler, and L. Schultz. A fabrication technology for epitaxial Ni-Mn-Ga microactuators. Eur. Phys. J. Special Topics, 158(1):167-172, 2008.

[83] L. G. Parratt. Surface studies of solids by total reflection of x-rays. Phys. Rev., 95(2):359-369, Jul 1954.

[84] W. Kraus and G. Nolze. POWDER CELL-a program for the representation and manipulation of crystal structures and calculation of the resulting X-ray powder patterns. J. Appl. Cryst., 29(3):301-303, 1996. 
[85] P. Villars. Pearson's Handbook: Crystallographic Data for Intermetallic Phases. ASM International (OH), 1991.

[86] M. Birkholz. Thin Film Analysis by X-Ray Scattering. Wiley-VCH, 2006 .

[87] W. C. Oliver and G. M. Pharr. Improved technique for determining hardness and elastic modulus using load and displacement sensing indentation experiments. J .Mater. Res., 7(6):1564-1583, 1992.

[88] L. Doolittle and M. Thompson. RUMP RBS Simulation Program. Computer Graphics Service, 1993.

[89] MPMS Hardware Reference Manual. Quantum Design.

[90] M. Neudecker and S. G. Mayr. Dynamics of shear localization and stress relaxation in amorphous $\mathrm{Cu}_{50} \mathrm{Ti}_{50}$. Acta Mat., 2008.

[91] C. W. Gear. The automatic integration of ordinary differential equations. Commun. ACM, 14(3):179, 1971.

[92] M. P. Allen and D. J. Tildesley. Computer simulation of liquids. Oxford University Press, USA, 1990.

[93] D. C. Rapaport. The art of molecular dynamics simulation. Cambridge Univ. Press, 2004

[94] F. H. Stillinger and T. A. Weber. Computer simulation of local order in condensed phases of silicon. Phys. Rev. B, 31(8):5262-5271, 1985.

[95] M. Müller, P. Erhart, and K. Albe. Thermodynamics of $\mathrm{L1}_{0}$ ordering in FePt nanoparticles studied by Monte Carlo simulations based on an analytic bond-order potential. Phys. Rev. B, 76(15):155412, 2007.

[96] D. W. Brenner. Empirical potential for hydrocarbons for use in simulating the chemical vapor deposition of diamond films. Phys. Rev. B, 42(15):9458-9471, Nov 1990.

[97] K. Albe, K. Nordlund, and R. S. Averback. Modeling the metal-semiconductor interaction: Analytical bond-order potential for platinum-carbon. Phys. Rev. B, 65(19):195124, 2002.

[98] M. S. Daw and M. I. Baskes. Embedded-atom method: Derivation and application to impurities, surfaces, and other defects in metals. Phys. Rev. B, 29(12):6443-6453, 1984. 
[99] H. J. C. Berendsen, J. P. M. Postma, W. F. van Gunsteren, A. DiNola, and J. R. Haak. Molecular dynamics with coupling to an external bath. J. Chem. Phys., 81:3684, 1984.

[100] J. Li. AtomEye: an efficient atomistic configuration viewer. Modell. Simul. Mater. Sci. Eng., 11(2):173-177, 2003.

[101] C. L. Kelchner, S. J. Plimpton, and J. C. Hamilton. Dislocation nucleation and defect structure during surface indentation. Phys. Rev. B, 58(17):11085-11088, Nov 1998.

[102] I. Kock. Wachstum und mechanische Spannungen an dünnen Schichten der magnetischen Formgedächtnislegierung Eisen-Palladium. Diplomarbeit, Universität Göttingen, 2007.

[103] T. Edler, J. Buschbeck, C. Mickel, S. Fähler, and S. G. Mayr. Mechanisms of stress generation and relaxation during pulsed laser deposition of epitaxial Fe-Pd magnetic shape memory alloy films on $\mathrm{MgO}$. N. J. Phys., 10:063007, 2008.

[104] F. Cardarelli. Materials handbook. Springer London etc., 2000.

[105] L. Kühnemund. Epitaktisches Wachstum von dünnen Schichten der magnetischen Formgedächtnislegierung FePd auf MgO. Diplomarbeit, Universität Göttingen, 2009.

[106] J. J. Gilvarry. The Lindemann and Grüneisen laws. Phys. Rev., 102(2):308-316, 1956.

[107] G. Hausch. Elastic Constants of Fe-Pt Alloys: I. Single Crystalline Elastic Constants of $\mathrm{Fe}_{72} \mathrm{Pt}_{28}$. J. Phys. Soc. Jpn., 37(3), 1974.

[108] S. Thienhaus, C. Zamponi, H. Rumpf, J. Hattrick-Simpers, I. Takeuchi, and A. Ludwig. High-Throughput Characterization of Shape Memory Thin Films Using Automated Temperature-Dependent Resistance Measurements. In Mater. Res. Soc. Symp. Proc., volume 894, page 197, 2006.

[109] A. Ludwig, R. Zarnetta, S. Hamann, A. Savan, and S. Thienhaus. Development of multifunctional thin films using high-throughput experimentation methods. Z. Metallkd. / Int. J. Mater. Res., 99(10), 2008.

[110] D. E. Gray. American institute of physics handbook. Am. J. Phys., 32:389, 1972. 
[111] T. Edler, S. Hamann, A. Ludwig, and S. G. Mayr. Reversible fcc $\leftrightarrow$ bcc transformation in freestanding epitaxially grown Fe-Pd ferromagnetic shape memory films. Submitted to Appl. Phys. Lett., 2010.

[112] J. C. Bertolini, J. L. Rousset, P. Miegge, J. Massardier, B. Tardy, Y. Samson, B. C. Khanra, and C. Creemers. Surface segregation study of a dilute $\mathrm{Pd}_{1} \mathrm{Fe}_{99}$ alloy by LEIS and XPS. Surf. Sci., 281(1-2):102 110, 1993.

[113] M. Moseler, P. Gumbsch, C. Casiraghi, A.C. Ferrari, and J. Robertson. The ultrasmoothness of diamond-like carbon surfaces. Science, 309(5740):1545, 2005.

[114] S. Vauth. Glättungsmechanismen beim Ionenbeschuss rauer amorpher Oberflächen. Dissertation, Universität Göttingen, 2007.

[115] B. D. Cullity. Elements of X-Ray Diffraction. Addison-Wesley Reading, MA, (1978).

[116] C. Duriez, C. Chapon, C. R. Henry, and J. M. Rickard. Structural characterization of MgO (100) surfaces. Surf. Sci., 230(1-3):123-136, 1990 .

[117] M. Störmer, K. Sturm, S. Fähler, M. Weisheit, J. Winkler, S. Kahl, P. Kesten, A. Pundt, M. Seibt, S. Senz, and H. U. Krebs. Study of laser-deposited metallic thin films by a combination of high-resolution ex situ and time-resolved in situ experiments. Appl. Phys. A: Mat. Science $\&$ Processing, 69(7):455-457, 1999.

[118] H. U. Krebs, O. Bremert, Y. Luo, S. Fähler, and M. Störmer. Structure of laser-deposited metallic alloys and multilayers. Thin Solid Films, 275(1-2):18-21, 1996.

[119] H. Sakai, S. Keitoku, and H. Ezumi. Contribution of High-Velocity Particles to Stress of Thin Film Produced by Laser Ablation. Jpn. J. Appl. Phys, 36:L409-L411, 1997.

[120] S. G. Mayr, M. Moske, K. Samwer, M. E. Taylor, and H. A. Atwater. The role of particle energy and pulsed particle flux in physical vapor deposition and pulsed-laser deposition. Appl. Phys. Lett., 75:4091, 1999 .

[121] S. G. Mayr and R. S. Averback. Effect of ion bombardment on stress in thin metal films. Phys. Rev. B, 68(21):214105, 2003. 
[122] N. Durand, K. F. Badawi, and P. Goudeau. Residual stresses and microstructure in tungsten thin films analyzed by x-ray diffractionevolution under ion irradiation. J. Appl. Phys., 80:5021, 1996.

[123] Q. Wang, H. Ogiso, S. Nakano, J. Akedo, and H. Ishikawa. Martensitic transformation and the stress induced by $3 \mathrm{MeV}$ ion implantation in an austenite stainless steel sheet. Nuclear Inst. Meth. B, 206:118-122, 2003 .

[124] Q. Wang, H. Ishikawa, S. Nakano, H. Ogiso, and J. Akedo. Curvaturebased residual stress measurement for ion-implanted stainless-steel sheet. Vacuum, 75(3):225-229, 2004.

[125] D. Halley, Y. Samson, A. Marty, P. Bayle-Guillemaud, C. Beigné, B. Gilles, and J. E. Mazille. Anomaly of strain relaxation in thin ordered FePd layers. Phys. Rev. B, 65(20):205408, 2002.

[126] D. Halley, A. Marty, P. Bayle-Guillemaud, B. Gilles, J. P. Attane, and Y. Samson. Chemical order and selection of the mechanism for strain relaxation in epitaxial FePd $(\mathrm{Pt})$ thin layers. Phys. Rev. B, 70(17):174438, 2004.

[127] O. V. Angelsky, S. G. Hanson, and P. P. Maksimyak. Use of optical correlation techniques for characterizing scattering objects and media. SPIE Press, 1999.

[128] B. G. Orr, D. Kessler, C. W. Snyder, and L. Sander. A model for strain-induced roughening and coherent island growth. Europhys. Lett., 19:33-38, 1992.

[129] M. Matsui, T. Shimizu, H. Yamada, and K. Adachi. Magnetic Properties and thermal expansion of Fe-Pd Invar Alloys. J. Magn. Magn. Mater., 15-18:1201-1202, 1980.

[130] H. Watanabe, N. Yamada, and M. Okaji. Linear thermal expansion coefficient of silicon from 293 to 1000 K. I. J. Thermophys., 25(1):221$236,2004$.

[131] I. Kock. Untersuchung der Phasenstabilität und des martensitischen Phasenübergangs in Eisen-Palladiumlegierungen. Dissertation, Universität Göttingen, 2010, unpublished. 
[132] C. Bechtold, J. Buschbeck, A. Lotnyk, B. Erkartal, S. Hamann, C. Zamponi, L. Schultz, A. Ludwig, S. Kienle, S. Fähler, and E. Quandt. Artificial Single Variant Martensite in Freestanding $\mathrm{Fe}_{70} \mathrm{Pd}_{30}$ Films Obtained by Coherent Epitaxial Growth. Adv. Mat., accepted, 2010.

[133] J. Koeda, Y. Nakamura, T. Fukuda, T. Kakeshita, T. Takeuchi, and K. Kishio. Giant Magnetostriction in Fe-Pd Alloy Single Crystal Exhibiting Martensitic Transformation. Trans. Mater. Res. Soc. Jpn., 26(1):215-218, 2001.

[134] T. Kakeshita and T. Fukuda. Magnetic field-control of microstructure and function of materials exhibiting solid-solid phase transformation. Sci. Technol. Adv. Mater., 7(4):350-355, 2006.

[135] R. Oshima, K. Kosuga, M. Sugiyama, and F. E. Fujita. Phase Transition in Fe-Pd Alloys. Sci. Rep. Res. Inst. Tohoku Univ. A, 29:67-72, 1981.

[136] R. Oshima and M. Sugiyama. Martensite Transformations in Fe-Pd Alloys. Le Journal de Physique Colloques, 43(C4):4-4, 1982.

[137] C. Engin and H. M. Urbassek. Molecular-dynamics investigation of the fcc $\rightarrow$ bcc phase transformation in Fe. Comput. Mater. Sci., 41(3):297304, 2008.

[138] K. Seki, H. Kura, T. Sato, and T. Taniyama. Size dependence of martensite transformation temperature in ferromagnetic shape memory alloy FePd. J. Appl. Phys., 103:063910, 2008.

[139] K. Sumiyama, H. Fujimori, N. Kataoka, J. Y. Kim, and S. L. Zhang. Magnetic Properties of Nonequilibrium Fe-Pd Alloys Produced by DC Triode Sputtering. Jap. J. Appl. Phys., 29(7):1261-1265, 1990.

[140] G. S. Yang, R. Jonasson, S. N. Baek, K. Murata, S. Inoue, K. Koterazawa, S.J. Jeong, K. Mizuuchi, and K. Inoue. Phase Transformations of Ferromagnetic Fe-Pd-Pt-Based Shape Memory Alloys. In Mater. Res. Soc. Symp. Proc., volume 785, pages 475-480, 2004.

[141] T. Wada, T. Tagawa, and M. Taya. Martensitic transformation in Pdrich Fe-Pd-Pt alloy. Scripta mat., 48(2):207-211, 2003.

[142] D. Vokoun, Y. W. Wang, T. Goryczka, and C. T. Hu. Magnetostrictive and shape memory properties of $\mathrm{Fe}-\mathrm{Pd}$ alloys with $\mathrm{Co}$ and $\mathrm{Pt}$ additions. Smart Mater. Struct., 14:S261-S265, 2005. 
[143] K. Tsuchiya, T. Nojiri, H. Ohtsuka, and M. Umemoto. Effect of Co and $\mathrm{Ni}$ on martensitic transformation and magnetic properties in $\mathrm{Fe}-\mathrm{Pd}$ ferromagnetic shape memory alloys: Structural and functional control of materials through solid-solid phase transformations in high magnetic field. Mater. Trans. JIM, 44(12):2499-2502, 2003.

[144] S. Hamann, M. E. Gruner, S. Irsen, J. Buschbeck, C. Bechtold, I. Kock, S. G. Mayr, A. Savan, S. Thienhaus, E. Quandt, S. Fähler, P. Entel, and A. Ludwig. The ferromagnetic shape memory system Fe-Pd-Cu. Submitted to Acta Mat., 2010.

[145] V. Sánchez-Alarcos, V. Recarte, J. I. Pérez-Landazábal, M. A. González, and J. A. Rodríguez-Velamazán. Effect of Mn addition on the structural and magnetic properties of Fe-Pd ferromagnetic shape memory alloys. Acta Mat., 57(14):4224-4232, 2009.

[146] R. A. Stern, S. D. Willoughby, A. Ramirez, J. M. MacLaren, J. Cui, Q. Pan, and R. D. James. Electronic and structural properties of FePdPt ferromagnetic shape memory alloys. J. Appl. Phys., 91:7818, 2002.

[147] R. A. Stern, S. D. Willoughby, J. M. MacLaren, J. Cui, Q. Pan, and R. D. James. $\mathrm{Fe}_{3} \mathrm{Pd}$ ferromagnetic shape memory alloys. J. Appl. Phys., 93(10):8644-8646, 2003. 


\section{Danksagung}

An dieser Stelle möchte ich mich bei allen denjenigen sehr herzlich bedanken, die auf die eine oder andere Weise zum Entstehen dieser Arbeit beigetragen haben.

Herrn Prof. Stefan Mayr danke ich für die Betreuung meiner Arbeit. Er hat es mir ermöglicht dieses spannende und hochaktuelle Forschungsgebiet $\mathrm{zu}$ bearbeiten, und hat mich sowohl von Göttingen, als auch später von Leipzig aus, auf vielfältige Weise unterstützt und hatte immer ein offenes Ohr bei experimentellen oder sonstigen Problemen.

Herrn Prof. Reiner Kirchheim danke ich für seine Bereitschaft, das Koreferat zu übernehmen und Mitglied im Betreuungsausschuss zu werden.

Für die finanzielle Unterstützung bedanke ich mich bei der Deutschen Forschungsgemeinschaft für die Förderung im Rahmen des Schwerpunktprojektes 1239 „Änderung von Mikrostruktur und Form fester Werkstoffe durch äußere Magnetfelder “, Teilprojekt C4.

Ganz besonders möchte ich mich auch bei Herrn Prof. Konrad Samwer für seine Unterstützung während meiner gesamten Zeit im I. Physikalischen Institut bedanken.

Ich danke den jetzigen und ehemaligen Mitgliedern der MSM-Gruppe Iris Kock, Guido Mahnke, und Lisa Kühnemund für die gute Zusammenarbeit, die vielen Diskussionen und ihre tatkräftige Unterstützung bei diversen Umbauten und Reparaturen an den UHV-Anlagen. Auch bei allen anderen (ehemaligen) Mitarbeitern der Arbeitsgruppe Mayr bedanke ich mich für die experimentelle Unterstützung und die Einführung in die diversen Anlagen und Messmethoden. Sowohl bei den schon genannten als auch bei allen anderen Mitgliedern des I. Physikalischen Instituts bedanke ich mich sehr herzlich für das sehr angenehme Arbeitsklima und ihre Hilfsbereitschaft. 
Viele Messungen in dieser Arbeit wurden in Kooperationen mit anderen Wissenschaftlern durchgeführt, ohne deren gemeinsame Diskussion manche Erkenntnisse nicht zu erreichen gewesen wären. Ich danke ganz besonders Sven Hamann und Prof. Alfred Ludwig für die vielen Röntgen- EDX- und Widerstandsmessungen, während und außerhalb meines Aufenthalts an der Universität Bochum, Jörg Buschbeck und Sebastian Fähler für die gemeinsamen Spannungsmessungen bei der gepulsten Laserdeposition während meines Aufenthalts in Dresden, Prof. Michel Seibt und Volker Radisch für die TEM-Untersuchungen und die Präparation der Lamellen, Inga Knorr und Prof. Cynthia Volkert für die Nanoindenter-Messungen an einkristallinen Schichten, Helmut Klein und Heidrun Sowa für die Röntgenmessungen mit dem Flächendetektor, Kuhn Zhang und Prof. Hans Hofsäß für die RBSMessungen, Andre Pape, Marc Wurz und Prof. Hans-Heinrich Gatzen für die Nanoindenter-Messungen an polykristallinen Schichten, sowie See Wee Chee und Prof. Robert Averback für die Bestrahlung der polykristallinen Filme.

Mein ganz besonderer Dank gilt Carsten Mahn für seine moralische und technische Unterstützung beim Reparieren und Umbauen der UHV-Anlagen und bei allen elektronischen Problemen, Uta Filippich für ihre Hilfe im Umgang mit dem REM und das Aufdampfen von Schichten, und Antje Krüger für das Anfertigen von REM-Aufnahmen und EDX-Analysen, Kathrin Gerke für die Wartung der Röntgenanlagen, sowie der Zentralwerkstatt und der feinmechanischen Werkstatt des I. Physikalischen Instituts für ihre Unterstützung bei Reparaturen und Neubauten.

Für das Korrekturlesen dieser Arbeit möchte ich mich bei Nicole Edler, Britta Fuchs, Iris Kock und Dennis Bedorf bedanken.

Der größte Dank gebührt meiner Frau Nicole, für ihre unermüdliche Unterstützung und ihr Verständnis, durch alle Höhen und Tiefen dieser Arbeit hinweg. Ohne dich wäre diese Arbeit nie zustande gekommen. 


\section{Publikationen}

2007

2008

2009

2010

T. Edler und S.G. Mayr:

Mechanisms of stress generation during bombardment of Ge with keV ions: experiments and molecular dynamics simulations

New Journal of Physics 9325 (2007)

I. Kock, T. Edler und S.G. Mayr:

Growth behavior and intrinsic properties of vapor-deposited iron palladium thin films

Journal of Applied Physics 103046108 (2008)

T. Edler, J. Buschbeck, C. Mickel, S. Fähler und S.G. Mayr: Mechanisms of stress generation and relaxation during pulsed laser deposition of epitaxial $F e-P d$ magnetic shape memory alloy films on $\mathrm{MgO}$

New Journal of Physics 10063007 (2008)

L. Kühnemund, T. Edler, I. Kock, M. Seibt und S.G. Mayr: Epitaxial growth and stress relaxation of vapor-deposited FePd magnetic shape memory films

New Journal of Physics 11113054 (2009)

I. Kock, S. Hamann, H. Brunken, T. Edler, S.G. Mayr und A. Ludwig:

Development and characterization of Fe'70Pd30 ferromagnetic shape memory splats

Intermetallics 18 (5) 877 (2010)

T. Edler, S. Hamann, A. Ludwig und S.G. Mayr:

Reversible $f c c \leftrightarrow$ bcc transformation in freestanding epitaxially grown $\mathrm{Fe}-\mathrm{Pd}$ ferromagnetic shape memory films

Applied Physics Letters, submitted (2010) 



\section{Lebenslauf}

\section{Persönliche Daten}

Tobias Edler

Geburtsdatum: 26. Mai 1981

Geburtsort: Steinheim a. A.

Familienstand: verheiratet

Staatsangehörigkeit: deutsch

\section{Schulbildung}

1987-1991

Phillip-Friedrich-Hiller-Grundschule, Steinheim a. A.

$1991-2000$

Max-Planck-Gymnasium Heidenheim

21.06 .2000

Allgemeine Hochschulreife

\section{Zivildienst}

2000-2001

Arbeiterwohlfahrt Heidenheim

\section{Studium}

10/2001-11/2006 Studiengang „Diplomphysik“an der Georg-August-Universität Göttingen

04/2005-11/2006 Diplomarbeit im Bereich Festkörperphysik, Betreuer Prof. S. Mayr, Thema „Mechanische Spannungen beim Beschuss von Germanium mit niederenergetischen Edelgasionen: Simulationen und Experimente "

10.11.2006 Diplom in Physik, (Note „sehr gut“)

seit 03/2007 Promotionsstudium im Fach Physik an der Georg-AugustUniversität Göttingen

seit 12/2006 Wissenschaftlicher Mitarbeiter am I. Physikalischen Institut der Georg-August-Universität Göttingen und anfertigung der vorliegenden Dissertation

19. Mai 2010 
